\title{
UNDERSTANDING PIKFYVE CONTROL OF LYSOSOME DYNAMICS
}

By

\author{
Golam Tanjib Saffi
}

BSc - Honours Science and Business/Biotechnology specialization, University of Waterloo, 2012

MSc - Pharmaceutical Science, University of Waterloo, 2014

A dissertation submitted to Ryerson University

in partial fulfillment of the requirements

for the degree of

Doctor of Philosophy

in the program of

Molecular Science

Toronto, Ontario, Canada, 2019

(C) Golam T. Saffi, 2019 


\section{Author's declaration}

I hereby declare that I am the sole author of this dissertation. This is a true copy of the dissertation, including any required final revisions, as accepted by my examiners.

I authorize Ryerson University to lend this dissertation to other institutions or individuals for the purpose of scholarly research.

I further authorize Ryerson University to reproduce this dissertation by photocopying or by other means, in total or in part, at the request of other institutions or individuals for the purpose of scholarly research.

I understand that my dissertation may be made electronically available to the public. 


\title{
Understanding PIKfyve control of lysosome dynamics
}

\author{
Golam T. Saffi \\ Doctor of Philosophy, 2019 \\ Molecular Science, Ryerson University
}

\begin{abstract}
:
Lysosomes are organelles that receive external cargo through phagocytosis and endocytosis, and internal cargo through autophagy, followed by degradation in the acidic and hydrolase rich lumen and redistribution of substrates for maintaining cellular integrity. Lysosomes undergo homotypic or heterotypic repeated fusion and fission or kiss and run cycles with other organelles to exchange and receive cargo, as well as maintain lysosome number and size. Lysosome membranes display the phosphoinositide lipid phosphatidylinositol 3,5bisphosphate (PtdIns(3,5) $\left.\mathrm{P}_{2}\right)$ synthesized by the lipid kinase PIKfyve. PtdIns(3,5) $\mathrm{P}_{2}$ act as a signalling lipid on lysosomes to regulate maturation of endosomes, phagosomes and autophagosomes maturation by fusing with lysosomes, and recycling from the lysosomal lumen, lysosome ion channel activity, and lysosome-associated actin turnover. Of these defects, the most dramatic phenotype of PtdIns(3,5) $\mathrm{P}_{2}$ depletion from PIKfyve inhibition is the appearance of enlarged lysosomes. Our work demonstrates that $\operatorname{Ptd} \operatorname{Ins}(3,5) \mathrm{P}_{2}$ is an important regulator of lysosome size and number by governing the balance between lysosome fusion and fission and/or kiss and run. Depletion of $\operatorname{PtdIns}(3,5) \mathrm{P}_{2}$ arrests lysosome fission disrupting the balance between the continuous fusion and fission cycle, leading to lysosome coalescence and causing lysosome enlargement and reduction in their numbers. Microtubules, cytoskeletal tracks for lysosome
\end{abstract}


positioning, and associated motor protein complexes, kinesin-1 and dynein, regulate lysosome coalescence during PIKfyve inhibition.

Our experimental observations revealed ROS as a novel regulator of lysosome fusion and fission. Specifically, ROS arrested lysosome enlargement from acute PIKfyve inhibition and accelerated lysosome fragmentation during PIKfyve re-activation. However, depending on the ROS produced and/or site of ROS synthesis, lysosome dynamics are affected distinctly. $\mathrm{H}_{2} \mathrm{O}_{2}$ impaired lysosome mobility to arrest coalescence. However, superoxide generated from mitochondrial ETC complex 1, or thioredoxin reductase, or glutathione inhibition through rotenone, or CDNB, or MCB respectively depolymerised microtubules without affecting mobility. Instead, superoxide generation through pharmacological manipulations promoted actin clearance from lysosomes, which otherwise accumulate on lysosomes to hinder fission upon PIKfyve inhibition, to promote fission. Indeed, actin depolymerisation arrested lysosome enlargement during acute PIKfyve inhibition and accelerated lysosome fragmentation during PIKfyve re-activation, further indicative of ROS stimulating lysosome fission through actin clearance. 


\section{Acknowledgements}

First, I would like to thank Allah, for granting me guidance and understanding of life in general and to set the circumstances for me to survive during my PhD graduate studies. Next, I would like to thank my supervisor, Dr. Roberto Botelho, for his immense support and academic mentorship during my five years of $\mathrm{PhD}$ graduate studies. We had some disagreements at the beginning regarding me failing the $\mathrm{PhD}$ candidacy examination, not handling the HPLC machine and the hot room equipment properly. Yes, I still remember. Nonetheless, his support enabled me to grow academically and professionally as a cell biologist. Additionally, he did not show any sense of lack of confidence in me as I had 3 kids during my $\mathrm{PhD}$ years. Also, I would like to thank my wife, Mumtahina Biswas, who is a blessed partner for which I am grateful, who I share responsibility to sustain our family, and also whose perseverance and commitment was also a source of strength for me. I would also like to thank my mom and dad for their support. Lastly, I would like to thank previous and current lab peeps, Dr. Christopher Choy, Evan Tang, Dr. Matthew Gray, Shannon Ho, Victoria Hipolito, Aaron Fountain, and Krishna Chintaluri, whose professional support and conversations made the experience enjoyable at the lab. 


\section{Table of contents:}

$\begin{array}{lll}\text { Abstract } & \text { ii }\end{array}$

List of figures $\quad$ iv

List of appendices $\quad$ xiv

List of abbreviations $\quad$ XV

Chapter 1 Introduction 1

1.1 The endomembrane system 2

1.1.1 The endosomal pathway 3

1.1.1.1 Lysosomes as terminal organelles of the endosomal pathway 3

1.1.1.2 Endocytosis and endosome maturation 4

$\begin{array}{lll}\text { 1.1.2 Lysosomes } & 7\end{array}$

$\begin{array}{lll}\text { 1.1.3 Membrane trafficking to lysosomes } & 10\end{array}$

1.1.3.1 Microtubules structure and motors $\quad 10$

1.1.3.2 Microtubule motor protein complexes in cargo traffic $\quad 11$

1.1.3.3 Microtubules as dynamic regulators of lysosome traffic $\quad 14$

1.1.4 SNARE proteins and membrane fusion 16

1.1.4.1 SNAREs as dynamic mediators of lysosome membrane fusion 18

1.1.5 Lysosome membrane fission 19

1.2 GTPase and phosphoinositide regulation of membrane trafficking 22

1.2.1 GTPase structure and function 23

1.2.2 Phosphoinositide structure and regulation 24

1.2.2.1 Co-ordination between GTPases and phosphoinositides for signaling 28 
1.2.3 Phosphoinositide signaling regulate endosomal maturation and membrane remodelling

1.2.4 PtdIns $(3,5) \mathrm{P}_{2}$ regulate lysosome signaling and membrane remodelling

1.2.4.1 Lipid kinase PIKfyve and PtdIns(3,5) $\mathrm{P}_{2}$ synthesis

1.2.4.2 PtdIns(3,5) $\mathrm{P}_{2}$ signaling at the lysosome membrane and membrane remodelling

1.2.4.3 PtdIns(3,5) $\mathrm{P}_{2}$ and pathophysiology

1.3 Lysosome membrane fission dynamics during autophagy, phagocytosis and endocytosis

1.3.1 Endolysosome membrane remodelling and lysosome reformation

1.3.1.1 Lysosome kiss and run

1.3.1.2 PtdIns(4)P co-ordinate with coat proteins for lysosome membrane reformation

1.3.1.3 PtdIns(3,5) $\mathrm{P}_{2}$ regulate lysosome membrane reformation

1.3.1.4 Microtubules and actin exert mechanical force for lysosome membrane fission

1.3.2 Autolysosome membrane remodeling and lysosome reformation

1.3.2.1 Autophagosome initiation and maturation

1.3.2.2 Membrane remodeling and vesiculation during ALR

1.3.3 Phagolysosome membrane remodelling and lysosome reformation

1.3.3.1 Phagocytosis initiation and maturation

1.3.3.2 Phagolysosome membrane remodelling and lysosome reformation 
1.4.1 Types of ROS $\quad 50$

1.4.2 Synthesis of ROS

1.4.3 Harmful effects of ROS $\quad 54$

1.4.4 Anti-oxidant systems to circumvent ROS production 56

1.4.5 Beneficial impact of ROS

1.4.6 ROS regulate lysosome function and dynamics 58

$\begin{array}{ll}1.5 \text { Research objective } & 61\end{array}$

$\begin{array}{lll}\text { 1.5.1 Hypothesis I } & 61\end{array}$

$\begin{array}{ll}\text { 1.5.1.1 Objectives } & 62\end{array}$

$\begin{array}{lll}\text { 1.5.2 Hypothesis II } & 64\end{array}$

1.5.2.1 Objective $\quad 64$

$\begin{array}{ll}\text { Chapter 2 Materials and methods } & 66\end{array}$

Chapter 3 Results: Lysosome enlargement during PIKfyve inhibition occurs due 76

to coalescence

$\begin{array}{ll}3.2 \text { Introduction } & 77\end{array}$

$\begin{array}{ll}3.2 \text { Results } & 78\end{array}$

3.2.1 PtdIns $(3,5) \mathrm{P}_{2}$ depletion reduce lysosome number and increase average

$\begin{array}{ll}\text { lysosome volume } & 78\end{array}$

3.2.2 PIKfyve reactivation increase lysosome number and reduce individual

$\begin{array}{lr}\text { lysosome volume } & 80\end{array}$

3.2.3 Lysosome undergo constant "kiss-and-run" under regulation by $\operatorname{Ptd} \operatorname{Ins}(3,5) \mathrm{P}_{2} \quad 82$ 
3.2.4 Lysosome dynamics during PIKfyve inhibition and re-activation is microtubule $\begin{array}{ll}\text { dependent } & 86\end{array}$

3.2.5 Microtubule motor complexes regulate lysosome coalescence during $\begin{array}{ll}\text { PIKfyve inhibition } & 88\end{array}$

Chapter 4 Results: Reactive oxygen species regulate lysosome fusion-fission 91

$\begin{array}{ll}4.1 \text { Introduction } & 92\end{array}$

$\begin{array}{ll}4.2 \text { Results } & 93\end{array}$

4.2.1 Frequent laser excitation from fluorescence microscopy produce ROS 93

4.2.2 ROS stimulation arrest lysosome enlargement from acute PIKfyve inhibition 94

4.2.3 ROS stimulation arrest lysosome enlargement from acute PIKfyve inhibition

without neutralizing apilimod or enhancing PtdIns(3,5) $\mathrm{P}_{2}$ synthesis 99

4.2.4 ROS increase lysosome fragmentation upon apilimod removal 102

$\begin{array}{ll}\text { 4.2.5 ROS affect microtubule structure } & 104\end{array}$

4.2.6 Distinct ROS regulation of lysosome movement 105

4.2.7 ROS does not stimulate lysosome fission through clathrin or dynamin $\quad 110$

4.2.8 ROS remove actin from lysosomes to prevent coalescence $\quad 115$

$\begin{array}{lr}\text { Chapter } 5 \text { Discussion } & 119\end{array}$

5.1 Lysosome enlargement due to inhibition of the lipid kinase PIKfyve occurs

$\begin{array}{lr}\text { through coalescence } & 120\end{array}$ 
5.1.1 Lysosomes coalesce from PtdIns(3,5) $\mathrm{P}_{2}$ depletion due to disrupted

lysosome fusion-fission cycle

5.1.2 Microtubules regulate $\operatorname{Ptd} \operatorname{Ins}(3,5) \mathrm{P}_{2}$ dependent lysosome fusion-fission

5.1.3 Lysosome coalescence from acute PIKfyve inhibition is independent

of protein biosynthesis

5.1.3.1 TFEB activation from acute PIKfyve inhibition does not contribute

to lysosome coalescence

5.1.4 Future directions

5.2 ROS regulate lysosome fusion-fission dynamics during PIKfyve inhibition

5.2.1 ROS prevent lysosome enlargement during acute PIKfyve inhibition and accelerate fragmentation during PIKfyve re-activation

5.2.2 ROS affect lysosome fusion-fission dynamics distinctly

5.2.2.1 $\mathrm{H}_{2} \mathrm{O}_{2}$ effect on lysosome enlargement and fragmentation may occur by disrupting lysosome motility

5.2.2.2 Rotenone, $\mathrm{CDNB}$ and $\mathrm{MCB}$ effect on lysosome enlargement

and fragmentation 
Appendices

References 


\section{List of figures:}

Figure 1: Lysosomes receive cargo from outside and inside cell

Figure 2: Microtubule structure and organization

Figure 3: SNAREs in heterotypic membrane fusion

Figure 4: General dynamics of lysosome fission

Figure 5: General structure of phosphatidylinositol

Figure 6: Lipid membranes display numerous phosphoinositide species

Figure 7: General mechanism of lysosome budding and tubulation

Figure 8: Mechanism of ROS production

Figure 9: Possible mechanisms for lysosome enlargement during $\operatorname{Ptd} \operatorname{Ins}(3,5) \mathrm{P}_{2}$

depletion

Figure 10: PtdIns(3,5) $\mathrm{P}_{2}$ depletion reduce lysosome number and increase

average volume in RAW macrophages

Figure 11: Apilimod treatment reduce lysosome number and increase

average volume in HeLa cells

Figure 12: PIKfyve reactivation increase lysosome number and reduce

average volume

Figure 13: Spinning disc live cell imaging with frequent laser 
exposure impair lysosome movement

Figure 14: Spinning disc live cell imaging with low frame rate laser

exposure to capture apilimod induced lysosome vacuolation

Figure 15: Swept field confocal live imaging to capture

apilimod induced lysosome enlargement

Figure 16: Microtubule regulate lysosome dynamics during

PIKfyve inhibition and reactivation

Figure 17: Microtubule motors regulate apilimod induced

lysosome enlargement

Figure 18: Microscopy induced photo-damage causes ROS production

Figure 19: ROS stimulation through pharmacological manipulation

Figure 20: ROS agonists prevent lysosome enlargement during acute

PIKfyve suppression

Figure 21. ROS scavengers permit lysosome coalescence during acute

PIKfyve suppression

Figure 22: ROS prevent lysosome enlargement during acute PIKfyve

suppression in HeLa and RPE cells

98

Figure 23: Apilimod integrity and $\operatorname{PtdIns}(3,5) \mathrm{P}_{2}$ levels are not altered by ROS

101 
Figure 24. ROS accelerate recovery of lysosome size and number upon

PIKfyve reactivation

103

Figure 25. ROS agents differentially affect the microtubule system

105

Figure 26: Distinct ROS agents differentially impact lysosome motility

Figure 27: Increased microtubule stability does not affect lysosome

motility or lysosome coalescence during PIKfyve inhibition

108

Figure 28. ROS do not affect Rab7 activation and Arl8b loading onto

lysosomes

Figure 29. $\mathrm{H}_{2} \mathrm{O}_{2}$ boosts recruitment of clathrin and dynamin to membranes

Figure 30: Clathrin inhibition does not arrest ROS-mediated lysosome

fragmentation during PIKfyve reactivation

Figure 31. Dynamin inhibition does not affect lysosome fragmentation

during during PIKfyve reactivation

114

Figure 32: ROS promote actin clearance to rescue lysosome coalescence during PIKfyve inhibition and accelerate fragmentation during PIKfyve

reactivation

Figure 33: Microtubule depolymerisation affect lysosome "kiss-and-run"

during PIKfyve inhibition and reactivation 


\section{List of Appendices}

Supplemental Figure A1: PIKfyve inhibition cause nuclear translocation of TFEB

Supplemental Figure B1: PIKfyve and mTOR function independently

Supplemental Figure C1: Acute PIKfyve inhibition increase expression of

lysosome genes without affecting protein expression

Supplemental Figure D1: TFEB and protein synthesis do not contribute to

lysosome enlargement 


\title{
List of abbreviations
}

\author{
ER \\ endoplasmic reticulum \\ ATP \\ adenosine triphosphate \\ LAMP1 \\ lysosome-associated membrane protein 1 \\ GTP \\ guanosine triphosphate \\ EEA1 \\ early endosome antigen 1 \\ ESCRT \\ endosomal sorting complexes required for transport \\ SNARE \\ soluble N-ethylmaleimide-sensitive factor attachment protein receptors \\ MT \\ microtubule \\ RILP \\ Rab-interacting lysosome protein \\ HOPS \\ homotypic fusion and protein sorting complex \\ PtdInsP \\ phosphoinositide \\ PtdIns phosphatidylinositol \\ PtdIns(3)P phosphatidylinositol 3-phosphate \\ PtdIns(4)P phosphatidylinositol 4-phosphate \\ PtdIns(3,5) $\mathrm{P}_{2} \quad$ phosphatidylinositol 3,5-bisphosphate \\ PtdIns(4,5) $\mathrm{P}_{2} \quad$ phosphatidylinositol 4,5-bisphosphate \\ TRPML1 \\ transient receptor potential cation channel, mucolipin subfamily
}


mTORC1 mammalian target for rapamycin complex 1

ROS reactive oxygen species

TFEB transcription factor EB

CDNB 1-chloro-2,4-dinitrobenzene

MCB monochlorobimane

ETC mitochondrial electron transport chain

F-actin filamentous actin 
Chapter 1: Introduction 


\subsection{Introduction:}

\subsection{The endomembrane system}

Enclosed within the plasma membrane, eukaryotic cells house multiple membrane-less and membrane-bound organelles, which are loosely defined as subcellular environments in which specific functions occur. Among organelles include ribosomes which are protein-RNA complexes, signalosomes which are protein complexes, and lipid membrane-bound structures such as endosomes, lysosomes, and mitochondria. Membrane-bound organelles can be further classified as: single lipid layer enclosed structures such as lipid droplets, single phospholipid bilayer structures such as lysosomes and double phospholipid bilayer structures such as autophagosomes (Satori et al., 2013). Examples of some functions occurring within organelles include protein folding in the ER, ATP production in the mitochondria as energy resource, protein synthesis from cytosolic or ER-bound ribosomes, DNA storage and transcription within the nucleus, macromolecule and pathogen degradation within the lysosome (Ba et al., 2018; Heald and Cohen-Fix, 2014). Of the various organelles present within a mammalian cell, lysosomes serve as crucial organelles of the endosomal pathway. They are highly dynamic through homotypic or heterotypic membrane fusion with late endosomes, autophagosomes, and phagosomes for exchange of luminal contents and cargo reception, followed by lysosome reformation or fission (Saffi and Botelho, 2019). Understanding lysosome membrane fusion and fission, as well as the importance and governing factors regulating such processes will be the main focus of my dissertation. 


\subsubsection{The endosomal pathway}

\subsubsection{Lysosomes as terminal organelles of the endosomal pathway}

Lysosomes receive cargo internalized through endocytosis for degradation. However, lysosomes are not homogenous in hydrolytic properties. Instead, lysosomes can be classified into heterogeneous and functionally discrete organelles. "Terminal lysosomes" can refer to nonhydrolytic and non-acidic store of hydrolytic enzymes. "Endolysosomes" refer to hybrid organelles between late endosome and terminal lysosome. "Endo/lysosome" to refer to undefined mixture of late endosomes, lysosomes and endolysosomes which occur due to lack of proper distinction between these organelles, when LAMP1 (lysosomal-associated membrane protein 1) marker do not work to differentiate these organelles. "Proto-lysosome" to refer to immature lysosomes that reform or separate from autolysosomes, that do not house cargo or lysosome hydrolases, but possess lysosome membrane proteins (Bissig et al., 2017; Bright et al., 2016; Klumperman and Raposo, 2014; Zeigerer et al., 2012).

The classification of lysosomes is complicated further due to the existence of lysosomerelated organelles (LROs), which are formed through interaction with existing lysosomes or possess similar to lysosome biogenesis machinery. Examples of organelles with similar lysosome biogenesis machinery include lytic granules from cytotoxic T lymphocytes, primary granules in neutrophils, and dendritic cell housed major histocompatibility complex II (MHC-II) compartment. Additionally, during phagocytosis, endocytosis, and autophagy, lysosomes interact and mature through fusion with phagosomes, late endosomes and autophagosomes to form phagolysosomes, endolysosomes and autolysosomes respectively (Hipolito et al., 2018; Klionsky et al., 2014; Marks et al., 2013). Overall, lysosomes are functionally heterogeneous performing 
diverse roles in immunity and nutrient acquisition, and my work will focus on lysosome dynamics along the endosomal pathway.

\subsubsection{Endocytosis and endosome maturation}

Endocytosis is a process involving uptake of macromolecules from the extracellular fluid along with plasma membrane integral proteins and lipids through plasma membrane remodelling and invagination to form early endosomes that mature into late endosomes and endolysosomes through late endosome-lysosome fusion for cargo degradation and substrate redistribution for maintaining cellular integrity. Endocytosis can occur through interaction of cargo receptors with plasma membrane associated clathrin coat protein, or through clathrin-independent passage which involves lipid rafts (Bissig et al., 2017; Elkin et al., 2016). Examples of cargo internalized through endocytosis include receptor-ligand complexes of growth factors, nutrient transporters, lipids, cholesterol, and extracellular materials including pathogens. Failure to internalize and sort endocytic cargo can be detrimental to cells and organism - for example, the low-density lipoprotein (LDL) carries dietary cholesterol and is endocytosed via the LDL receptor, where it traffics to lysosomes for degradation and release of cholesterol - cholesterol is a component of the membranes, steroids and bile acids. However, endocytosis of LDL followed by impaired export of cholesterol released by LDL degradation in lysosomes can lead to cardio-vascular disease and the neurodegenerative Niemann-Pick (NPC) disease (Bi and Liao, 2010; Goldstein et al., 1982; Huber and Teis, 2016).

Clathrin-mediated endocytosis involves recognition of transmembrane receptors and their ligands by cargo adaptors followed by assembly by clathrin coat proteins on the plasma 
membrane into clathrin-coated pits (CCPs) and subsequent invagination of the plasma membrane for internalization as clathrin-coated vesicles (CCVs). Cargo recognition during endocytosis maybe mediated by the adaptor AP-2 complex or AP-2 independent mechanisms. AP-2 recognizes dileucine and tyrosine motifs on cargo. AP-2 recognition of cargo connect the cargo to clathrin. Following vesicle formation, dynamin GTPase assemble at the constricted neck of the vesicle and stimulates membrane scission through GTP hydrolysis. Vesicle release is accompanied by dissociation of clathrin from the vesicle through the co-ordination of auxillin co-factor and ATPase activity of hsc70, followed by delivery of cargo through the endocytic pathway towards lysosomes (Chang et al., 2002; Doherty and McMahon, 2009; Motley et al., 2003).

Clathrin-independent endocytosis include caveolae-mediated endocytosis. Caveolae are invaginations of the plasma membrane rich in cholesterol, sphingolipids and the protein caveolin, and such invaginations form lipid rafts. Mechanistically, EHD2 (EH-domain containing protein) assembles around the neck of the caveolae through oligomerization. This is followed by recruitment of pacsin 2 by EHD2, where pacsin 2 induces membrane curvature through F-BAR domain and recruits dynamin-2 to stimulate membrane scission (Bastiani and Parton, 2010; Cheng and Nichols, 2016; Nabi and Le, 2003). Furthermore, macropinocytosis, a process by large gulps of extracellular fluid is internalized, is another mode of clathrin-independent endocytosis (Sandvig et al., 2018).

Following endocytosis, vesicles and cargo are delivered to early endosomes, or which there are several types. Rab5-positive early endosomes also display Rab4, Arf1, and to a smaller extent Rab7 GTPase. Rab5-positive early endosomes interact with one or both of PtdIns(3)Pnegative APPL1 (adaptor protein containing PH domain, PTB domain and leucine zipper motif 
1) early endosomes that mature into PtdIns(3)-positive EEA1 early endosomes. This result in physically distinct endosomes identified by EEA1 or APPL1, that exchange content and sort cargo for either recycling to the plasma membrane, trafficking to the Golgi network, or degradation in lysosomes (Schmid, 2017; Vonderheit and Helenius, 2005; Zoncu et al., 2009).

Early endosomes serve as platforms to initiate cargo recognition and recruitment for internalization within lysosomes for eventual degradation. PtdIns(3)P on early endosomes sequentially recruit ESCRT-0/1/2/3 complexes, which invaginate early endosome membranes followed by membrane scission through the ATPase activity of Vps4, resulting formation of intraluminal vesicles (ILVs). This is accompanied by Rab5-GTP dependent recruitment of Rab7 followed by loss of Rab5-GTP, Rab7 dependent ILV formation due to interaction of Rab7-GTP effector RILP with ESCRT-II for membrane recruitment, causing early endosomes to mature into late endosomes. Late endosomes, filled with intraluminal vesicles housing cargo from early endosomes and lysosome hydrolases imported from Golgi, fuse with lysosomes resulting into endolysosomes, for cargo degradation and hydrolase delivery (Huotari and Helenius, 2011; Wang and Hong, 2006). Following cargo degradation in endolysosomes, lysosomes reform by separating from endolysosomes to maintain lysosome number and size, and maintain autophagic, phagocytic and endocytic flux as needed. The dynamics of lysosome homotypic or heterotypic fusion with other organelles will be discussed further, including the concept of full fusion and fission versus kiss-and-run. 


\subsubsection{Lysosomes}

Lysosomes serve as terminal organelles that can receive cargo from outside the cell through phagocytosis and endocytosis as well as from inside the cell through autophagy to degrade organelles, defective cells, pathogen and macromolecules for immunity, pathogen clearance, maintaining cell integrity, metabolism and cargo recycling (Pu et al., 2016) (Fig. 1). Lysosomes are membrane enclosed organelles, which are acidic due to proton pumping activities of membrane bound V-ATPase complex, which utilizes ATP hydrolysis, to acidify the lumen for a pH of 4.6-5.0 (Luzio et al., 2007; Mindell, 2012). Furthermore, lysosomal lumen house various hydrolases, which are active at acidic $\mathrm{pH}$ of lumen, consisting of proteases, nucleases, lipases, glycosidase, sulfatase, phospholipase, and phosphatase, that work in concert for macromolecular or cargo degradation (Ba et al., 2018; Mindell, 2012). Over the years, the concept of a lysosome changed from just a "waste bag" that can degrade and recycle cellular waste, to a "control centre" of cell metabolism due to identification of lysosome to nucleus signalling pathways and lysosome gene network that regulate energy metabolism and cell clearance (Ballabio, 2016). 


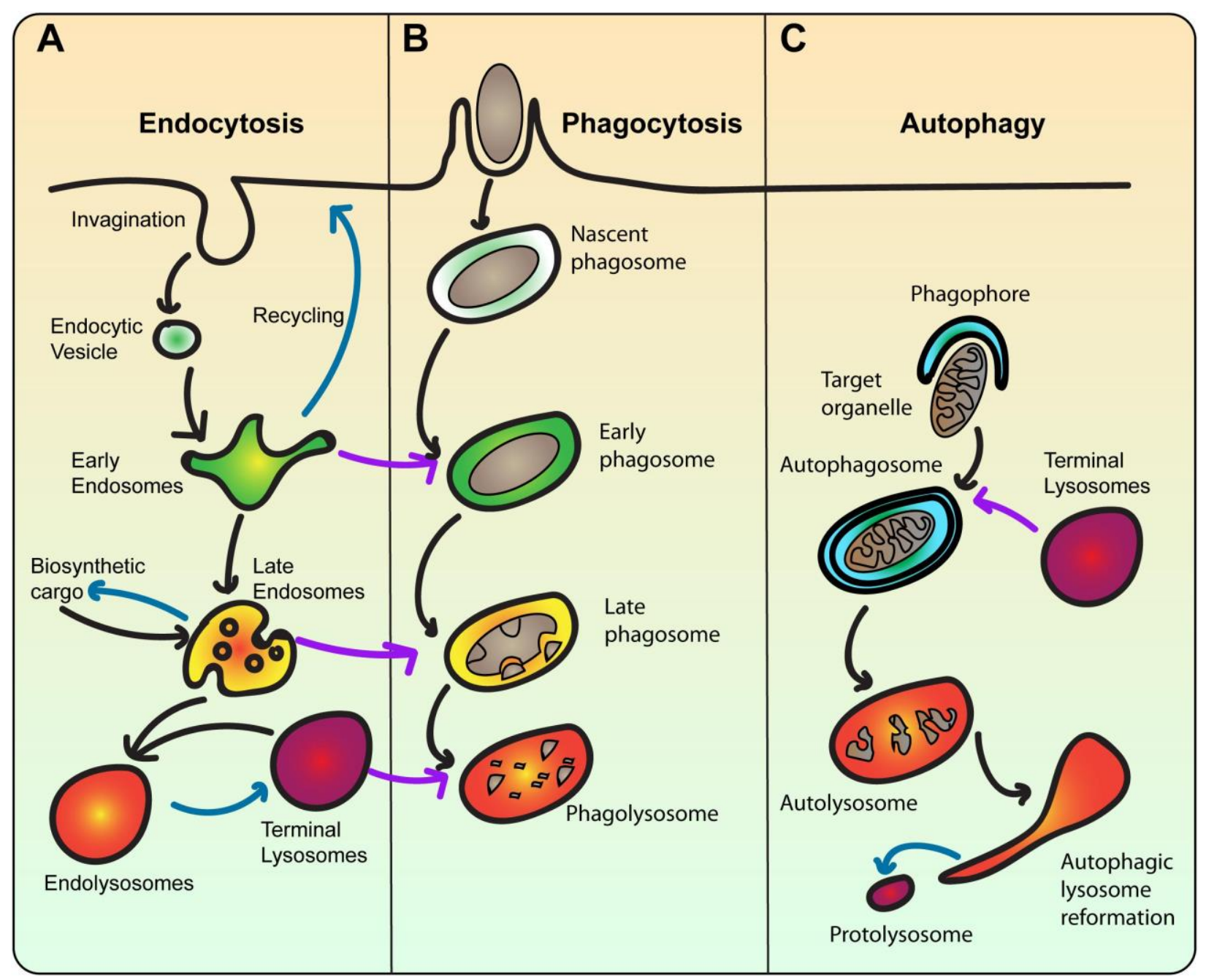

Figure 1: Lysosomes receive cargo from outside and inside cell. A) Endocytosis proceeds with cargo recognition by cell surface receptors that are internalized through plasma membrane invagination and vesiculation. Endocytic vesicles fuse with early endosomes that sort cargo for recycling, or proceeding towards lysosomes through maturation of early into late endosomes. Such maturation consists of formation of intraluminal vesicles present in late endosomes. Late endosomes also acquire Golgi-derived lysosome proteases. Late endosomes filled with endocytic cargo and Golgi-derived lysosome proteins fuse with terminal lysosomes, non-acidic lysosomes with hydrolytic enzymes, to form endolysosome for cargo degradation. Terminal lysosomes may reform from endolysosomes. (B) Phagocytosis proceeds with internalization of extracellular 
particles such as pathogenic bacteria, causing plasma membrane remodelling and curvature to form phagosomes. Phagosomes fuse with early and late endosomes and finally lysosomes to form phagolysosomes for cargo degradation. (C) Autophagy proceeds with sequestration of intracellular surplus or damaged organelles into double bilayered autophagosome, where the double-bilayer is derived from ER. Autophagosomes mature into autolysosome through autophagosome-lysosome heterotypic membrane fusion. Following cargo degradation, autolysosomes undergo autophagic lysosome reformation (ALR) for membrane remodelling and tubulation, followed by membrane scission to form proto-lysosomes, which are non-acidic and absent of lysosome hydrolases but possess membrane proteins (Inpanathan and Botelho, 2019).

Lysosomes are highly dynamic and differ in number according to different cells or even within the same cell type, and their shape can dramatically transform from punctate to tubular depending on the stimulus that results in such a phenotype. Lysosomes may also vary in $\mathrm{pH}$, motility, degradative capability, and position. Punctate shaped vesicular lysosomes can have a diameter between $0.5 \mu \mathrm{m}$ to over $1 \mu \mathrm{m}$, whereas tubular lysosomes can extend to beyond $15 \mu \mathrm{m}$ (Ba et al., 2018; Heuser and Supp, 1989; Hipolito et al., 2018; Noack et al., 2018). Lysosomes also differ in motility depending on their distribution, where perinuclear distributed seem less motile but more able to receive cargo and more acidic compared to peripheral lysosomes (Pu et al., 2016).

Overall, despite this diversity and complex nature of lysosomes, these organelles are highly dynamic. They constantly acquire endocytic, autophagic and biosynthetic cargo and must also undergo recycling and fission to maintain number and size. Next, I will discuss mechanisms of lysosome fusion, including the concept of full fusion versus "kiss-and-run". 


\subsubsection{Membrane trafficking to lysosomes}

Membrane trafficking toward lysosomes requires several steps. First, cargo vesicles must be labelled with molecular identity that defines this as cargo destined for degradation. This can include the acquisition of specific Rab and Arf-family GTPases as well as phosphoinositide lipids (defined later), which recruit variety of effector proteins. These proteins then are able to interact with microtubule-based motors to traffic these towards cell periphery or cell centre where they encounter lysosomes. This is followed by engaging tethering factors that capture target vesicles to lysosomes, and ultimately engaging the SNARE (soluble N-ethylmaleimidesensitive factor attachment protein receptors) machinery that drives actual membrane fusion. Ultimately, membranes must be recycled and lysosomes reformed to ensure continuous membrane trafficking and flux. I will next elaborate on the microtubule system and SNARE machinery before delving further into regulators of lysosome identity, function and trafficking.

\subsubsection{Microtubules structure and motors}

Microtubules serve as motility tracks for various organelles including lysosomes. They regulate the rate by which lysosomes encounter and fuse with target organelles like late endosomes, phagosomes and autophagosomes (Storrie and Desjardins, 1996). Microtubules (MTs) are part of the cytoskeleton network and are formed from heterodimers of $\alpha$ - and $\beta$-tubulin monomers that undergo polymerization. MTs are organized with $\beta$-tubulin exposed at plus ends and $\alpha$-tubulin exposed at minus ends from $\alpha / \beta$-tubulin heterodimers to form protofilaments; it takes 13 protofilaments aligned in parallel to form a microtubule (Ti et al., 2018). MT assembly requires a nucleating template, usually in the form of the $\gamma$-tubulin ring complex ( $\gamma$ TuRC). 
However, MT nucleation may also occur independently of $\gamma \mathrm{TuRC}$, as suggested from observations of MT activity in $\gamma$-tubulin depleted models (Tovey and Conduit, 2018).

MTs are highly dynamic and undergo cycles of growth and shrinkage as "dynamic instability", thus regulating cargo traffic through dynamic alteration of the MT track. During MT growth, the plus end consisting of $\beta$-tubulin expose GTP that receive incoming $\alpha$-tubulin from $\alpha / \beta$-tubulin heterodimer, causing GTP hydrolysis and formation of GTP cap from the growing end of MT (Roostalu and Surrey, 2017; Yogev et al., 2016). MT shrinkage occurs due to shift between GTP-bound tubulin, which promotes association and extension, and GDP-bound tubulin due to GTP hydrolysis, which causes deformation of the tubulin subunit to a curved state (Fig. 2). This curved GDP-tubulin end displays lower affinity for other tubulin subunits, begins to come apart and splaying continues on down the microtubule, thereby causing microtubule depolymerization (Burbank and Mitchison, 2006; Tamariz and Varela-Echavarrã-a, 2015; Zhai et al., 1996).

\subsubsection{Microtubule motor protein complexes in cargo traffic}

Cargo motility along MT tracks is controlled by motor protein complexes, dynein and kinesin, that use ATP hydrolysis to transport organelles such as endosomes, lysosomes and mitochondria to minus end and plus end respectively. There are 14 subfamilies of kinesin from kinesin-1 to kinesin-14, with kinesin-1 being involved in lysosome movement. Dynein classified as cytoplasmic or axonemal. Cytoplasmic dynein drives cytoplasmic cargo such as lysosomes, endosomes, phagosomes or vesicular transport within the Golgi network, while axonemal dynein is involved in cilia motility for transport of mucus fluid in the lungs. Cytoplasmic dynein are thus 
involved in lysosome mobility. In general, dynein through minus-end transport drive cargo to the perinuclear area of the cell. Kinesin-1 through plus-end transport drive cargo to the cell periphery (Gennerich and Vale, 2009; Guardia et al., 2016; Pfister et al., 2006; Xiao et al., 2016).

Structure of microtubule motors are organized to couple cargo recognition with ATP hydrolysis for driving cargo motility across microtubules. Structurally, cytoplasmic dynein consists of two heavy chains and several smaller subunits. The heavy chains consist of four domains: tail, linker, head and stalk (Fig. 2). The tail at the N-terminus $~ 1400$ amino acids bind cargo, followed by linker $\sim 550$ amino acids functions to amplify translocation of dynein across microtubule through ADP binding, head consist of six tandem AAA+ modules where the first AAA module functions as ATPase in ATP hydrolysis to provide energy for dynein movement, and C-terminal stalk domain bind to microtubules (Burgess et al., 2003; Kato et al., 2018; Kikkawa, 2013; King, 2016).

Kinesin-1 exists as heterotetramer consisting of two kinesin heavy chains (KHC) as a homodimer bound to two kinesin light chains (KLC). KHC consists of an N-terminal head motor domain that binds MT and couples cargo recognition with ATPase activity as the energy input for motor activity; this is followed by a neck linker domain that connects the head to the coiled coil stalk domain. The neck domain has been proposed to drive motor movement along microtubules through docking onto the head in ATP bound state and undock after ATP hydrolysis, thereby switching between the two head domains through cycles of docking and undocking to cause motor "walking" along MTs. The alpha helix coiled-coil stalk mediates KHC protein homodimerization and co-ordinates the head for motor movement along microtubules. Following the coiled coil stalk, the tail domain interacts with cargo (Endow et al., 2010; Rice et al., 1999; Thormä et al., 1998; Wang et al., 2015). KLC consists of an N-terminal heptad repeat 
that oligomerizes with the KHC stalk, the linker domain with acidic residues, and six TPRs (tetratricopeptide repeats) that interact with Leu-Phe-Pro (LFP) from the linker domain that are destabilized upon cargo binding to relieve kinesin-1 from autoinhibited state for motor activity (Cockburn et al., 2018). Thus, the head, neck linker, stalk and tail domains from the KHC coordinate and use ATP hydrolysis to drive motor complex movement along microtubules upon cargo recognition from the KLC (Fig. 2).

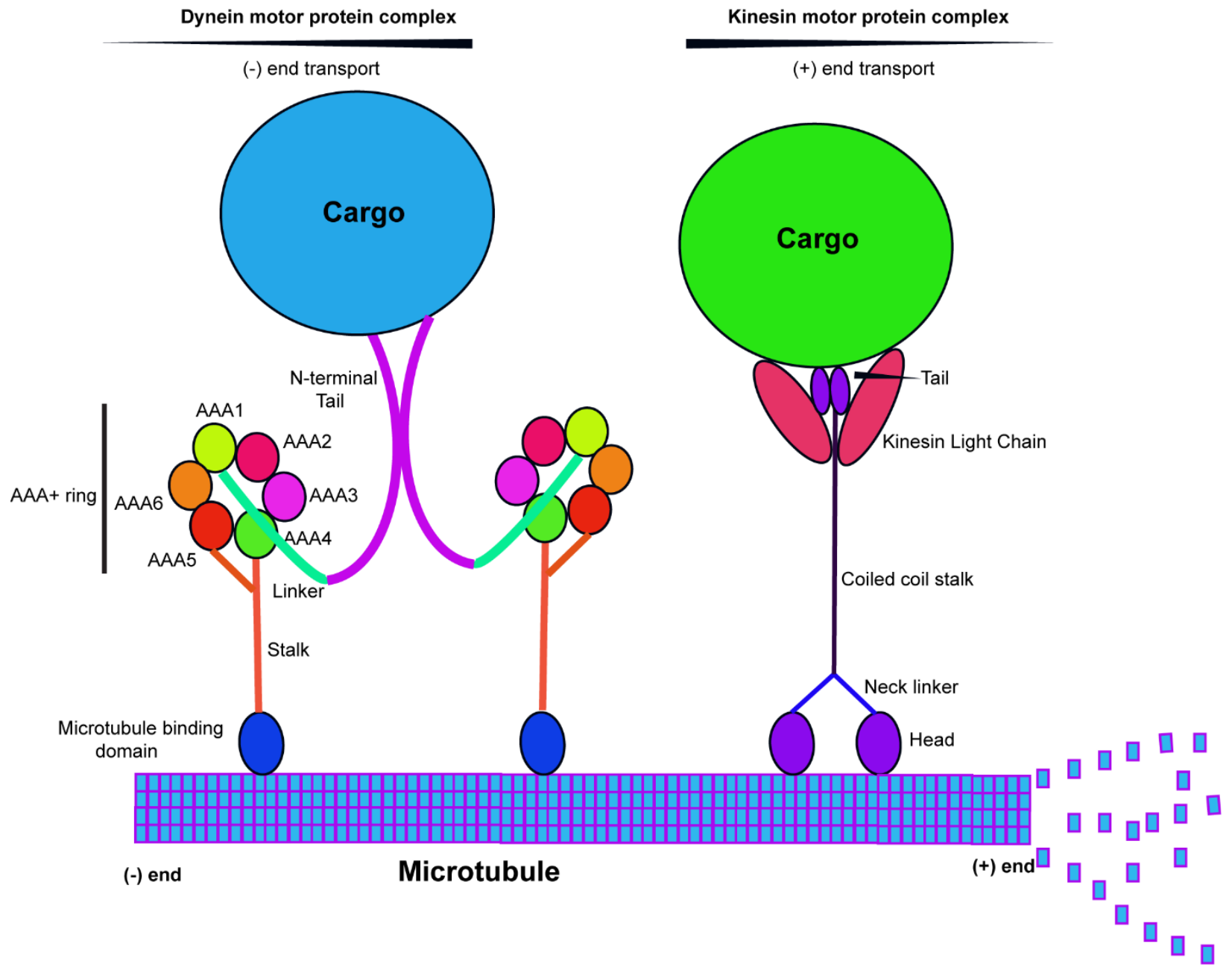

Figure 2: Microtubule structure and organization. Microtubules consist of $\alpha / \beta$-tubulin dimers forming protofilaments that are laterally associated. Microtubule growth or shrinkage occurs at 
(+) end from GTP or GDP-bound tubulin respectively. Shrinkage occurs due to curvature of GDP-tubulin and splaying down for depolymerisation. Microtubules are attached to motor protein complexes: cytoplasmic dynein that carry cargo towards the (-) end and kinesin-1 that move cargo along the (+) end. Cytoplasmic dynein complex consist of four domains: N-terminal tail for cargo recognition, linker that interact with AAA1 module of head for amplification of dynein mechanical force, head with AAA+ modules for ATP hydrolysis, and stalk for microtubule binding. Kinesin-1 consist of two heavy chains (KHC) and two light chains (KLC). KHC consist: two head motor domains for ATP hydrolysis and microtubule walking, neck linker domain for co-ordinating motor movement, coiled coil stalk for KHC protein homodimerization, and tail for cargo recognition. KLC consist: heptad repeat for oligomerization with KHC, linker domain that relieve KLC from autoinhibited state upon interaction with TPRs due to TPR interaction with cargo.

\subsubsection{Microtubules as dynamic regulators of lysosome traffic}

Microtubules are important regulators of organelle traffic by facilitating contact between two opposing organelles and ultimately fusion. For example, during autophagy, which is a stress response to nutrient deprivation, microtubules and their motors catalyse autophagosome movement from peripheral to perinuclear sites towards lysosomes found at the microtubule organizing center (Pu et al., 2016). This is followed by either microtubule-dependent complete fusion of autophagosomes with lysosomes, or "kiss-and-run" between autophagosomes and lysosomes, where lysosomes upon contact form a temporary fusion pore with autophagosomes to exchange content, followed by separation of the lysosome and autolysosome (Jahreiss et al., 2008). 
Other examples include macropinosomes formed during macropinocytosis, a process by which cells uptake extracellular fluid; after formation, macropinosomes engage microtubules and their motors to fuse with endosomes and lysosomes for processing of fluid components (Clarke et al., 2002). In addition, phagosomes formed during phagocytosis, which involves the internalization of particulates like microbes into phagosomes, engage microtubules and motor proteins to move inwardly, contact and fuse with endosomes and lysosomes to degrade the enclosed particles (Harrison et al., 2003). Furthermore, extracellular cargo delivery through endocytosis forms vesicular endosomes that mature to late endosomes, and endolysosomes through microtubule-dependent fusion of late endosomes with lysosomes for cargo degradation (Huotari and Helenius, 2011; Storrie and Desjardins, 1996). Finally, microtubules regulate homotypic lysosome-lysosome contact with partial and/or complete fusion, where such fusion allows temporal exchange of luminal contents (Luzio et al., 2014; Storrie and Desjardins, 1996). Thus, microtubules serve as tracks upon which organelles gain mobility to find other target organelles and undergo heterotypic or homotypic fusion for cargo delivery or exchange, including lysosomes for such events.

Following microtubule dependent anchoring of lysosomes through motors, kinesin-1 and dynein, to target organelles, membrane tethering occurs to bring opposing membranes in close proximity for membrane fusion. Membrane tethering, for example may occur through HOPS (homotypic fusion and protein sorting) complex that interact with RILP, effector of Rab7-GTP, from opposing membranes of lysosomes and late endosomes (Bucci et al., 2000; Van Der Kant et al., 2015). Membrane tethering is followed by membrane fusion performed by SNARE proteins as discussed below. 


\subsubsection{SNARE proteins and membrane fusion}

Membrane fusion between organelles is essential for intracellular trafficking and exocytosis where cargo is released to the extracellular matrix (ECM). Membrane fusion is ultimately performed by SNAREs, including fusion with and between lysosomes. SNARE proteins have a 60-70 amino acid residue-long "SNARE motif” that assemble into an amphipathic $\alpha$-helix, a C-terminal transmembrane helix anchor, and an $\mathrm{N}$-terminal domain that modulates SNARE assembly and/or contact with vesicle docking/fusion machinery components. Membrane fusion occurs due to interaction between vesicle-associated v-SNAREs and tSNAREs found on target membranes. Such interaction is formed from four hydrophilic residues, where single arginine is contributed by R-SNARE (v-SNARE), and single glutamine contributed by each of Qa or Qb or Qc-SNARE (t-SNARE), thereby assembling SNARE proteins from different membranes to form trans-SNARE complex. (Baker and Hughson, 2016) (Fig. 3). Membrane fusion initiates from "zippering” starting at N-terminal ends of SNARE motifs and proceeding towards C-terminal transmembrane domains (CTD), thus pulling juxtaposed membranes together until fusion occurs (Mostafavi et al., 2017). Following zippering of the trans-SNARE complex towards the CTD, a fusion pore is formed between outer leaflets, followed by fusion of inner leaflets, which then elongates the pore and merges the two organelles, mixing content (Han et al., 2017). Following trans-SNARE complex assembly and membrane fusion, cis-SNARE complex is formed due to assembly of the SNAREs on the same membrane; the cis-SNARE complex is disassembled by the ATPase activity of Nethylmaleimide-sensitive fusion factor (NSF) and soluble NSF attachment proteins (SNAPs) (Baker and Hughson, 2016); v-SNAREs must then be recycled back to the donor organelle. The role of SNAREs in the context of lysosome fusion events will be discussed. 


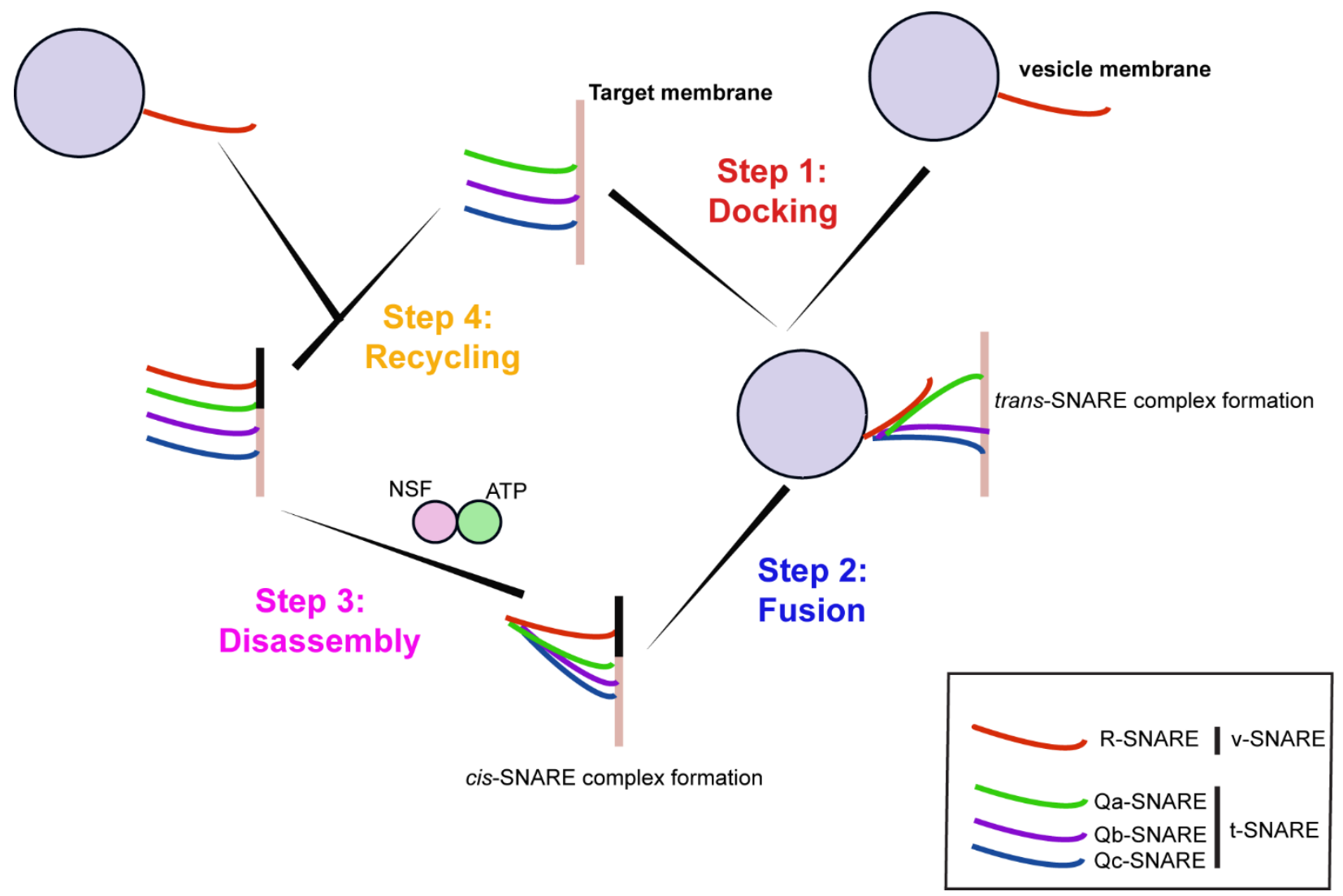

Figure 3: SNAREs in heterotypic membrane fusion. Membrane fusion between membranes of distinct organelle proceed with R-SNARE (v-SNARE) contributing arginine from vesicle membrane, and three Q-SNAREs (t-SNAREs), each contributing glutamine from target membrane. This followed by interaction between the R-SNARE and Q-SNAREs to form transSNARE complex (docking), membrane fusion and cargo exchange with assembly of the four SNARE proteins on single membrane as cis-SNARE complex (fusion), NSF-dependent ATPase activity to disassemble the cis-SNARE complex (disassembly), and recharging or resupply of SNAREs for additional rounds of membrane fusion (recycling). The v-SNARE recharged back 
on to the donor organelle membrane whereas t-SNAREs are available for docking and membrane fusion.

\subsubsection{SNAREs as dynamic mediators of lysosome membrane fusion}

Specific SNARE proteins function to tether membranes of lysosomes with late endosomes, phagosomes and autophagosomes for membrane fusion. For late endosomes and lysosomes, fusion is mediated by the Qa-SNARE syntaxin 7, the Qc-SNARE syntaxin 8, and the Qb-SNARE Vti1b on late endosomes and the R-SNARE VAMP7 on lysosomes (Pryor et al., 2004). In comparison, autophagosomes undergo fusion to form autophagosome-lysosome hybrid organelle (autolysosome) to degrade the cargo within autophagosome. Such maturation requires autophagosome transport to the perinuclear region, performed by the microtubule (-) end directed dynein-dynactin motor-adaptor complex (Mauvezin et al., 2016). Rab7 interact with the effector RILP for dynein dependent microtubule transport of endosomes, lysosomes and autophagosomes to the perinuclear region, followed by RILP and PLEKHM1 (pleckstrin homology and RUN domain-containing protein M1) recruitment of HOPS complex for membrane tethering. Thus, the HOPS complex may facilitate membrane tethering between autophagosomes and lysosome to bring membranes from these two opposing organelles together. Following membrane tethering, the SNARE STX17 on autophagosomes, interacts with HOPS complex and SNARE SNAP-29, and lysosome R-SNARE VAMP8 and these SNARE proteins facilitate membrane fusion for autolysosome formation (Diao et al., 2015; Jiang et al., 2014; Wijdeven et al., 2016). 
Phagocytosis involves internalization of foreign pathogens through plasma membrane remodelling and vesiculation to form phagosomes. Microtubule-dependent migration allows lysosomes to contact phagosomes (Harrison et al., 2003). During phagosome maturation into phagolysosomes, opposing membranes from phagosomes and lysosomes are tethered and merged through the action of SNAREs on opposing membranes. The R-SNARE VAMP7 and Qa-SNARE syntaxin 7 on lysosomes and Qbc-SNARE SNAP-23 on phagosomes co-ordinate to facilitate phagosome maturation into phagolysosomes (Sakurai et al., 2012).

Thus, SNAREs allow lysosome membrane fusion with endosomes, autophagosomes and phagosomes for maturation. Following such maturation, lysosomes must reform by separating from endolysosomes, autolysosomes and phagolysosomes through membrane remodelling to ensure continuous endosomal, phagosomal and autophagic flux as needed. As this requires membrane fission, I next discuss this process.

\subsubsection{Lysosome membrane fission}

Lysosomes can receive cargo through various possible dynamics of partial and/or complete lipid membrane homotypic or heterotypic fusion, followed by membrane splitting events. The dynamic equilibrium of constant fusion and fission cycles need to be maintained to achieve a steady state of lysosome number and size. Otherwise, processes that favor "fusion" while impeding "fission” may lead to fewer but enlarged lysosomes (Zou et al., 2015). To summarize, the dynamics of lysosome fission, can occur through budding/vesiculation (forming a vesicle), splitting (mid-section fission of lysosome) and/or tubulation (protrusion of lysosome membrane followed by budding to release vesicle). Additionally, lysosomes may undergo 
transient "kiss-and-run", temporary contact and bridge with pore formation between lysosome and another compartment (kiss) for luminal content exchange and membrane scission (run) to prevent complete coalescence between two compartments (Fig. 4) (Choy et al., 2018; Hipolito et al., 2018; Storrie and Desjardins, 1996). These dynamics of membrane fission may be part of a continuum. For example, during membrane tubulation, constriction during earlier phases of tubulation may lead to vesicle release and constriction during delayed phase of tubulation may lead to release of tubular intermediates. Vesiculation versus tubulation may be due to optimal regulation of ratio between surface area and luminal volume, where tubules have high surface area to volume ratio. For example, membrane tubulation are better for restricting luminal contents while enriching membrane components as a sorting mechanism (Freeman and Grinstein, 2018). I will elaborate on these mechanisms in greater detail after I introduce GTPases, phosphoinositides, and their role in membrane remodelling events. 

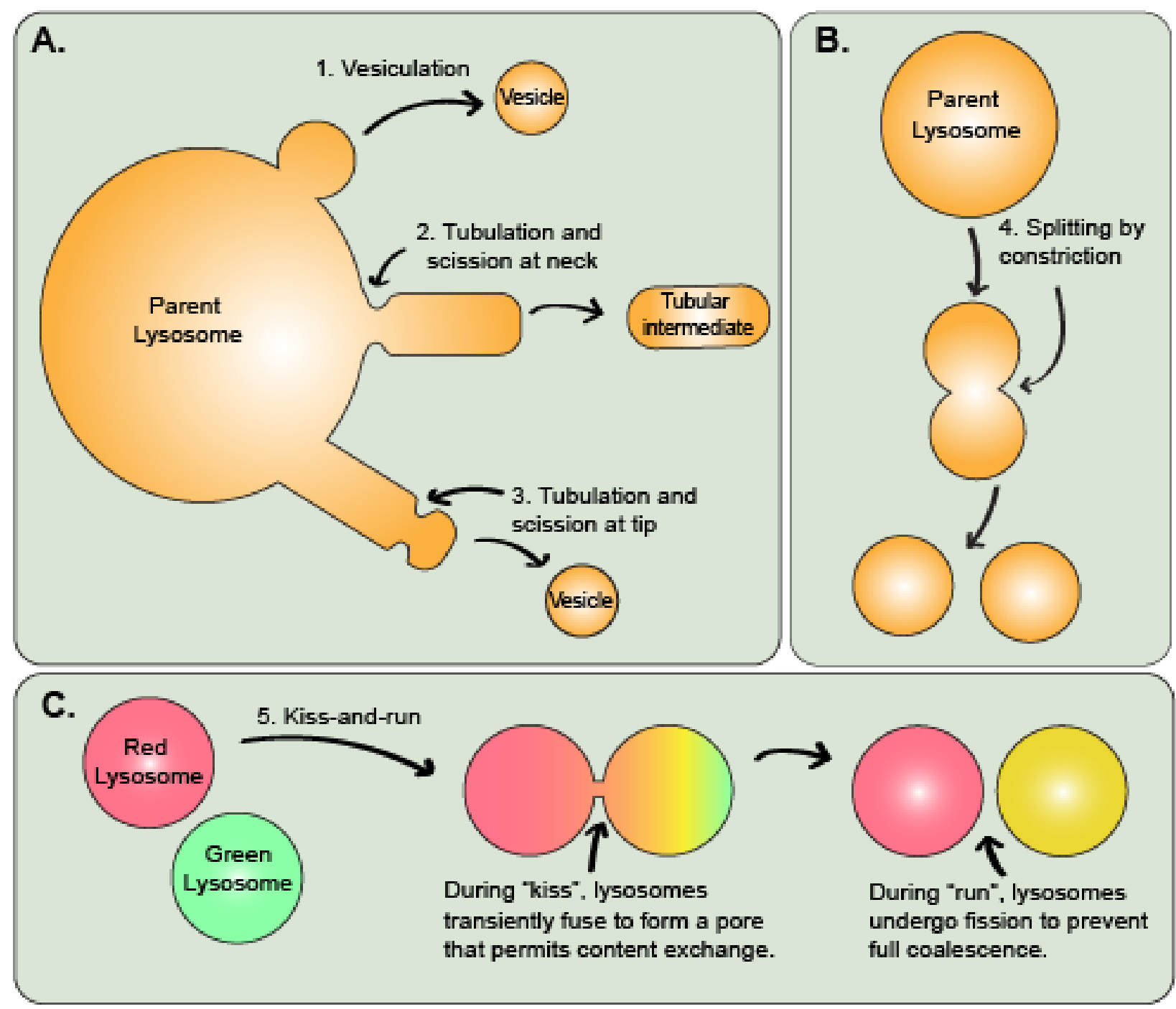

Figure 4: General dynamics of lysosome fission. A) Membrane vesiculation (budding) and tubulation. Donor lysosome membrane may undergo membrane deformation through coat protein that causes budding and scission to release vesicle (1). Nonetheless, the bud may extend for membrane tubulation that can be cut at the neck to release a tubular intermediate (2) or at tip to release vesicle (3) and/or tubules that are shorter. (B) Lysosome splitting may occur at midsection of tubular or vesicular membrane through constriction, from assistance of microtubule/actin and associated motor complexes (4). (C) Lysosome kiss-and-run (5): two lysosomes (green and red in the diagram), may contact transiently for restricted fusion where a 
bridge and pore temporarily forms between two lysosomes (kiss), followed by intraluminal content exchange that maybe bi-directional, or as shown unidirectional. This is followed by membrane scission (run) to prevent complete coalescence into a single enlarged lysosome.

The role of lysosome membrane remodelling during late stages of autophagy, endocytosis and phagocytosis following maturation and cargo degradation, in terms of membrane "reformation" and resolution will be discussed. Membrane phosphoinositides are important signalling lipids that co-ordinate with GTPases to define organelle identity and regulate membrane remodelling for vesicle formation and release from plasma membrane, endosomes and lysosomes. Therefore, understanding the identity and function of these GTPases and signaling lipids is important to relate mechanistic insight of lysosome membrane remodelling.

\subsection{GTPase and phosphoinositide regulation of membrane trafficking}

Organelles within a mammalian cell are compartmentalized due to lipid membranes that separate the lumen from the cytosol. How do proteins segregate to localize and signal for organelle specific functions? Such distinct protein localization and signalling is orchestrated through combination of unique membrane-bound GTPases and phosphoinositide signaling lipids, termed "coincidence detection" (Choy et al., 2017). Specific GTPases and phosphoinositides recruit effector proteins to the host membrane that effectively govern the properties and behaviour of the host membrane. Thus, different GTPases and phosphoinositides on different membranes elicits organelle identity and function. First, I will introduce GTPases regulation and function. 


\subsubsection{GTPase structure and function}

Ras superfamily of small monomeric GTPases are a class of proteins that shuttle between GDP-bound inactive state and GTP-bound active state and this cycle is regulated by GTPase activating proteins (GAPs) and guanine nucleotide exchange factors (GEFs), which mediate respectively GTP hydrolysis and loading. The Ras superfamily of GTPases consist of subfamily of GTPases, such as Ras, Rho, Rab and ADP ribosylation factor (Arf) GTPases, which are involved in signaling functions that can regulate cell division, differentiation, cytoskeletal organization, vesicle transport and nuclear assembly (Song et al., 2019). Ras GTPases contain two important domains, the first of which is a G box GDP/GTP-binding motif at the N-terminus: G1, GXXXXGKS/T; G2, T; G3, DXXGQ/H/T; G4, T/NKXD; and G5, C/SAK/L/T, which make up residues 5-166 that is $\sim 20 \mathrm{kDa}$ G domain. The second structural feature is C-terminal CAAX ( $\mathrm{C}=$ cysteine, $\mathrm{A}=$ aliphatic, $\mathrm{X}=$ any amino acid $)$ sequence for Rho or Ras GTPases. Alternatively, Rab GTPases display C-terminal CC, CXC, CCX, CCXX, CCXXX C-terminal motif. The C-terminal motif of Ras GTPases is recognized by farnesyl transferase or geranylgeranyltransferase I/II for the covalent addition of farnesyl or geranylgernanyl to cysteine, which facilitate membrane association and subcellular localization of these GTPases (Wennerberg et al., 2005). GTPases following organelle specific membrane localization, coordinate with membrane phosphoinositides to recruit effectors for directing various signaling functions. 


\subsubsection{Phosphoinositide structure and regulation}

Phosphoinositides (PtdInsP) are phospholipids found on eukaryotic cellular membranes and can be phosphorylated at various free hydroxyl positions in the polar myo-inositol head group to give rise to seven possible phosphoinositide species from the parent phosphatidylinositol (PtdIns) (Fig. 5). These species can be interconverted due to the action of lipid kinases, phosphatases and phospholipases, thus changing signaling functions as needed (Fig. 5). For example, PtdIns(4)P is converted to PtdIns(4,5) $\mathrm{P}_{2}$ through PtdIns(4)P 5-kinase, and conversely PtdIns(4,5) $\mathrm{P}_{2}$ is dephosphorylated to PtdIns(4)P through the Sac1 4-phosphatase (Nakanishi et al., 1995). Although it has been notably described of PtdInsP as seven distinct species depending on phosphorylation patterns on the inositol head group, they can be further classified depending on the acyl chain esterified to the glycerol backbone. Acylation of PtdInsP species occurs due to the acyltransferase LYCAT (lysocardiolipin acyltransferase). Depletion of LYCAT removes 18:0 stearic acid from the glycerol backbone and insert 16:0, 18:1 and 18:2. Although the most abundant acyl chain combination in mammalian cells is 1-stearic acid (18:0) and 2-arachidonic acid (20:4) for 38:4 combination, representing about 35-70\% of PtdInsP, there exist many acyl chain combinations with 14-22 carbon chains and up to six double bonds. Therefore, combination of acyl chain and inositol head group variations give rise to over 75 unique PtdInsP species (Choy et al., 2017).

PtdIns is synthesized within the endoplasmic reticulum (ER), which is transported to other membranes through vesicular transport or with the aid of cytosolic transfer proteins. In these membranes, PtdIns can be phosphorylated into one of the seven PtdInsP species. Typically, each PtdInsP species is thought to be found in a specific membrane, where it encodes organelle identity. However, this model is over simplistic since there is significant evidence showing that 
a single organelle can host more than one PtdInsP species (Fig. 6). (Choy et al., 2017; Sasaki et al., 2009; Traynor-Kaplan et al., 2017). Regardless of this, phosphoinositides perform signalling functions through interaction between their polar head groups with proteins of cytosol or cytosolic domains from membrane proteins. Such binding generally involves electrostatic interactions with the negative charged phosphate(s) on the polar inositol head group. These interactions can be strengthened by insertion of adjacent hydrophobic amino acids into the lipid bilayer. Typically, such interactions between proteins surfaces and phosphoinositide head group consist of cluster of basic amino acid residues from the protein and polar inositol head group. Examples of such interactions include the $\mathrm{N}$-terminus of lysosome associated calcium ion channel (TRPML1) that can interact with PtdIns(3,5) $\mathrm{P}_{2}$ or the pleckstrin homology (PH) domain of Phospholipase $\mathrm{C} \delta$ that can interact with phosphatidylinositol 4,5-bisphosphate $\left(\operatorname{Ptd} \operatorname{Ins}(4,5) \mathrm{P}_{2}\right)$ (Di Paolo and De Camilli, 2006; Dong et al., 2010). 


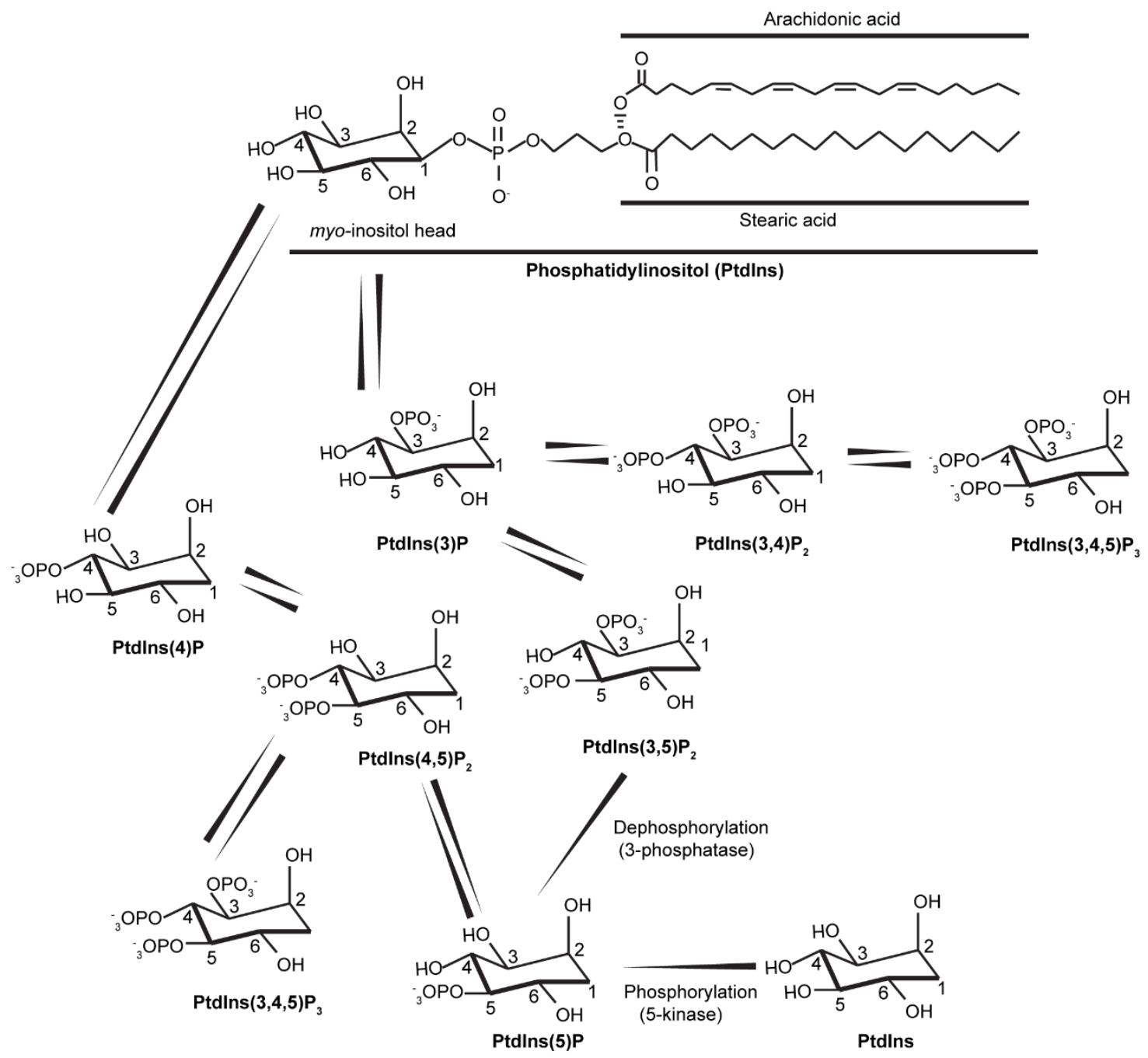

Figure 5: General structure of phosphatidylinositol. Structure of parental phosphatidylinositol (PtdIns) consists of glycerol backbone esterified to arachidonic acid (20:4) and stearic acid (18:0), and a phosphate that is attached to myo-inositol polar head group. The myo-inositol has free hydroxyl at D2-D6 positions, where D2-D5 can be phosphorylated or dephosphorylated in various combinations by lipid kinases and phosphatases to synthesize seven different phosphoinositide species: phosphatidylinositol 3-phosphate (PtdIns(3)P), phosphatidylinositol 4- 
phosphate (PtdIns(4)P), phosphatidylinositol 5-phosphate (PtdIns(5)P), phosphatidylinositol 3,4bisphosphate (PtdIns(3,4) $\left.\mathrm{P}_{2}\right)$, PtdIns(3,5) $\mathrm{P}_{2}$, PtdIns(4,5) $\mathrm{P}_{2}$, and phosphatidylinositol 3,4,5trisphosphate $\left(\operatorname{PtdIns}(3,4,5) \mathrm{P}_{3}\right)$.

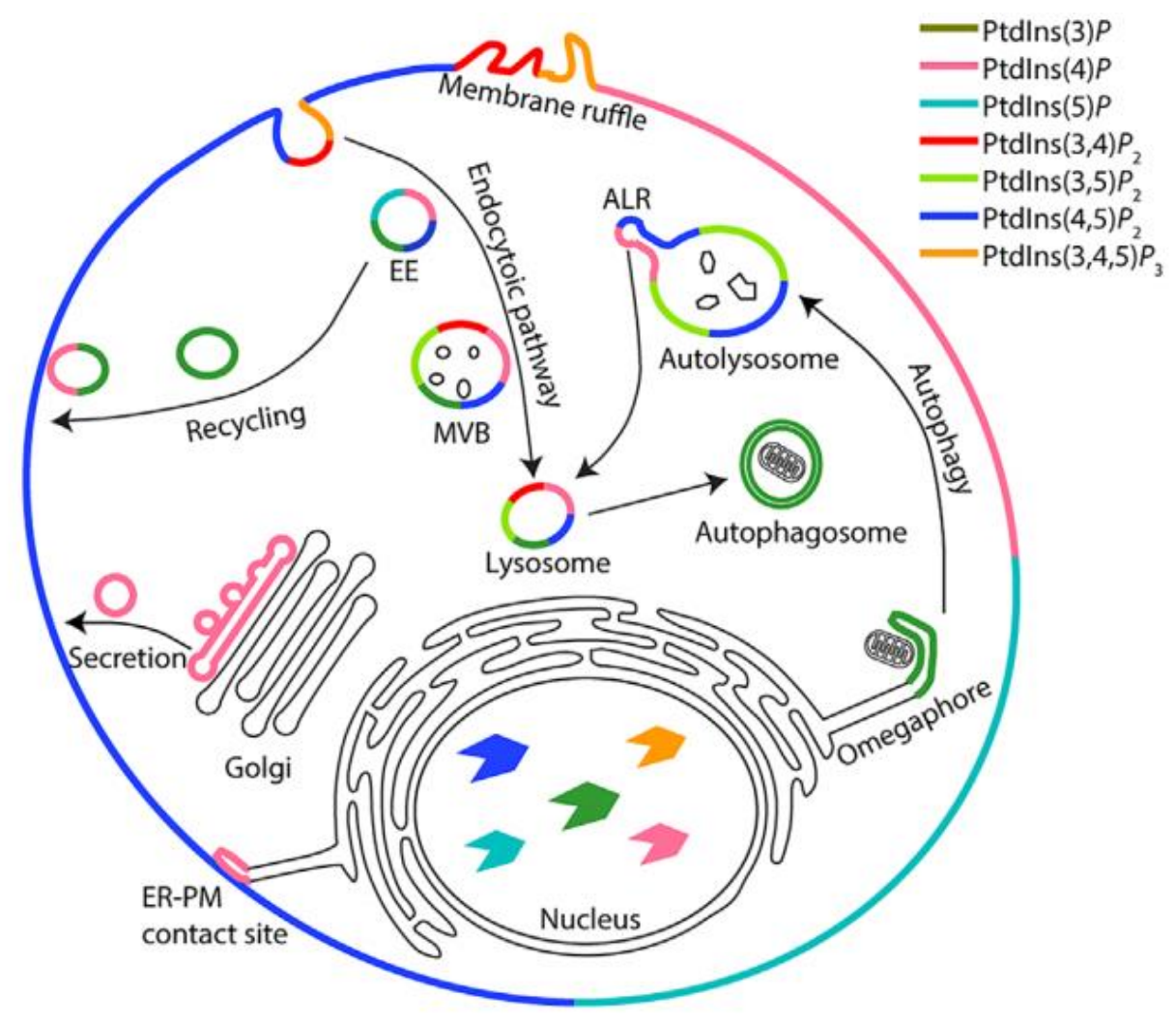

Figure 6: Lipid membranes display numerous phosphoinositide species. PtdInsP species are distributed on various organelle membranes, often with multiple species on single membrane and with similar species on different membranes (Choy et al., 2017).

PtdInsP species through "coincidence detection” co-ordinate with GTPases as regulators for organelle specific recruitment of protein effectors to perform various signalling functions as described below. 


\subsubsection{Co-ordination between GTPases and phosphoinositides for signaling}

PtdInsPs have numerous effectors and the same PtdInsP species can be found on different membranes. Therefore, there exist additional regulatory inputs for co-ordinating recruitment and function of specific effectors to PtdInsP species at the target membrane, and GTPases are a major regulatory input for such co-ordination. This leads to the concept of "coincidence detection" where combination of two or more regulators recruit effector protein to specific PtdInsP species at target membrane (Choy et al., 2017). Examples of such co-ordination between GTPases and PtdInsP species include interaction between early endosome antigen 1 (EEA1) with PtdIns(3)P through FYVE domain and Rab5-GTP on early endosomes to tether and induce EEA1-dependent homotypic fusion of endosomes (Lawe et al., 2002). Furthermore, FAPP1 and FAPP2 through PH domain have similar affinity for PtdIns(4)P and PtdIns(4,5) $\mathrm{P}_{2}$. However, these proteins exclusively localize to the Golgi membrane through interaction with Golgi associated PtdIns(4)P and Arf1-GTP, where they function in Golgi to plasma membrane transport (Godi et al., 2004; Mizuno-Yamasaki et al., 2010). Therefore, coincidence detection involving input signals can facilitate effector recruitment by phosphoinositides for signalling functions.

PtdInsP species regulate signaling and membrane remodelling as membrane lipids. I will discuss some of these signalling functions for maturation and membrane remodelling of the endosomal pathway. 


\subsubsection{Phosphoinositide signaling regulate endosomal maturation and membrane remodelling}

Phosphoinositide signaling on membranes regulate multiple steps along endocytosis that will be discussed, such as PtdIns(4,5) $\mathrm{P}_{2}$-dependent plasma membrane remodelling and vesiculation for internalizing extracellular nutrients, PtdIns(3)P-dependent cargo recognition and maturation of early endosomes to late endosomes, and $\operatorname{Ptd} \operatorname{Ins}(3,5) \mathrm{P}_{2}$-dependent reformation of terminal lysosomes from mature endolysosomes. During such maturation processes, PtdInsP dependent membrane remodelling and vesicle formation through splitting from donor membrane occurs from multiple compartments such as plasma membrane, endosomes and endolysosomes.

PtdIns(4,5) $\mathrm{P}_{2}$ perform important functions during clathrin mediate endocytosis (CME). CME is a critical process for internalization of nutrients, lipids, receptors and pathogens. The internalization process is initiated by formation of clathrin-coated pits $(\mathrm{CCP})$ within plasma membrane dome shaped invaginations, which with the assistance of various proteins causes initiation, growth and scission of the CCP. Clathrin recruitment to the plasma membrane occurs due to interaction of plasma membrane PtdIns(4,5) $\mathrm{P}_{2}$ with lysine residues of $\mu 2$-adaptin subunit of the adaptor complex AP-2, where AP-2 is required for clathrin assembly (Jackson et al., 2010). However, during this process, the actin cytoskeleton restructures to cause plasma membrane remodelling. The actin cytoskeleton around CCPs transform in shape from lateral patches around shallow pits during the early stages of endocytosis to a "comet tail" structure during the latter endocytic stages, which suggests that actin polymerization aids in propelling CCPs inwards towards the cytosol. F-BAR and double SH3 domain protein 2 (FCHSD2) activates actin polymerization during CME by interacting with Neural Wiskott-Aldrich Syndrome protein (N-WASP), which initiates actin nucleation for polymerization. FCHSD2 
additionally increases interaction between N-WASP and PtdIns $(4,5) \mathrm{P}_{2}$ where PtdIns $(4,5) \mathrm{P}_{2}$ can allosterically alter N-WASP to a active state for actin nucleation (Almeida-Souza et al., 2018; Rohde et al., 2002; Zigmond, 2000). Thus, PtdIns(4,5) $\mathrm{P}_{2}$ can initiate endocytosis by recruiting clathrin through AP-2, and also stimulate N-WASP dependent actin nucleation to propel clathrin containing vesicles inward towards the cytosol.

PtdIns(3)P at the early endosome membrane can facilitate early endosome maturation to late endosome by signaling through the ESCRT (Endosomal Sorting Complex required for transport) protein complexes, which involve cargo recognition, endosomal membrane remodelling and invagination followed by membrane scission to form intraluminal vesicles (ILVs). The ESCRT system comprises: ESCRT-0, ESCRT-I, ESCRT-II, ESCRT-III, and Vps (vacuolar protein sorting) 4 (Vps4) complex (Hurley, 2010). To initiate, Vps27 subunit of ESCRT-0 in yeast through FYVE domain interact with PtdIns(3)P at early endosomes. Two ubiquitin-binding (UIM) motif in Vps27 or single double-sided ubiquitin-binding (DUIM) in human Hrs (subunit of human ESCRT-0) bind ubiquitinated cargo due to ubiquitin being covalently transferred to cargo by E3 ubiquitin ligases from E2 ubiquitin-conjugating enzymes. Following ESCRT-0 assembly at early endosome membrane, ESCRT-I and ESCRT-II coassemble at endosome membrane followed by internal budding or invagination of the endosomal limiting membrane into the lumen. ESCRT-II recruits ESCRT-III through Vps25 subunit, and ESCRT-III performs membrane scission followed by disassembly of ESCRT-III complex from the endosomal membrane through ATPase action of Vps4. This leads to formation of ILVs for maturation of early endosomes to multivesicular endosomes (MVEs) or late endosomes (Hurley, 2010; Piper et al., 2014; Scott et al., 2005). In addition, PtdIns(3)P also is recognized by Phox (PX) domain of numerous sorting nexins, of which include SNX1, SNX2, SNX5 or SNX6, 
where these proteins form heterodimers. These SNX proteins possess C-terminal BAR domain capable of membrane remodelling and protrusion of stable tubules emanating from endosomes, and recruit Vps26-Vps29-Vps35 retromer cargo recognition complex to early endosome for endosome-TGN (trans-Golgi network) retrograde transport (Bissig and Gruenberg, 2013; Zhang et al., 2018). Therefore, PtdIns(3)P at early endosome membrane can signal through ESCRT complexes to induce membrane invagination and ILVs formation for maturation of early endosomes to late endosomes. In addition, PtdIns(3)P at early endosome membrane can recruit sorting nexins and retromer complex for membrane tubulation, and vesiculation for endosomeTGN cargo traffic.

$\operatorname{PtdIns}(3,5) \mathrm{P}_{2}$ is late endosome-lysosome (endolysosome) and lysosome membrane lipid that can induce membrane remodelling for vesiculation and release/reformation of terminal lysosomes from endolysosomes. Such vesiculation is postulated due to $\operatorname{Ptd} \operatorname{Ins}(3,5) \mathrm{P}_{2}$ dependent activation of lysosome associated mucolipin transient receptor potential channel (TRPML1) and subsequent intra-lysosomal calcium release towards the cytosol for calcineurin-dependent dynamin-1 activation, a GTPase involved in membrane scission, for membrane fission (Bissig et al., 2017; Zou et al., 2015).

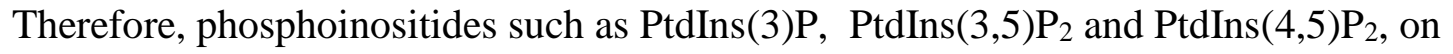
membranes of early endosomes, endolysosomes and plasma membrane regulate maturation of the endosomal pathway through membrane remodelling and vesicle formation. Of the different phosphoinositides involved in membrane remodelling, $\operatorname{PtdIns}(3,5) \mathrm{P}_{2}$ is the main focus of study for my dissertation and as such, the role of this lipid in lysosome membrane remodelling and signaling will be discussed. 


\subsubsection{PtdIns(3,5) $\mathrm{P}_{2}$ regulate lysosome signaling and membrane remodelling}

\subsubsection{Lipid kinase PIKfyve and PtdIns(3,5) $P_{2}$ synthesis}

PIKfyve in mammalian cells or Fab1 homologue in yeast is an evolutionarily conserved protein expressed from a single gene from yeast to plants to mammals. PIKfyve associate with membrane PtdIns(3)P and PtdIns to phosphorylate the D5 hydroxyl on the inositol head group to synthesize PtdIns(3,5)P 2 and PtdIns(5)P (Sbrissa et al., 2018; Stenmark et al., 2002; Zolov et al., 2012). PIKfyve has several conserved functional domains including, N-terminal FYVE domain that interact with PtdIns(3)P where the conserved sequence $\mathrm{R}(\mathrm{R} / \mathrm{K}) \mathrm{HHCR}$ is the site of interaction with PtdIns(3)P. The central region of PIKfyve contains CCT-like domain homologues to chaperonin-containing TCP-1 chaperonins. Adjacent to this domain is cysteine rich domain (CRD). The CCT-CRD domain is implicated in docking effectors Vac14 and Fig4 for regulating Fab1/PIKfyve function. The C-terminal domain of PIKfyve possess catalytic activity responsible for synthesizing $\operatorname{PtdIns}(3,5) \mathrm{P}_{2}$ and mutation in the predicted ATP-binding lysine at position 1831 depletes such catalytic functionality (Ho et al., 2012; Shisheva, 2008).

The functionality of PIKfyve depends upon complex formation with multiple protein partners, such as ArPIKfyve/Vac14 and Sac3/Fig4 in mammalian/yeast cells, and Vac7 and Atg18 in yeast. Vac14 consist of HEAT repeats and following dimerization serves as a scaffolding protein that nucleates the formation of a complex consisting of PIKfyve and interacting protein partners. Point mutation in HEAT repeat $4 \mathrm{~L} 156 \mathrm{R}$ leads to PtdIns $(3,5) \mathrm{P}_{2}$ depletion and Vac14 binding to PIKfyve, suggesting that HEAT repeat 4 mediates interaction between Vac14 and PIKfyve, where Vac14 and Fig4 docks at the CCT-CRD domain of PIKfyve. Fig4 is a 5-phosphatase, and converts $\operatorname{PtdIns}(3,5) \mathrm{P}_{2}$ to $\operatorname{PtdIns}(3) \mathrm{P}$. However, Fig4 is required to assemble into PIKfyve-Vac14-Fig4 complex to stimulate PIKfyve activity (Ho et al., 2012; Jin et 
al., 2008). Fig4 depletion deprives mammalian cells of $\operatorname{Ptd} \operatorname{Ins}(3,5) \mathrm{P}_{2}$, further suggesting a critical role of Fig4 in maintaining PtdIns(3,5) $\mathrm{P}_{2}$ synthesis (Zolov et al., 2012). Atg18, a PROPPIN which are $\beta$-propellers that bind phosphoinositides through conserved FRRG motif, interacts with PtdIns(3,5) $\mathrm{P}_{2}$ and is a negative regulator of PIKfyve since depletion of $\operatorname{Atg} 18$ results in increased PtdIns(3,5) $\mathrm{P}_{2}$ synthesis (Busse et al., 2015; Gopaldass et al., 2017). Vac7 found on yeast vacuoles, is a predicted single-pass transmembrane protein, and not conserved in higher eukaryotes. Furthermore, Vac7 interaction with Vac14 is not necessary for formation of active Fab1 (PIKfyve homologue in yeast) complex. Vac7 is a positive regulator of PIKfyve activity since depletion of Vac7 impair PtdIns(3,5) $\mathrm{P}_{2}$ synthesis (Duex et al., 2006; Ho et al., 2012). Thus, PIKfyve activation requires assembly into a complex consisting of Vac14, Fig4 and Atg18 for synthesis of PtdIns(3,5) $\mathrm{P}_{2}$ and PtdIns(5)P.

\subsubsection{PtdIns(3,5) $P_{2}$ signaling at the lysosome membrane and membrane remodelling}

$\operatorname{PtdIns}(3,5) \mathrm{P}_{2}$ at the endolysosome-lysosome membrane, despite being of minor abundance of about $0.05-0.1 \%$ of total PtdInsP, serves as a signaling lipid. For example, $\operatorname{PtdIns}(3,5) \mathrm{P}_{2}$ interacts with the $\mathrm{N}$-terminus of lysosome associated mucolipin transient receptor potential channel (TRPML1) to stimulate intralysosomal calcium release to regulate lysosome size. Depletion of either PtdIns(3,5) $\mathrm{P}_{2}$ or TRPML1 leads to lysosome enlargement (Dong et al., 2010b; Storrie and Desjardins, 1996; Zou et al., 2015). PtdIns(3,5) $\mathrm{P}_{2}$ interacts with cortactin at the filamentous actin (F-actin) binding domain, consisting of basic lysine residues. Cortactin localizes to actin branch to stimulate actin assembly through inducing WASP (Wiskott-Aldrich Syndrome) protein mediated actin nucleation. Competitive interaction of $\operatorname{PtdIns}(3,5) \mathrm{P}_{2}$ with cortactin prevent cortactin binding to F-actin, and thus inhibit actin nucleation at lysosomes to 
prevent lysosome-autophagosome maturation/fusion, suggesting role of $\operatorname{Ptd} \operatorname{Ins}(3,5) \mathrm{P}_{2}$ in regulating autophagy turnover through cortactin and actin assembly (Hasegawa et al., 2016;

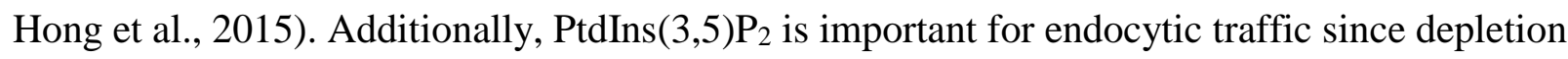
of this lipid impedes cargo traffic through the endosomes to lysosomes (Bissig et al., 2017). $\operatorname{PtdIns}(3,5) \mathrm{P}_{2}$ regulates phagosome maturation into phagolysosomes. Depletion of $\operatorname{PtdIns}(3,5) \mathrm{P}_{2}$ delays phagosomes docking onto lysosomes, thus decelerating phagosomes maturing into phagolysosomes and consequently preventing cargo degradation acquired through phagocytosis (Kim et al., 2014). PtdIns(3,5) $\mathrm{P}_{2}$ interacts with Atg18, which regulate vacuole/lysosome retrograde traffic to Golgi, suggesting role of $\operatorname{PtdIns}(3,5) \mathrm{P}_{2}$ in regulating lysosome/vacuole membrane recycling (Dove et al., 2004). Furthermore, PtdIns(3,5) $\mathrm{P}_{2}$ depletion accumulates proteins lipids and protein within the lumen of enlarged lysosomes, such as myelin basic protein (MBP) and myelin associated glycoprotein (MAG), suggestive of a role for PtdIns(3,5) $\mathrm{P}_{2}$ in regulating recycling of lysosome membrane and lumen contents for critical processes such as myelin formation on neuronal axon (Mironova et al., 2016). PIKfyve is implicated in retromer dependent endosome-to-Golgi retrograde traffic, suggesting role of $\operatorname{PtdIns}(3,5) \mathrm{P}_{2}$ in recycling proteins from endosomes to Golgi where PIKfyve depletion could lead to abnormal storage of contents within endocytic compartments (de Lartigue et al., 2009). Thus, $\operatorname{PtdIns}(3,5) \mathrm{P}_{2}$ is a important signaling lipid for regulating multiple stages of traffic at the lysosomes such as endocytic, autophagic, and phagocytic traffic to lysosomes, calcium release and lysosome size, lysosome membrane and lumen content recycling.

PtdIns(3,5) $\mathrm{P}_{2}$ is also suggested to be a important lipid for lysosome membrane remodelling since normal lysosomes with $\operatorname{PtdIns}(3,5) \mathrm{P}_{2}$ display membrane budding or 
tubulation. However, in the absence of PtdIns $(3,5) \mathrm{P}_{2}$, such membrane dynamic is remodelled to being absent of buds and tubules in enlarged lysosomes (Bissig et al., 2017).

PtdIns(3,5) $\mathrm{P}_{2}$ alters lysosome/vacuole morphology through membrane remodelling in response to stress. Yeast cells respond to hyperosmotic stress through a 16-20 fold increase in PtdIns(3,5) $\mathrm{P}_{2}$ levels with accommodating vacuolar fragmentation where vacuole number increase and vacuole size decrease. Such vacuole fragmentation presumably expels water to the cytosol to circumvent cytosolic water loss that is used to balance water content in the hypertonic environment (Bonangelino et al., 2002; Ho et al., 2012). The cyclin-dependent kinase Pho85 regulates Fab1/PIKfyve activity in yeast during hyperosmotic stress, where depletion of this protein abrogates PtdIns $(3,5) \mathrm{P}_{2}$ levels and compromise survival. The specificity of Pho85 for Fab1 was determined to be due to the interaction between Fab1 and the cyclin Pho80. Pho80Pho85 phosphorylates the kinase domain and the C-terminus of Fab1, and Vac7, which activate Fab1. The mammalian homologues of Pho80-Pho85, p35-CDK5, phosphorylates PIKfyve and regulates PtdIns $(3,5) \mathrm{P}_{2}$ synthesis in response to hyperosmotic stress, suggesting a conserved mechanism of Fab1/PIKfyve activation by Pho80-Pho85 across mammalian cell lines (Jin et al., 2017). I will further elaborate on PtdIns(3,5)P2 function in membrane fission in section 1.3.1.3 PtdIns(3,5) $\mathrm{P}_{2}$ regulate lysosome membrane reformation. 


\subsubsection{PtdIns(3,5) $P_{2}$ and pathophysiology}

PtdIns $(3,5) \mathrm{P}_{2}$ is an indispensable lipid that regulates traffic at the lysosome membrane. Thus, depletion of this signaling lipid leads to severe pathophysiological consequences. PtdIns(3,5) $\mathrm{P}_{2}$ is important for regulating multiple stages of neuronal integrity and loss of this lipid correlate with neurodegeneration in humans such as amyotropic lateral sclerosis (ALS) or charcot-marie-tooth type 4J (CMT4J) disease. Neuronal glutamate receptors such as $N$-methyl$D$-aspartate (NMDA) receptors through stimulation by glutamate causes calcium internalization through CaV1.2 channels. However, excessive calcium influx can lead to excitotoxic neuronal death. Prevention of such toxicity is regulated by PIKfyve, where stimulation of NMDA

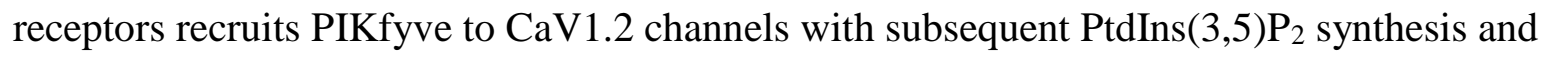
CaV1.2 targeting to lysosomes for degradation. Depletion of PIKfyve consequently results in excitotoxicity (Tsuruta et al., 2009). Additionally, as previously mentioned, $\operatorname{PtdIns}(3,5) \mathrm{P}_{2}$ depletion accumulates myelin components within enlarged lysosomes, preventing effective myelin formation on neuronal axons and preventing proper neuronal conductance of action potentials (Mironova et al., 2016). Amyloid precursor protein (APP) is a single spanning transmembrane protein, produced in the endoplasmic reticulum (ER) traversing between the Golgi, plasma membrane and endosome, that upon improper processing by beta and gamma secretase leads to amyloid-beta production, the principal peptide for which aggregation leads to neurodegenerative alzheimer disease. APP interacts with Vac14 of the PIKfyve complex and initiates PtdIns(3,5) $\mathrm{P}_{2}$ synthesis. Depletion of APP deprives PtdIns(3,5) $\mathrm{P}_{2}$ synthesis causing lysosome enlargement, thus raising the possibility that improper APP processing leading to APP depletion resulting in lysosome enlargement maybe one of the possibilities for 
neurodegeneration during Alzheimer's disease (Currinn et al., 2016). Thus, PtdIns(3,5) $\mathrm{P}_{2}$ may serve to regulate important trafficking processes that regulating neuronal integrity.

PtdIns(3,5) $\mathrm{P}_{2}$ may also regulate glucose homeostasis. Adipose tissue specific PIKfyve depletion in mice results in glucose intolerance and insulin resistance (Ikonomov et al., 2016). Additionally, the facilitative glucose transporter GLUT4 that upon stimulation by insulin translocate to the plasma membrane and import glucose inside adipose cells. Depleting PtdIns(3,5) $\mathrm{P}_{2}$ or PIKfyve prevents GLUT4 translocation to the plasma membrane and prevents

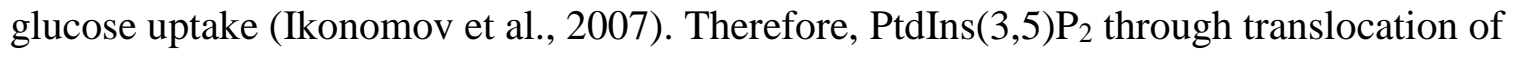
GLUT4 may regulate glucose uptake through insulin stimulation.

PtdIns $(3,5) \mathrm{P}_{2}$ is also implicated in inflammatory processes. PIKfyve depletion in mice lead to platelet dependent tissue inflammation where the platelets are defective for maturation, storage and lysosome enzyme release due to excessive storage and release of lysosome cargo proteins occur. Thus, inflammation from PIKfyve depletion occur due to abnormal lysosome storage and increased secretion of lysosomal contents from platelets (Min et al., 2014). However, PIKfyve inhibition by the selective antagonist apilimod has been reported to suppress production of IL-12/IL-23 in myeloid leukocytes, cytokines involved in inflammation and inducing psoriasis through infiltration of leukocytes into the dermis and epidermis (Wada et al., 2012). The difference in conflicting outcome for inflammation and PIKfyve inhibition may be due to platelets being selected for long-term genetic PIKfyve depletion for inflammation in mice, whereas oral administration of apilimod to human patients for 12 weeks might reflect a wider scale acute treatment for inflammation reduction.

PtdIns $(3,5) \mathrm{P}_{2}$ is implicated in a wide array of pathophysiological phenotypes ranging such as neurodegeneration, insulin resistance, inflammation. Furthermore, PIKfyve-null mice 
display embryogenic lethality and mice expressing partial PIKfyve exhibit defective organ development for multiple organs, such as organs of cardiopulmonary, nervous and hematopoietic systems (Ikonomov et al., 2011; Zolov et al., 2012). Thus, PtdIns(3,5) $\mathrm{P}_{2}$ is a critical signaling lipid for maintaining physiological integrity.

Since PtdIns $(3,5) \mathrm{P}_{2}$ regulates lysosome membrane remodelling, understanding additional regulators of lysosome membrane remodelling and reformation will give mechanistic insight into how multiple stages of membrane remodelling occur through the concerted action of these regulators.

\subsection{Lysosome membrane fission dynamics during autophagy, phagocytosis and endocytosis}

Lysosomes undergo cycles of fusion with autophagosomes (autolysosomes), phagosomes (phagolysosomes) and endosomes (endolysosomes), as a precursor for initiating processing of cargo during autophagy, phagocytosis and endocytosis. This is followed by reformation to retain a suite of lysosomes to maintain lysosome homeostasis and for lysosomes to be available for additional cycles of maturation into autolysosomes, phagolysosomes and endolysosomes (Bissig et al., 2017; Klionsky et al., 2014). Such reformation processes will be discussed in the context of microtubules/actin and motors, phosphoinositides, clathrin coat protein, and how all these factor into these membrane remodelling processes. 


\subsubsection{Endolysosome membrane remodelling and lysosome reformation}

Endocytosis, as previously discussed, involves uptake of extracellular nutrients through clathrin-dependent or -independent pathways, causing plasma membrane deformation and invagination to form vesicles that mature to early and late endosomes, and finally endolysosome from late endosome-lysosome fusion for cargo degradation (Elkin et al., 2016; Huber and Teis, 2016). Following endolysosome formation and cargo degradation, lysosomes reform from endolysosomes for additional cycles of maturation into endolysosomes, autolysosomes and/or phagolysosomes. Perhaps the best explanation for lysosome membrane "fission" and reformation is the normal "kiss-and-run" behaviour where lysosomes, late endosomes and endolysosomes undergo repeated transient contact followed by splitting through mechanisms yet to be completely understood. Research into mechanistic input for regulation of lysosome membrane reformation from endolysosomes suggest co-ordination of membrane phosphoinositides, coat proteins, cytoskeletal and associated motor protein complexes as dynamic regulators of membrane remodelling.

\subsubsection{Lysosome kiss and run}

Interactions between late endosomes and terminal lysosomes form endolysosomes that are hydrolase active and acidic (Bright et al., 1997; Bright et al., 2016). Using different pulse and chase periods for dextran rhodamine, which labelled lysosomes due to long pulse and chase, and dextran conjugated to Oregon green 488, which labelled late endosomes due to short pulse and chase, Bright et al. showed content mixing between "green" late endosomes and "red" lysosomes due to transient fusion (kiss). The red marker dissipated into the green compartment through a 
short tubule. This is followed by membrane budding and separation of the "red" lysosome for reformation into two separate compartments (Bright et al., 2005). Similarly, melanosomes through "kiss-and-run" interactions with lysosomes receive hydrolases necessary for maturation (Bissig et al., 2019). Lastly, using "Lyso-ATP”, which is a membrane permeable ATP and pH sensitive probe that accumulate within the acidic lysosome lumen, was used to demonstrate two lysosomes with different ATP levels undergo transient contact (kiss) leading to ATP transfer to the lysosome with less ATP, followed by separation (run) into two distinct lysosomes again (Jun et al., 2018). Lysosomes thus dynamically undergo constant "kiss-and-run" for transient bridge and pore formation (kiss) for luminal content exchange between two compartments, followed by membrane scission (run) to resolve into two separate compartments for preventing coalescence into single enlarged compartment (Fig. 4C).

\subsubsection{PtdIns(4)P co-ordinate with coat proteins for lysosome membrane reformation}

The lipid kinase PI4KIII $\beta$ and the phosphoinositide PtdIns(4)P on lysosome may regulate membrane remodelling. Depletion of PI4KIII $\beta$ increases lysosome tubule length and number, suggesting PtdIns(4)P assists in membrane scission and without PtdIns(4)P lysosome membrane tubulate since tubulated membrane cannot separate from lysosome. Interestingly, PI4K can physically interact with dynamin and in vitro studies demonstrate dynamin interaction with PtdIns(4)P, suggesting possible dynamin GTPase membrane scission mediated by PtdIns(4)P (Chang-Ileto et al., 2011; Sridhar et al., 2013).

The heterotetrameric protein complex AP-5 form a complex with spactacsin (SPG11) and spastizin (SPG15) to localize to lysosomes, and depletion of AP-5, SPG11 or SPG15 causes 
enlarged endolysosomes with aberrant storage of materials such as ganglioside lipid with neurons. Furthermore, SPG11 and SPG15 depletion reduces AP-5 levels, suggesting role of SPG11 and SPG15 in moderating AP-5 levels. SPG11 and SPG15 form a complex, and carry $\alpha-$ solenoids, domain found on coat proteins such as clathrin heavy chain and COPI subunits, suggesting possible membrane remodelling functions observed from coat proteins (Boutry et al., 2018; Chang et al., 2014; Hirst et al., 2013; Khundadze et al., 2013). AP-5 through SPG11 may regulate dynamin dependent lysosome membrane recycling and consequently lysosome reformation from endolysosomes (Boutry et al., 2018). Additionally, zebrafish spastizin interacts with dynamin and clathrin to remove VAMP4 to mature cortical granules (Kanagaraj et al., 2014). Moreover, AP-5 has been reported to regulate retrograde traffic from endolysosomes to the Golgi through retromer complex (Hirst et al., 2018). Thus, a plausible model maybe that PI4K and PI4P on lysosomes may nucleate assembly of spactacsin, spastizin and dynamin to promote membrane vesiculation. Indeed, PI4K physically interacts with AP-2 and spastizin (Chang et al., 2014; Sridhar et al., 2013).

\subsubsection{PtdIns $(3,5) P_{2}$ regulates lysosome membrane reformation}

PtdIns(3,5) $\mathrm{P}_{2}$ may regulate lysosome reformation from endolysosomes. Indeed, Fig4-/mice with depleted PtdIns(3,5) $\mathrm{P}_{2}$ display enlarged lysosomes due to defective PtdIns $(3,5) \mathrm{P}_{2}$ activation of the lysosome calcium channel TRPML1, thereby inhibiting calcium-dependent lysosome fission and depletion of membrane scission dynamin-1 GTPase (Zou et al., 2015). Additionally, PtdIns(3,5) $\mathrm{P}_{2}$ interacts with Atg18 to regulate vacuole to Golgi traffic (Dove et al., 2004). Atg18 is from the PROPPIN family of proteins, characterized by $\beta$-propellers that can bind phosphoinositides (Busse et al., 2015). Atg18 in yeast is yet the best understood PROPPIN 
in regulating vacuolar/lysosome fission. Depletion of Atg18 results in enlarged vacuoles that fail to fragment even in the presence of increased $\operatorname{PtdIns}(3,5) \mathrm{P}_{2}$ levels, and vacuole enlargement is rescued by tethering of Atg18 to vacuole membrane (Efe et al., 2007). Using unilamellar vesicles containing physiological levels of PtdIns(3)P and PtdIns(3,5) $\mathrm{P}_{2}$, Atg18 stimulated membrane tubulation and scission through a hydrophobic loop (CD loop) within blade 6 of the $\beta$-propeller that fold into an amphipathic $\alpha$-helix for insertion into the membrane that is $\operatorname{PtdIns}(3) \mathrm{P}$ and PtdIns(3,5) $\mathrm{P}_{2}$ dependent (Gopaldass et al., 2017). Although the role of yeast Atg18 was determined to regulate membrane remodelling and scission, it is as of yet not clear whether Atg18 counterparts in mammalian cells (WIPI-1 to -4) regulate lysosome membrane remodelling.

PtdIns $(3,5) \mathrm{P}_{2}$ may regulate lysosome membrane reformation through actin cytoskeleton. $\operatorname{PtdIns}(3,5) \mathrm{P}_{2}$ competes with actin for interacting with cortactin such that $\operatorname{PtdIns}(3,5) \mathrm{P}_{2}$ depletion lead to cortactin mediated actin nucleation and formation of actin aggregates on melanosomes and endosomes. This actin aggregate "trap" endosomes and possibly lysosomes to prevent proper endosome splitting or fission, leading to coalescence and enlargement (Bissig et al., 2019; Hasegawa et al., 2016). Thus, depletion of $\operatorname{PtdIns}(3,5) \mathrm{P}_{2}$ may accumulate actin on lysosomes to hinder "fission".

PtdIns(4)P and PtdIns(3,5) $\mathrm{P}_{2}$ thus are implicated in lysosome membrane remodelling and scission. Additionally, cytoskeletal structures such as microtubules and actin along with respective motor protein complexes are regulators that exert mechanical force and tension for lysosome membrane protrusion and separation as discussed below. 


\subsubsection{Microtubules and actin exert mechanical force for lysosome membrane fission}

Microtubules can exert mechanical force in response to stimuli for lysosome membrane remodelling and tubulation. PtdIns $(4,5) \mathrm{P}_{2}$ on autolysosome membrane recruits Kif5B in a clathrin-dependent manner to induce membrane tubulation to initiate lysosome reformation from autolysosomes (Du et al., 2016). Thus, microtubules and motor protein complexes, as well as upstream motor effectors regulate lysosome membrane remodelling to protrude membranes into tubulation, possibly as a pre-requisite for membrane scission from tubules by other proteins.

The actin cytoskeleton as well as associated motor complexes may play a role in lysosome membrane fission. Melanosomes, found within skin epidermal melanocytes and eye pigment cells, synthesize and store melanin pigment. Melanosomes are endosomal organelles that mature into pigmented organelles. Myosin 6 (Myo6), minus end filamentous actin (F-actin) motor, and the adaptor optineurin, along with actin nucleation machineries including Arp2/3 and WASH (Wiskott-Aldrich syndrome protein and SCAR homolog) complex on melanosomes participate in membrane scission through localization on neck of melanosome tubule, where inhibition of these components lead to membrane tubulation without scission (Ripoll et al., 2018). Although melanosomes are endosome-like organelles and not lysosome-like, this observation supports a model where actin assembly and nucleation machinery operate in lysosome membrane scission. To support this, LAMP1 structures in mouse embryonic fibroblasts enlarge and tubulate upon WASH depletion (Gomez et al., 2012).

Thus, cytoskeletal tracks such as microtubules and F-actin along with associated motor complexes may function to drive mechanical force to protrude lysosome membrane and facilitate membrane separation from endolysosomes. Additionally, membrane phosphoinositides and coat proteins participate through possible co-ordination with actin and microtubule cytoskeleton for 
lysosome membrane remodelling and fission (Fig. 7). The functional role of these regulators for lysosome reformation from autolysosomes and phagolysosomes will be discussed.

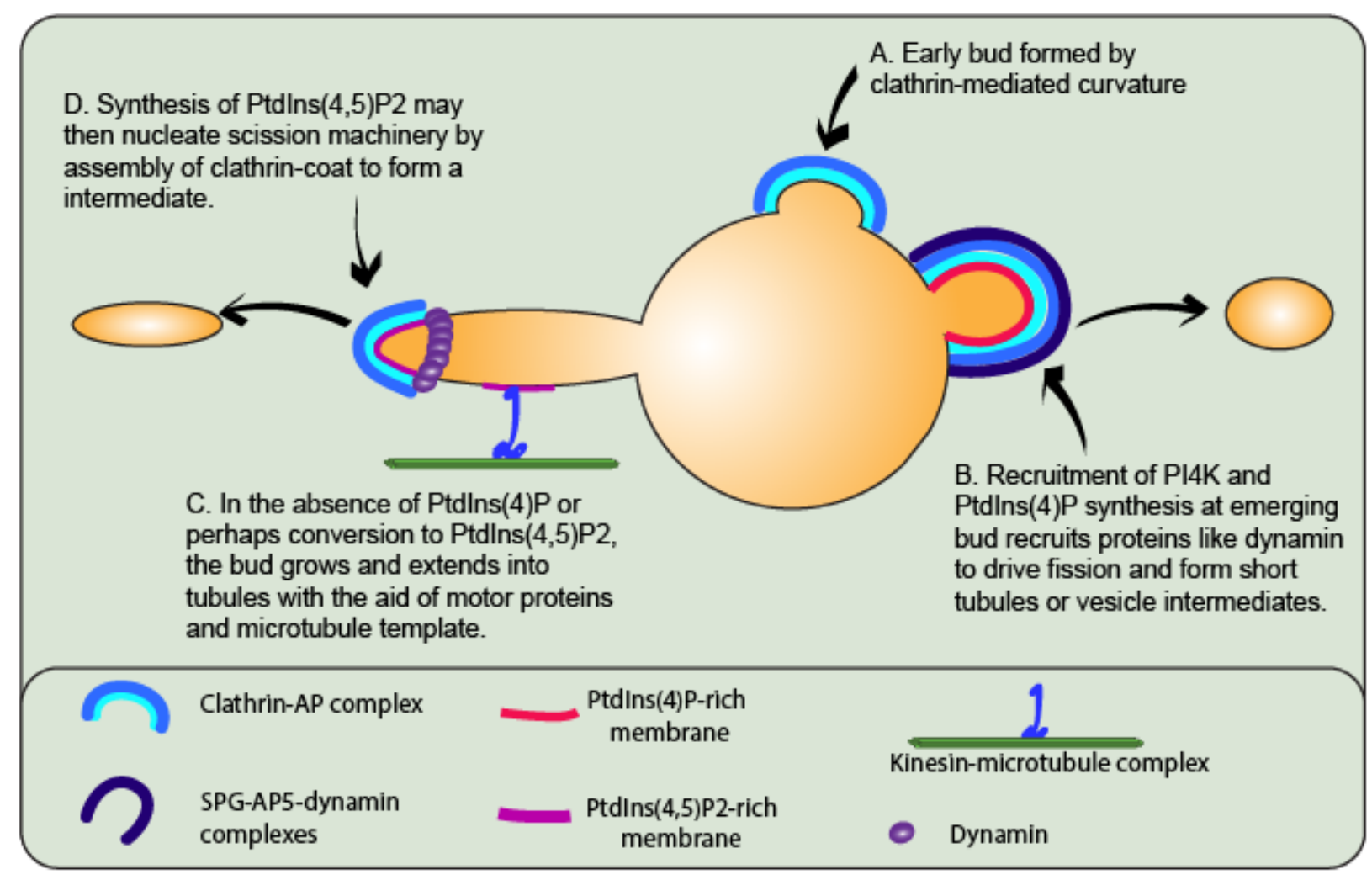

Figure 7: General mechanism of lysosome budding and tubulation. A) Budding through membrane curvature on the lysosome membrane maybe initiated by assembly of clathrin with associated adaptor protein (AP) complexes. B) PtdIns(4)P synthesis through phosphatidylinositol 4-kinase (PI4K) recruitment at the membrane bud may nucleate assembly of spactacsin, spastizin, AP-5, and/or dynamin, resulting in membrane scission release of bud as a vesicle, thus preventing bud protrusion into a tubule. C) Depletion of PtdIns(4)P and/or PtdIns(4,5) $\mathrm{P}_{2}$ conversion, may recruit microtubule motor kinesin on lysosome membrane, thus applying mechanical force for tubulation. D) The tubular extensions formed from (C) may increase 
localized PtdIns $(4,5) \mathrm{P}_{2}$ synthesis, which nucleate assembly of clathrin and/or dynamin to scission membrane at tip of tubule. This is a simplified speculative model absent of components that play a role in lysosome membrane reformation, such as PtdIns(3)P (Saffi and Botelho, 2019).

\subsubsection{Autolysosome membrane remodeling and lysosome reformation}

\subsubsection{Autophagosome initiation and maturation}

Autophagy is an intracellular lysosome dependent pathway that degrades cytosolic materials and organelles in response to stress conditions such as amino acid starvation, viral infection or unfolded protein response. Nutrient deprivation stimulated autophagy is nonselective and any part of the cytoplasm can be recycled back through this degradative pathway. However, autophagy can be selective in terms of selecting for cargoes such as protein aggregates, mitochondria, peroxisome, endoplasmic reticulum, signaling complex and pathogenic bacteria that are not needed, toxic, damaged or in surplus and thus require disposal (Johansen and Lamark, 2011; Khaminets et al., 2016; Mijaljica et al., 2012; Okamoto, 2014). Autophagy proceeds with inhibition of the serine/threonine protein kinase mammalian target of rapamycin complex 1 (mTORC1), which initiates a signaling cascade resulting in formation of double membrane bound autophagosome that engulfs a portion of the cytoplasmic material or cargo. The autophagosome fuses with late endosomes, forming amphisomes, followed by fusion with lysosomes, or direct fusion with lysosomes to form autolysosomes. Following autolysosome formation, the cytoplasmic and inner limiting membrane surrounding the cytoplasm are degraded by lysosome hydrolases and the degraded products are recycled back to cytoplasm where they can be reutilized for biosynthesis or producing energy (Eskelinen and Saftig, 2009). Following 
cargo degradation within autolysosomes, autolysosome reformation (ALR) is thus a process that couple nutrient recycling to lysosome reformation from autolysosomes (Rong et al., 2012).

\subsubsection{Membrane remodeling and vesiculation during ALR}

Following autolysosome formation and cargo degradation, lysosomes reform from autolysosomes through autophagic lysosome reformation (ALR). During ALR, tubules protrude from autolysosomes that are enriched for lysosome membrane components, followed by budding to release vesicles from these tubules. These vesicles, enriched for lysosome membrane components, are proto-lysosomes, and are initially non-acidic and void of luminal hydrolases. Eventually, they mature and gain acidic properties and luminal hydrolases. ALR is initiated through nutrient liberation and activation of mTORC1 (mammalian target of rapamycin) (Chen and Yu, 2017; Rong et al., 2012; Yu et al., 2010). ALR may initiate through recruitment of clathrin coat protein and its adaptor AP-2 to autolysosomes. PIP5K1 $\beta$, through interaction with PtdIns(4)P, synthesizes PtdIns(4,5) $\mathrm{P}_{2}$, which generates a feedback loop that additionally recruits AP-2 and clathrin on autolysosomes. Clathrin-AP2 complex on autolysosome membrane initiate membrane remodelling for budding and tubulation. This is followed by recruitment of PIP5K1 $\alpha$ on reforming tubules, which utilizes $\operatorname{PtdIns(4)P~to~initiate~a~second~round~of~} \operatorname{Ptd} \operatorname{Ins}(4,5) \mathrm{P}_{2}$ synthesis on tubules, followed by recruitment of Clathrin-AP2 on these tubules and subsequent vesiculation and release of proto-lysosomes (Rong et al., 2012). Kif5B, member of kinesin-1 family of microtubule motors that drive cargo transport towards the (+) end or GTP-cap of microtubules, also influences membrane tubulation during ALR. Kif5B through interaction with 
PtdIns $(4,5) \mathrm{P}_{2}$ on autolysosome membrane protrude the membrane into tubulation. However, such tubulation is clathrin-dependent since clathrin recruitment to autolysosome membranes initiate formation of PtdIns(4,5) $\mathrm{P}_{2}$ rich microdomains that recruit Kif5B for tubulation (Du et al., 2016; Randall et al., 2017). Dynamin 2, GTPase involved in membrane constriction and scission, is suggested to mediate vesiculation ALR from lipophagy, autophagy for processing of lipid droplets, since the absence of dynamin 2 promotes tubulation of autolysosomes and failure of lysosome reformation. Furthermore, $\operatorname{PtdIns}(4,5) \mathrm{P}_{2}$ may recruit dynamin 2 to autolysosomes (Rasineni et al., 2017; Yu et al., 2018). Therefore, the phosphoinositides PtdIns(4)P and PtdIns(4,5) $\mathrm{P}_{2}$, clathrin, AP-2 adaptor, dynamin, and Kif5B may act in concert to drive autolysosome membrane tubulation and budding for vesiculation and release of proto-lysosomes during ALR.

\subsubsection{Phagolysosome membrane remodelling and lysosome reformation}

\subsubsection{Phagocytosis initiation and maturation}

In mammalian cell, professional phagocytic cells such as macrophages, neutrophils, monocytes, dendritic cells, osteoclasts and eosinophils participate in eliminating foreign pathogenic microorganisms and apoptotic cells, and processing them for presentation to cells of the adaptive immune system (Flannagan et al., 2012; Gordon, 2016).

Phagocytes can recognize target particles through discrete receptors that can distinguish between targets and these receptors can be classified into non-opsonic or opsonic receptors. Examples of non-opsonic receptors include mannose receptors which bind mannan, CD-14 bind to lipopolysaccharide-binding proteins, SR-A bind to lipopolysaccharide (LPS) on some gram- 
negative bacteria and Dectin-1 bind to polysaccharide of some yeast cells. Opsonins include $\operatorname{IgG}$ antibodies, that interact with foreign particles, bridge the foreign particle to the phagocyte by interacting with Fc $\gamma$ receptors $(\mathrm{Fc} \gamma \mathrm{R})$ on phagocytes, where $\mathrm{Fc} \gamma \mathrm{R}$ recognize Fc portion of $\mathrm{IgG}$ molecules. Dendritic cells also house toll like receptors (TLRs) found at the plasma membrane, phagosomes and endocytic organelles, and can recognize structurally unrelated pathogenassociated molecular patterns (PAMPs) that evolutionarily conserved. For example, TLR3 recognize double stranded RNA, TLR9 recognize CpG DNA motifs, and TLR7/8 recognize single stranded RNA. Apoptotic cells release chemicals to the extracellular environment that serve as chemoattractant for phagocytic cells. Such chemicals include ATP, spingosine 1phosphate, and lysophosphatidylcholine. Additionally, apoptotic cells display on the plasma membrane phosphatidylserine (PS), which maybe recognized by receptors TIM-1, TIM-4, stabilin-2 and BAI-1 on phagocytes (Blander, 2008; Rosales and Uribe-Querol, 2017).

Following particle recognition, phagocyte receptors initiate signalling cascade that remodel cell membrane lipids and restructure actin cytoskeletion to facilitate membrane protrusion and internalization of the particle into phagosome. The phagosome proceeds towards maturation with endosomes and lysosomes to form phagolysosomes for particle degradation (Rosales and Uribe-Querol, 2017).

During phagosome maturation into phagolysosomes, opposing membranes from phagosomes and lysosomes are tethered and merged through the action of SNAREs on opposing membranes as previously discussed. Additionally, $\operatorname{Ptd} \operatorname{Ins}(3,5) \mathrm{P}_{2}$ controls phagosome maturation possibly by regulating calcium release through lysosome associated TRMPL1 (or mucolipin-1) channel, where depletion of the lipid or TRMPL1 leads to phagosomes docking onto lysosomes without being able to fuse. Intracellular calcium release was sufficient to rescue maturation 
(Dayam et al., 2015). Thus, PtdIns(3,5) $\mathrm{P}_{2}$ and TRPML1 stimulated calcium release, and SNAREs function to mature early phagosomes into phagolysosomes.

Following phagosome maturation into phagolysosomes and bacterial degradation, lysosomes must reform by separating from phagolysosomes to maintain lysosome number and size, as well as be available for maturation into phagolysosomes, endolysosomes or autolysosomes as needed.

\subsubsection{Phagolysosome membrane remodelling and lysosome reformation}

Entosis is a phagocytic process by which apoptotic cells are engulfed into entotic vacuoles within the cell, which mature by fusing with lysosomes followed by degradation. Overholtz and colleague, demonstrated mTORC1 activation by amino acids following degradation of entotic particle. Surprisingly, mTORC1 activation was required for entosome shrinkage and fission for lysosome reformation and redistribution of entotic contents to lysosomes (Krajcovic et al., 2013). Furthermore, the PtdIns(3,5) $\mathrm{P}_{2}$ lipid kinase PIKfyve, is involved in regulating size of mature entotic vacuoles merged with lysosomes, where PIKfyve and activation of the PtdIns $(3,5) \mathrm{P}_{2}$ effector TRPML1 lysosome associated calcium channel assists in lysosome reformation from mature entotic vacuoles. Furthermore, PIKfyve dependent lysosome reformation from mature entosomes is independent of mTORC1 (Krishna et al., 2016). Thus, PIKfyve and PtdIns(3,5) $\mathrm{P}_{2}$ function to stimulate lysosome reformation from mature entosomes in a process parallel to mTORC1. 
Lysosomes thus can reform from endolysosomes, autolysosomes and phagolysosomes through the concerted action of membrane phosphoinositides, coat proteins, cytoskeletal actin and microtubules, membrane scissoring dynamin GTPase, and mTORC1.

\subsection{Reactive oxygen species (ROS)}

There is evidence suggesting ROS regulating lysosome dynamics and interaction with autophagosomes, thus implicating a possible role of ROS interacting with endocytic compartments (Hamacher-Brady et al., 2011). Given this, I will introduce here what ROS are, how they are produced, how cells employ anti-oxidant defense systems to circumvent oxidative stress, the harmful and beneficial effects of ROS production.

\subsubsection{Types of ROS}

ROS are chemical species such as superoxide $\left(\mathrm{O}_{2}^{-}\right)$, produced through reduction of oxygen $\left(\mathrm{O}_{2}\right)$. Superoxide has one more electron than $\mathrm{O}_{2}$ on its outer orbital, and is thus more reactive due to inclination to receive another electron, thus being reduced in the process (Magder, 2006). Dismutation of superoxide by superoxide dismutase produce $\mathrm{H}_{2} \mathrm{O}_{2}$ which may be fully reduced to $\mathrm{H}_{2} \mathrm{O}$ or partially reduced to the highly oxidative hydroxyl radical (OH·) (Fig. 8). ROS may be produced enzymatically through NADPH (Nicotinamide adenine dinucleotide phosphate) oxidase and xanthine oxidase, or through the electron transport chain within the mitochondria 
(Sharma et al., 2012; Turrens, 2003). Depending on the context of how ROS are generated, different ROS species may have deleterious or beneficial outcomes for the cell.

\section{Superoxide anion:}

$$
\mathrm{NADPH}+2 \mathrm{O}_{2} \stackrel{\mathrm{NADPH} \text { oxidase }}{\longrightarrow} 2 \mathrm{O}_{2}^{-}+\mathrm{NADP}^{+}+\mathrm{H}^{+}
$$

\section{Hydrogen Peroxide:}

$$
2 \mathrm{O}_{2}^{-}+2 \mathrm{H}^{+} \stackrel{\mathrm{SOD}}{\longrightarrow} \mathrm{H}_{2} \mathrm{O}_{2}+\mathrm{O}_{2}
$$

\section{Hydroxyl Radical:}

$$
\mathrm{Fe}^{2+}+\mathrm{H}_{2} \mathrm{O}_{2} \longrightarrow \mathrm{Fe}^{3+}+\mathrm{OH}^{\cdot}+\mathrm{OH}^{\cdot}
$$

Figure 8: Mechanism of ROS production. Molecular oxygen $\left(\mathrm{O}_{2}\right)$ is reduced by NADPH oxidase for superoxide anion $\left(\mathrm{O}_{2}^{-}\right)$, superoxide anion is reduced by superoxide dismutase (SOD) through dismutation to produce $\mathrm{H}_{2} \mathrm{O}_{2}, \mathrm{H}_{2} \mathrm{O}_{2}$ is reduced through Fenton's reaction in presence of iron $\left(\mathrm{Fe}^{2+}\right)$ to produce hydroxyl radical $(\mathrm{OH})$ (Sharma et al., 2012; Yamazaki et al., 2011). 


\subsubsection{Synthesis of ROS}

ROS may be produced through mitochondrial dysfunction or through enzymes such as NADPH oxidase and xanthine oxidase. Much of intracellular ROS is produced within the mitochondria. The mitochondria possess an outer membrane, and inner membrane (IM) that are separated by the intermembrane space (IMS). The IM invaginates into to form cristae and encloses the matrix space. The IM also scaffolds the protein complexes of the electron transport chain. This places the respiratory chain between the acidic and oxidizing intermembrane space (IMS) due to presence of protons, and more the reducing and alkaline matrix (Dröse et al., 2014).

The mitochondrial respiratory chain consists of five protein complexes functioning to synthesize ATP from glucose through oxidative phosphorylation: complex I (NADH:ubiquinone oxidoreductase), complex II (succinate dehydrogenase), complex III/cytochrome $b_{1} c_{1}$ complex (ubiquinol-cytochrome c oxidoreductase), complex IV (cytochrome c oxidase), and complex V (ATP synthase). Complex I and complex II oxidizes the mitochondrial matrix NADH and succinate, respectively, acquired from the Krebs Cycle during aerobic respiration, and transfers electrons through the respiratory chain to complex IV to reduce molecular oxygen $\left(\mathrm{O}_{2}\right)$ to water. During this electron transfer process, redox energy transfer protons from the mitochondrial matrix to the IMS to build a proton motive force across the IM, which is used to produce ATP at the matrix from ADP and inorganic phosphate by complex V (ATP synthase). Inhibition of complex I or III can lead to ROS generation, where electron build-up can cause electron leaks or single electrons to react with oxygen to produce superoxide anion $\left(\mathrm{O}_{2}{ }^{-}\right)$. Superoxide can then produce $\mathrm{H}_{2} \mathrm{O}_{2}$ and hydroxyl radicals (Lemarie and Grimm, 2011; Sharma et al., 2009).

NADPH oxidase from the NOX family of enzymes are multiunit membrane associated enzymes that use NADPH as an electron donor to transport electrons across membranes for one- 
electron reduction of $\mathrm{O}_{2}$ to $\mathrm{O}_{2}^{-}$(superoxide). Superoxide can then react to form other ROS such as $\mathrm{H}_{2} \mathrm{O}_{2}$ and hydroxyl radicals. There are seven possible NOX isoforms that have been identified: NOX1, NOX2, NOX3, NOX4, NOX5, dual oxidases (DUOX1 and DUOX2). NOX2 is extensively studied as being associated with phagosomes in phagocytic cells that display oxidative burst for pathogen killing for host defense (André-Lévigne et al., 2017; Brown and Griendling, 2009). NOX2 is composed of a catalytic core transmembrane complex of gp91 phox and $\mathrm{p} 22^{\text {phox }}$, forming a heterodimeric flavocytochrome $b_{558}$. The regulatory subunits of NOX2 are cytosolic, consisting of $\mathrm{p} 67^{\text {phox }}, \mathrm{p} 47^{\text {phox }}, \mathrm{p} 40^{\text {phox }}$ and the Rac GTPase. NOX2 requires assembly of the six subunits into a membrane bound functional complex to become active. This assembly

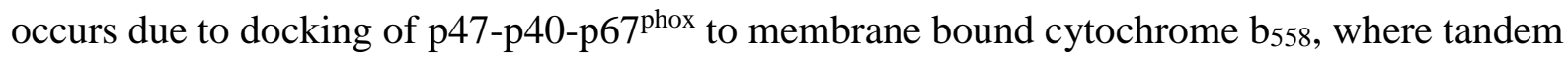
SH3 domain of $\mathrm{p} 47^{\text {phox }}$ interact with C-terminus of p22 ${ }^{\text {phox }}$. Following independent membrane translocation of $\mathrm{p} 67^{\text {phox }}$ and Rac-GTP, they form the p67-Rac complex with cytochrome $b_{558}$ to complete the assembly and mediate electron transfer (Groemping and Rittinger, 2005). Thus, assembled NOX2 participates in electron transfer for ROS stimulation and bacterial killing.

Xanthine oxidase, localized within the cytosol, is produced during inflammation when xanthine dehydrogenase (XDH) undergoes oxidation at cysteine residues (535 and 992) and/or proteolysis to form $\mathrm{XO}$. XO is part of the purine degradation pathway, by oxidizing hypoxanthine to xanthine and subsequent xanthine oxidation to uric acid. However, during these reactions, $\mathrm{O}_{2}$ is used as a redox partner, which undergoes one electron and two electron reduction reactions to produce superoxide and $\mathrm{H}_{2} \mathrm{O}_{2}$, respectively (Cantu-Medellin and Kelley, 2013; Ichikawa et al., 1992; Sakuma et al., 2015). ROS exert beneficial or harmful impact on cell depending on the context which they are used for. Additionally, ROS participate in localized 
signalling to regulate organelle specific function. Therefore, ROS can locally regulate lysosome function.

\subsubsection{Harmful effects of ROS}

ROS are very damaging to cellular components such as lipids, proteins and DNA. In addition, they may induce apoptosis in response to ischemia and stimulate inflammation to damage tissues. Hydroxyl radical $(\mathrm{OH} \cdot)$ is very reactive towards lipids. $\mathrm{OH} \cdot$ can oxidize membrane lipids into lipid radicals that upon reaction with oxygen produce hydroperoxide radicals (LOO). This radical can interact with another lipid to form lipid peroxides that then form a reactive and destructive chain. This can lead to membrane damage as well as formation of byproducts such as 4-hydroxy-2-nonal and $\beta$-unsaturated aldehydes that may inactivate proteins through reaction with cysteine, lysine or histidine residues (Forman et al., 2009). Hydroxyl radicals are also damaging towards DNA nucleotides by reacting with thymine through a series of oxidation and reduction reactions to produce nucleotide radicals that can interact with adjacent nucleotides forming DNA-crosslinks. Additional, hydroxyl radicals may undergo a series of redox reactions with guanine to form a radical cation that interacts with lysine to form DNAprotein crosslinks. Cytosine may also undergo redox reactions with hydroxyl radicals leading to oxidation products that are unstable and sensitive to deamination to produce uracil-analog products, or isomerization into a mixture of products (Cadet and Wagner, 2013). Additionally, ROS elevation is correlated with DNA strand breaks (Bibov et al., 2018).

On the other hand, superoxide and $\mathrm{H}_{2} \mathrm{O}_{2}$ can damage proteins by reacting with thiols of cysteine residues. However, superoxide is less stable compared to $\mathrm{H}_{2} \mathrm{O}_{2}$ and undergo dismutation 
to $\mathrm{H}_{2} \mathrm{O}_{2}$ before reaction with biothiols. Additionally, superoxide cannot cross biological membrane due to negative charge. Contrarily, due to the stability of $\mathrm{H}_{2} \mathrm{O}_{2}$ compared to superoxide and ability to diffuse across biological membranes, $\mathrm{H}_{2} \mathrm{O}_{2}$ can oxidize thiol residues from cysteine progressively to produce disulfide bonds (-SSR), sulfenic acid (-SOH), and irreversible sulfenic acid $\left(\mathrm{SO}_{2} \mathrm{H}\right)$ and sulfonic acid $\left(\mathrm{SO}_{3} \mathrm{H}\right)$, which can lead to protein damage and inactivation (Brown and Griendling, 2009; Schieber and Chandel, 2014). Therefore, ROS as chemical species possess oxidative potential to damage components that constitute cellular integrity, such as proteins, lipids and DNA.

Overt ROS accumulation can also trigger apoptosis and loss of mitochondrial integrity. Mitochondrial dysfunction may occur in response to ischemia, where reduction in blood flow to the myocardium can induce oxidative stress and mitochondrial damage. Damage to the electron transport chain complex 1 during ischemia elevates ROS production (Lesnefsky et al., 2017). Mitochondria normally display an extended, tubular interconnected network, but they fragment into punctate mitochondria during ROS-mediated oxidative stress. Such mitochondrial fragmentation correlates with a reduction in the mitochondrial membrane potential causing increased membrane permeability, impaired respiration and oxidative phosphorylation, and an increase in mitochondrial ROS production, all indicators of mitochondrial dysfunction. This results in increased association of pro-apoptotic Bax with mitochondrial OM, where Bax and its homologue Bcl-2 homologues antagonist (Bak) oligomerize to permeabilize mitochondrial outer membrane. Permeabilization is followed by release of pro-apoptotic factors such as cytochrome c and apoptosis-inducing factor that result in caspase-dependent or -independent cell death (Ježek et al., 2018). Given that ROS can exert harmful outcome for cell physiology, there must exist defensive intracellular anti-oxidant systems to circumvent excessive ROS production. 


\subsubsection{Anti-oxidant systems to circumvent ROS production}

Elevated ROS are harmful to the cell, damaging cellular components and even causing cell death. Therefore, cells have evolved anti-oxidant systems as defense against elevated ROS. Below, I introduce three four key anti-ROS systems deployed by cells: Superoxide dismutase, catalase, and glutathione.

Superoxide dismutase (SOD) converts $\mathrm{O}_{2}^{-}$to $\mathrm{H}_{2} \mathrm{O}_{2}$ and molecular oxygen $\left(\mathrm{O}_{2}\right)$. SOD is a metalloenzyme and exists as different isoforms; mitochondria SOD utilizes manganese (MnSOD) while the cytosol and peroxisomes express SOD that employs copper/zinc (Cu/Zn-SOD).

The general conversion reaction is $\mathrm{O}_{2}^{-}+\mathrm{O}_{2}^{-}+2 \mathrm{H}^{+} \rightarrow \mathrm{O}_{2}+\mathrm{H}_{2} \mathrm{O}_{2}$ (Ighodaro and Akinloye, 2018; Mccords and Fridovich, 1969). In addition, excess $\mathrm{H}_{2} \mathrm{O}_{2}$ can be toxic to cells and is thus degraded by catalase. Catalase, located within peroxisomes and mitochondria, utilize heme as a cofactor to degrade two molecules of $\mathrm{H}_{2} \mathrm{O}_{2}$ to two molecules of $\mathrm{H}_{2} \mathrm{O}$ and $\mathrm{O}_{2}$ (Marengo et al., 2016; Salvi et al., 2007; Schrader and Fahimi, 2008).

Glutathione (GSH), located within the mitochondria and cytosol, is a tripeptide of $\gamma$-Lglutamyl-L-cysteinyl-glycine, that utilize sulfhydryl (-SH) group for reduction and conjugation reactions for removal of peroxides. Glutathione peroxidase uses GSH as a substrate to reduce $\mathrm{H}_{2} \mathrm{O}_{2}$ to $\mathrm{H}_{2} \mathrm{O}$ and GSSG (two glutathione oxidized at sulfhydryl (-SH) groups to form disulfide bond). Since high levels of GSSG is toxic to cells, GSSG is reduced back to GSH by glutathione reductase using NADPH as electron donor (Lubos et al., 2011). 


\subsubsection{Beneficial impact of ROS}

Although overproduction of ROS impart detrimental outcome for cell physiology, controlled levels of physiologically relevant ROS production can drive cell proliferation and induce immune response against foreign pathogens. ROS can regulate growth factor signaling. Growth factor signaling through epidermal growth factor (EGF) and platelet-derived growth factor (PDGF) occurs through autophosphorylation of specific tyrosine residues on the cytoplasmic tail of receptor tyrosine kinases (RTKs) on the plasma membrane to recruit proteins such as PI3K-Akt involved in cell proliferation. The RTKs, epidermal growth factor receptor (EGFR) and platelet-derived growth factor receptor (PDGFR), are sensitive to inactivation by protein phosphatases PTP1B and SHP-2 respectively. EGF and PDGF stimulate ROS production, and $\mathrm{H}_{2} \mathrm{O}_{2}$ can oxidize sulfenic moieties on cysteine residues of PTP1B and SHP-2, and PTEN, protein phosphatase for inactivating PI3K, to inactivate these phosphatases and promote sustained growth factor signaling. Thus, a positive feedback loop is established where growth factor signaling stimulate ROS production to prevent RTK inactivation, and sustain continual growth factor signaling. Although ROS can promote growth factor signaling by inactivating RTKs, cancer cells generate increased ROS to elevate cellular proliferation while also increasing anti-oxidant activity to prevent ROS mediated cellular damage (Hanahan and Weinberg, 2011; Lee et al., 1998; Park et al., 2018; Schieber and Chandel, 2014). Therefore, although ROS mediated growth factor signaling can be beneficial for the cell, but if stimulated excessively can promote cancer cell growth.

NADPH oxidase 2 (NOX2) uses $\mathrm{O}_{2}$ as electron acceptor to produce superoxide and $\mathrm{H}_{2} \mathrm{O}_{2}$. NOX2 on phagosome membrane of phagocytic cells assemble to kill bacteria (Groemping and Rittinger, 2005). Additionally, neutrophils house azurophilic granules containing 
myeloperoxidase (MPO), that upon immune stimulation utilizes $\mathrm{H}_{2} \mathrm{O}_{2}$ from NADPH oxidase activity, to reduce chloride ions to the highly oxidative hypochlorous acid ( $\mathrm{HOCl})$. $\mathrm{HOCl}$ can oxidize proteins, lipids and DNA, which as discussed may be detrimental to these components (Metzler et al., 2011; Odobasic et al., 2016). Therefore, ROS serve as important line of defense against bacterial pathogens. During immune response, ROS can stimulate inflammation. Specifically, ROS can activate NF- $\mathrm{B}$ transcription factor for release of pro-inflammatory cytokines and inflammasome activation, thus contributing to inflammation (Forrester et al., 2018). Inflammation is a beneficial immune response to defend against foreign pathogens through dilation of blood vessels and increasing blood vessel permeability, which serve to recruit leukocytes at sites of infection for release of enzymes and chemicals for pathogen destruction. However, excessive stimulation of inflammation lead to inflammatory diseases such as arthritis and Crohn's disease (Mittal et al., 2014). Therefore, although ROS may beneficially induce inflammation as part of immune response, overstimulation may be harmful.

\subsubsection{ROS regulate lysosome function and dynamics}

ROS may regulate lysosome dynamics through localized signalling to lysosomes. ROS generating NOX2 is reported to be on lysosomes (Li et al., 2012). ROS production cause clustering of lysosomes and impedes autophagosome turnover, due to impaired autophagosomelysosome fusion (Hamacher-Brady et al., 2011; Zheng et al., 2016). Mitochondria are proximally located to lysosomes and mitochondrial ROS stimulation activate TRPML1 ion channel on lysosome membranes to release calcium. This causes calcium to activate the phosphatase calcineurin to dephosphorylate transcription factor EB (TFEB) for activation. TFEB is then released from the lysosome membrane and translocate to nucleus to regulate expression of genes 
from CLEAR (co-ordinated lysosome expression and regulation) motif that upscale lysosome and autophagosome biogenesis, thus increasing the cellular pool of autophagosomes and lysosomes for autophagic degradation of dysfunctional mitochondria (Möller et al., 2019; Zhang et al., 2016). Although unrelated to lysosome dynamics, ROS stimulation has been reported to stimulate mitochondrial fragmentation through "fission" through dynamin-related protein 1 (Drp1) (Hung et al., 2018; Zhao et al., 2017). Thus, localized ROS stimulation regulate lysosome biogenesis and fusion as well as possible fission dynamics.

ROS synthesized locally to and from lysosomes may regulate actin and microtubule structure, cytoskeletal tracks for regulating lysosome fusion-fission dynamics as previously discussed, and thus control lysosome dynamics. Monomeric actin contains five cysteine and sixteen methionine residues, which contain sulfhydryl (-SH) for cysteine or S-C bonds for methionine susceptible to oxidation by ROS. Impacts of oxidation of - $\mathrm{SH}$ groups have been discussed, and oxidation of methionine leads to methionine sulfoxide (MetO) that alter the three dimensional structure of proteins to expose hydrophobic regions that are normally buried to perturb protein functions. Cytosolic exposed residues such as Cys374, Met176, Met190, and Met 355 are susceptible to oxidation. An oxidative environment inhibits actin polymerization while suppressing ROS promote actin polymerization, supporting the role of ROS in regulating actin structure through (Dalle-Donne et al., 2003; Kim et al., 2014; Sakai et al., 2012; Wilson and González-Billault, 2015). However, ROS stimulation is needed for actin-dependent membrane protrusions in Ptk1 marsupial kidney epithelial cells, suggestive role of ROS at physiological levels to positively regulate actin structure (Taulet et al., 2012). $\alpha$ - and $\beta$ - tubulin, monomers of microtubules, have 12 and 8 cysteine residues respectively sensitive to oxidation. Similar to actin, oxidative environment suppresses tubulin polymerization, which is reversible by the 
glutathione/glutathione reductase system (Landino et al., 2004; Pocasap et al., 2018; Wilson and González-Billault, 2015). Therefore, ROS can impair microtubules and actin structurally, and thus possibly impede the cytoskeletal tracks for regulating lysosome fusion-fission dynamics as a novel phenotype for regulating cargo traffic to and from lysosomes, which is the focus of my dissertation. 


\subsection{Research objective}

Since PIKfyve inhibition and PtdIns(3,5) $\mathrm{P}_{2}$ depletion enlarges lysosomes, my overall thesis objectives were i) to better understand how lysosome enlargement proceeds in PIKfyve inhibited cells and ii) to better understand molecular processes that govern lysosome size. To achieve these objectives, I had two main hypotheses.

\subsubsection{Hypothesis I}

Lysosomes enlarge during PIKfyve inhibition from one and/or both of two possibilities (Fig. 9).

Hypothesis Ii: Each individual lysosome grows in size through increased biosynthesis. In this scenario, lysosome number is predicted not to change but cells gain in total lysosome volume.

Hypothesis Iii: Individual lysosomes coalesce to form larger but few lysosomes. In this scenario, there should be no increase in total lysosome volume. 


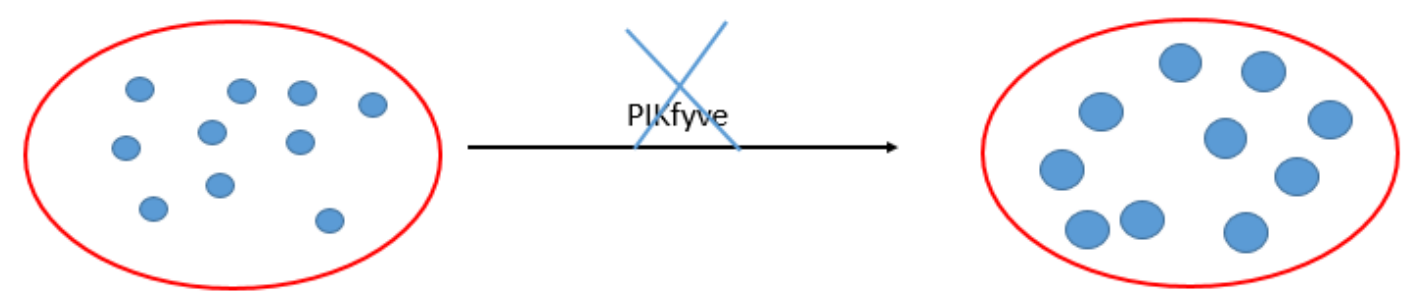

Model 1: Lysosomes get bigger in size while staying same in number
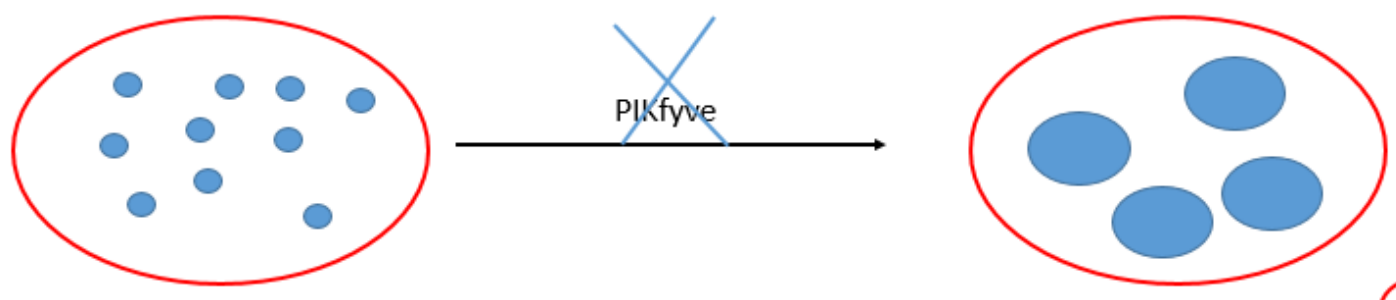

Model 2: Lysosomes fuse to get bigger in size while becoming fewer in number

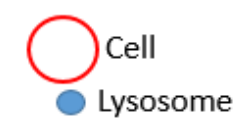

Figure 9: Possible mechanisms for lysosome enlargement during PtdIns(3,5) $P_{2}$ depletion.

Lysosomes become enlarged during PtdIns(3,5) $\mathrm{P}_{2}$ depletion through one of two possible dynamics. Model 1: Each individual lysosome becomes enlarged, while lysosome number remains unchanged, increasing total lysosome volume per cell. Model 2: Lysosomes fuse together to increase in size, decreasing in number but maintaining total lysosome volume per cell.

\subsubsection{Objectives}

1. Use quantitative image analysis for measuring individual lysosome volume, lysosome number and total lysosome volume per cell, and score impact of acute pharmacological and genetic PtdIns(3,5) $\mathrm{P}_{2}$ depletion on lysosome morphology. 
2. Use quantitative image analysis to investigate reversibility of lysosome enlargement upon PIKfyve reactivation

3. To understand whether lysosome fission and fusion cycling is affected by $\operatorname{Ptd} \operatorname{Ins}(3,5) \mathrm{P}_{2}$ depletion.

4. To understand whether microtubules play a role in lysosome enlargement during PIKfyve inhibition.

Upon completion of experimental observations to complete above mentioned objectives, we concluded that lysosome enlargement during acute and genetic PtdIns(3,5) $\mathrm{P}_{2}$ depletion occurs due to increase in individual lysosome volume, while decreasing lysosome number, while total lysosome volume remained unchanged (Fig. 9, Model 2). This enlargement is reversible upon PIKfyve reactivation and PtdIns(3,5) $\mathrm{P}_{2}$ synthesis. Additionally, lysosomes undergo dynamic continuous transient partial and/or complete fusion and fission cycles, and impaired "splitting" during PIKfyve inhibition contributes to lysosome "coalescence" for enlargement. Furthermore, microtubules and associated motor protein complexes regulate lysosome dynamics to affect enlargement during PIKfyve inhibition and lysosome "shrinkage" during PIKfyve reactivation. During our experimental observations, we accidentally happened upon potent abatement of lysosome enlargement during acute PIKfyve inhibition due to microscopy laser-based ROS stimulation. This led the directions for our second research objective and hypothesis: ROS stimulation affects lysosome dynamics and regulates "fusion/fission" during acute PIKfyve inhibition and reactivation. 


\subsubsection{Hypothesis II}

During our study to test hypothesis I, we noticed that intense light during imaging prevent lysosome enlargement during PIKfyve inhibition. We thus postulated that ROS can arrest lysosome enlargement during acute PIKfyve inhibition and PtdIns(3,5) $\mathrm{P}_{2}$ depletion. We further hypothesized that this inhibition might proceed as follows.

Hypothesis: ROS stimulation prevents lysosome enlargement during acute PIKfyve inhibition by impeding lysosome "coalescence" AND/OR accelerating lysosome "fragmentation/fission"

\subsubsection{Objective}

1. Use quantitative image analysis for measuring individual lysosome volume, lysosome number and total lysosome volume per cell, and understanding how these parameters respond to ROS stimulation during acute pharmacological PIKfyve inhibition and PIKfyve reactivation

2. To understand how ROS stimulation affect lysosome movement through evaluating: lysosome speed, track length, displacement, microtubule and actin structure which are cytoskeletal tracks affecting lysosome motility

3. To understand whether ROS affects lysosome "fragmentation" through increasing $\operatorname{PtdIns}(3,5) \mathrm{P}_{2}$ synthesis and/or assembly of fission machinery on lysosomes: clathrin and dynamin

4. To understand whether ROS promote clearance of actin mass on lysosomes, that otherwise hinder fission, to promote fission 
We observed that ROS stimulation through multiple pharmacological manipulations $\left(\mathrm{H}_{2} \mathrm{O}_{2}\right.$, rotenone, 1-chloro-2,4-dinitrobenzene (CDNB), monochlorobimane (MCB)) was antagonistic to lysosome coalescence during acute PIKfyve inactivation and accelerated lysosome "fragmentation" during PIKfyve reactivation. ROS stimulation does not increase PtdIns(3,5) $\mathrm{P}_{2}$ synthesis to increase lysosome fragmentation. Depending on the type of ROS and/or site of ROS synthesis, lysosome dynamics affected distinctly. $\mathrm{H}_{2} \mathrm{O}_{2}$ arrested lysosome motility and stably extended the fibrillary structure of microtubules although such stability may not contribute to arrested lysosome enlargement, suggesting decreased lysosome mobility to impair coalescence. Additionally, $\mathrm{H}_{2} \mathrm{O}_{2}$ increased lysosome associated clathrin and dynamin, suggesting assembly of lysosome fission machinery, although such recruitment was not accompanied by $\mathrm{H}_{2} \mathrm{O}_{2}$ mediated lysosome fragmentation during PIKfyve reactivation. However, other ROS agonists tested (rotenone, CDNB and MCB), through superoxide generation, depolymerized microtubule structure without affecting lysosome movement. Furthermore, ROS produced by these agonists promoted clearance of actin patches from lysosomes, suggesting ROS mediated removal of actin mass on lysosomes, that otherwise hinder fission, to promote fission. 


\section{Chapter 2: Materials and Methods}




\subsection{Materials and Methods}

\section{Cell culture, plasmids, and transfection}

RAW 264.7 macrophages and HeLa cells were grown in Dulbecco's Modified Eagle Medium (DMEM; Wisent, St Bruno, QC) supplemented with 5\% heat-inactivated fetal bovine serum (FBS; Wisent). ARPE-1 (RPE) cells stably expressing clathrin heavy chain-eGFP were grown in DMEM/F12 medium (Gibco) supplemented with 10\% FBS (Aguet et al., 2013). Mouse embryonic fibroblasts (MEFs) grown in 15\% FBS supplemented Roswell Park Memorial Institution (RPMI) media (RPMI-1640: Gibco, Burlington, ON). All cells were grown at 5\% $\mathrm{CO}_{2}$ and $37^{\circ} \mathrm{C}$ and routinely checked for contamination. FuGene HD (Promega, Madison, WI) was used for transient transfections following manufacturer's instructions with a ratio of 3:1 FuGene HD transfection reagent $(\mu \mathrm{l})$ to DNA $(\mu \mathrm{g})$. The transfection mixture was replaced with fresh complete medium 4-5 h post-transfection and cells were used $24 \mathrm{~h}$ following transfection. RAW cells were transfected with plasmids expressing Rab7-RILPC33-GFP (RILPC33-GFP) or wildtype Arl8b-GFP (Arl8bWT-GFP) or Kif5B ${ }^{\text {DN }}$-RFP or p50-dynamitin-GFP, which were previously characterized (Cantalupo et al., 2001; Echeverri et al., 1996; Hofmann and Munro, 2006; Silver and Harrison, 2011).

\section{Pharmacological treatment of cells}

Apilimod (Toronto Research Chemicals, Toronto, ON) was used at $20 \mathrm{nM}$ for $40 \mathrm{~min}$, unless otherwise indicated, to deplete cellular PtdIns(3,5) $\mathrm{P}_{2} \cdot \mathrm{H}_{2} \mathrm{O}_{2}$ (Bio Basic, Markham, ON) was used as indicated. Rotenone, 1-chloro-2,4,-dinitrobenzene (CDNB; Sigma-Aldrich, Oakville, ON) and 
monocholorobimane (MCB; Sigma-Aldrich, Oakville, $\mathrm{ON}$ ) were used as indicated to generate ROS by respectively inhibiting mitochondrial respiratory chain complex I, thioredoxin reductase and depleting glutathione. Bovine liver catalase (Sigma-Aldrich) and N-acetyl-L-cysteine (NAC) (Bio Basic) were used as anti-oxidants. Paclitaxel and nocadozole (both from Sigma-Aldrich) were used at 1 or $10 \mu \mathrm{M}$ and 5 or $10 \mu \mathrm{M}$ to stabilize and depolymerize microtubules, respectively. Ciliobrevin D used at $100 \mu \mathrm{M}$ to inhibit dynein activity (EMD Millipore, Toronto, ON). Latrunculin A (Abcam, Toronto, ON) and cytochalasin D (EMD Millipore, Toronto, ON) used at $10 \mu \mathrm{M}$ and $5 \mu \mathrm{M}$ respectively to depolymerize actin. Ikarugamycin (Sigma-Aldrich) and dyngo-4A (Cambridge, MA, USA) used to inhibit clathrin and dynamin respectively.

\section{Lysosome labelling}

Lysosomes were labelled by incubating cells with $200 \mu \mathrm{g} / \mathrm{mL} \mathrm{Alexa}^{546}$-conjugated dextran or Alexa $^{488}$-conjugated dextran (Thermo Fisher Scientific, Mississauga, ON) or with $2.5 \mathrm{mg} / \mathrm{mL}$ Lucifer yellow (Thermo Fisher Scientific, Mississauga, ON) for $2 \mathrm{~h}$ in complete media at $37^{\circ} \mathrm{C}$ in $5 \% \mathrm{CO}_{2}$. Cells washed with phosphate-buffered saline (PBS) and resupplied with complete cell-specific media for $1 \mathrm{~h}$ to chase the fluid-phase marker to lysosomes before pharmacological manipulation and live-cell imaging. We note that we use "lysosomes" to represent a potential mixture of late endosomes, lysosomes and endolysosomes (Bright et al., 1997; Choy et al., 2018). 


\section{Live- and fixed-cell spinning disc confocal microscopy}

Imaging evaluating effect of $\operatorname{PtdIns}(3,5) \mathrm{P}_{2}$ depletion and synthesis on lysosome volume and number, and involvement of microtubules and associated motor protein complexes during these processes, performed with Olympus IX81 inverted microscope equipped with a Hamamatsu C9100-13 EMCCD camera and a 60X 1.35 N.A. objective and controlled with Volocity 6.3.0 (PerkinElmer, Bolton, ON). Microscopy and imaging assessing impact of ROS stimulation on lysosome number and volume during acute PIKfyve inhibition and reactivation done with Quorum DisKovery spinning disc confocal microscope system equipped with Leica DMi8 microscope connected to iXON 897 EMCCD camera, controlled by Quorum Wave FX powered by MetaMorph software, using 63x 1.4 NA oil-immersion objective (Quorum Technologies, Guelph, ON). Live-cell imaging was performed using environmental chamber set to $5 \% \mathrm{CO}_{2}$ and $37^{\circ} \mathrm{C}$ in complete cell-specific medium. Standard excitation and emission filter sets and lasers were used for all fluorophores. RAW and HeLa cells, unless otherwise indicated, were imaged as z-projections of 45-55 z-planes with $0.3 \mu \mathrm{m}$ distance between each plane, or 20-30 z-planes with $0.3 \mu \mathrm{m}$ distance between each plane for RPE cells, as acquired by spinning disc confocal microscopy.

\section{Sweptfield confocal microscopy live cell imaging}

RAW cells incubated 2 hours with $200 \mu \mathrm{g} / \mathrm{mL}$ of Alexa ${ }^{555}$-conjugated dextran (Thermo Fisher Scientific) in complete DMEM media, followed by wash with PBS and replenishment with fresh complete media for 90 min for labelling lysosomes. Live cell imaging performed with Nikon Ti inverted microscope, equipped with 100x 1.49 N.A. objective, and swept-field slit 
scanning confocal scan head (Prairie Technologies, Sioux Falls, SD), controlled by Nikon NIS Elements software. Images acquired with Photometrics Prime 95B back illuminated sCMOS camera, while high speed triggered acquisition was controlled by Mad City Labs Piezo Z stage controller and National Instruments DAQ card. Sample image volumes acquired at $30 \mathrm{~s}$ intervals over $30 \mathrm{~min}$, at $100 \mathrm{~ms}$ per $\mathrm{z}$-slice, prior to addition of $20 \mathrm{nM}$ apilimod and then image volumes acquired $2 \mathrm{~h}$ in presence of apilimod.

\section{Intracellular ROS imaging}

For determining intracellular ROS production, we incubated RAW 264.7 macrophages with 5 $\mu \mathrm{M}$ of the cell-permeable redox sensitive dye, CellROX Green (Thermo Fisher Scientific), for 30 min at $37{ }^{\circ} \mathrm{C}$ with $5 \% \mathrm{CO}_{2}$ in the dark during treatment with various ROS producing agents. Cells were washed twice with PBS followed by replenishment with complete media and imaging.

\section{Immunofluorescence}

Following experimentation, cells were fixed for 15 min with 4\% (v/v) paraformaldehyde in PBS, permeabilized for 10 min with $0.1 \%$ Triton X-100 (v/v) in PBS, and then blocked with 3\% BSA (v/v) in PBS. Subsequently, cells were incubated with mouse monoclonal antibody against $\alpha-$ tubulin (1:200; Sigma-Aldrich), followed by incubation with donkey Dylight-conjugated polyclonal antibody against mouse IgG (1:1000; Bethyl), and samples were then mounted in Dako mounting media for subsequent imaging. Alternatively, lysosomes were labelled with Alexa ${ }^{488}$-conjugated dextran, and cells fixed for 15 min with 4\% (v/v) paraformaldehyde in PBS, 
permeabilized for 10 min with $10 \mu \mathrm{g} / \mathrm{ml}$ digitonin (promega, Madison, WI) in PBS, blocked with 3\% BSA (v/v) in PBS, and stained for actin with phalloidin (Thermo Fisher Scientific, Mississauga, ON).

\section{Image analysis}

To determine lysosome number, individual lysosome volume and total cellular lysosome volume, we used Volocity (Volocity 6.3.0) particle detection and volumetric tools. Z-stack images were imported into Volocity and a signal threshold was applied at 2x the average cytosolic fluorescence intensity. Particles were defined as being greater than $0.3 \mu \mathrm{m}^{3}$ for inclusion into the analysis, and if necessary, a watershed function was applied to split lysosome aggregates caused by thresholding. Regions of interest were drawn surrounding individual cells for cell-to-cell analysis. Lysosome splitting frequency, speed, track length, and displacement was assessed using Imaris (BitPlane, Concord, MA) with 'ImarisTrackLineage' module, where "splitting" was defined as frequency of events when a single particle gave rise to two particles. This is an approximation for fission and does not exclude the possibility that two overlapping or adjacent particles separated and were counted as "fission" event.

To determine the level of membrane-bound RILP-C33 and Arl8b, we estimated the membrane-bound to cytosolic ratio of fluorescently-tagged proteins. Using ImageJ, lines that were 3-pixel wide by 20-40-pixel long were assigned to areas of transfected cells using a predetermined grid to avoid bias but excluding the nucleus. Plot profiles were then obtained, exported into an Excel spreadsheet, values were arranged according to fluorescence intensity, and the ratio calculated for highest 10 pixels over lowest 10 pixels along the length of the line 
$\left(\mathrm{F}_{\mathrm{H}} / \mathrm{F}_{\mathrm{L}}\right.$ fluorescence ratio); the expectation is that values approximate to 1 represent low membrane signal due to mostly cytosolic signal, while ratio values greater than 1 represent signal that localizes to punctate structures relative to cytosol (Chintaluri et al., 2018).

For determination of clathrin-GFP on lysosomes, RPE cells stably expressing clathrin heavy chain-eGFP were loaded with Alexa ${ }^{546}$-conjugated dextran and treated with apilimod, followed by imaging with spinning disc confocal microscope. Image analysis was performed using ImageJ by thresholding Alexa ${ }^{546}$-conjugated dextran signal and generating a mask, which was then applied to the green (clathrin) channel to determine the GFP fluorescence intensity on regions marked by dextran signal. Regions of interest within the cytosol and the extracellular space were drawn to respectively obtain mean cytosolic fluorescence intensity and background. These values were then used to calculate the ratio of lysosome-to-cytosol clathrin-eGFP. To determine intracellular CellROX Green fluorescence or MCB-GSH adduct fluorescence emissions, images were imported onto Volocity (Volocity 6.3.0), regions of interest drawn around cell, and mean fluorescence intensity per cell recorded. Image contrast enhancement was performed with Adobe Photoshop CS (Adobe Systems, San Jose, CA) or ImageJ without changing relative signals and applied after quantification. Adobe Illustrator CS (Adobe Systems) was used for constructing figures.

\section{Lysosome fractionation}

RAW 264.7 cells were grown and used according to manufacturer's instructions to obtain membrane fractions by differential sedimentation ultracentrifugation using a density gradient (Lysosome Isolation Kit, Sigma-Aldrich, LYSISO1). Briefly, cells were lysed and homogenates 
centrifuged $1,000 \mathrm{xg}$ for $10 \mathrm{~min}$ at $4{ }^{\circ} \mathrm{C}$ to separate unbroken cells and debris from cytoplasmic membranes. The supernatant was further centrifuged at $20,000 \mathrm{xg}$ for $20 \mathrm{~min}$ at $4{ }^{\circ} \mathrm{C}$ to pellet lysosomes and other organelles. The pellet was reconstituted with optiprep density gradient medium $(60 \%(\mathrm{w} / \mathrm{v})$ solution of iodixanol in water and sucrose) and loaded onto of a step-wise sucrose gradient as described by the manufacturer and subjected to ultracentrifugation at 150,000 $\mathrm{x} g$ for $4 \mathrm{~h}$ at $4{ }^{\circ} \mathrm{C}$ using SW50.1 rotor (Beckman Coulter, Mississauga, ON). Fractions were then collected and subject to denaturation with Laemmli buffer until further use.

\section{Western Blotting}

For whole-cell lysates in 2x Laemmli buffer, cells were passed six times through 27-gauge needle, heated and proteins resolved through SDS-PAGE with 10\% acrylamide resolving gel. Proteins were then transferred to a PVDF membrane, blocked and incubated with primary and HRP-conjugated secondary antibodies in Tris-buffered saline containing 5\% skimmed milk and 0.1\% Tween-20. Clarity enhanced chemiluminescence (Bio-Rad Laboratories, Mississauga, ON) was used to visualize proteins with ChemiDoc Touch Imaging system (Bio-Rad). Protein quantification was performed using Image Lab software (Bio-Rad) by sequentially normalizing against a loading control and against vehicle-treated condition. Rabbit polyclonal antibodies used were against VAPB (1:3000, HPA013144, Sigma-Aldrich). Mouse monoclonal antibodies used were against clathrin heavy chain (1:500, sc-12734, Santa Cruz Biotechnology, Santa Cruz, CA, USA) and ATP5A (1:2000, ab14748, Abcam). Rat monoclonal antibodies were against LAMP1 (1:200, 1D4B, Developmental Studies Hybridoma Bank, Iowa City, IO). Goat polyclonal antibody used against dynamin 2 (1:1000, sc-6400, Santa Cruz Biotechnology). Secondary antibodies were raised in donkey (Bethyl) and HRP-conjugated. 


\section{Phosphoinositide labelling with ${ }^{3} \mathrm{H}-m y o$-inositol and HPLC-coupled flow scintillation}

RAW cells were incubated for $24 \mathrm{~h}$ with inositol-free DMEM (MP Biomedica, CA) containing $10 \mu \mathrm{Ci} / \mathrm{ml}$ myo-[2- $\left.{ }^{3} \mathrm{H}(\mathrm{N})\right]$ inositol (Perkin Elmer, MA), $1 \mathrm{X}$ insulin-transferrin-seleniumethanolamine (Gibco), 10\% dialyzed FBS (Gibco), 4 mM L-glutamine (Sigma-Aldrich) and 20 mM HEPES (Gibco). Cells were then treated with rotenone, $\mathrm{H}_{2} \mathrm{O}_{2}$ and/or apilimod as indicated. Cells were lysed and lipids precipitated with $600 \mu \mathrm{l}$ of $4.5 \%$ perchloric acid (v/v) for 15 min on ice, collected by scraping and pellet obtained at $12000 \mathrm{x} g$ for $10 \mathrm{~min}$. Then, $1 \mathrm{ml}$ of $0.1 \mathrm{M}$ EDTA was used to wash pellets followed by resuspension in $50 \mu \mathrm{l}$ water. This was followed by $500 \mu \mathrm{l}$ of methanol/40\% methylamine/1-butanol [45.7\% methanol: $10.7 \%$ methylamine: $11.4 \%$ 1butanol (v/v)] used for $50 \mathrm{~min}$ at $53^{\circ} \mathrm{C}$ to deacylate phospholipids. Sample pellets were vaccumdried and washed twice in $300 \mu \mathrm{l}$ water with vaccum-drying. Deacylated phospholipids were extracted from dried sample pellets by resuspending pellet in $450 \mu \mathrm{l}$ water and $300 \mu \mathrm{l} 1$ butanol/ethyl ether/ethyl formate (20:4:1), vortexing 5 min, followed by centrifugation $12000 \mathrm{xg}$ for $2 \mathrm{~min}$ and then the bottom aqueous layer was collected. Extraction was performed three times followed by vaccum-drying the aqueous layer and resuspending lipids in $50 \mu 1$ water. For all treatment samples, equal ${ }^{3} \mathrm{H}$ counts were loaded and separated by HPLC (Agilent Technologies, Mississauga, ON) through 4.6 x 250-mm anion exchange column (Phenomenex, Torrance, CA) using a $1 \mathrm{ml} / \mathrm{min}$ flow rate with a gradient set with water (buffer $\mathrm{A}$ ) and $1 \mathrm{M}\left(\mathrm{NH}_{4}\right)_{2} \mathrm{HPO}_{4}, \mathrm{pH}$ 3.8 (phosphoric acid adjusted) (buffer B) as follows: 0\% B for $5 \mathrm{~min}, 0$ to $2 \% \mathrm{~B}$ for 15 minutes, $2 \% \mathrm{~B}$ for 80 minutes, 2 to $10 \% \mathrm{~B}$ for 20 minutes, $10 \% \mathrm{~B}$ for 30 minutes, 10 to $80 \% \mathrm{~B}$ for 10 minutes, $80 \%$ B for 5 minutes, 80 to $0 \%$ B for 5 minutes. Radiolabel signal was detected with a 1:2 ratio of eluate to scintillant (LabLogic, Brandon, FL) in a $\beta$-RAM 4 (LabLogic) and analyzed 
by Laura 4 software. Each phosphoinositide species detected was normalized against the parent phosphatidylinositol peak as described in (Ho et al., 2016).

\section{Statistical analysis}

All experiments were performed independently at least three times. Respective figure legends indicate number of cells/samples assessed, mean, standard error of mean (s.e.m.) and number of independent experiments. For analysing significant difference between various treatment groups, we used unpaired Student's t-test when comparing two groups only or one-way ANOVA test when comparing multiple treatment conditions in non-normalized controls. Tukey's post hoc test coupled to ANOVA tests was used to evaluate pairwise conditions. Statistical significance was defined as $P<0.05$. Software used for analysis was GraphPad Prism 8 . 
Chapter 3

Results: Lysosome enlargement during PIKfyve inhibition occurs due to coalescence 


\subsection{Introduction}

Pharmacological or genetic depletion of lipid kinase PIKfyve enlarge lysosomes through depletion of the lysosomal membrane lipid PtdIns(3,5) $\mathrm{P}_{2}$ (Ho et al., 2012; Shisheva, 2008). We sought to determine whether such enlargement occurs due to increase in individual lysosome volume while lysosome number remains unchanged, or due to increase in individual lysosome volume while lysosome number decreases (Fig. 9). We employed quantitative 3-dimensional (3D) image analysis using Volocity 6.3.0 software. Specifically, following image acquisition of labelled lysosomes with endocytic tracers, Lucifer yellow and Alexa-conjugated dextran, regions of interest were drawn around cells, and fluorescence intensity threshold and size exclusion threshold were applied to select for labelled lysosomes (Elmquist et al., 1992; Page et al., 1994). This allowed for automated counting of number of labelled lysosomes, and the average voxel count and corresponding average volume of lysosomes. Voxels are 3D individual elements that constitute a 3D image with position, intensity and dimensions associated. Voxels are thus 3D equivalents of $2 \mathrm{D}$ pixel. A finite set of voxels define volume, where volume corresponds to amount of space occupied within an object (Gerber and Peterson, 2008). Thus, lysosome volume, lysosome number per cell, and total lysosome volume per cell could be automated and changes to these parameters in response to various stimulations could be quantitated.

We used quantitative image analysis to observe how lysosome number and volume respond to acute and genetic depletion of $\operatorname{Ptd} \operatorname{Ins}(3,5) \mathrm{P}_{2}$, and how these parameters change in response to PIKfyve reactivation and $\mathrm{PtdIns}(3,5) \mathrm{P}_{2}$ synthesis following acute inhibition. Additionally, we employed image analysis to track lysosomes and observe their dynamics of fusion-fission cycles and "kiss-and-run", and whether "splitting" frequency is impaired during acute PIKfyve inhibition to favor "coalescence" leading to enlargement. Furthermore, we also 
challenged to see whether microtubules and associated motor complexes play a role in regulating such dynamics during PIKfyve inhibition and reactivation as microtubules serve as cytoskeletal tracks for lysosome motility (Mrakovic et al., 2012; Storrie and Desjardins, 1996).

\subsection{Results}

\subsubsection{PtdIns $(3,5) P_{2}$ depletion reduce lysosome number and increase average lysosome volume}

To delineate the changes in lysosome morphology during PIKfyve inhibition, we first exposed RAW macrophages to progressively longer periods of apilimod treatment to inhibit PIKfyve. Using volumetric analysis, we observed a gradual increase in lysosome volume, accompanied by a decrease in lysosome number. Yet, the total lysosome volume per cell remained unchanged (Fig. 10). In addition to acute PtdIns(3,5) $\mathrm{P}_{2}$ depletion, we similarly observed a decrease in lysosome number and an increase in individual lysosome volume in Fig4-

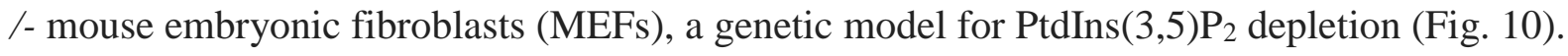
We also observed a similar decrease in lysosome number and increase in individual lysosome volume for HeLa cells treated with apilimod, suggesting that the effect of $\operatorname{PtdIns}(3,5) \mathrm{P}_{2}$ depletion on lysosome number and size was reproducible across a few cell lines, and independent of chronic versus acute PtdIns(3,5) $\mathrm{P}_{2}$ depletion (Fig. 11). This supports our hypothesis that lysosome enlargement during PtdIns(3,5) $\mathrm{P}_{2}$ depletion occurs through lysosome coalescence. 

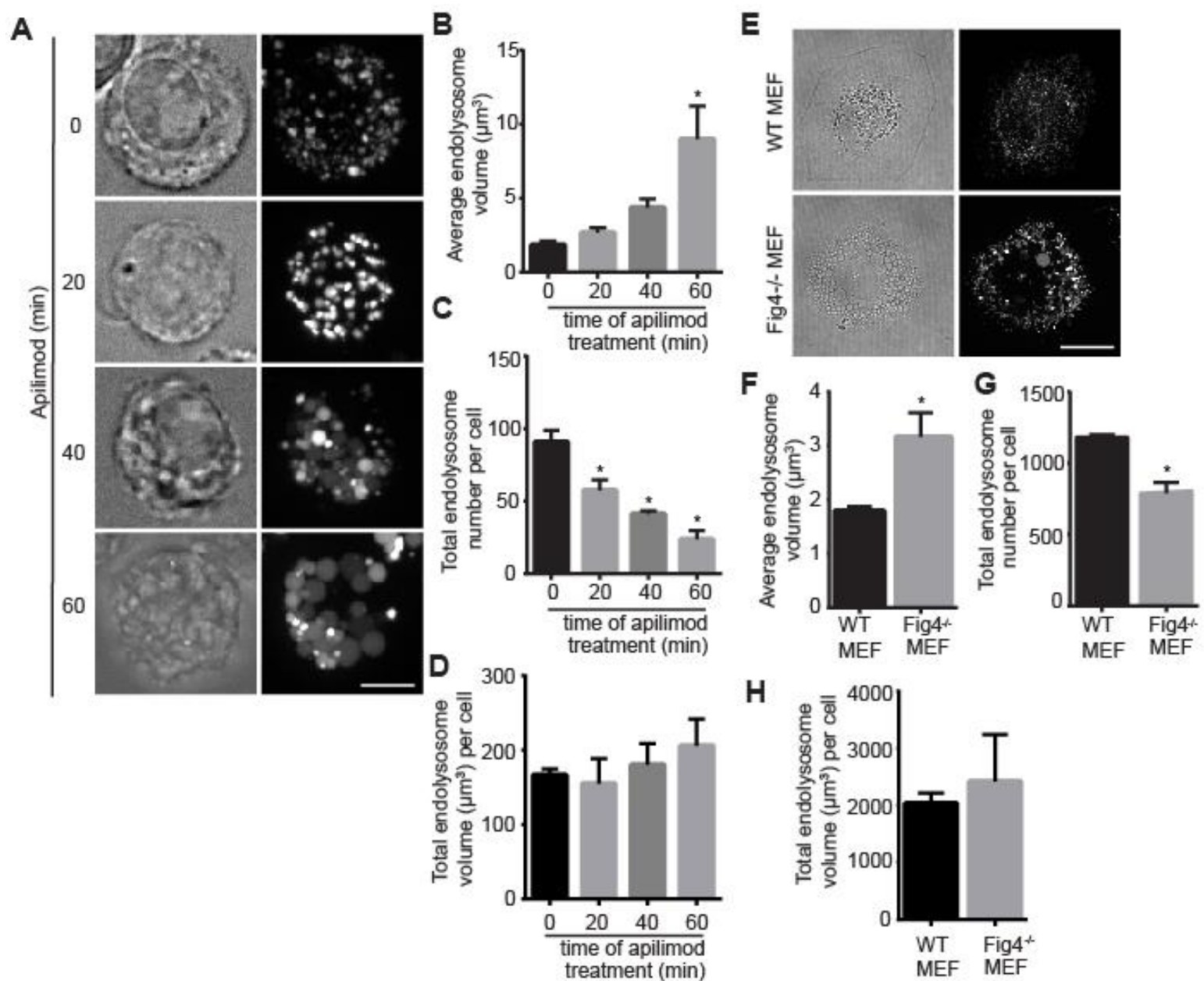

Figure 10: PtdIns(3,5)P2 depletion reduce lysosome number and increase average volume in RAW macrophages. A) Lysosomes of RAW macrophages labelled with Lucifer yellow. The cells were treated with $0.1 \%$ DMSO $1 \mathrm{~h}, 20 \mathrm{nM}$ apilimod for $20 \mathrm{~min}, 40 \mathrm{~min}$ and $60 \mathrm{~min}(\mathrm{n}=3)$. Lysosomes per cell assessed for: B) average volume, C) lysosome number, and D) total lysosome volume (data represent \pm SEM from 3 independent experiments with 15-20 cells counted per condition per experiment, scale bar represents $5 \mu \mathrm{m}$ ). E) Wild-type and Fig4 $4^{-/-}$MEFs labelled with Lucifer yellow for lysosomes. Lysosomes per cell assessed for: F) average volume, G) number, and $\mathbf{H}$ ) total volume (data represent \pm SEM from 3 independent experiments with 15-20 cells counted per condition per experiment, scale bar represents $30 \mu \mathrm{m})$. 
A

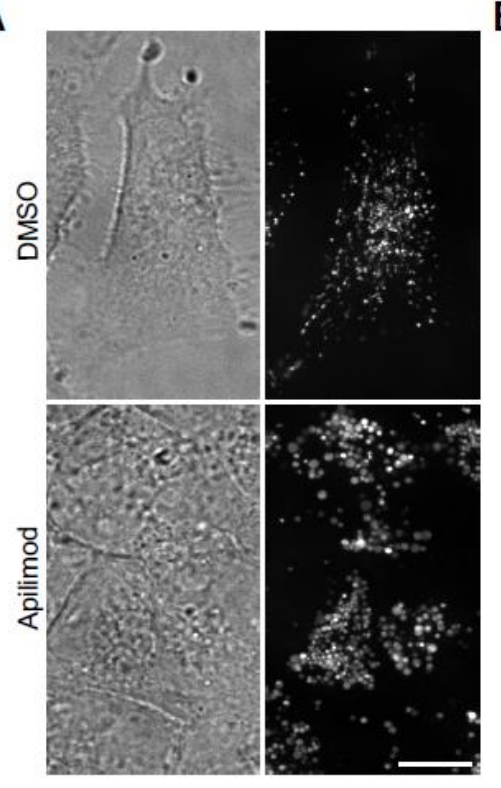

B

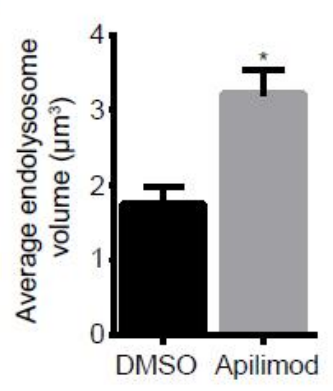

C

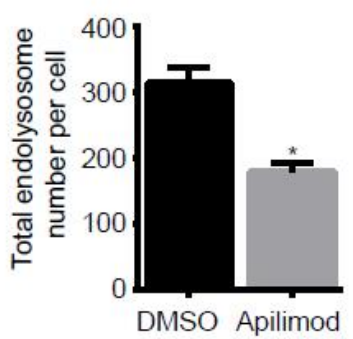

D

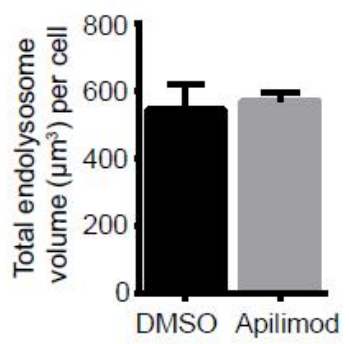

Figure 11: Apilimod treatment reduce lysosome number and increase average volume in

HeLa cells. A) Lysosomes of HeLa cells labelled with Alexa ${ }^{546}$-conjugated dextran. The cells were treated with $0.1 \%$ DMSO or $100 \mathrm{nM}$ apilimod for $60 \mathrm{~min}(\mathrm{n}=3)$. Lysosomes per cell assessed for: B) average volume, C) lysosome number, and D) total lysosome volume (data represent \pm SEM from 3 independent experiments with 15-20 cells counted per condition per experiment, scale bar represents $10 \mu \mathrm{m})$.

\subsubsection{PIKfyve reactivation increase lysosome number and reduce individual lysosome volume}

We next sought to determine if changes in lysosome number and size of individual lysosomes was reversible by removing apilimod to re-activate PIKfyve, which would suggest role of PtdIns $(3,5) \mathrm{P}_{2}$ in lysosomes fragmentation or "fission" . This was done by first treating RAW macrophages that were previously labelled with Lucifer yellow with apilimod for $1 \mathrm{~h}$, followed by 
washing off of the drug with PBS and incubating cells with fresh medium for 0,3 , and $4 \mathrm{~h}$ to reactivate PIKfyve and then imaged as before. We demonstrated that upon $\operatorname{PtdIns}(3,5) \mathrm{P}_{2}$ resynthesis, individual lysosomes decreased in volume and increased in number (Fig. 12); once again, there was no change to total lysosome volume (Fig. 12D). This suggests that lysosomes underwent splitting or fragmentation when $\operatorname{PtdIns(3,5)P2~was~re-synthesized,~supporting~a~role~for~}$ PtdIns(3,5) $\mathrm{P}_{2}$ in lysosome "fission".
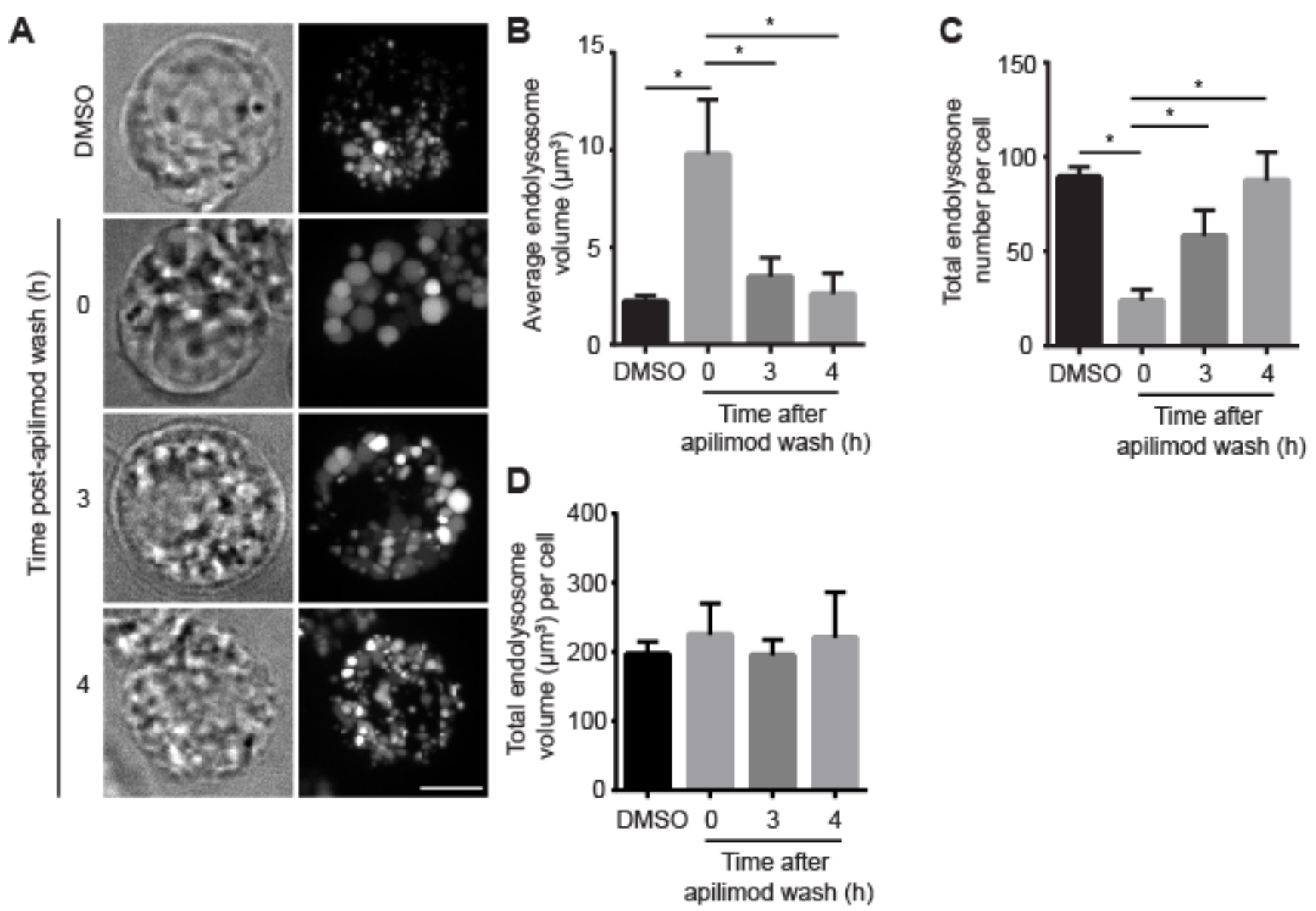

Figure 12: PIKfyve reactivation increase lysosome number and reduce average volume.

A) Lysosomes of RAW macrophages labelled with Lucifer yellow. The cells were treated with 
$0.1 \%$ DMSO $1 \mathrm{~h}$, apilimod $20 \mathrm{nM} 1 \mathrm{~h}, 20 \mathrm{nM}$ apilimod $1 \mathrm{~h}$ followed by removal of apilimod for $3 \mathrm{~h}$ and $4 \mathrm{~h}(\mathrm{n}=3)$. Lysosomes per cell was assessed for: B) average volume and, C) lysosome number, and D) total volume (data represent \pm SEM from 3 independent experiments with 15-20 cells counted per condition per experiment, scale bar represents $5 \mu \mathrm{m}$ ).

\subsubsection{Lysosome undergo constant "kiss-and-run" under regulation by $\operatorname{PtdIns}(3,5) \mathrm{P}_{2}$}

Lysosomes are reported to be highly dynamic and undergo repeated transient "kiss-andrun" for luminal content exchange (Storrie and Desjardins, 1996). Thus, to determine if we could visualize changes in lysosome fission during PIKfyve inhibition and reactivation, we attempted to use conventional spinning disc confocal microscopy to record lysosome dynamics over a continuous time series under normal and apilimod treated conditions. However, we noticed that we could not record lysosome motility over extended and frequent time-lapse imaging, due to photobleaching of fluorophores (fluorophores losing emission signal intensity) and lysosomes not enlarging from apilimod treatment (Fig. 13).

To understand whether these issues result from frequent laser exposure from fluorescence microscopy, we applied mild laser exposure to cells by reducing frequency of imaging and acquiring only single z-focal planes. We observed that such mild laser exposure allowed us to visualize lysosome enlargement due to apilimod treatment (Fig. 14). However, since the image acquisition frequency was very low and long between each time interval, we could not capture how lysosomes behave normally which could be otherwise understood if the intervals between image acquisition frequency were very short. Therefore, we collaborated with Dr. Simon Watkins from University of Pittsburgh; his lab operates a sweptfield confocal fluorescence 
microscope. Sweptfield microscope circumvents the issue of laser photodamage from spinning disc confocal microscope by using a slit that spread the light across the image plane, thus allowing only a single scan using low-light for image acquisition. This allows for imaging at high frequency with short durations between each time interval, allowing visualization of cellular events that occur at high frequency, using low power laser light as the fluorophore excitation source (Castellano-Muñoz et al., 2012). Through sweptfield microscopy, we could observe the normal "kiss-and-run" and/or complete fusion-and-fission behavior of lysosomes under normal and apilimod treated conditions. We applied lysosome tracking and automated lysosome splitting frequency to demonstrate that apilimod reduce lysosome "splitting", suggesting that acute PIKfyve inhibition and PtdIns(3,5) $\mathrm{P}_{2}$ depletion prevents lysosomes from splitting during "kissand-run" dynamics to shift the interactions towards "coalescence" (Fig. 15).

Duration of DMSO treatment (min)

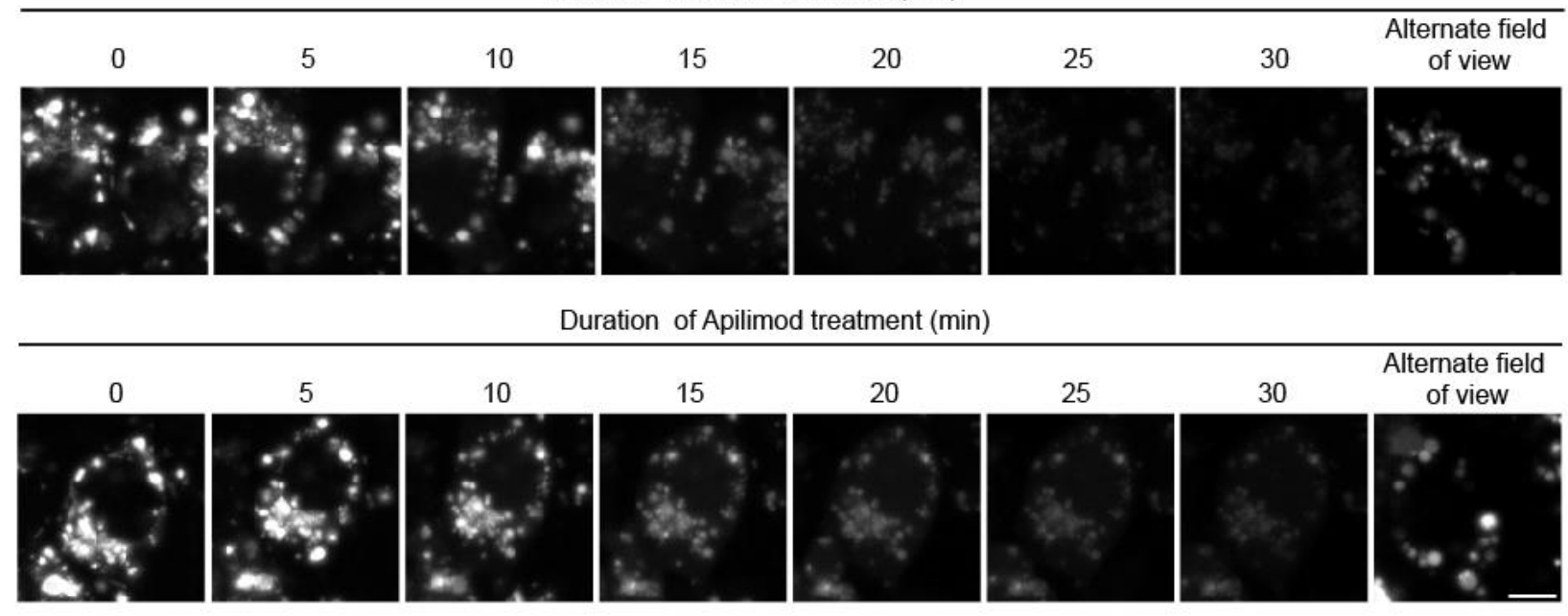

Figure 13: Spinning disc live cell imaging with frequent laser exposure impair lysosome movement. Lysosomes of RAW cells labelled with dextran Alexa ${ }^{546}$-conjugated dextran. Cells were exposed to $0.1 \%$ DMSO or $20 \mathrm{nM}$ apilimod and live cell imaging was performed by 
capturing six z-focal planes with $0.3 \mu \mathrm{m}$ between each plane every $10 \mathrm{sec}$ for $30 \mathrm{~min}(\mathrm{n}=3)$. Select image stills from imaging videos of single cells are shown as well as a single cell from an alternate field of view (scale bar represent $5 \mu \mathrm{m}$ ).

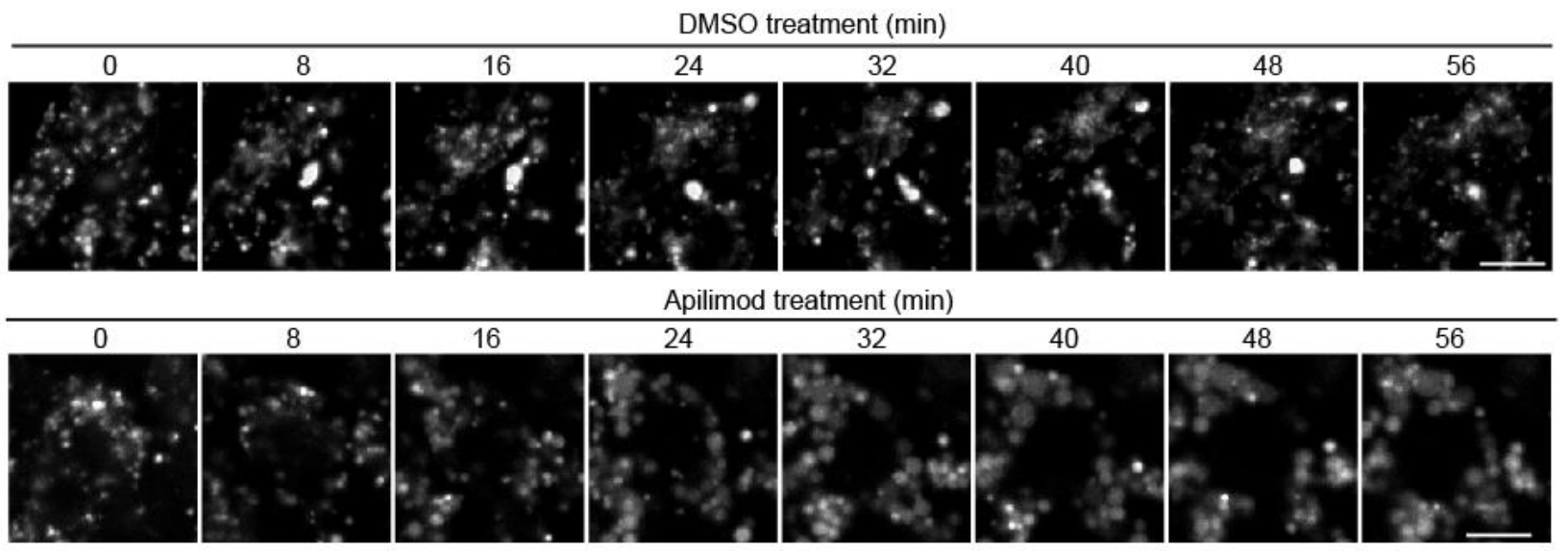

Figure 14: Spinning disc live cell imaging with low frame rate laser exposure to capture apilimod induced lysosome vacuolation. Lysosomes of RAW cells labelled with Alexa ${ }^{546}$ conjugated dextran. Cells exposed to $0.1 \%$ DMSO or 20nM apilimod and live cell imaging performed by capturing single $\mathrm{z}$-focal planes every $2 \min$ for $1 \mathrm{~h}(\mathrm{n}=3)$. Select image stills from imaging videos of single cells are shown. Scale bar: $5 \mu \mathrm{m}$. 

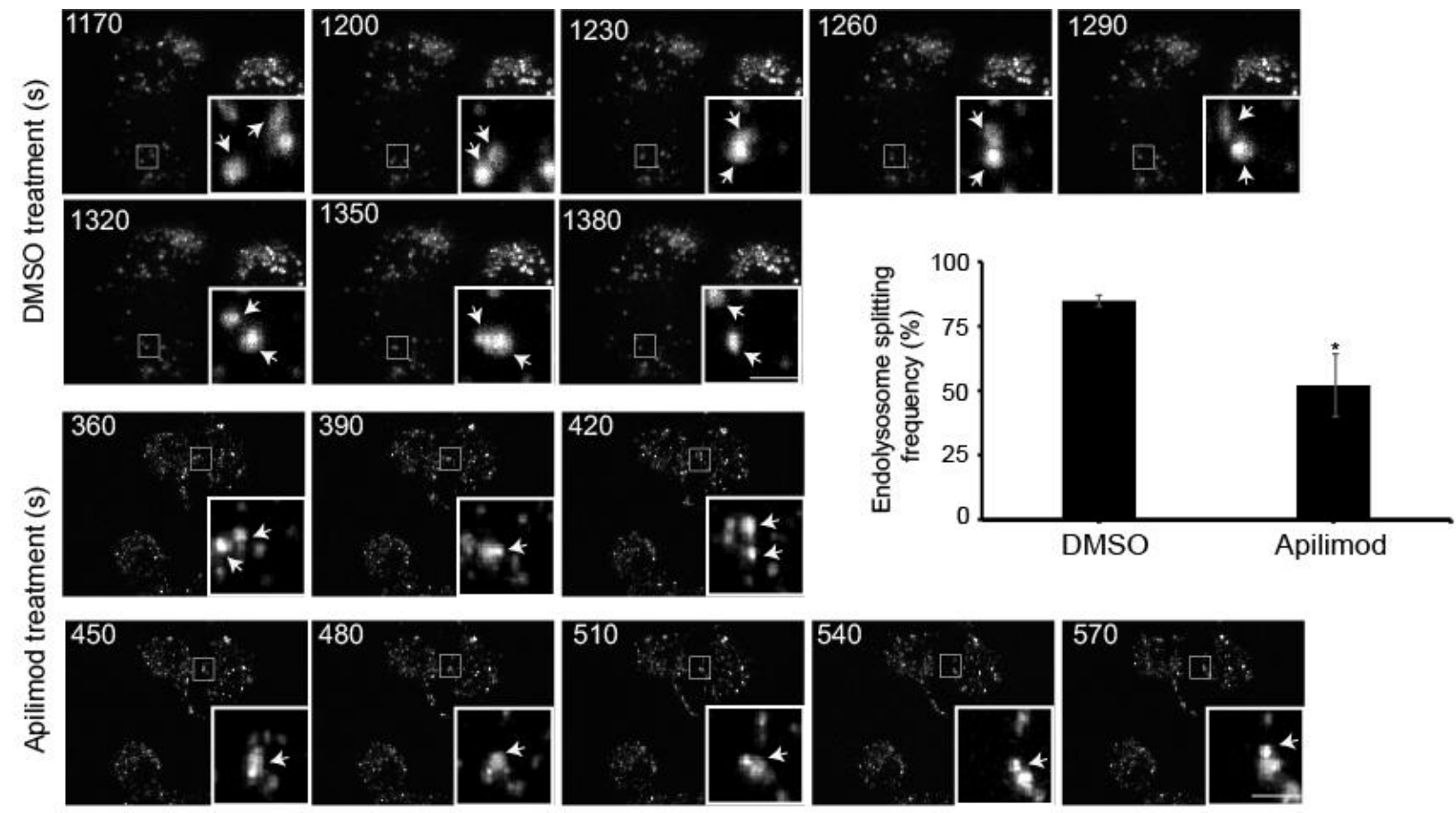

Figure 15: Swept field confocal live imaging to capture apilimod induced lysosome enlargement. Lysosomes of RAW cells labelled with Alexa ${ }^{546}$-conjugated dextran, treated with vehicle (0.1\% DMSO) or 20nM apilimod and imaging was performed across $10 \mathrm{z}$-focal planes every $30 \mathrm{sec}$. Select image stills from imaging videos with zoomed in insets from different timepoints from are shown. Scale bar represent: $3 \mu \mathrm{m}$. Particle tracking analysis to evaluate lysosome splitting/fission across 30 min for each treatment are shown. 


\subsubsection{Lysosome dynamics during PIKfyve inhibition and re-activation is microtubule dependent}

Since lysosomes are reported to undergo microtubule-dependent fusion and fission dynamics, our next objective was to understand whether microtubules are involved in lysosome enlargement during PIKfyve inhibition and lysosome shrinkage during PIKfyve reactivation. Using nocodazole, a microtubule depolymerizing drug, we showed that lysosomes fail to enlarge completely during co-treatment with nocodazole and apilimod (Fig. 16A-C) (Heuser, 1989). Additionally, microtubule depolymerisation during apilimod wash (PIKfyve reactivation) more rapidly fragmented lysosomes and increased their number compared to cells exposed to apilimod-wash condition alone (Fig. 16D-F). These results suggest that lysosomes interact through microtubule tracks, and upon removal of microtubules through nocodazole, lysosomes cannot interact as well to undergo lysosome coalescence during apilimod treatment. However, during PIKfyve reactivation and PtdIns(3,5) $\mathrm{P}_{2}$ synthesis, lysosomes fragment more readily, likely because lysosome fusion events are reduced during microtubule depolymerization, shifting the dynamic toward lysosome splitting even further (Fig. 33). 

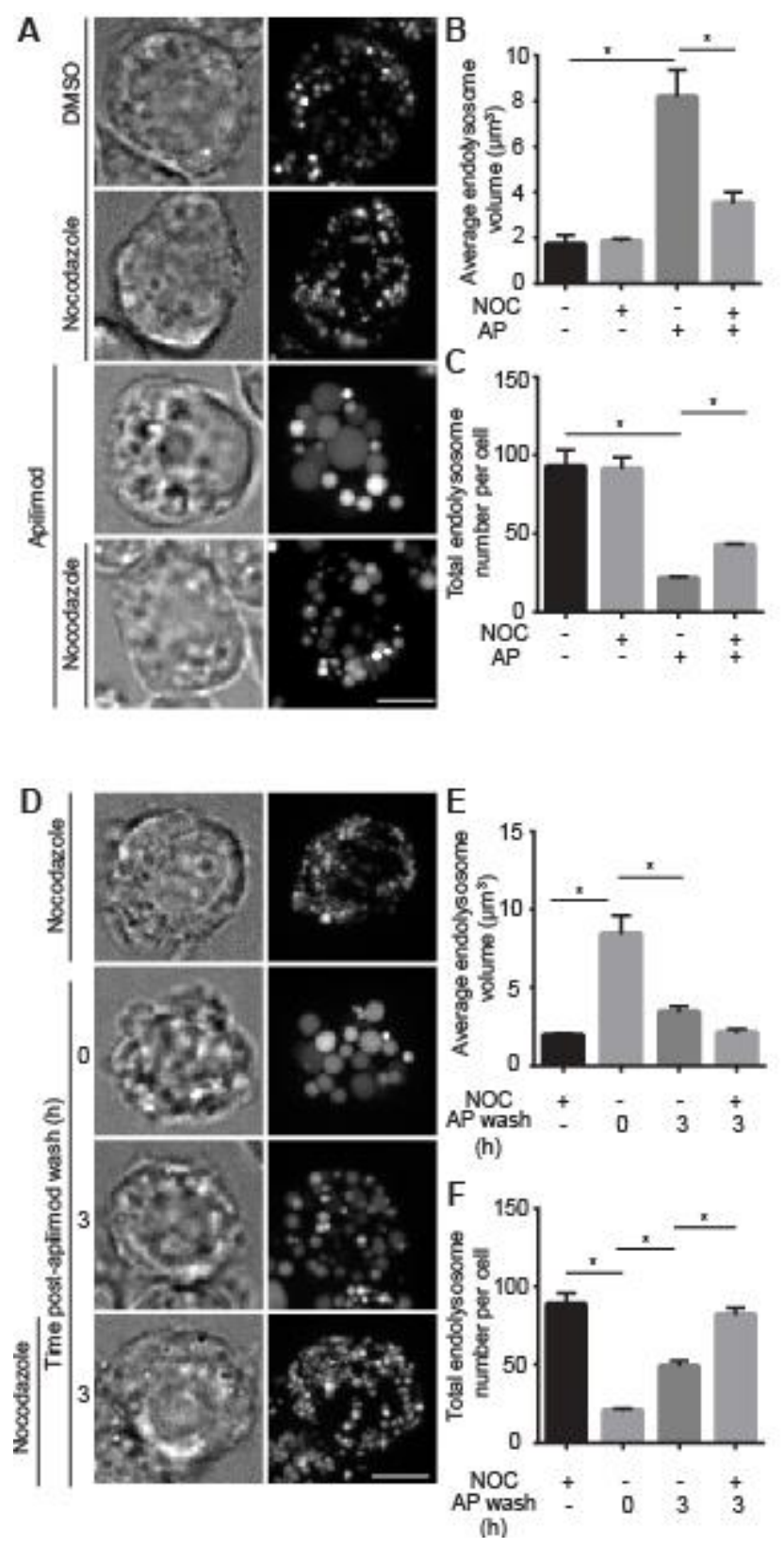

Figure 16: Microtubule regulate lysosome dynamics during PIKfyve inhibition and reactivation. A) Lysosomes of RAW macrophages labelled with Alexa ${ }^{488}$-conjugated dextran. Cells treated with $0.2 \%$ DMSO $1 \mathrm{~h}, 10 \mu \mathrm{M}$ nocodazole $1 \mathrm{~h}, 20 \mathrm{nM}$ apilimod for $1 \mathrm{~h}$ in the presence and absence of $10 \mu \mathrm{M}$ nocodazole ( $\mathrm{n}=3$ ). Lysosomes per cell assessed for: B) average volume and, C) number (data represent \pm SEM from 3 independent experiments with 15-20 cells counted per 
condition per experiment). D) Lysosomes of RAW macrophages labelled with Alexa ${ }^{488}$. conjugated dextran. The cells were treated with $10 \mu \mathrm{M}$ nocodazole for $3 \mathrm{~h}, 20 \mathrm{nM}$ apilimod for 1 $\mathrm{h}$ and imaged immediately after washing away apilimod $(\mathrm{t}=0)$ or after $3 \mathrm{~h}$ in presence or absence of $10 \mu \mathrm{M}$ nocodazole (n=3). Lysosomes per cell assessed for: $\mathbf{E}$ ) average volume and, F) number (data represent \pm SEM from 3 independent experiments with 15-20 cells counted per condition per experiment).

\subsubsection{Microtubule motor complexes regulate lysosome coalescence during PIKfyve inhibition}

Cargo delivery on microtubules occur through motor protein complexes that connect microtubules to the cargo, and use ATP hydrolysis to drive cargo toward the cell periphery through the motor kinesin or towards the perinuclear region through the motor dynein (Pu et al., 2016a). We used the cytoplasmic dynein inhibitor, ciliobrevin $\mathrm{D}$, to demonstrate that dynein is involved in lysosome motility for coalescence to occur during PIKfyve inhibition since lysosome enlargement is abrogated during co-treatment with ciliobrevin D and apilimod (Fig. 17A-C) (Sainath and Gallo, 2015). Additionally, we also transfected RAW macrophages with plasmids expressing Kif5B ${ }^{\mathrm{DN}}$. RFP and p50-dynamitin-GFP, to respectively inhibit kinesin-1 and dynein activity on lysosomes (Echeverri et al., 1996; Mrakovic et al., 2012; Silver and Harrison, 2011). Inhibition of dynein and kinesin-1 through p50-dynamitin-GFP and $\mathrm{Kif5B}^{\mathrm{DN}}$-RFP respectively prevented lysosome coalescence during apilimod treatment (Fig. 17D-I). Thus, in addition to microtubules, associated motor protein complexes function to regulate lysosome motility and help mediate lysosome coalescence during PIKfyve inhibition. 


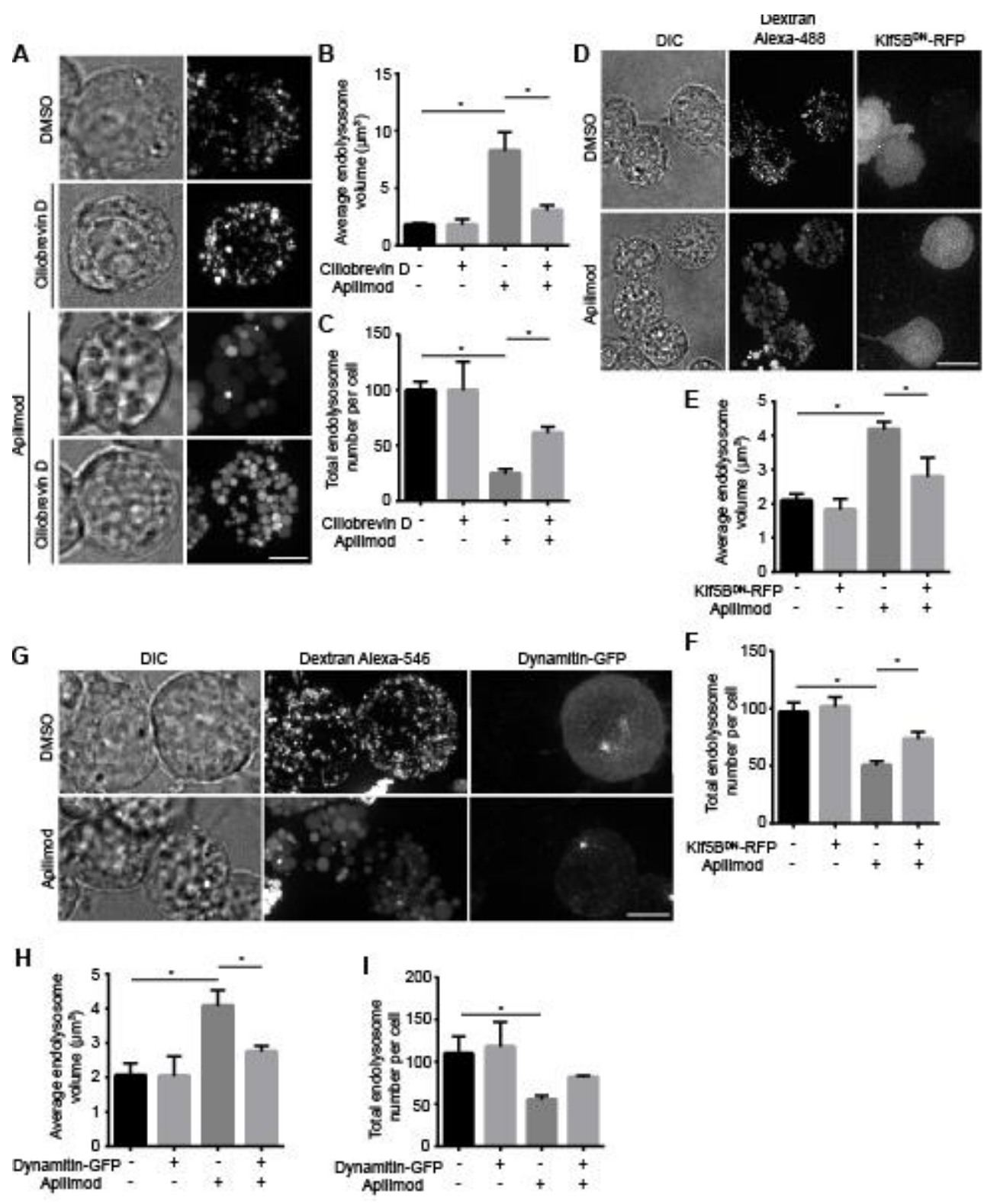

Figure 17: Microtubule motors regulate apilimod induced lysosome enlargement. A) Lysosomes of RAW macrophages labelled with Alexa ${ }^{488}$-conjugated dextran, and treated with: 1) 0.2\% DMSO, 2) Ciliobrevin D $100 \mu \mathrm{M}$ for $2.5 \mathrm{~h}, 3)$ Apilimod $20 \mathrm{nM} 1 \mathrm{~h}$, and 4) Ciliobrevin D $100 \mu \mathrm{M} 2.5 \mathrm{~h}$ with apilimod $20 \mathrm{nM}$ for $1 \mathrm{~h}$. Lysosomes per cell assessed for: B) average volume, and C) number per cell. Scale bar (A): represent $5 \mu \mathrm{m}$. D) RAW cells were transfected with $\mathrm{Kif5}^{\mathrm{DN}}$-RFP and lysosomes labelled with Alexa ${ }^{488}$-conjugated dextran, followed by treatment 
with $0.1 \%$ DMSO or $20 \mathrm{nM}$ apilimod for 30min. Transfected and un-transfected cells were assessed for: E) average lysosome volume and, F) lysosome number per cell. Scale bar (D): 10 $\mu \mathrm{m}$. G) RAW cells transfected with dynamitin-GFP (p50-dynamitin-GFP) and lysosomes labelled with Alexa ${ }^{546}$-conjugated dextran. Cells treated with $0.1 \%$ DMSO or apilimod $20 \mathrm{nM}$ for 30 min. Transfected and un-transfected cells assessed for: $\mathbf{H}$ ) average lysosome volume and, I) lysosome number per cell. Scale bar (G): $5 \mu \mathrm{m}$. (For all experiments, data represent \pm SEM from 3 independent experiments with 15-20 cells counted per condition per experiment). 


\section{Chapter 4: Reactive oxygen species regulate lysosome fusion-fission}




\subsection{Introduction}

We demonstrated that PtdIns(3,5) $\mathrm{P}_{2}$ depletion enlarges lysosomes due to expansion of individual lysosome volume with concurrent decrease in lysosome number while total lysosome volume remains constant. Upon attempting to capture the dynamics of lysosome enlargement through acute PIKfyve inhibition through apilimod using conventional spinning disc fluorescence confocal microscopy, we observed arrested enlargement in field of view exposed to frequent laser light while enlargement proceeded in alternate field of view unexposed to laser (Fig. 13). Photodamage from frequent excitation of fluorophores through florescence microscopy is reported to produce ROS (Icha et al., 2017). ROS can locally signal to lysosome calcium ion channel TRPML1 for lysosome biogenesis, impair lysosome-autophagosome fusion, and can structurally alter actin and microtubules, cytoskeletal tracks for lysosome fusion and fission regulation (Hamacher-Brady et al., 2011; Pocasap et al., 2018; Wilson and González-Billault, 2015; Zheng et al., 2016). We stimulated cells with $\mathrm{H}_{2} \mathrm{O}_{2}$, rotenone to inhibit mitochondrial respiratory electron transport chain (ETC) complex I, 1-chloro-2,4,-dinitrobenzene (CDNB) to inhibit thioredoxin reductase, and monochlorobimane (MCB) to inhibit glutathione (GSH) activity. Rotenone, CDNB, and $\mathrm{MCB}$ produce superoxide, $\mathrm{H}_{2} \mathrm{O}_{2}$, and hydroxyl radical, whereas $\mathrm{H}_{2} \mathrm{O}_{2}$ produce hydroxyl radical and not superoxide (Fig. 8) (Chatterjee et al., 1999; Heinz et al., 2017; Kamencic et al., 2000; Padgaonkar et al., 2015). Therefore, we monitored and tested the hypothesis whether different sources of ROS stimulation impair lysosome "coalescence" and/or accelerate lysosome “fragmentation" during acute PIKfyve inhibition. 


\subsection{Results}

\subsubsection{Frequent laser excitation from fluorescence microscopy produce ROS}

To explain our observation that frequent laser excitation from fluorescence microscopy arrest apilimod induced lysosome enlargement and correlate ROS production as the causative agent for arrest of enlargement, we used the nitroblue tetrazolium (NBT) to monitor ROS production from fluorescence microscopy. NBT is reduced by superoxide to form formazan crystal that can be visualized through far-red excitation/emission filter (Sim Choi et al., 2006). NBT fluorescence intensity increased in response to increased frequency of laser excitation for dextran labelled lysosomes from spinning disc fluorescence imaging, suggestive of ROS production from frequent laser excitation of fluorophores.

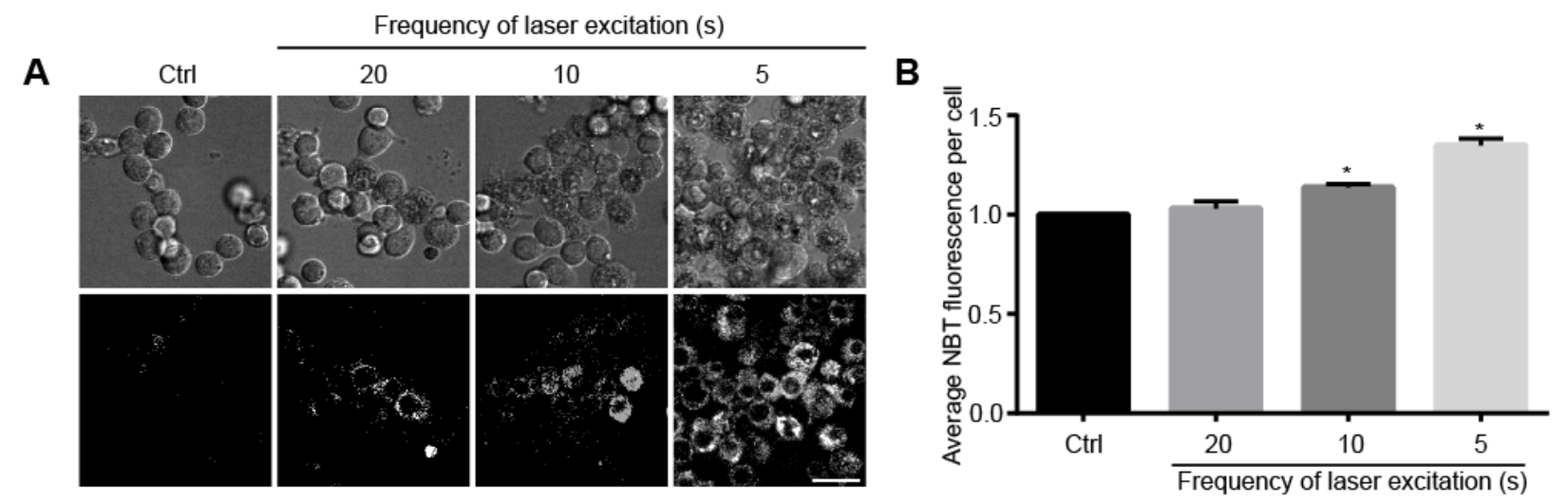

Figure 18: Microscopy induced photo-damage causes ROS production. A) RAW cells incubated with Alexa ${ }^{546}$-conjugated dextran, treated with $1 \mathrm{mg} / \mathrm{ml}$ NBT for $30 \mathrm{~min}$ in the dark, followed by laser excitation of Alexa ${ }^{546}$-conjugated dextran every $20 \mathrm{~s}$ or $10 \mathrm{~s}$ or $5 \mathrm{~s}$ or not excited (ctrl), and NBT fluorescence was detected using far-red confocal channel. Scale bar: $20 \mu \mathrm{m}$. B) 
The cells assessed for average NBT fluorescence per cell (data represent \pm SEM from 3 independent experiments with 20-35 cells counted per condition per experiment).

\subsubsection{ROS stimulation arrest lysosome enlargement from acute PIKfyve inhibition}

We stimulated ROS through four different applications with distinct targets for ROS production: $\mathrm{H}_{2} \mathrm{O}_{2}$, rotenone, $\mathrm{CDNB}$, and $\mathrm{MCB}$, and tested whether these agents could arrest lysosome enlargement from acute PIKfyve inhibition through apilimod. Using the redox-sensitive CellROX Green dye, we observed increase ROS burst from $\mathrm{H}_{2} \mathrm{O}_{2}$, rotenone, CDNB and MCB (Fig. 19A-B). Additionally, MCB through conjugation with GSH released detectable fluorescence emission (Fig. 19C). Strikingly, all four ROS agonists prevented apilimod induced lysosome enlargement for RAW cells. Specifically, apilimod induced lysosome enlargement through growth of individual lysosome volume with concurrent decrease in lysosome number. However, total lysosome volume per cell was unaffected. Co-application of any four ROS agonists and apilimod caused lysosomes to be smaller and numerous compared to apilimod treatment alone (Fig. 20). To examine whether ROS was the causative agent to inhibit apilimod induced lysosome enlargement, we used the ROS scavenger $N$-acetyl- $L$-cysteine (NAC) to demonstrate that the inhibitory effect of at least one of the ROS agonists, rotenone on apilimod induced lysosome enlargement, reversed upon NAC co-administration (Sun, 2010). Specifically, cells treated with NAC, rotenone, and apilimod displayed larger lysosomes than rotenone and apilimod (Fig. 21). To demonstrate that these observations are not restricted to murine macrophages, we show that in addition to RAW cells, ROS stimulation through $\mathrm{H}_{2} \mathrm{O}_{2}$ and CDNB resist lysosome enlargement from apilimod in HeLa cells (Fig. 22A-D) and RPE cells (Fig. 22E-H). Total lysosome volume per cell was unaffected across all experiments involved assessing lysosome volume and number (Fig. 20-22). 
Therefore, ROS stimulation through agonists with distinct targets arrest apilimod induced lysosome coalescence.

A
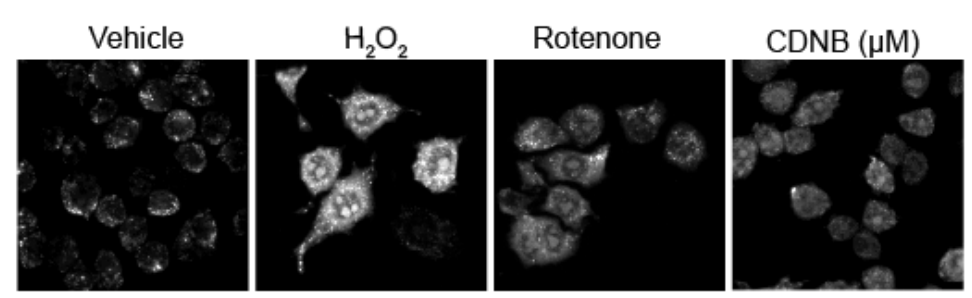

B
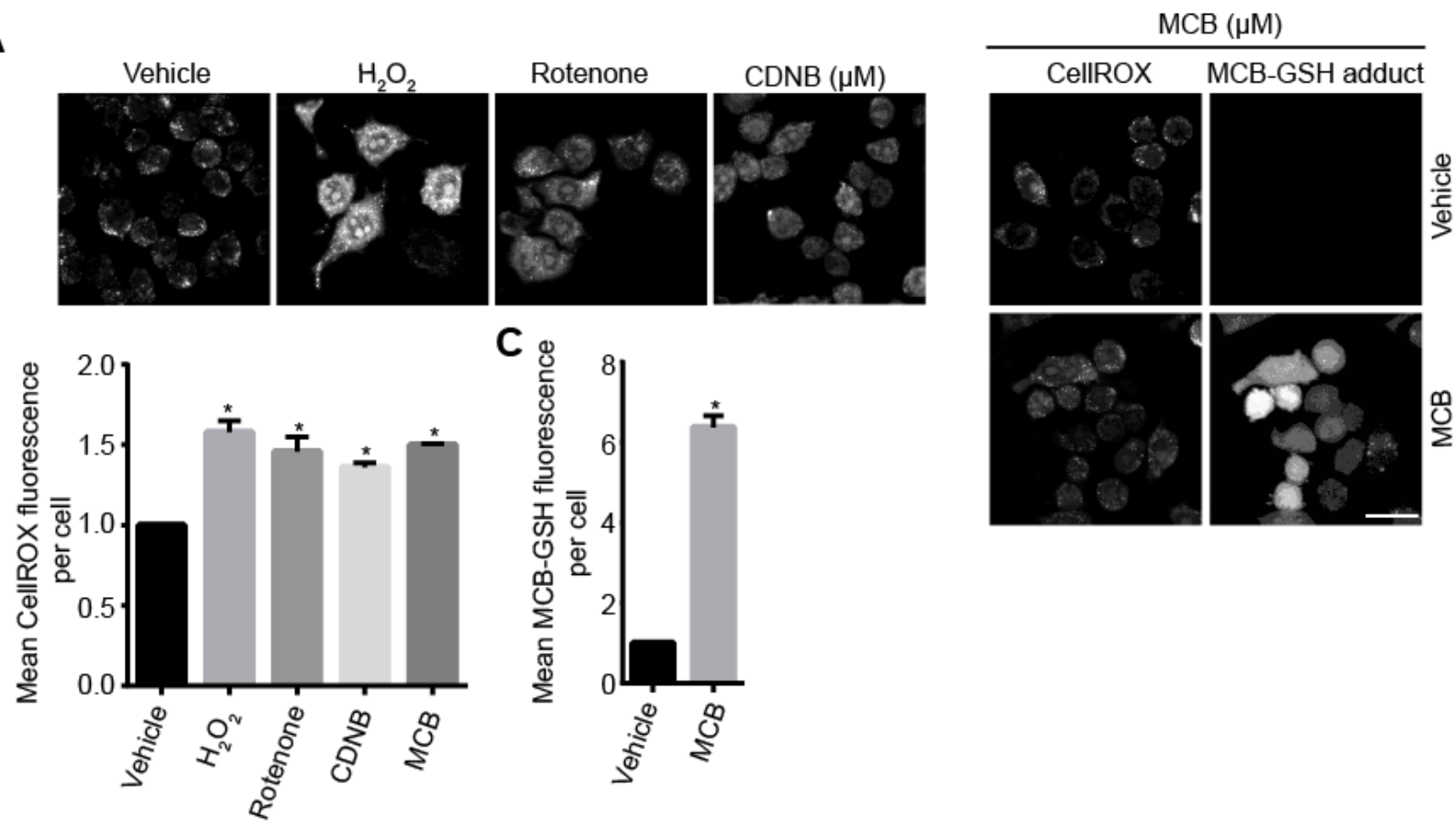

Figure 19: ROS stimulation through pharmacological manipulation. A) RAW cells were treated with vehicle, $1 \mathrm{mM} \mathrm{H} \mathrm{O}_{2} 40 \mathrm{~min}, 10 \mu \mathrm{M}$ CDNB $30 \mathrm{~min}$, or $5 \mu \mathrm{M}$ MCB $30 \mathrm{~min}$ in the presence of $5 \mu \mathrm{M}$ CellROX green for $30 \mathrm{~min}$. Scale bar represent $20 \mu \mathrm{m}$. B) Average CellROX green fluorescence intensity per cell from (A) was quantified and normalized to vehicle treated cells. C) Average MCB-GSH fluorescence emission from (A) quantified and normalized to vehicle treated cells. For both B and C, Data represent \pm SEM from 3 independent experiments with 4050 cells counted per condition per experiment. 


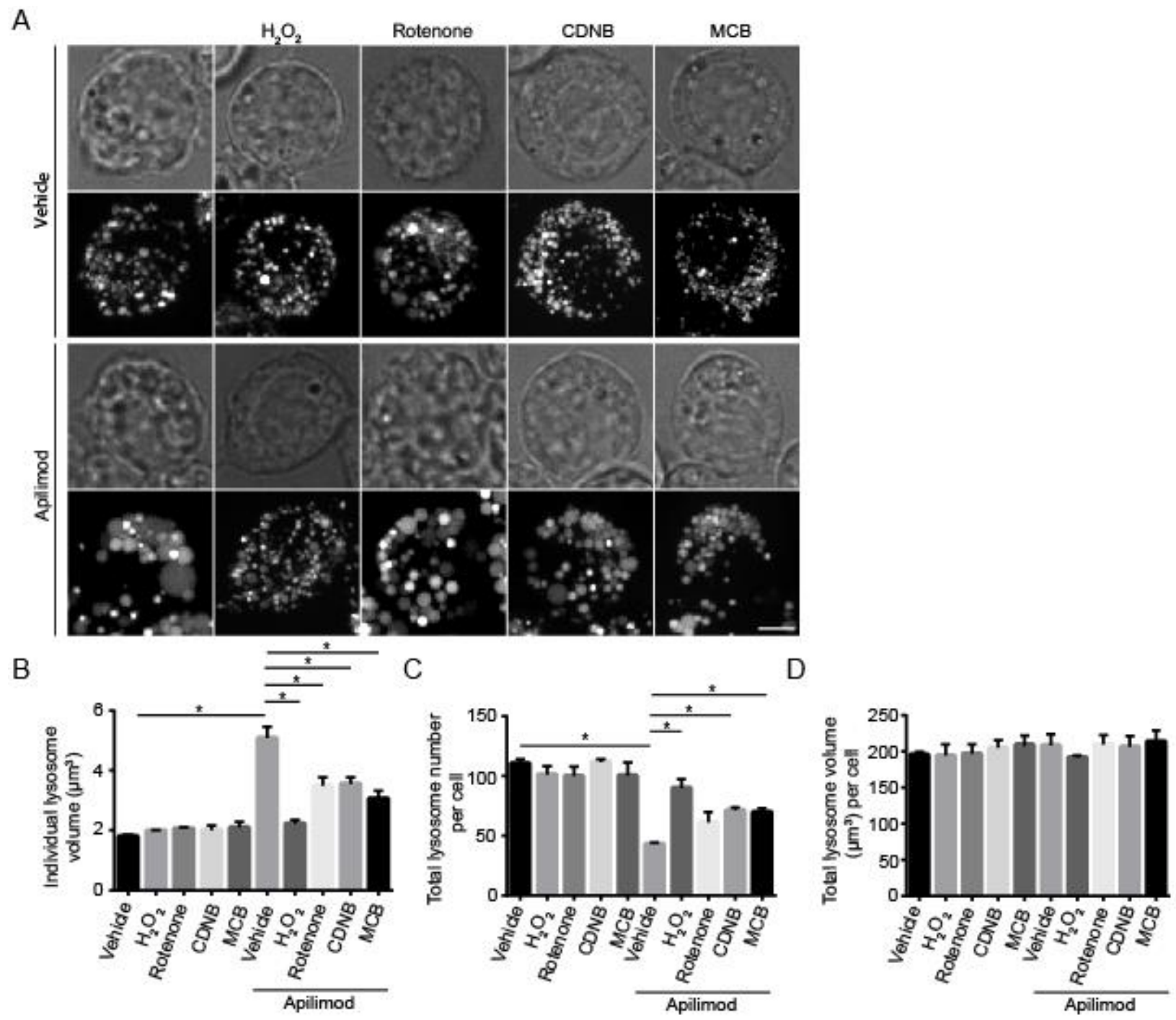

Figure 20: ROS agonists prevent lysosome enlargement during acute PIKfyve suppression.

A) RAW cells pre-labelled with Lucifer yellow and exposed to vehicle or $20 \mathrm{nM}$ apilimod for 40 min. These conditions were then supplemented with additional vehicle or $1 \mathrm{mM} \mathrm{H}_{2} \mathrm{O}_{2}$ for 40 min, $1 \mu \mathrm{M}$ rotenone for $60 \mathrm{~min}, 10 \mu \mathrm{M} \mathrm{CDNB}$ for $30 \mathrm{~min}$, or $5 \mu \mathrm{M} \mathrm{MCB}$ for $30 \mathrm{~min}$. Fluorescence micrographs are represented as z-projections of 45-55 z-plane images obtained by spinning disc microscopy. Scale bar: $5 \mu \mathrm{m}$. B-D: Quantification of individual lysosome volume (B), lysosome number per cell $(\mathbf{C})$, and total lysosome volume per cell (D). Data represent mean \pm S.E.M. from three independent experiments, with 25-30 cells assessed per treatment condition per experiment. 
A
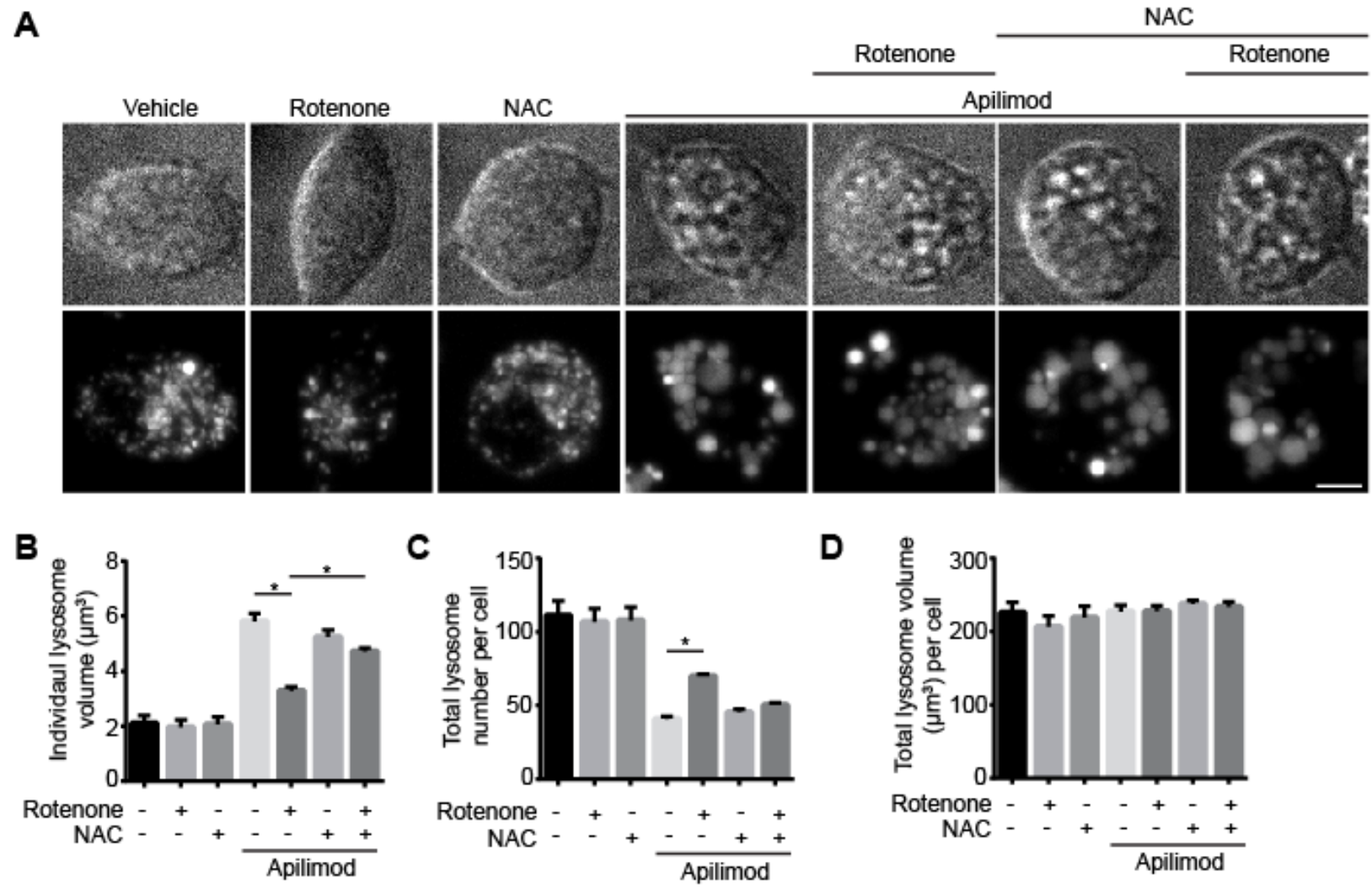

Figure 21. ROS scavengers permit lysosome coalescence during acute PIKfyve suppression.

A) RAW cells pre-labelled with Lucifer yellow and exposed to vehicle, or $0.5 \mu \mathrm{M}$ rotenone 60 min, or $10 \mathrm{mM} \mathrm{N}$-acetyl-L-cysteine (NAC) $120 \mathrm{~min}$ alone, or in presence of $20 \mathrm{nM}$ apilimod for the last $40 \mathrm{~min}$. Fluorescence micrographs are represented as z-projections of $45-55 \mathrm{z}$-plane images obtained by spinning disc microscopy. Scale bar: $5 \mu \mathrm{m}$. B-D: Quantification of individual lysosome volume (B), lysosome number per cell (C), and total lysosome volume per cell (D). Data represent mean \pm S.E.M. from three independent experiments, with $25-30$ cells assessed per treatment condition per experiment. 

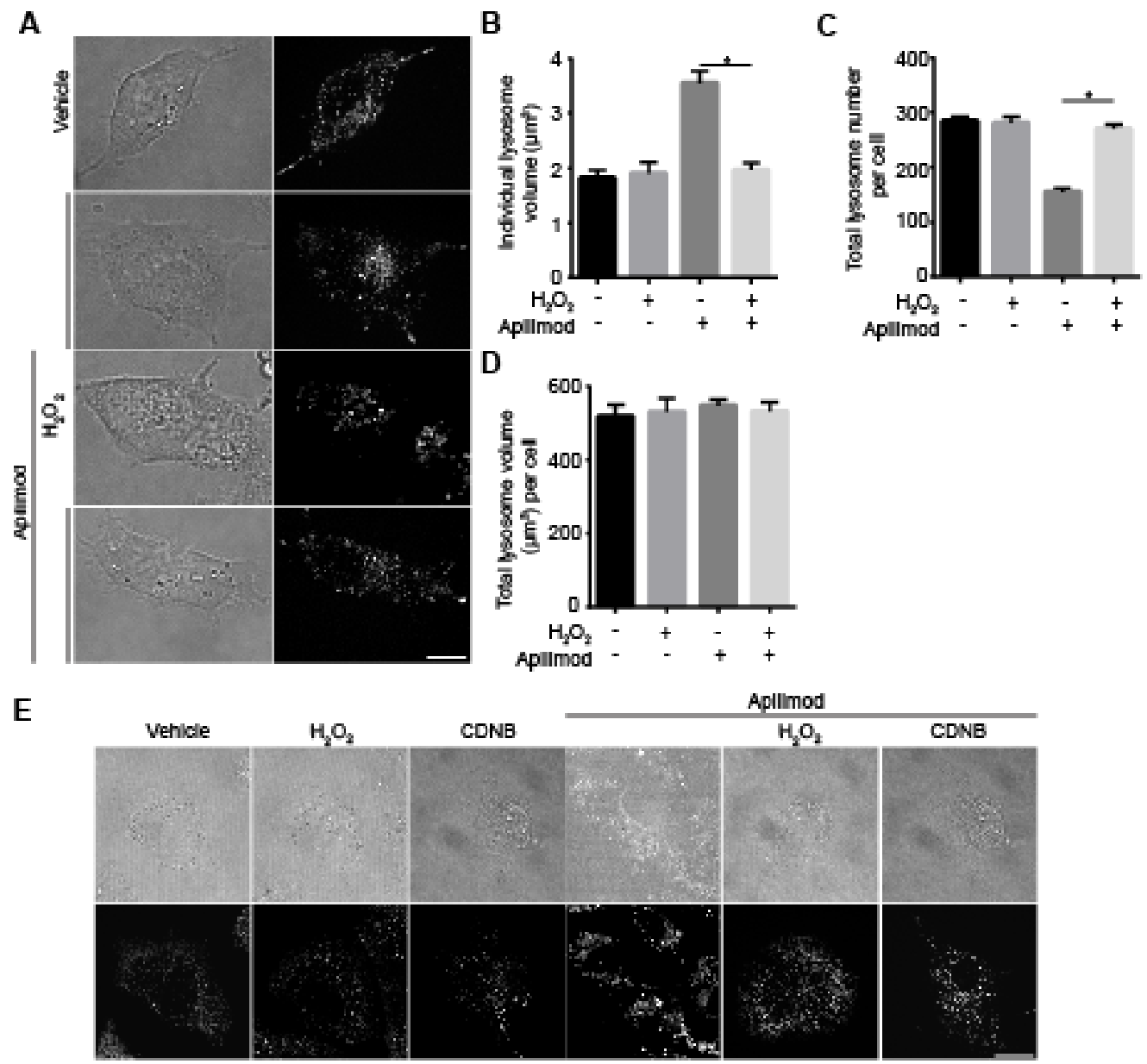

Apllimod
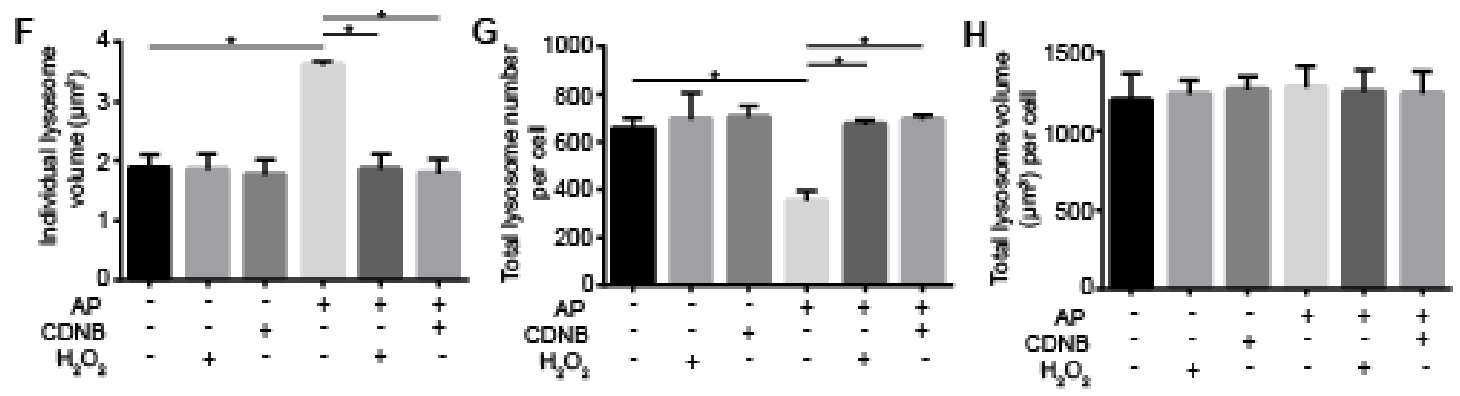

Figure 22: ROS prevent lysosome enlargement during acute PIKfyve suppression in HeLa

and RPE cells. (A) HeLa cells pre-labelled with Lucifer yellow and exposed to vehicle or 100 $\mathrm{nM}$ apilimod $40 \mathrm{~min}$, or with $1 \mathrm{mM} \mathrm{H}_{2} \mathrm{O}_{2}$ in the presence or absence of $100 \mathrm{nM}$ apilimod for 40 min. Scale bar: $10 \mu \mathrm{m}$. (B-D) Quantification of individual lysosome volume per lysosome (B), lysosome number per cell (C), and total lysosome volume per cell (D). (E) RPE cells prelabelled with Lucifer yellow and exposed to vehicle, or $1 \mathrm{mM} \mathrm{H}_{2} \mathrm{O}_{2}$, or $10 \mu \mathrm{M} \mathrm{CDNB}$, in 
presence or absence of $200 \mathrm{nM}$ apilimod $40 \mathrm{~min}$. Scale bar: $20 \mu \mathrm{m}$. (F-H) Quantification of individual lysosome volume (F), lysosome number per cell (G), and sum lysosome volume per cell (H). For (E-H) and (A-D), data are represented as mean s.e.m. from three independent experiments, with 15-20 cells assessed for (F-H) and 25-30 cells assessed for (B-D) per treatment condition per experiment.

\subsubsection{ROS stimulation arrest lysosome enlargement from acute PIKfyve inhibition without neutralizing apilimod or enhancing PtdIns(3,5)P2 synthesis}

We evaluated the possibility that ROS stimulation within cells may impair the structural functionality of apilimod. To test this possibility, we performed in vitro reaction by incubating apilimod and $\mathrm{H}_{2} \mathrm{O}_{2}$ in complete media for 40 min followed by catalase addition to degrade $\mathrm{H}_{2} \mathrm{O}_{2}$. This reaction mixture added to RAW cells and observed whether the functional integrity apilimod preserved to induce lysosome enlargement. Indeed, apilimod was able to induce lysosome enlargement in terms of expansion of individual lysosome volume and reduction in lysosome number, comparable to apilimod alone treatment (Fig. 23A-D), suggesting that $\mathrm{H}_{2} \mathrm{O}_{2}$ did not impair apilimod functional integrity. In addition, co-incubation of $\mathrm{H}_{2} \mathrm{O}_{2}$ with apilimod suppressed apilimod induced lysosome enlargement, while applying in vitro reaction mixture of $\mathrm{H}_{2} \mathrm{O}_{2}$ with catalase followed by addition of apilimod to RAW cells caused lysosome enlargement, further suggesting $\mathrm{H}_{2} \mathrm{O}_{2}$ do not degrade the functional integrity of apilimod (Fig. 23A-D). Therefore, our observations suggest ROS arrest apilimod induced lysosome enlargement without affecting the functional capability of apilimod. 
As discussed, ROS may perturb protein and lipid phosphatase structure through oxidation of cysteine residues (Schieber and Chandel, 2014). We set to observe the possibility of ROS inactivating Fig4 phosphatase, thereby enhancing PtdIns(3,5) $\mathrm{P}_{2}$ synthesis to counteract lysosome coalescence (Sbrissa et al., 2007). We applied rotenone or $\mathrm{H}_{2} \mathrm{O}_{2}$ to RAW cells in absence or presence apilimod and measured PtdInsP synthesis through ${ }^{3} \mathrm{H}-m y o-$ inositol incorporation and HPLC-coupled flow scintillation (Ho et al., 2016). We observed no detectable increase in PtdIns(3,5) $\mathrm{P}_{2}$ synthesis for either rotenone or $\mathrm{H}_{2} \mathrm{O}_{2}$ (Fig. 23E-F), suggesting ROS stimulation do not increase PtdIns $(3,5) \mathrm{P}_{2}$ synthesis to counteract apilimod induced lysosome coalescence. Apilimod cause a spike in PtdIns(3)P synthesis with simultaneous decrease in PtdIns(3,5) $\mathrm{P}_{2}$ levels due to impaired PtdIns(3)P turnover to synthesize PtdIns(3,5) $\mathrm{P}_{2}$. However, $\mathrm{H}_{2} \mathrm{O}_{2}$ prevented such increase in PtdIns(3)P from apilimod. This effect was not significant and not reproducible from rotenone (Fig. 23E-F). This observation is not clear to us and not likely to explain inhibition of lysosome enlargement from apilimod. Therefore, ROS stimulation prevent apilimod induced lysosome coalescence through a mechanism that does not rely on enhanced PtdIns(3,5) $\mathrm{P}_{2}$ synthesis. Previously, our work demonstrated lysosome coalescence was reversible through removal of apilimod and PIKfyve re-activation. We therefore attempted to observe next whether ROS accelerate lysosome fragmentation upon apilimod removal. 
A

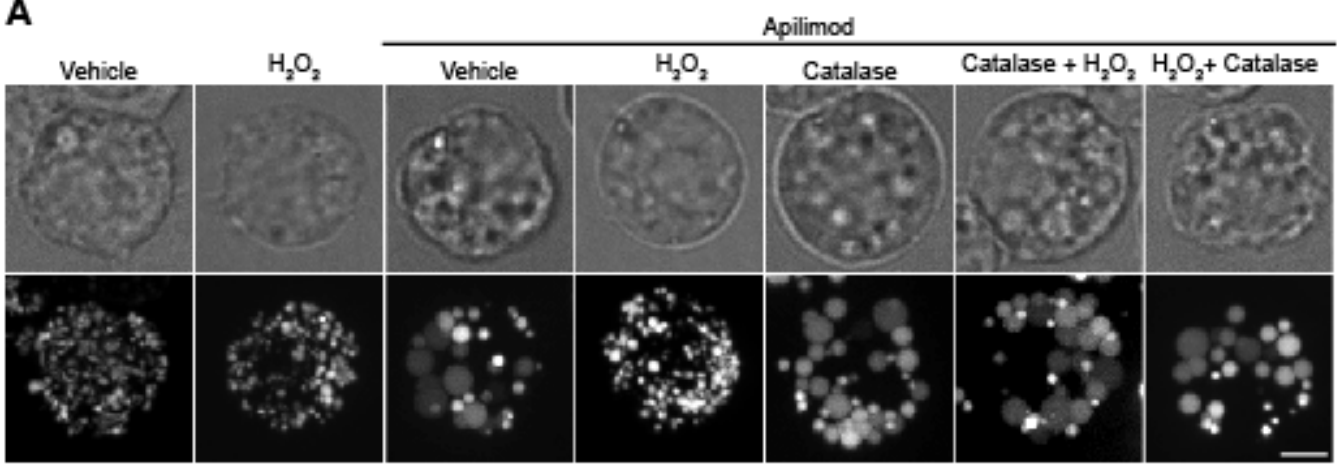

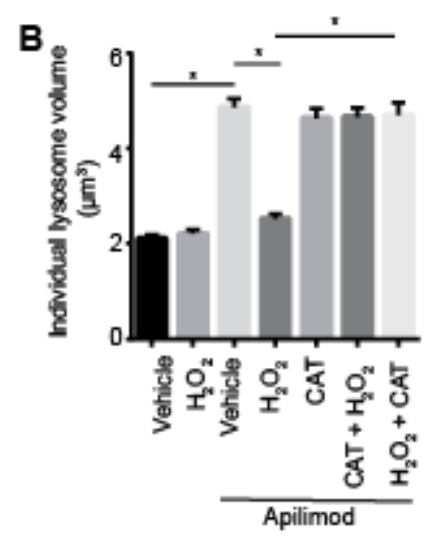

E

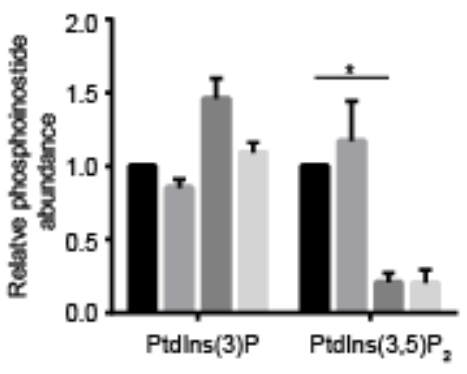

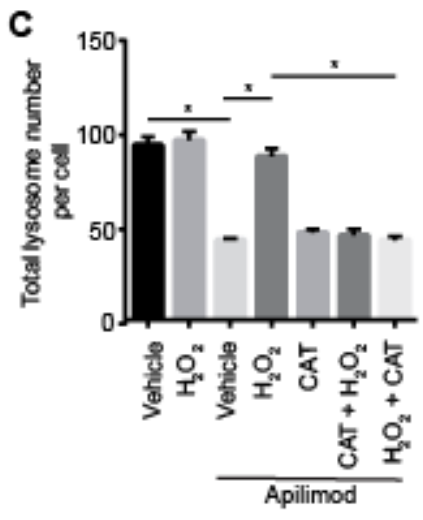

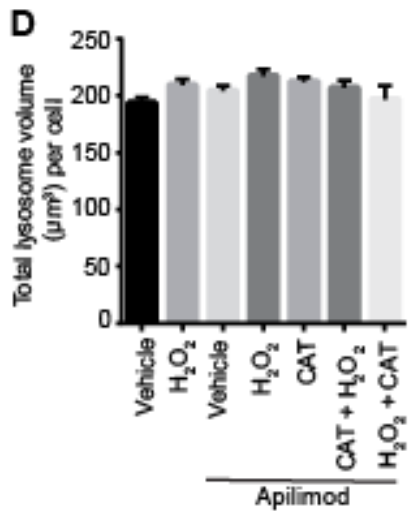

F

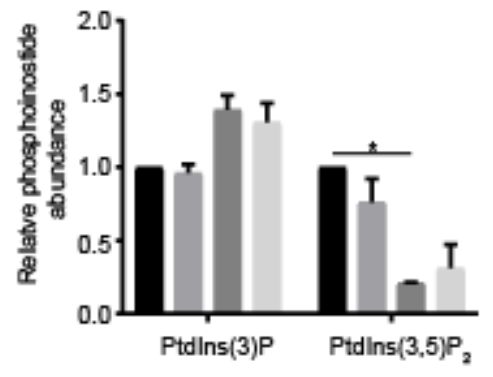

Vehicle

Rotenone

- Api mod

Apilimod +

Rotenone

Figure 23: Apilimod integrity and PtdIns(3,5)P2 levels are not altered by ROS. (A) RAW

cells pre-labelled with Lucifer yellow. Following reactions were performed in complete media in vitro for designated time, prior to adding to cells for an additional 40 min: vehicle; $1 \mathrm{mM} \mathrm{H}_{2} \mathrm{O}_{2}$ $40 \mathrm{~min} ; 20 \mathrm{nM}$ apilimod $40 \mathrm{~min} ; 20 \mathrm{nM}$ apilimod preincubated with $1 \mathrm{mM} \mathrm{H} \mathrm{O}_{2}$ for $40 \mathrm{~min} ; 20$ $\mathrm{nM}$ apilimod preincubated with $0.5 \mathrm{mg} / \mathrm{L}$ catalase for $60 \mathrm{~min} ; 1 \mathrm{mM} \mathrm{H} \mathrm{O}_{2}$ exposed to $0.5 \mathrm{mg} / \mathrm{L}$ catalase for $60 \mathrm{~min}$ to neutralize $\mathrm{H}_{2} \mathrm{O}_{2}$, followed by $20 \mathrm{nM}$ apilimod $40 \mathrm{~min}$; or $20 \mathrm{nM}$ apilimod exposed to $1 \mathrm{mM} \mathrm{H} \mathrm{O}_{2}$ for 40 min to test whether $\mathrm{H}_{2} \mathrm{O}_{2}$ degraded apilimod, followed by 0.5 
$\mathrm{mg} / \mathrm{L}$ catalase for 60 min to degrade $\mathrm{H}_{2} \mathrm{O}_{2}$. Fluorescence micrographs are spinning disc microscopy images with 45-55 z-planes represented as z-projections. Scale bar: $5 \mu \mathrm{m}$. (B-D) Quantification of individual lysosome volume (B), lysosome number per cell (C), and total lysosome volume per cell (D). AP (apilimod), CAT (catalase). Data are shown as mean \pm s.e.m. from three independent experiments, with 25-30 cell assessed per treatment condition per experiment. (E-F) ${ }^{3} \mathrm{H}-m y o$-inositol incorporation followed by HPLC-coupled flow scintillation used to determine PtdIns(3)P and PtdIns(3,5) $\mathrm{P}_{2}$ levels from RAW cells exposed to vehicle alone, or $1 \mathrm{mM} \mathrm{H}_{2} \mathrm{O}_{2} 40 \min (\mathbf{E})$, or $1 \mu \mathrm{M}$ rotenone $60 \min (\mathbf{F})$, in presence or absence of $20 \mathrm{nM}$ apilimod. Data represent \pm s.d. from three independent experiments.

\subsubsection{ROS increase lysosome fragmentation upon apilimod removal}

Lysosome coalescence from apilimod increase individual lysosome volume with concurrent decrease in lysosome number. Conversely, removal of apilimod decrease volume of individual lysosome with increase in lysosome number. To determine whether ROS can increase lysosome fragmentation upon removal of apilimod, following apilimod treatment, we washed away apilimod and added ROS agonists during terminal duration of apilimod wash. Strikingly, all four ROS agonists enhanced lysosome fragmentation upon apilimod wash by accelerating decrease in volume of individual lysosomes with simultaneous increase in lysosome number (Fig. 24). Thus, ROS stimulation can impair lysosome coalescence and/or increase lysosome fragmentation by hindering fusion and/or increasing fission rates. 
A

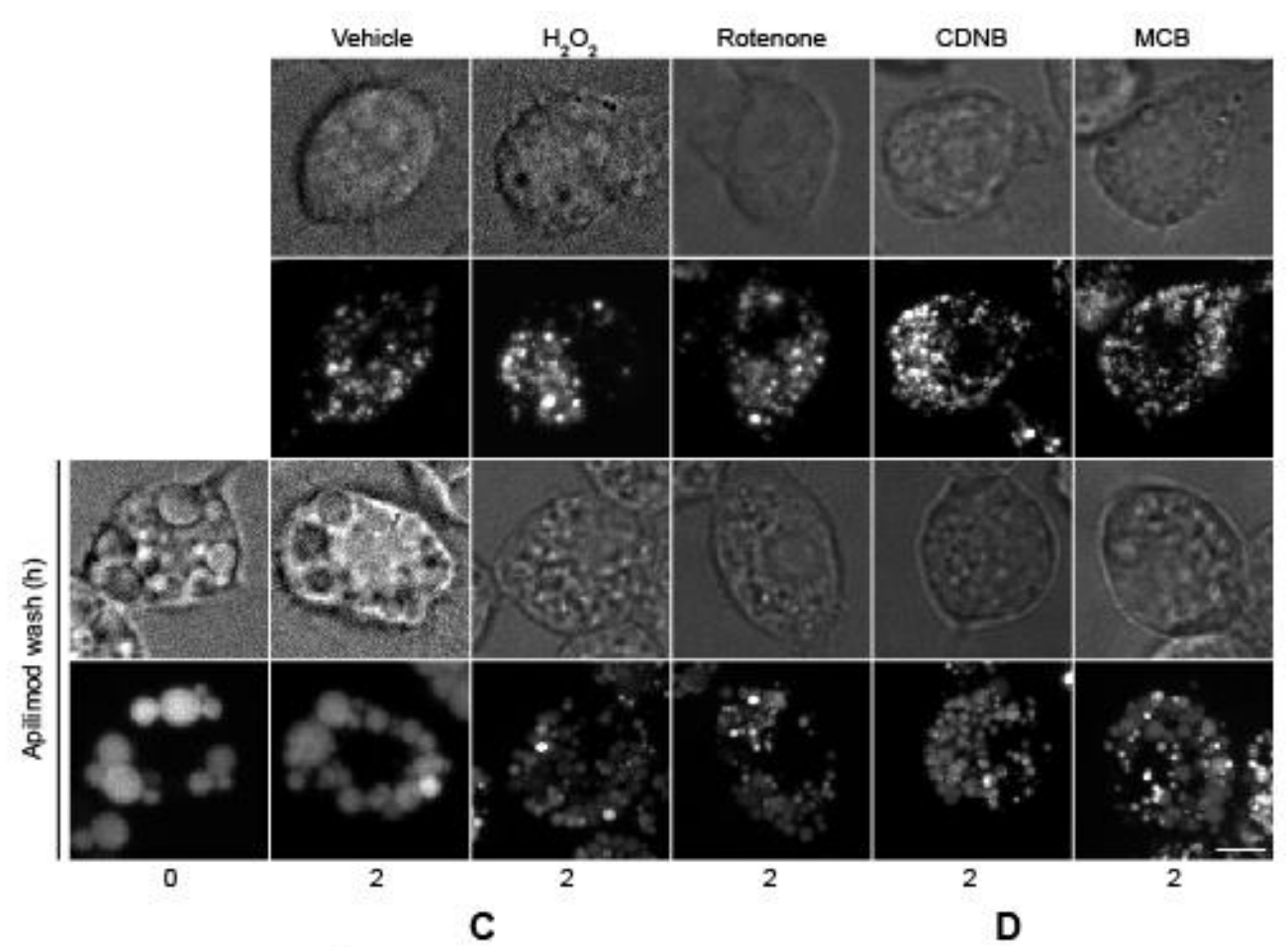

B

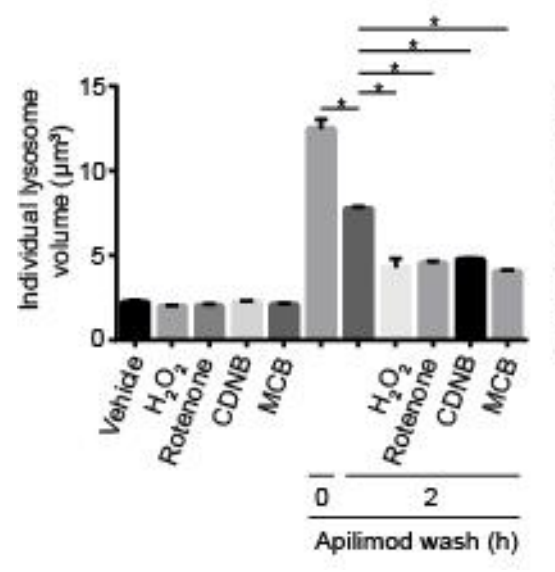

C

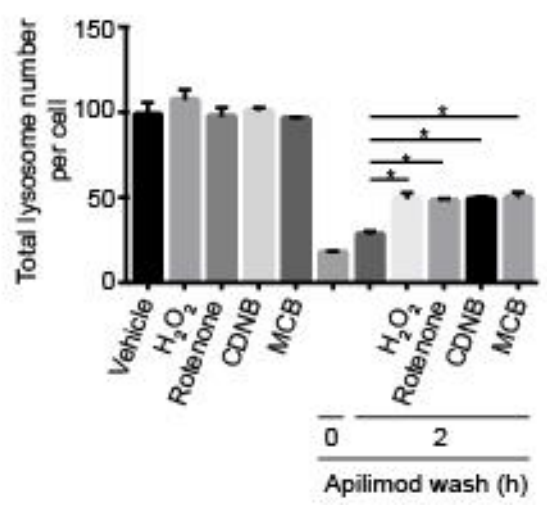

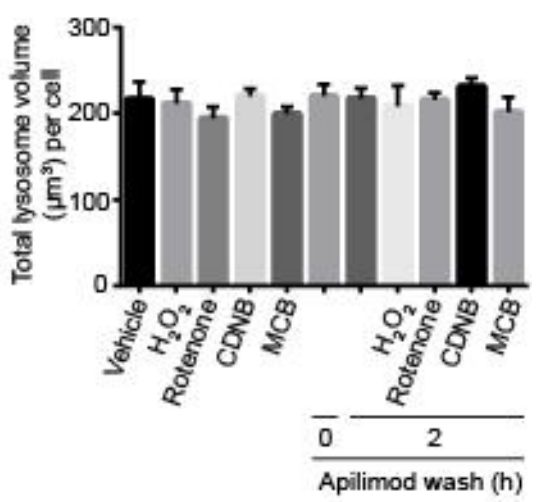

Figure 24. ROS accelerate recovery of lysosome size and number upon PIKfyve

reactivation. (A) Top two rows: RAW cells pre-labelled with Lucifer yellow were exposed to either vehicle, $1 \mathrm{mM} \mathrm{H} \mathrm{O}_{2} 40 \mathrm{~min}, 1 \mu \mathrm{M}$ rotenone $60 \mathrm{~min}, 10 \mu \mathrm{M}$ CDNB $30 \mathrm{~min}$, or $5 \mu \mathrm{M} \mathrm{MCB}$ 30 min. Bottom two rows: alternatively, RAW cells were first treated with $20 \mathrm{nM}$ apilimod for $60 \mathrm{~min}(0 \mathrm{~h})$, followed by apilimod removal and replenishment with complete media for $2 \mathrm{~h}$ in the presence of vehicle, $\mathrm{H}_{2} \mathrm{O}_{2}$, rotenone, $\mathrm{CDNB}$, or $\mathrm{MCB}$ at previously indicated concentrations. Fluorescence micrographs are spinning disc microscopy images with 45-55 z-planes represented 
as z-projections. Scale bar: $5 \mu \mathrm{m}$. (B-D) Quantification of individual lysosome volume (B), lysosome number per cell (C), and total lysosome volume per cell (D). Data are represented as mean \pm s.e.m. from three independent experiments, with $25-30$ cell assessed per treatment condition per experiment.

\subsubsection{ROS affect microtubule structure}

ROS have been reported to disrupt microtubule structure and we have shown previously that microtubules are necessary for lysosome coalescence during PIKfyve inhibition (Choy et al., 2018; Pocasap et al., 2018). We therefore examined microtubule structure in response to ROS for RAW cells (Fig. 25A) and RPE cells (Fig. 25B) by immunostaining against $\alpha$-tubulin. All four ROS agonists affected microtubule structure, however in distinct ways. Qualitative observations suggest $\mathrm{H}_{2} \mathrm{O}_{2}$ make microtubules more stable and extended, whereas rotenone, CDNB and MCB caused microtubules to be shorter and cytosolic diffused, indicative of microtubule depolymerisation, for both RAW cells (Fig. 25A) and RPE cells (Fig. 25B). These observations suggest different ROS species and/or sources of ROS synthesis regulate microtubule structure and possibly lysosome dynamics in distinct ways. We therefore set to inspect the impact of these four ROS agonists on lysosome movement. 

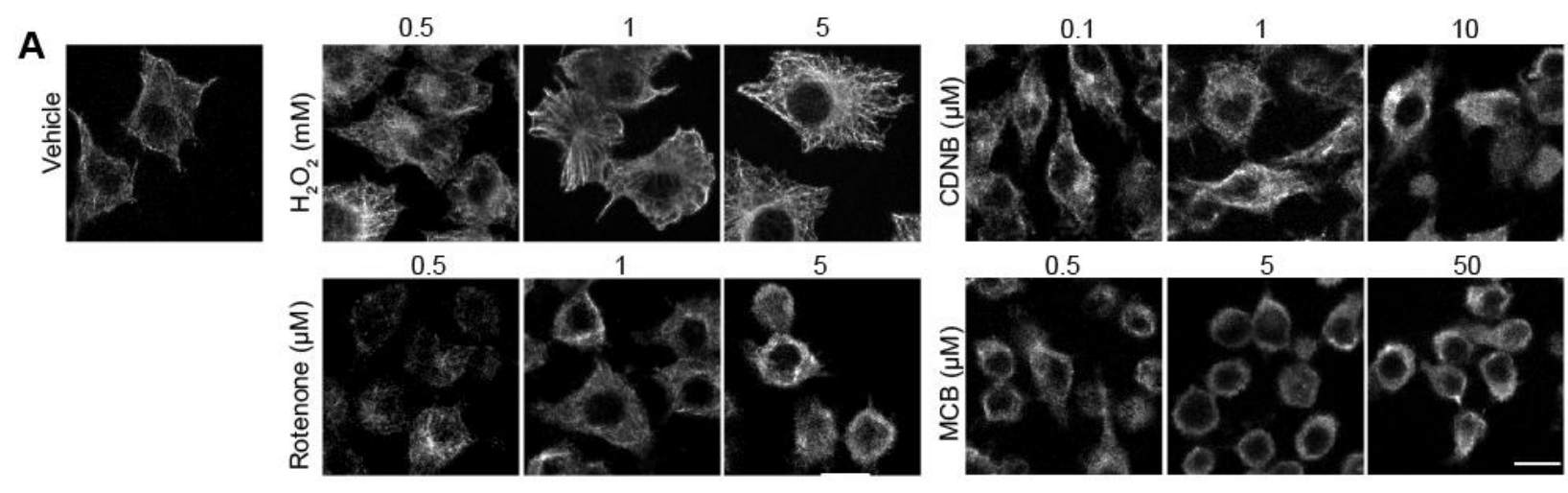

50

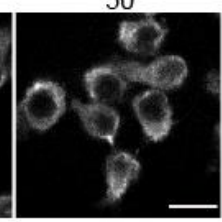

B
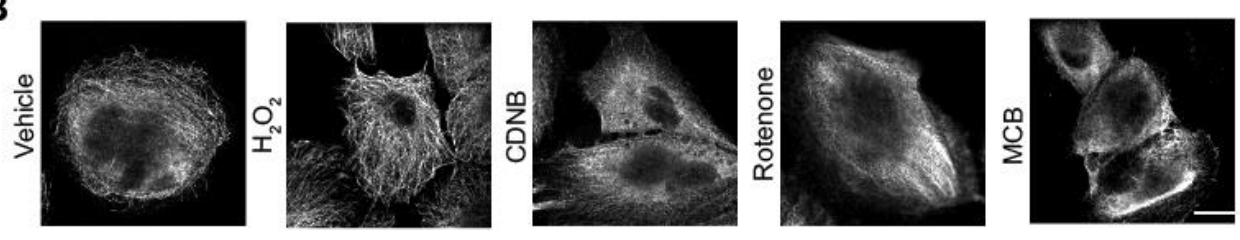

Figure 25. ROS agents differentially affect the microtubule system. Representative single zfocal plane immunofluorescence micrographs of RAW cells (A) or RPE cells (B) treated with vehicle, $\mathrm{H}_{2} \mathrm{O}_{2}$, rotenone, $\mathrm{CDNB}$ or $\mathrm{MCB}$ at previously used time periods and at the indicated concentrations. After treatment with ROS agents, cells were fixed and immunostained with anti$\alpha$-tubulin antibodies. Scale bar: $10 \mu \mathrm{m}$ (A) or $20 \mu \mathrm{m}$ (B).

\subsubsection{Distinct ROS regulation of lysosome movement}

To understand our observation of distinct microtubule structure regulation from different ROS agonists (Fig. 25), we monitored the effect of $\mathrm{H}_{2} \mathrm{O}_{2}$, rotenone, $\mathrm{CDNB}$, and $\mathrm{MCB}$, on lysosome motility indicators: lysosome speed, vectorial displacement, and track length. Following ROS stimulation with the four agonists, we tracked lysosome movement over 3 min for RAW cells (Fig. 26A-C), or 6 min for RPE cells (Fig. 26D-F). $\mathrm{H}_{2} \mathrm{O}_{2}$ was the only agonist that impaired all measures of lysosome motility indicators for RAW cells (Fig. 26A-C) and RPE cells 
(Fig. 26D-F). As microtubules appeared more stable and extended upon $\mathrm{H}_{2} \mathrm{O}_{2}$ application (Fig, 25), we tested to observe whether microtubule stability caused the cytoskeletal tracks to be frozen and impair lysosome mobility, using the microtubule stabilizing-drug paclitaxel (Weaver, 2014). However, paclitaxel failed to arrest lysosome coalescence from apilimod (Fig. 27A-D), and instead may increase lysosome movement (Fig. 27E-G). These observations suggest $\mathrm{H}_{2} \mathrm{O}_{2}$ arrest lysosome coalescence and movement through mechanisms distinct from affecting microtubule structure. This may occur due to displacement of microtubule motors, dynein and kinesin-1, from lysosome membranes. However, $\mathrm{H}_{2} \mathrm{O}_{2}$ did not seem to remove Arl8b GTPase or Rab7-GTP probe RILPC33, upstream effectors of kinesin-1 or dynein respectively, from lysosome membrane (Fig. 28).

Rotenone, $\mathrm{CDNB}$, and $\mathrm{MCB}$, compared to $\mathrm{H}_{2} \mathrm{O}_{2}$, did not affect lysosome movement indicators for RAW cells or RPE cells (Fig. 26). For RAW cells, rotenone, CDNB, and MCB caused cells to appear taller and rounder, causing a wobbling motion that moved lysosomes along with the cell, most likely due to microtubule depolymerisation. However, RPE cells were flatter and resistant to this wobbling effect. Since rotenone, CDNB, and MCB impaired microtubule structure (Fig. 25), we used the microtubule depolymerisation agent nocodazole, as a positive control (Blajeski et al., 2002). Nocodazole impaired lysosome mobility indicators, more drastically for RPE cell compared to RAW cells. Since nocodazole impaired lysosome movement and not rotenone, $\mathrm{CDNB}$, or $\mathrm{MCB}$ at the concentrations tested, this suggest that microtubule depolymerisation from these three ROS agonists are partial and not sufficient to explain inhibition of lysosome coalescence from apilimod. Therefore, we set to examine possible lysosome fission mechanisms stimulated by ROS to circumvent lysosome coalescence. 

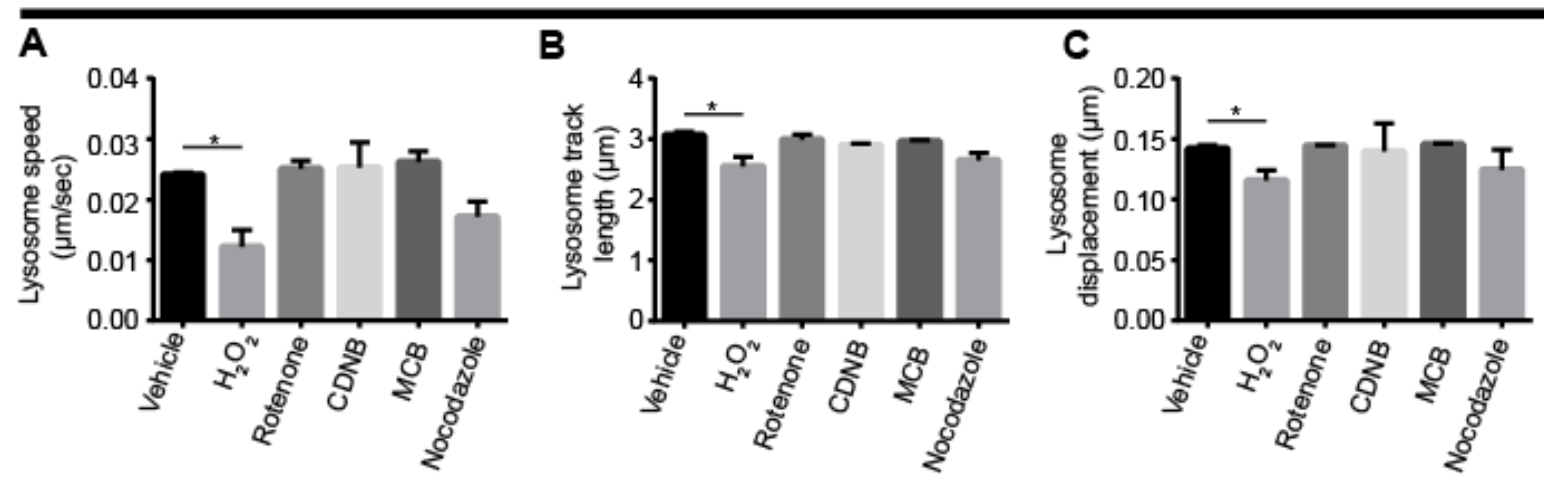

RPE cells
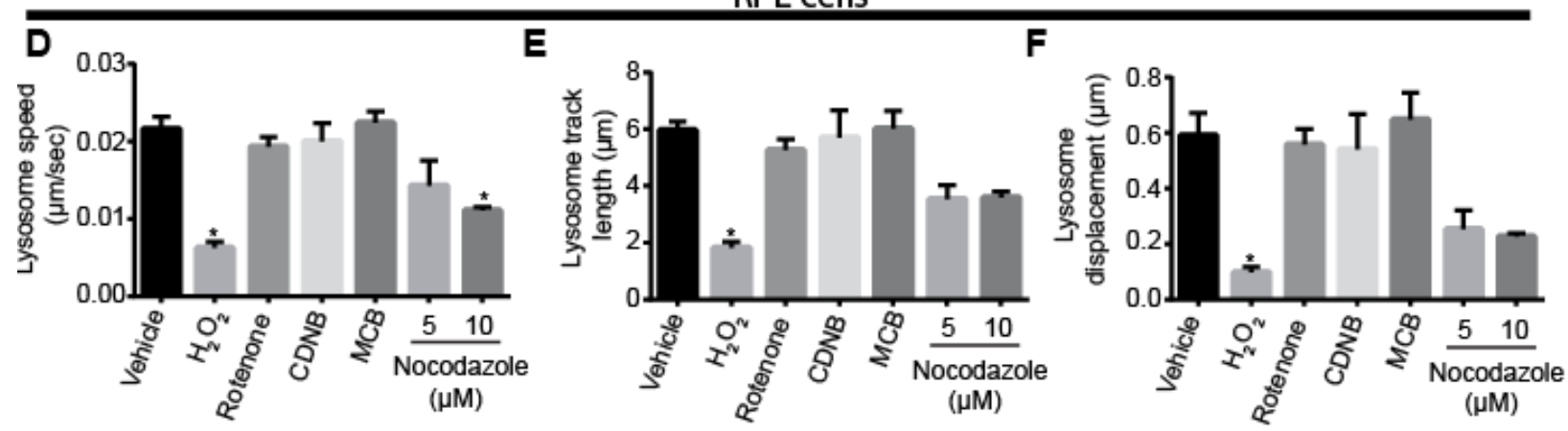

Figure 26: Distinct ROS agents differentially impact lysosome motility. RAW cells (A-C) or RPE cells (D-F) were pre-labelled with Lucifer yellow and exposed to either vehicle, $1 \mathrm{mM}$ $\mathrm{H}_{2} \mathrm{O}_{2} 40$ min, $1 \mu \mathrm{M}$ rotenone $60 \mathrm{~min}, 10 \mu \mathrm{M}$ CDNB $30 \mathrm{~min}, 5 \mu \mathrm{M}$ MCB $30 \mathrm{~min}$, or $5 \mu \mathrm{M}$ or 10 $\mu \mathrm{M}$ nocodazole for $60 \mathrm{~min}$. Live-cell spinning disc confocal microscopy was performed at a single, mid-cell z-focal plane once every $4 \mathrm{sec}$ for $3 \mathrm{~min}$ for RAW cells or every $8 \mathrm{sec}$ for $6 \mathrm{~min}$ for RPE cells. Automated quantification of lysosome speed (A, D), lysosome track length (B, E), and lysosome displacement $(\mathbf{C}, \mathbf{F})$ for RAW cells (A-C) or RPE cells (D-F). Data represented as mean \pm s.d. from three independent experiments. 
A

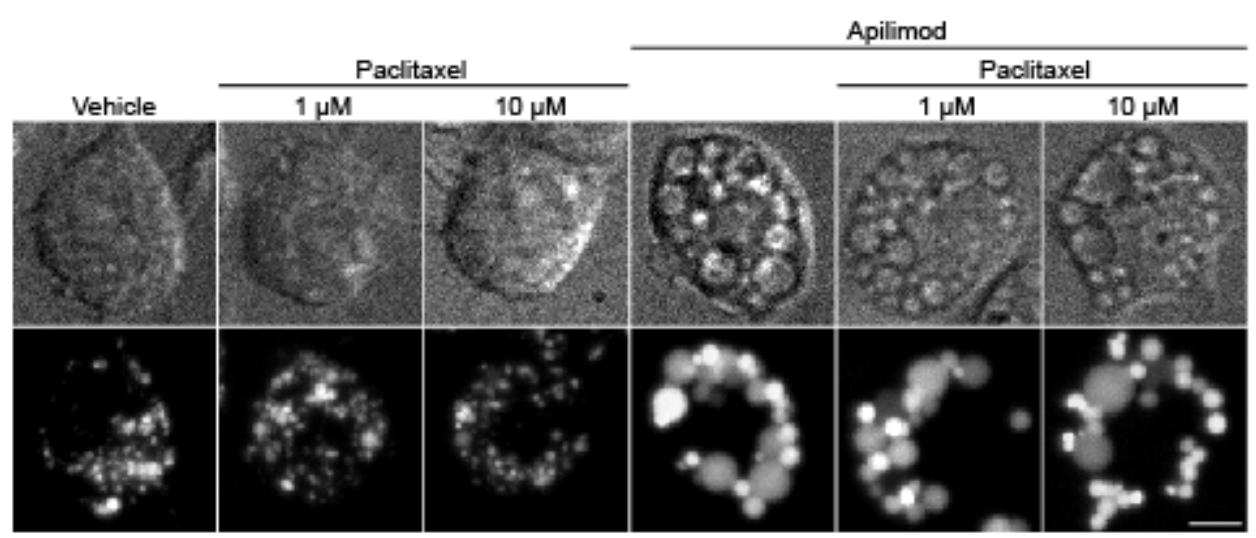

B

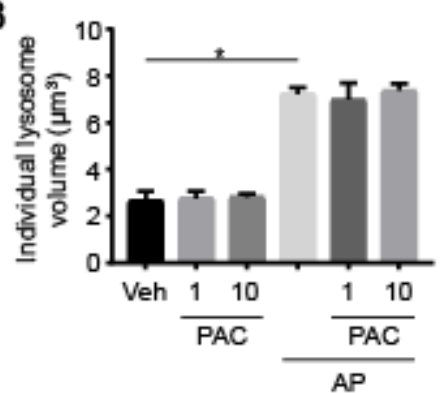

$\mathbf{E}$

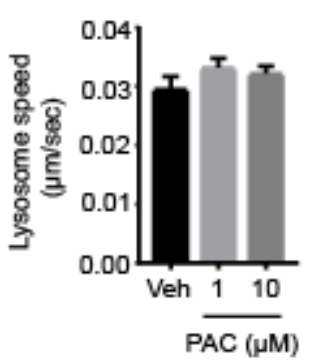

$\mathbf{F}$

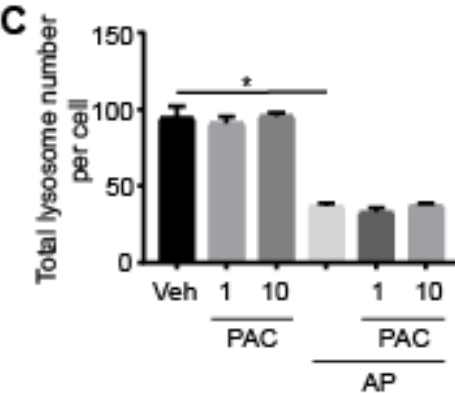

G
D

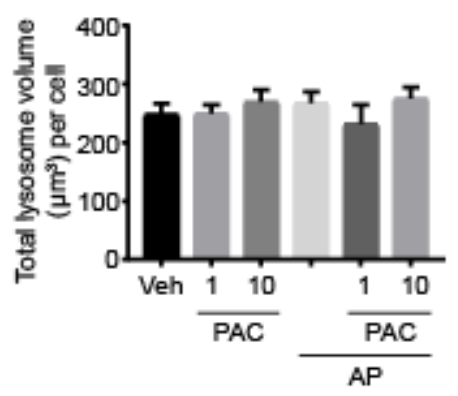

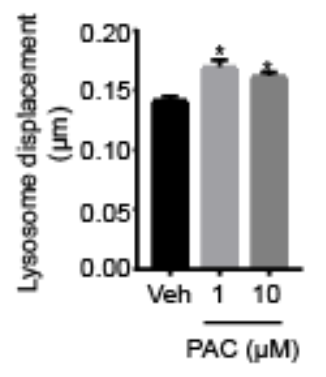

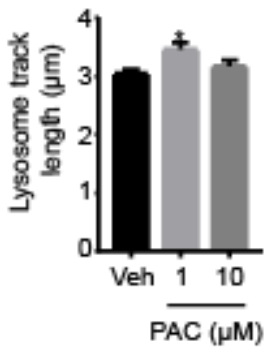

Figure 27: Increased microtubule stability does not affect lysosome motility or lysosome

coalescence during PIKfyve inhibition. (A) RAW cells pre-labelled with Lucifer yellow were exposed to either vehicle, or $1 \mu \mathrm{M}$ or $10 \mu \mathrm{M}$ paclitaxel for $60 \mathrm{~min}$ in presence or absence of 20 $\mathrm{nM}$ apilimod for the remaining $40 \mathrm{~min}$. Scale bar: $5 \mu \mathrm{m}$. (B-D) Quantification of individual lysosome volume (B), lysosome number per cell (C), and total lysosome volume per cell (D). Data are represented as mean \pm s.e.m. from three independent experiments, with 25-30 cell assessed for (B-D) per treatment condition per experiment. (E-G) RAW cells pre-labelled with Lucifer yellow were exposed to vehicle or $1 \mu \mathrm{M}$ or $10 \mu \mathrm{M}$ paclitaxel 60 min. Live cell spinning 
disc confocal microscopy was performed at single z-focal plane once every 4 sec for 3 min.

Quantification of lysosome speed (E), lysosome displacement (F), and lysosome track length (G) are shown. Data are represented as \pm s.d. from three independent experiments.
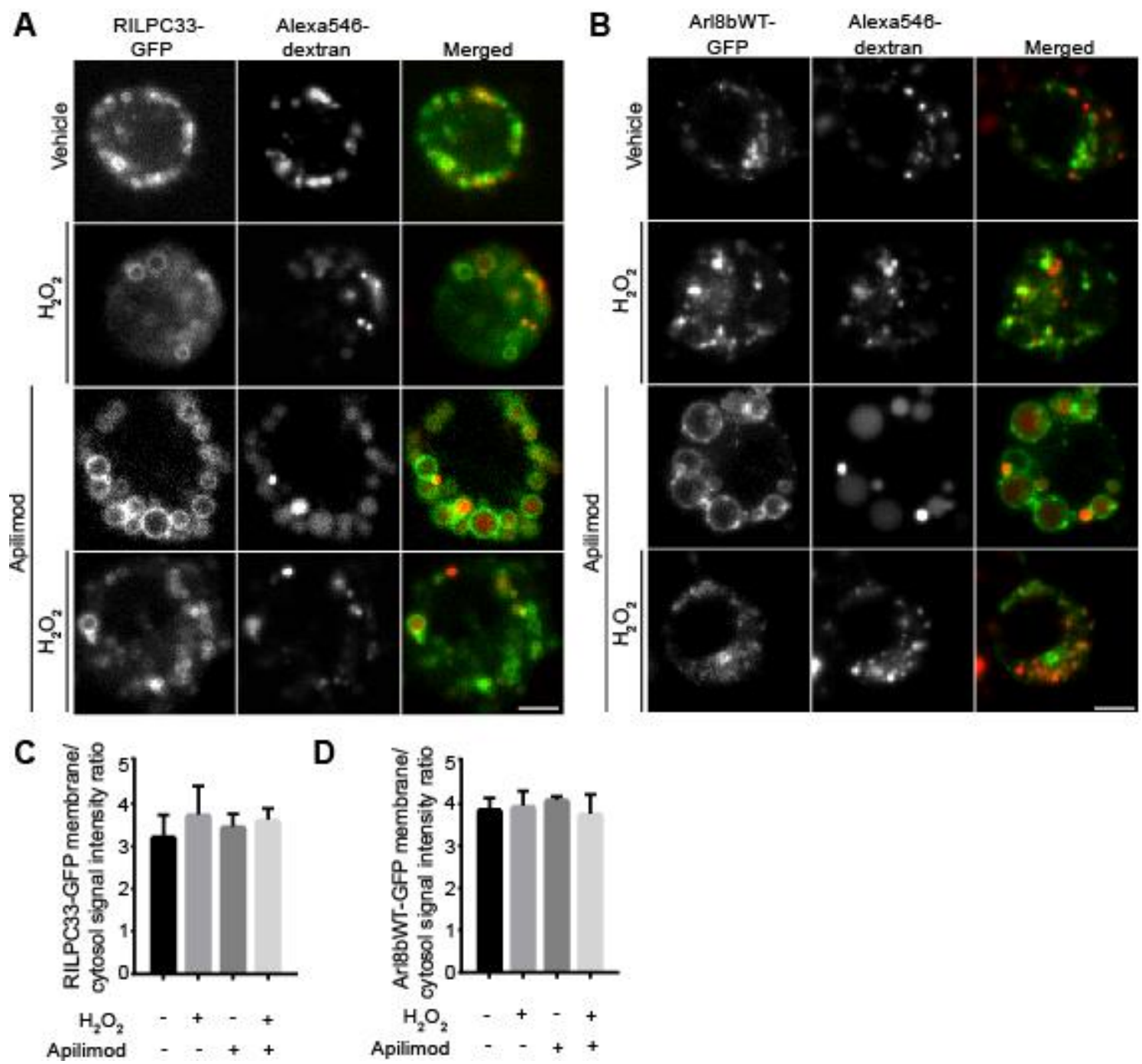

Figure 28. ROS do not affect Rab7 activation and Arl8b loading onto lysosomes. RAW cells expressing RILPC33-GFP (A), or Arl8bWT-GFP (B), exposed to vehicle in absence or presence of $20 \mathrm{nM}$ apilimod $40 \mathrm{~min}$, or $1 \mathrm{mM} \mathrm{H}_{2} \mathrm{O}_{2} 40 \mathrm{~min}$ in presence or absence of $20 \mathrm{nM}$ apilimod 40 min. Scale bar: $5 \mu \mathrm{m}$. (C-D) Quantification of membrane associated fluorescence intensity of 
RILPC33-GFP (C) from (A) or Arl8bWT-GFP (D) from (B), normalized to cytosol fluorescence intensity. Data represent mean \pm s.e.m. from three independent experiments, with 15-20 cell assessed per treatment condition per experiment.

\subsubsection{ROS does not stimulate lysosome fission through clathrin or dynamin}

Clathrin and dynamin are part of canonical fission machinery that assemble on lysosomes and autolysosomes (Boutry et al., 2018; Rong et al., 2012; Saffi and Botelho, 2019). We therefore explored the possibility of ROS stimulating clathrin and/or dynamin recruitment on lysosomes to boost lysosome fission to prevent coalescence from apilimod. We used clathrineGFP expressing RPE cells and showed that although there was no difference in clathrin recruitment on Alexa ${ }^{546}$-conjugated dextran positive lysosomes for vehicle or apilimod treatment, $\mathrm{H}_{2} \mathrm{O}_{2}$ in presence or absence of apilimod increased clathrin association on lysosomes (Fig. 29A). Complementary to this observation, we performed sucrose gradient ultracentrifugation and organelle fractionation, and demonstrated increased clathrin and dynamin-2 recruitment to LAMP1 positive lysosome fractions for $\mathrm{H}_{2} \mathrm{O}_{2}$ treated RAW cells compared to vehicle treatment (Fig. 29B-D). To correlate $\mathrm{H}_{2} \mathrm{O}_{2}$-dependent clathrin and dynamin2 recruitment to possible lysosome fission, we tested to observe that whether upon apilimod removal and lysosome fragmentation, clathrin or dynamin inhibition could prevent accelerated lysosome fragmentation by $\mathrm{H}_{2} \mathrm{O}_{2}$. However, we failed to distinguish lysosome size and number between treatment conditions of $\mathrm{H}_{2} \mathrm{O}_{2}$ application during apilimod removal in absence or presence of clathrin inhibition through ikarugamycin (Fig. 30), or dynamin inhibition through dyngo-4A (Fig. 31) (Elkin et al., 2016; McCluskey et al., 2013). Lysosome fragmentation was similarly unaffected for treatment conditions of rotenone application in presence or absence of 
dyngo-4A during apilimod removal (Fig. 31). Therefore, although $\mathrm{H}_{2} \mathrm{O}_{2}$ increase canonical fission machinery components clathrin and dynamin on lysosome membrane, clathrin and dynamin are not involved in $\mathrm{H}_{2} \mathrm{O}_{2}$-dependent lysosome fragmentation to prevent coalescence from apilimod.

A

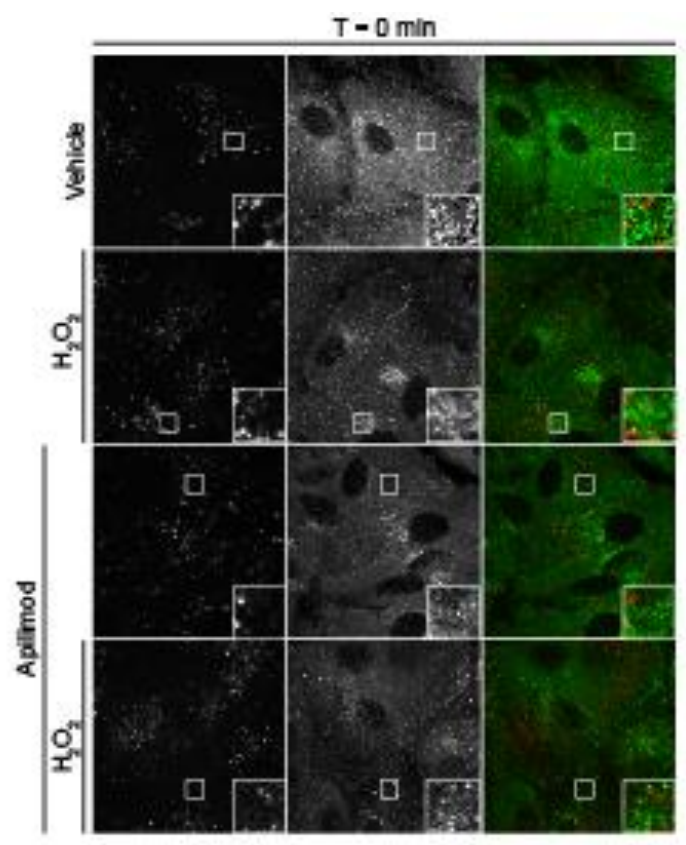

B

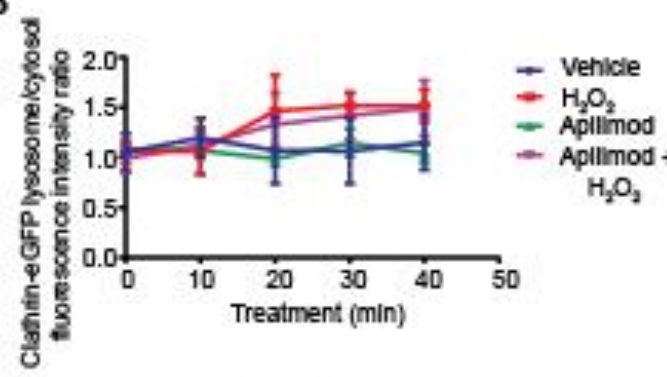

$\mathrm{C}$

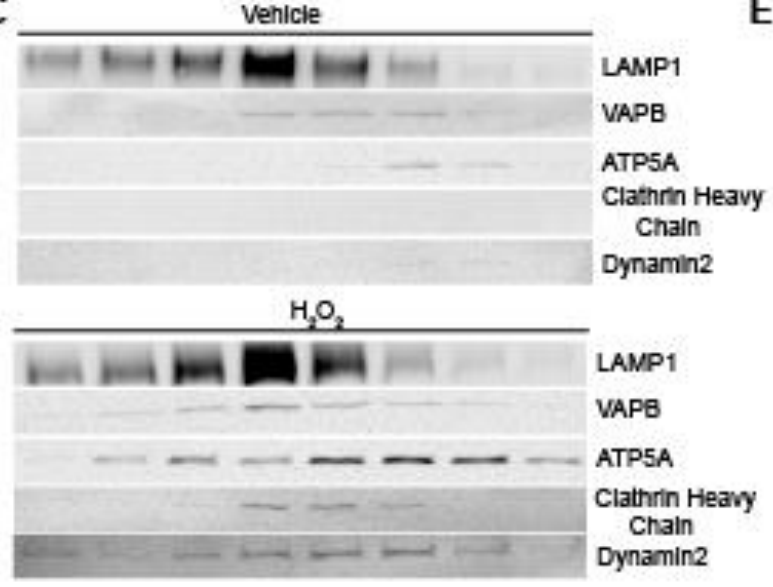

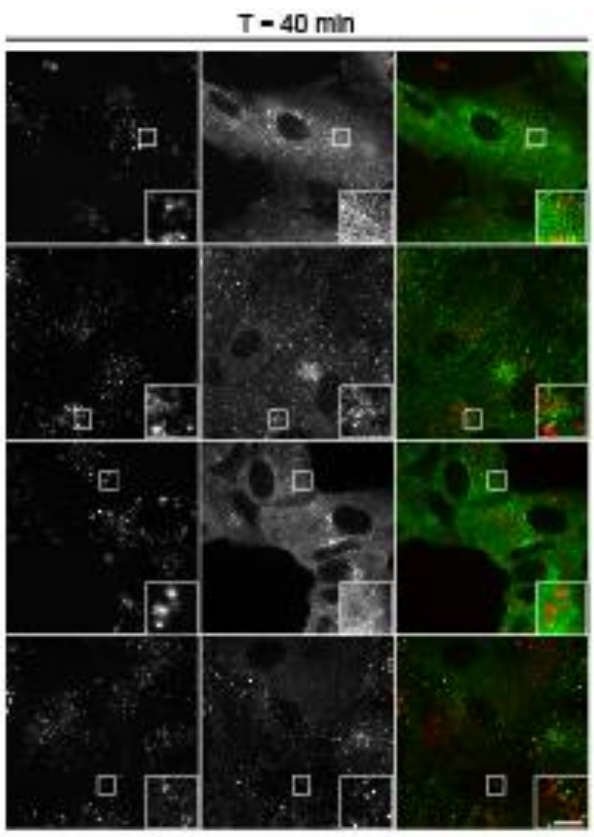

D

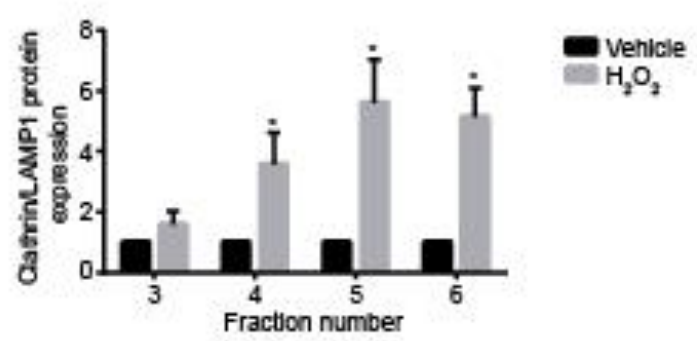

E

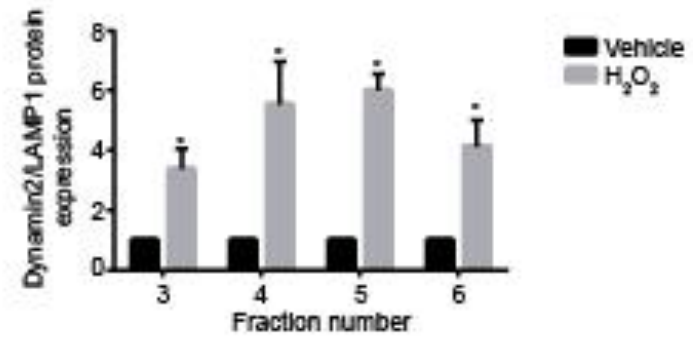


Figure 29. $\mathrm{H}_{2} \mathrm{O}_{2}$ boosts recruitment of clathrin and dynamin to membranes. (A) RPE cells stably expressing clathrin heavy chain-eGFP were pre-labelled with Alexa ${ }^{546-}$ conjugated dextran

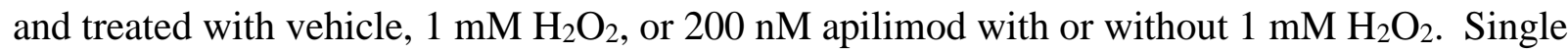
z- plane images were acquired every 2 min for 40 min across all treatments. Fluorescence micrographs represent single z-plane images at $0 \mathrm{~min}$ and $40 \mathrm{~min}$ for each treatment obtained by spinning disc microscopy. The inset is a magnified portion of field of view tracking dextran ${ }^{546}$ conjugated dextran lysosome(s) or clathrin-eGFP separate or merged. Scale bar: $7 \mu \mathrm{m}$. (B) Ratio of clathrin-eGFP fluorescence intensities associated with Alexa ${ }^{546}$-conjugated dextran structures to cytosol at time points: $0,10,20,30$ and $40 \mathrm{~min}$. Data are represented as mean \pm s.e.m. from five to six independent experiments, with 1-3 cells assessed per treatment condition per experiment. Two-way ANOVA and Tukey's post-hoc test were used for $(\mathbf{B})$, where * indicates $P<0.05$ against control conditions. (C) RAW cells were treated with vehicle or $1 \mathrm{mM} \mathrm{H}_{2} \mathrm{O}_{2}$ for $40 \mathrm{~min}$, lysed and homogenates fractionated through a sucrose gradient ultracentrifugation. Fractions were immunoblotted against LAMP1 and VAPB to respectively identify lysosome and ER fractions, and against clathrin heavy chain and dynamin 2. Protein expression for clathrin heavy chain (D) or dynamin 2 (E) were normalized to LAMP1 for fractions 3 to 6. Data are represented as \pm s.d. from three independent experiments. 


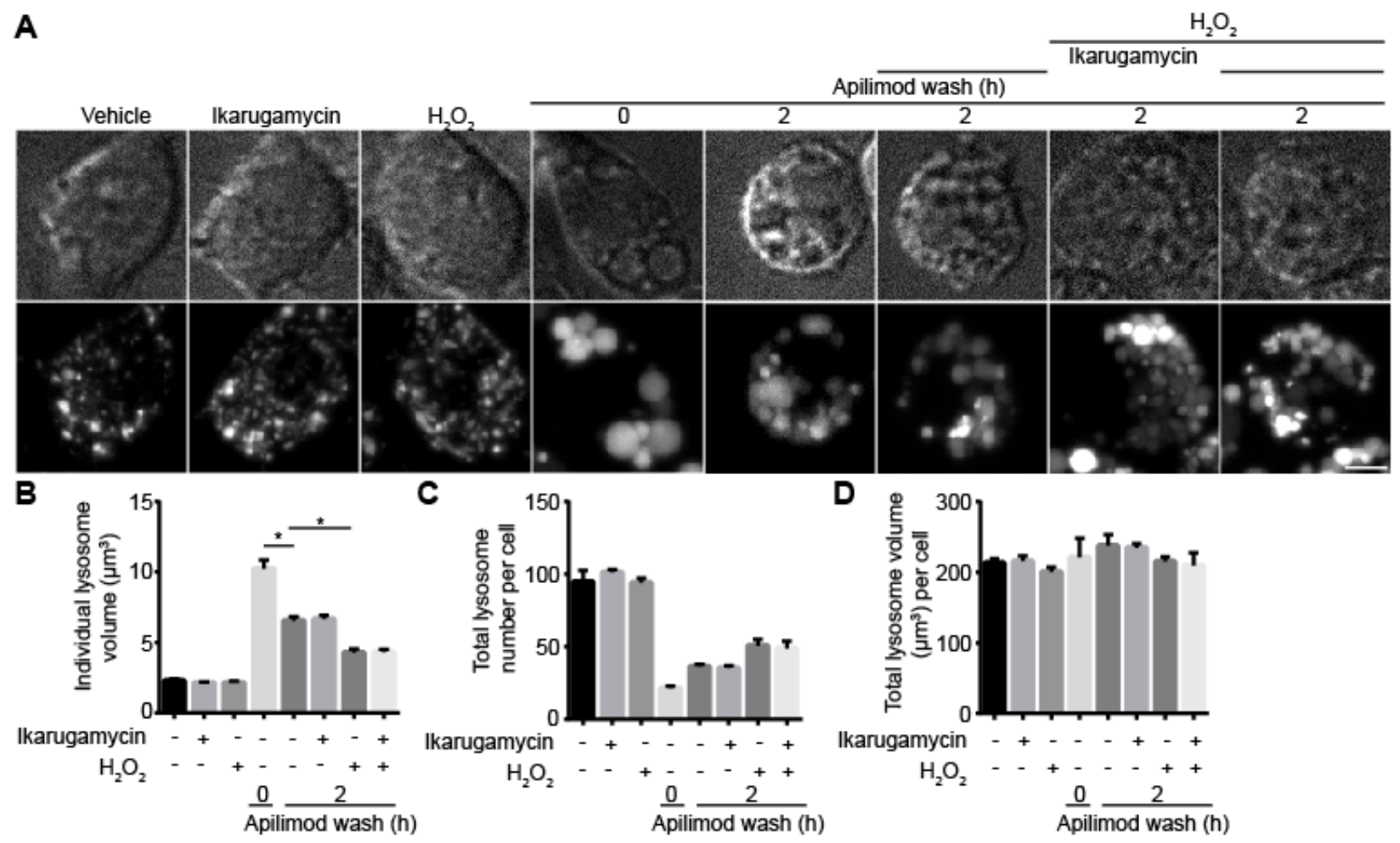

Figure 30: Clathrin inhibition does not arrest ROS-mediated lysosome fragmentation

during PIKfyve reactivation. (A) RAW cells were pre-labelled with Lucifer yellow and exposed to either vehicle alone, $1 \mathrm{mM} \mathrm{H}_{2} \mathrm{O}_{2}$ for $40 \mathrm{~min}, 1 \mu \mathrm{M}$ ikarugamcyin for $1 \mathrm{~h}$, or $20 \mathrm{nM}$ apilimod for 60 min. For a subgroup of cells treated with apilimod, drug was replaced with fresh media containing either vehicle, $1 \mathrm{mM} \mathrm{H}_{2} \mathrm{O}_{2}, 1 \mu \mathrm{M}$ ikarugamcyin, or $1 \mathrm{mM} \mathrm{H}_{2} \mathrm{O}_{2}$ and $1 \mu \mathrm{M}$ ikarugamycin for $2 \mathrm{~h}$. Fluorescence micrographs are spinning disc microscopy images with 4555 z-planes represented as z-projections. Scale bar: $5 \mu \mathrm{m}$. (B-D) Quantification of individual lysosome volume (B), lysosome number per cell (C), and total lysosome volume per cell (D). Data shown as mean \pm s.e.m. from three independent experiments, with 25-30 cell assessed per treatment condition per experiment. 
A

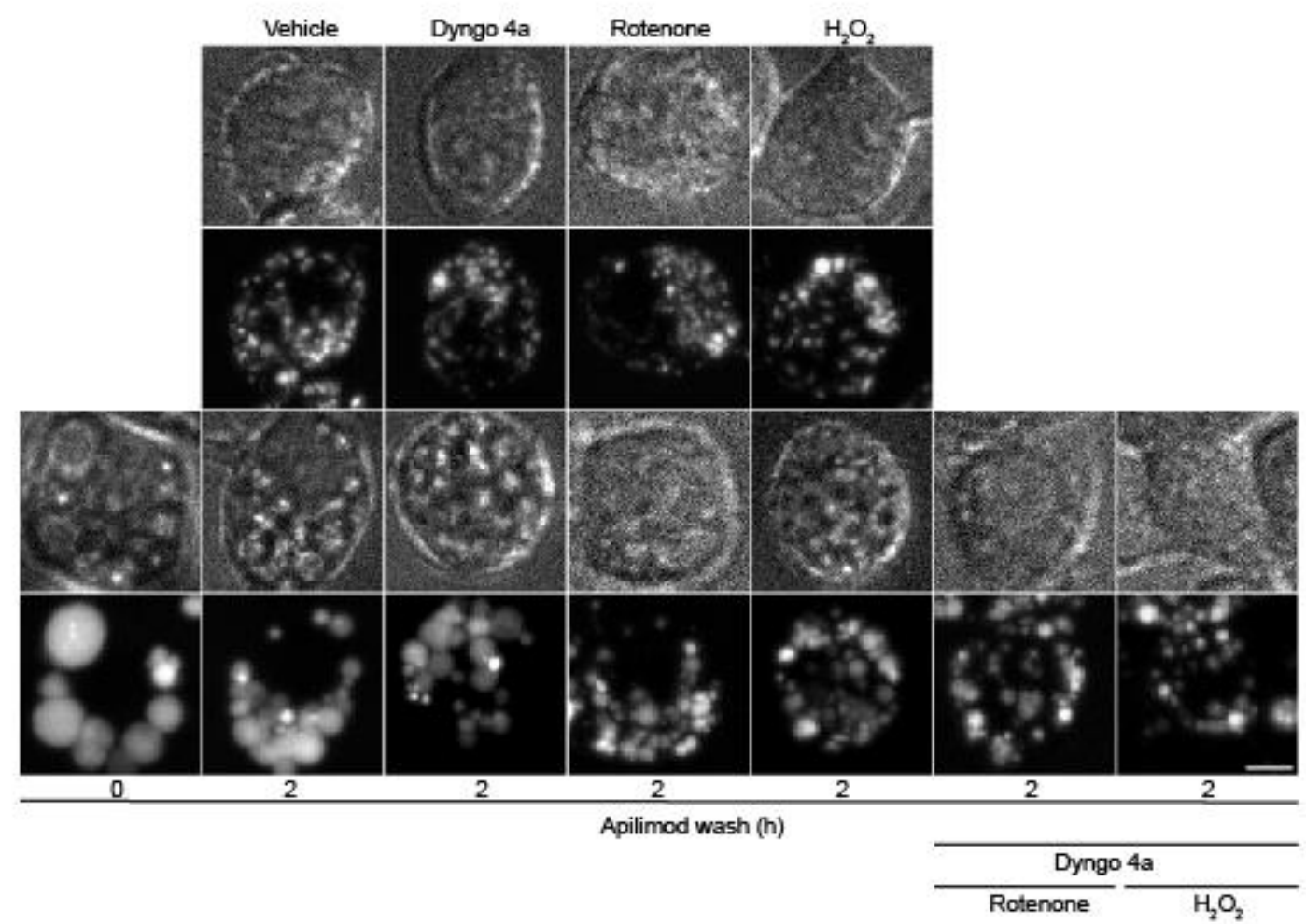

B

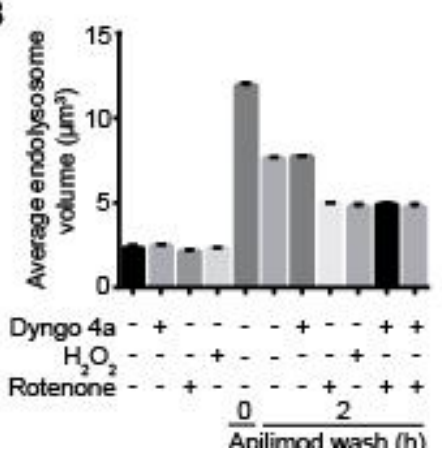

C

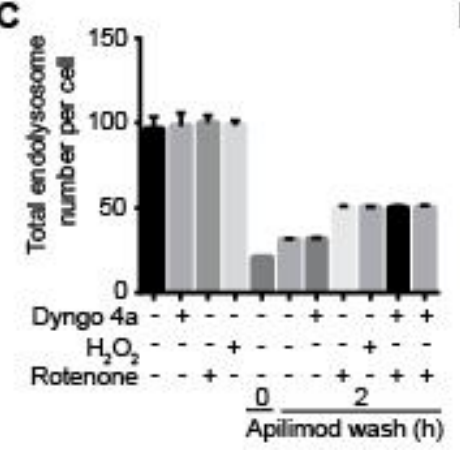

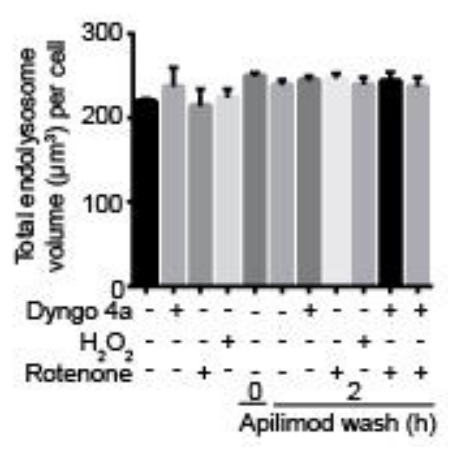

Figure 31. Dynamin inhibition does not affect lysosome fragmentation during during

PIKfyve reactivation. (A) RAW cells were pre-labelled with Lucifer yellow and exposed to either vehicle, $30 \mu \mathrm{M}$ dyngo-4A for $2 \mathrm{~h}, 1 \mathrm{mM} \mathrm{H}_{2} \mathrm{O}_{2}$ for $40 \mathrm{~min}$, or $1 \mu \mathrm{M}$ rotenone for $1 \mathrm{~h}$, or 20 $\mathrm{nM}$ apilimod for $60 \mathrm{~min}$. Additional subgroup of apilimod treated cells were then washed and incubated with apilimod-free media and changed for $2 \mathrm{~h}$ in the presence of vehicle, dyngo-4A, $\mathrm{H} 2 \mathrm{O} 2$, and dyngo4-A plus $\mathrm{H} 2 \mathrm{O} 2$ for a total time of $2 \mathrm{~h}$ without apilimod. Fluorescence micrographs are spinning disc microscopy images with 45-55 z-planes represented as z- 
projections. Scale bar: $5 \mu \mathrm{m}$. (B-D) Quantification of individual lysosome volume (B), lysosome number per cell $(\mathbf{C})$, and total lysosome volume per cell (D). Data is illustrated as mean \pm s.e.m. from three independent experiments, with 25-30 cell assessed per treatment condition per experiment.

\subsubsection{ROS remove actin from lysosomes to prevent coalescence}

PtdIns(3,5) $\mathrm{P}_{2}$ depletion enhance actin nucleation, resulting in actin accumulation on endosomes and melanosomes to prevent fission for enlargement (Bissig et al., 2019; Hasegawa et al., 2016). We thus considered and examined actin structures on lysosomes upon PtdIns(3,5) $\mathrm{P}_{2}$ depletion. Indeed, acute PIKfyve inhibition through apilimod increased number of punctate phalloidin stained actin structures on Alexa ${ }^{488}$-conjugated dextran lysosomes (Fig. 32A, B). ROS may modify actin structure for perturbation and clearance through modification of actin cytosolic cysteine and methionine residues (Wilson and González-Billault, 2015). ROS stimulated actin clearance on lysosomes may allow fission to occur to prevent coalescence from acute PIKfyve inhibition. CDNB or rotenone administration with apilimod reduced number of lysosome associated actin punctate structures compared to apilimod treatment (Fig. 32A, B). To evaluate whether actin clearance prevent lysosome coalescence from acute PIKfyve inhibition, we applied latrunculin A or cytochalasin D to depolymerise actin during apilimod treatment and apilimod removal. Apilimod administration induced lysosome enlargement by increasing individual lysosome volume and decreasing lysosome number, and cytochalasin D or latrunculin A coadministration resisted apilimod induced lysosome enlargement. Conversely, apilimod removal increased lysosome fragmentation by reducing individual lysosome volume and increasing lysosome number, and administration of cytochalasin D or latrunculin A during apilimod 
removal further augmented lysosome fragmentation (Fig. 32C-F). Our observations suggest ROS promote actin clearance on lysosomes. Furthermore, actin depolymerisation prevents lysosome coalescence during apilimod treatment and increases lysosome fragmentation during apilimod removal. Therefore, ROS through actin clearance may promote lysosome fission to prevent coalescence during acute PIKfyve inhibition. 

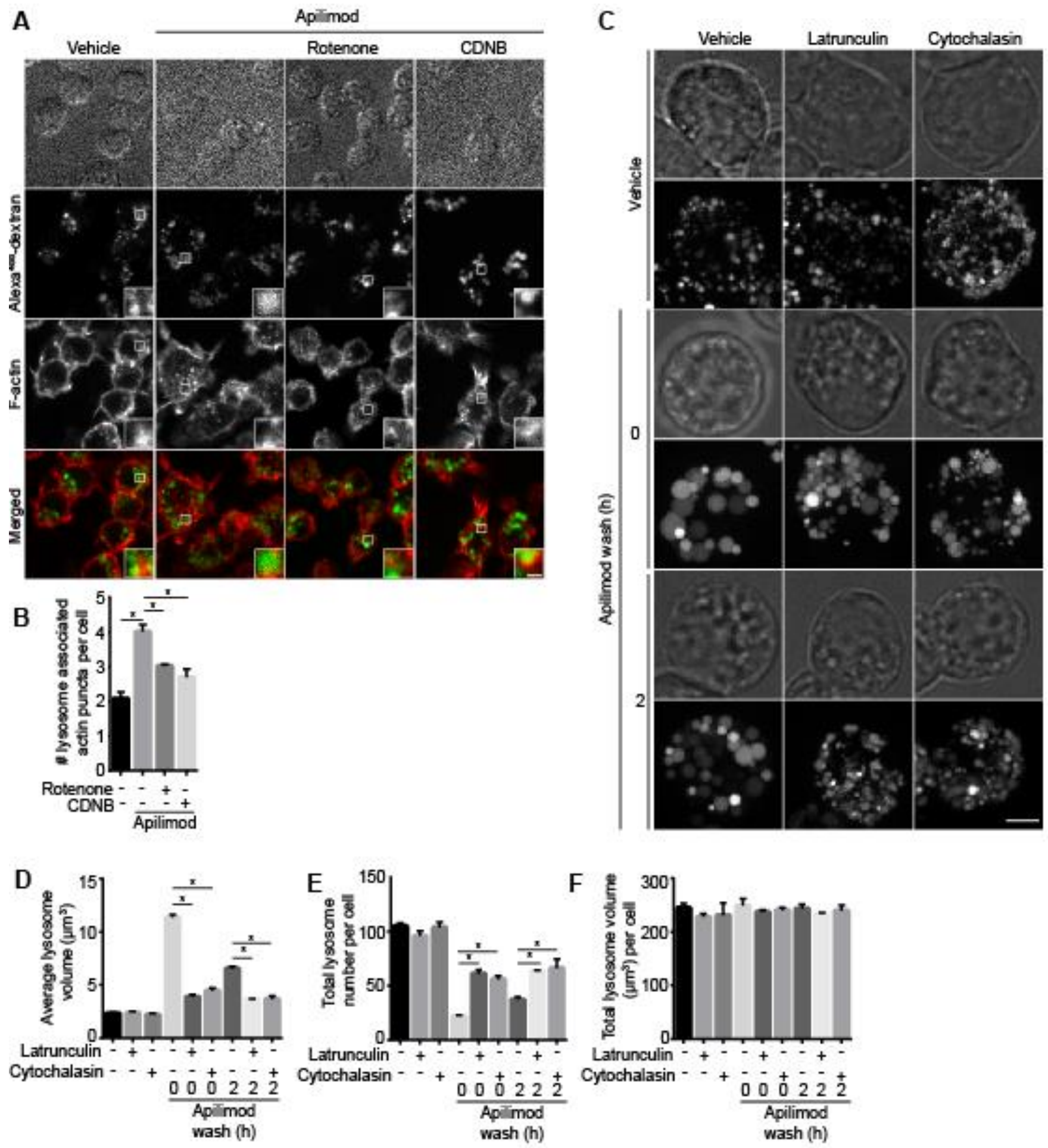

Figure 32: ROS promote actin clearance to rescue lysosome coalescence during PIKfyve inhibition and accelerate fragmentation during PIKfyve reactivation. (A) RAW cells prelabelled with Alexa ${ }^{488}$-conjugated dextran followed by treatment with vehicle, $20 \mathrm{nM}$ apilimod 
$40 \mathrm{~min}$ alone, or in presence of $10 \mu \mathrm{M}$ CDNB $30 \mathrm{~min}$ or $1 \mu \mathrm{M}$ rotenone $60 \mathrm{~min}$. Cells fixed with 4\% PFA and stained for actin with phalloidin. Fluorescence micrographs are spinning disc images with single z-focal plane. The inset is a magnified portion of field of view tracking dextran ${ }^{488}$-conjugated dextran lysosome(s) or phalloidin-stained actin separate or merged. Scale bar: $2 \mu \mathrm{m}$. (B) Cells assessed for number of actin puncta structures associated with lysosomes per cell from (A). Data represent mean \pm S.E.M. from three independent experiments, with $60-80$ cells assessed per treatment condition across three experiments. One-way ANOVA and Tukey's post-hoc test was used, where * indicates statistical significance between indicated conditions $(p<0.05)$. (C) RAW cells pre-labelled with Lucifer yellow and exposed to vehicle or $20 \mathrm{nM}$ apilimod for $1 \mathrm{~h}$ followed by apilimod removal for $0 \mathrm{~h}$ or $2 \mathrm{~h}$. These conditions were then supplemented with additional vehicle or $1 \mu \mathrm{M}$ latrunculin $\mathrm{A}$ for $1 \mathrm{~h}$, or $5 \mu \mathrm{M}$ cytochalasin $\mathrm{D}$ for $1 \mathrm{~h}$. Fluorescence micrographs are represented as z-projections of 45-55 z-plane images obtained by spinning disc microscopy. Scale bar: $5 \mu \mathrm{m}$. $D-F$ : Quantification of individual lysosome volume (D), lysosome number per cell (E), and total lysosome volume per cell (F). Data represent mean \pm S.E.M. from three independent experiments, with 25-30 cells assessed per treatment condition per experiment. 


\section{Chapter 5: Discussion}




\subsection{Lysosome enlargement due to inhibition of the lipid kinase PIKfyve occurs through coalescence}

Lysosome membranes consist of signalling phosphoinositide lipids that regulate traffic and lysosome morphology. $\operatorname{Ptd} \operatorname{Ins}(3,5) \mathrm{P}_{2}$ serves as a signaling lipid on the lysosome membrane to regulate retrograde traffic, endocytic traffic, phagosome maturation, autophagic flux, ion channel activity, actin assembly, and lysosome size (Bissig et al., 2017; Hasegawa et al., 2016; Ho et al.,

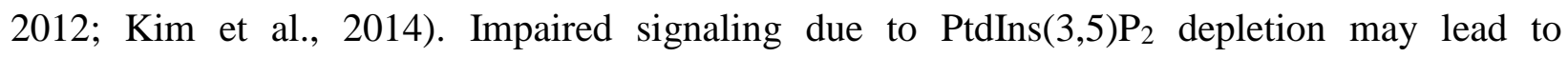
physiological aberrations such as neurodegeneration, embryogenic lethality, inflammation, glucose intolerance, and abnormal storage of proteins and lipids with enlarged lysosomes (Ikonomov et al., 2007; Ikonomov et al., 2016; Mironova et al., 2016; Shisheva, 2008). The most visibly dramatic phenotype from $\operatorname{PtdIns}(3,5) \mathrm{P}_{2}$ depletion is lysosome enlargement. Such dysregulation of lysosome homeostasis remains poorly characterized in terms of how $\operatorname{PtdIns}(3,5) \mathrm{P}_{2}$ regulates lysosome size and number. Therefore, we presented the hypothesis that $\operatorname{PtdIns}(3,5) \mathrm{P}_{2}$ depletion enlarges lysosomes by increasing individual lysosome volume while simultaneously either reducing lysosome number through "coalescence" resulting in total lysosome volume per cell being unaffected, and/or unchanged lysosome number through lysosome "dilation" to increase total lysosome volume per cell. To understand the impact of $\operatorname{PtdIns}(3,5) \mathrm{P}_{2}$ depletion and synthesis on lysosome size and number, we employed quantitative 3D Volocity image analysis software, and set exclusion criteria for identifying labelled lysosomes and automated lysosome volume and number analysis. 


\subsubsection{Lysosomes coalesce from PtdIns(3,5) $P_{2}$ depletion due to disrupted lysosome fusion- fission cycle}

Our results with quantitative image analysis demonstrate that lysosomes enlarge upon acute and genetic PtdIns(3,5) $\mathrm{P}_{2}$ depletion due to increase in individual lysosome volume and decrease in lysosome number, while keeping total lysosome volume constant. This suggests that lysosomes enlarge via coalescence. Such lysosome coalescence is reversible upon PIKfyve re-activation following PIKfyve inhibition due to lysosomes splitting and fragmenting, causing decrease in individual lysosome volume while increasing number, thus suggesting a role of $\operatorname{Ptd} \operatorname{Ins}(3,5) \mathrm{P}_{2}$ synthesis from PIKfyve re-activation in lysosome fragmentation or "fission". This is supported through sweptfield confocal imaging, employing low powered laser light as excitation source coupled with slit that scan the laser across entire image plane to excite fluorophores without inducing photo-toxicity, which revealed that upon acute PIKfyve inhibition, lysosome "splitting" or "fission" was impaired. Thus, lysosomes enlarge during $\operatorname{PtdIns}(3,5) \mathrm{P}_{2}$ depletion by "coalescence" caused by impaired lysosome "splitting" under continuous lysosome fusion.

\subsubsection{Microtubules regulate PtdIns(3,5) $P_{2}$ dependent lysosome fusion-fission}

Lysosomes are constantly in a dynamic flux, undergoing fusion and fission events. Whether during full-fusion or through kiss-and-run, the interactions between lysosomes and other organelles, including other lysosomes, depends on microtubule-dependent motors (Bissig et al., 2017; Saffi and Botelho, 2019; Storrie and Desjardins, 1996). Indeed, we demonstrated that lysosome enlargement from acute PIKfyve inhibition is dependent on microtubules and associated motor protein complexes, dynein and kinesin-1. Alternatively, microtubules regulate lysosome 
fragmentation or "shrinkage" by augmenting fragmentation upon microtubule depolyermisation during PIKfyve re-activation. Microtubules serve as cytoskeletal tracks to position lysosomes for interaction and coalescence upon acute PIKfyve inhibition, and removal of the tracks or inhibition of the motor complexes prevent lysosome coalescence likely by blocking fusion (Fig. 33).

\subsubsection{Lysosome coalescence from acute PIKfyve inhibition is independent of protein biosynthesis}

Acute PIKfyve inhibition causes accumulation of transcription factor EB (TFEB) within the nucleus, and TFEB binds to CLEAR (Coordinated Lysosome Expression and Regulation) DNA sequences to increase expression of genes leading to lysosome and autophagosome biogenesis, autophagosome maturation and turnover, and lysosome exocytosis. In addition to acute PIKfyve inhibition, mTORC1 inhibition also causes nuclear translocation of TFEB due to inhibited phosphorylation at Serine 122, Serine 142, Serine 211, Serine 332 and Serine 402 residues, thereby releasing TFEB from the lysosome membrane (Napolitano and Ballabio, 2016; Settembre et al., 2011; Vega-Rubin-de-Celis et al., 2017; Wang et al., 2015). To understand the role of TFEB and whether lysosome biogenesis contributes to lysosome volume increase during acute PIKfyve inhibition due to additional protein input, I collaborated with Dr. Christopher H. Choy and Dr. Matthew Gray, to investigate such possibility, which I elaborate below. 


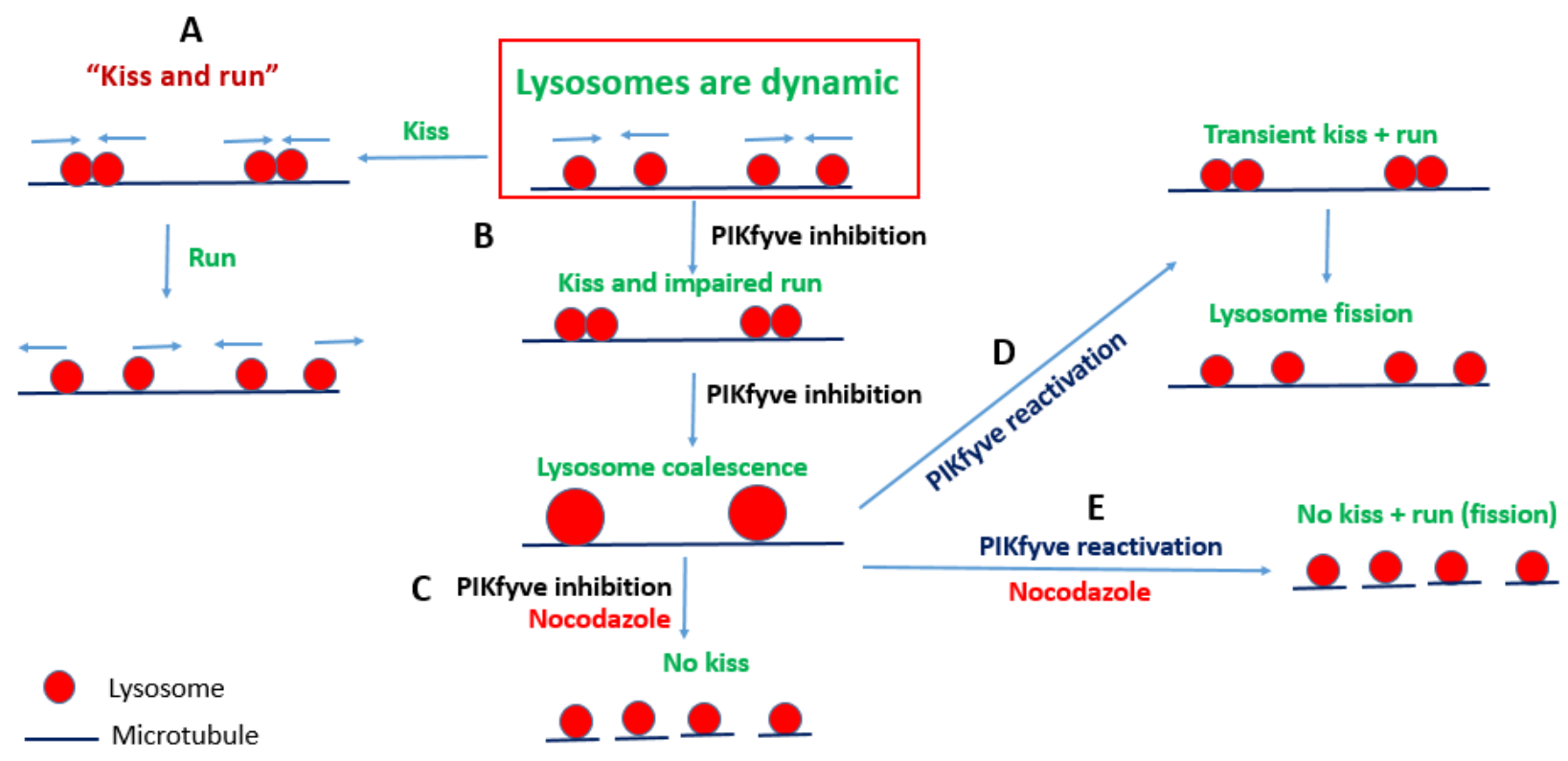

Figure 33: Microtubule depolymerisation affect lysosome "kiss-and-run" during PIKfyve inhibition and reactivation. A) Lysosomes interact through microtubule-dependent transient interaction ("kiss") followed by separation ("run"). B) During PIKfyve inhibition, lysosomes collide (kiss) but do not separate (run) properly, causing lysosome coalescence. C) Microtubule depolymerisation through nocodazole during PIKfyve inhibition removes microtubule tracks for lysosome interaction, thus preventing coalescence. D) Following PIKfyve inhibition and lysosome coalescence, PIKfyve reactivation causes lysosomes to interact (kiss) through microtubules and separate (run) frequently. Since, lysosome splitting/separation occurs more frequently, the dynamic is favored towards lysosome fragmentation or "fission". E) Depolymerising microtubules during PIKfyve reactivation prevent lysosome interaction (kiss), thus favoring the dynamic more towards lysosome splitting or "fission" compared to PIKfyve reactivation. 


\subsubsection{TFEB activation from acute PIKfyve inhibition does not contribute to lysosome coalescence}

Work by Drs. Choy and Gray determined that TFEB localizes to the nucleus upon acute PIKfyve inhibition, independently of mTORC1 (Supp. Fig. 1 and 2). This increased the expression of lysosome genes for selected genes that we tested, including LAMP1, MCOLN1, cathepsin D and V-ATPase H and D subunits (Supp. Fig. 3). However, while mRNA levels increased, this did not lead to a corresponding boost in protein expression (Supp. Fig. 3). PtdIns(3,5) $\mathrm{P}_{2}$ depletion impair traffic of proteins to lysosomes, which may prevent proper assembly of proteins at the lysosome to impair lysosome protein turnover (Sharma et al., 2019). Depletion of TFEB or related transcription factors TFE3 (Supp. Fig. 4), or alternatively protein synthesis inhibition did not arrest lysosome enlargement upon acute PIKfyve inhibition (Supp. Fig. 4), suggesting a lack of requirement of lysosome gene expression or protein input for lysosome enlargement. Therefore, although acute PIKfyve inhibition causes mTORC1-independent nuclear translocation of TFEB and increases lysosome gene expression, such phenotype is independent and does not contribute to lysosome volume during enlargement (Choy et al., 2018).

Depletion of PtdIns(3,5) $\mathrm{P}_{2}$ enlarges lysosomes with abnormal storage of proteins and lipids within lumen due to defective recycling of membrane and luminal contents through lysosome reformation or "fission" from endolysosomes (Bissig et al., 2017; Mironova et al., 2016). Our research adds to current literature by providing increasingly conclusive evidence regarding the role of PtdIns $(3,5) \mathrm{P}_{2}$ in maintaining lysosome number and size during repeated microtubule-dependent lysosome fusion-fission cycles. Additionally, we also show independence and dissociation of lysosome enlargement from PIKfyve-suppressed TFEB nuclear translocation. 


\subsubsection{Future directions}

Although from our study, we provide evidence for the role of $\operatorname{Ptd} \operatorname{Ins}(3,5) \mathrm{P}_{2}$ in regulating lysosome fission during lysosome fusion-fission cycling, there is lack of understanding regarding the mechanism for how such fission occurs. PtdIns(3,5) $\mathrm{P}_{2}$ interacts with Atg18, and Atg18 regulates retrograde traffic from the vacuole/lysosome in yeast; possibly this occurs because Atg18 can induce membrane tubulation and scission as demonstrated with recombinant Atg18 using unilamellar vesicles (Gopaldass et al., 2017). However, in mammals, it is unclear if Atg18 orthologs can do the same. It is yet to be characterized if related PROPPINs WIPI-1 to -4 localize to lysosome membrane in mammalian cells and whether such localization can induce lysosome membrane reformation possibly through $\operatorname{PtdIns}(3,5) \mathrm{P}_{2}$ interaction with the WIPI proteins. Though, the mammalian homologue of Atg18, WIPI-2, localizes to autophagosomes, it is not known if this induces fission from autophagosomes (Polson et al., 2010). If indeed WIPI proteins are involved in lysosome fission, siRNA targeted depletion would enlarge and/or possibly tubulate lysosomes. Dependence between PtdIns(3,5) $\mathrm{P}_{2}$ and WIPI could be assessed through observing whether PtdIns(3,5) $\mathrm{P}_{2}$ depletion through apilimod remove WIPI from lysosomes, and if indeed apilimod removal and PtdIns(3,5) $\mathrm{P}_{2}$ synthesis can localize WIPI to the lysosome. To further address requirement of PtdIns(3,5) $\mathrm{P}_{2}$ for WIPI-dependent membrane fission, unilamellar vesicles with different phosphoinositides could be used and assessed for membrane curvature and scission for PtdIns $(3,5) \mathrm{P}_{2}$ rich vesicles. To understand site of WIPI membrane fission, WIPI proteins could be tracked for localization at neck of lysosome membrane tubules and/or membrane buds as possible sites for constriction.

PtdIns $(3,5) \mathrm{P}_{2}$ regulates actin nucleation through competitive interaction with actin for cortactin; consequently, PtdIns(3,5) $\mathrm{P}_{2}$ depletion forms actin aggregates around endosomes and 
melanosomes that appears to prevent endosome fission, possibly resulting in coalescence (Bissig et al., 2019; Hasegawa et al., 2016). It would be highly informative if future work could address whether PtdIns(3,5) $\mathrm{P}_{2}$ depletion accumulates actin aggregates around lysosomes, and whether actin clearance through actin depolymerisation and/or $\operatorname{Ptd} \operatorname{Ins}(3,5) \mathrm{P}_{2}$ synthesis can rescue lysosome coalescence.

From our observations, acute PIKfyve inhibition increases lysosome gene expression through TFEB nuclear translocation, without affecting corresponding protein expression. Additionally, TFEB is dispensable for lysosome enlargement. We have yet to observe whether the magnitude of such gene expression and possible protein expression is reproducible or perhaps greater in genetic and chronic models of $\operatorname{Ptd} \operatorname{Ins}(3,5) \mathrm{P}_{2}$ depletion by deleting genes encoding for PIKfyve, ArPIKfyve/Vac14, and/or Sac3/Fig4. If magnitude of protein expression is higher in genetic models of PtdIns(3,5) $\mathrm{P}_{2}$ depletion compared to our studies, this would suggest a possible threshold of signaling caused by PIKfyve loss that needs to be bypassed in order to translate changes in gene expression into protein synthesis. Our study only examined genes for lysosome proteins. It would be significant to add to our current knowledge if we could carry out RNA-seq studies that can functionally categorize genes that are affected by PtdIns(3,5) $\mathrm{P}_{2}$ depletion using wild-type and TFEB-deleted cells, which exist and are available. This would provide insight regarding possible group of genes that may regulate protein synthesis or turnover controlled by PtdIns(3,5) $\mathrm{P}_{2}$ and TFEB and even other yet to be discovered processes regulated by $\operatorname{PtdIns}(3,5) \mathrm{P}_{2}$. 


\subsection{ROS regulate lysosome fusion-fission dynamics during PIKfyve inhibition}

ROS include superoxide, hydrogen peroxide $\left(\mathrm{H}_{2} \mathrm{O}_{2}\right)$, and the hydroxyl radical with oxidizing potential to perturb cellular structures, such as proteins, lipids and DNA (Bibov et al., 2018; Schieber and Chandel, 2014). Additionally, they can act as localized signaling molecules for lysosomes by activating the lysosome-associated TRPML1 calcium channel and transcription factor EB (TFEB) for lysosome biogenesis, regulating autophagosome-lysosome fusion, and may regulate microtubules and actin structure (Wilson and González-Billault, 2015; Zhang et al., 2016; Zheng et al., 2016). Microtubules and actin associated with lysosomes may be regulated by ROS for affecting lysosome fusion and fission dynamics. We have demonstrated that lysosomes enlarge through coalescence and impaired fission when cells are depleted of $\operatorname{PtdIns}(3,5) \mathrm{P}_{2}$. Upon attempting to visually capture lysosome enlargement through conventional fluorescence spinning disc confocal microscopy, we noticed arrest of lysosome enlargement in cells exposed to frequent laser excitation due to phototoxicity. Microscopy based photodamage can induce ROS production (Icha et al., 2017). This observation led to the hypothesis that ROS regulate lysosome fusion and/or fission mechanisms and that this can rescue lysosome coalescence during acute PIKfyve inhibition.

To understand whether ROS regulate lysosome fusion-fission dynamics during acute PIKfyve inhibition, we employed four different ROS agonists: $\mathrm{H}_{2} \mathrm{O}_{2}$, rotenone for impairing mitochondrial electron transport chain (ETC) complex 1, CDNB for inhibiting thioredoxin reductase, and MCB for depleting glutathione activity (Heinz et al., 2017; Kamencic et al., 2000; Padgaonkar et al., 2015). $\mathrm{H}_{2} \mathrm{O}_{2}$ possess oxidizing potential and is a precursor for the hydroxyl radical, whereas rotenone, $\mathrm{CDNB}$, and $\mathrm{MCB}$ all produce superoxide, which can then decompose to $\mathrm{H}_{2} \mathrm{O}_{2}$ and hydroxyl radical (Fig. 8). We tested these four agonists producing different ROS on 
their effect on lysosome enlargement during acute PIKfyve inhibition and lysosome fragmentation during PIKfyve re-activation.

\subsubsection{ROS prevent lysosome enlargement during acute PIKfyve inhibition and accelerate fragmentation during PIKfyve re-activation}

All four agonists, $\mathrm{H}_{2} \mathrm{O}_{2}$, rotenone, $\mathrm{CDNB}$, and $\mathrm{MCB}$, prevented lysosome enlargement during acute PIKfyve inhibition and accelerated lysosome fragmentation during PIKfyve reactivation. Previous observations suggest that $\operatorname{Ptd} \operatorname{Ins}(3,5) \mathrm{P}_{2}$ synthesis stimulates lysosome fission (Choy et al., 2018). One possibility to explain these observations was that ROS can modify protein and lipid phosphatase active site through perturbation; thus, ROS may inactivate Fig4 phosphatase and enhance PtdIns(3,5) $\mathrm{P}_{2}$ levels (Schieber and Chandel, 2014). However, we tested and failed to observe a boost in PtdIns(3,5) $\mathrm{P}_{2}$ levels in response to $\mathrm{H}_{2} \mathrm{O}_{2}$ or rotenone. Therefore, ROS do not seem to increase PtdIns(3,5) $\mathrm{P}_{2}$ levels to prevent lysosome coalescence during acute PIKfyve inhibition.

ROS can affect actin and microtubule structure through oxidation of cytosolic cysteine SH sulfhydryl and methionine -SC residues (Wilson and González-Billault, 2015). We thus attempted to observe whether the four ROS agonists regulate cytoskeletal structure and whether such regulation can control lysosome fusion-fission dynamics. However, we noticed that depending on the ROS species produced and/or site of ROS synthesis by the four ROS agonists, cytoskeletal structure and lysosome dynamics were affected distinctly. 


\subsubsection{ROS affect lysosome fusion-fission dynamics distinctly}

\subsubsection{1 $\mathrm{H}_{2} \mathrm{O}_{2}$ effect on lysosome enlargement and fragmentation may occur by disrupting lysosome motility}

$\mathrm{H}_{2} \mathrm{O}_{2}$ enhanced microtubule structure by making them more stable and extended. However, this might not be a generalizable effect and may differ depending on cell type and/or treatment conditions. For example, human osteosarcoma cells display increased monomeric $\alpha$-tubulin and reduced polymerized tubulin upon $\mathrm{H}_{2} \mathrm{O}_{2}$ application. However, $\mathrm{HeLa}$ cells treated with $\mathrm{H}_{2} \mathrm{O}_{2}$ increased microtubule acetylation, indicator of microtubule stability (LEE et al., 2005; Mackeh et al., 2014). Additionally, $\mathrm{H}_{2} \mathrm{O}_{2}$ impaired lysosome mobility. However, microtubule stabilization is not sufficient to explain $\mathrm{H}_{2} \mathrm{O}_{2}$ impairment of lysosome movement and inhibition of coalescence since paclitaxel failed to arrest apilimod mediated lysosome enlargement and instead enhanced lysosome mobility. $\mathrm{H}_{2} \mathrm{O}_{2}$ may instead regulate lysosome dynamics through microtubule motor complexes, dynein and kinesin-1. For example, $\mathrm{H}_{2} \mathrm{O}_{2}$ activates the MAP kinase $\mathrm{p} 38 \alpha$, and $\mathrm{p} 38 \alpha$ through phosphorylation of microtubule-associated kinesin-1 impairs motor function on mitochondria, and such impaired mobility is rescued upon forced anchorage of kinesin-1 on mitochondria. Forced anchorage of kinesin-1 on mitochondria achieved through kinesin-1 expressing HA-Kif5B-FRB and mitochondrial targeting TOM20-mCherry-FKBP, and FKBPFRPB hetero-dimerization through rapamycin (Debattisti et al., 2017; Morfini et al., 2013). Thus, $\mathrm{H}_{2} \mathrm{O}_{2}$ may regulate RILP-ORP1L-dynein, FYCO1-dynein, p38 $\alpha$-kinesin-1, and/or SKIP-kinesin1 complexes, although not likely through lysosome associated upstream effectors Rab7-GTP or Arl8b-GTPase for RILP or SKIP respectively because loading of these effectors onto lysosome membranes was unaffected through $\mathrm{H}_{2} \mathrm{O}_{2}$ application in presence or absence of apilimod.. 
Collectively, we determined that $\mathrm{H}_{2} \mathrm{O}_{2}$ likely impairs lysosome mobility, preventing lysosome coalescence during acute PIKfyve inhibition.

$\mathrm{H}_{2} \mathrm{O}_{2}$ enhanced recruitment of canonical fission machinery components, clathrin and dynamin on lysosomes. However, $\mathrm{H}_{2} \mathrm{O}_{2}$ does not require clathrin or dynamin for lysosome fission because inhibition of these components during apilimod removal did not prevent accelerated lysosome fragmentation induced by $\mathrm{H}_{2} \mathrm{O}_{2} \cdot \mathrm{H}_{2} \mathrm{O}_{2}$ may co-ordinate lysosome fission through multiple components, of which clathrin or dynamin activity may be dispensable, and maybe instead part of a complex circuit. Below, I will discuss future directions to better delineate these concepts.

\subsubsection{Rotenone, CDNB and MCB effect on lysosome enlargement and fragmentation}

Compared to $\mathrm{H}_{2} \mathrm{O}_{2}$ that produces hydroxyl radical, rotenone, CDNB, and MCB produce superoxide, which is decomposed to $\mathrm{H}_{2} \mathrm{O}_{2}$ and hydroxyl radical, through inhibition of mitochondrial ETC complex 1, disrupting thioredoxin activity and depleting glutathione, respectively. Although MCB target thiols in glutathione, MCB may also impair thiol function on enzymes such as thioredoxin reductase to produce superoxide (Kamencic et al., 2000; Lubos et al., 2011; Nordberg et al., 1998). Rotenone, CDNB, and MCB partially depolymerised microtubules without significantly affecting lysosome mobility. Although RAW cells displayed a wobbling effect due to microtubule depolymerisation, causing the lysosomes to move in bulk within the cell, such observation was not reproducible in RPE cells, which are flatter and resistant to this oscillation. Since microtubule depolymerisation by nocodazole impaired lysosome movement, 
partial microtubule depolymerisation is not sufficient to explain how lysosomes resist enlargement from apilimod in response to these three ROS agonists.

Instead, our data suggest that ROS produced from rotenone, CDNB and MCB may affect actin structure to promote lysosome fission. $\operatorname{PtdIns}(3,5) \mathrm{P}_{2}$ promotes actin turnover, and PtdIns(3,5) $\mathrm{P}_{2}$ depletion amasses F-actin on endosomes and lysosomes (endosome marker Rab7 found on lysosomes), thereby hindering fission and leading to coalescence. Specifically, PtdIns $(3,5) \mathrm{P}_{2}$ competes with F-actin for interaction with cortactin, involved in actin nucleation, and upon PtdIns $(3,5) \mathrm{P}_{2}$ depletion, cortactin nucleate and enrich actin on endosomes and lysosomes that disrupt membrane scission (Bissig et al., 2019; Hong et al., 2015). Indeed, CDNB and rotenone removed lysosome associated F-actin structures that amassed upon Ptdins $(3,5) \mathrm{P}_{2}$ depletion. Therefore, CDNB and rotenone promote actin clearance from lysosomes to promote fission and prevent lysosome enlargement from acute PIKfyve inhibition. MCB was not tested for effect on actin structure due to time constraints. Additionally, actin depolymerisation prevented lysosome enlargement during apilimod treatment and accelerated lysosome fragmentation upon apilimod removal, further suggesting a role for actin clearance in preventing lysosome coalescence and accelerating lysosome fission. Overall, our observations suggest that ROS produced by rotenone, $\mathrm{CDNB}$ and possibly $\mathrm{MCB}$, can promote lysosome fission through actin clearance on lysosomes during PIKfyve inhibition, circumventing lysosome enlargement.

Our work suggest ROS species affect lysosome dynamics distinctly through different targets. Specifically, $\mathrm{H}_{2} \mathrm{O}_{2}$ stabilize microtubules and impaired lysosome mobility where such impairment maybe due to dysregulation of microtubule-motor function. Thus, $\mathrm{H}_{2} \mathrm{O}_{2}$ may prevent lysosome coalescence during acute PIKfyve inhibition and accelerate lysosome fragmentation during PIKfyve re-activation by disrupting lysosome motility and fusogenecity. Alternatively, 
rotenone, $\mathrm{CDNB}$, and $\mathrm{MCB}$, sources of superoxide production, promoted actin clearance from lysosomes, thereby relieving actin mass from lysosomes formed during PIKfyve inhibition that otherwise hinder fission, as suggested by Bissig et al (Bissig et al., 2019). Thus, these three ROS agonists through actin clearance seem to stimulate lysosome fission to counteract coalescence during acute PIKfyve inhibition and promote lysosome fragmentation during PIKfyve reactivation. Future work needs to address the specific targets of different ROS that affect lysosome dynamics distinctly. Additionally, lysosome enlargement from $\operatorname{PtdIns}(3,5) \mathrm{P}_{2}$ depletion accumulate intra-lysosomal proteins and lipids with pathophysiological consequences (Mironova et al., 2016; Zou et al., 2015). It would be highly informative if questions could be addressed regarding whether ROS can promote lysosome fission and membrane recycling to rescue enlarged lysosomes and promote redistribution of contents abnormally stored within enlarged lysosomes.

\subsubsection{Future directions}

Since our work demonstrated that different ROS and/or sites of ROS synthesis affect lysosome dynamics through different mechanisms, future work should address the exact targets of these distinct ROS and whether those targets are conserved. Since $\mathrm{H}_{2} \mathrm{O}_{2}$ impaired lysosome mobility, it would be ideal to observe if forced anchorage of microtubule motors on lysosomes rescue mobility as it was done with mitochondria through targeted kinesin-1 localization using FKBP-FRB rapamycin heterodimerization, where FKBP-LAMP1 can anchor FRB-Kif5B (kinesin-1) to lysosomes through rapamycin. Since $\mathrm{H}_{2} \mathrm{O}_{2}$ impaired lysosome mobility, whether such impairment prevents endolysosome and/or phagolysosome formation and cargo delivery to lysosomes are possibilities to address. Experiments could be designed to observe whether dextran trafficking to lysosomes are impaired following endocytosis, and/or whether phagosome 
maturation and cargo degradation is impeded upon $\mathrm{H}_{2} \mathrm{O}_{2}$ application, due to arrested late endosome-lysosome fusion and/or phagosome-lysosome fusion. We observed that $\mathrm{H}_{2} \mathrm{O}_{2}$ recruited clathrin and dynamin onto lysosomes, though this was not sufficient to alter lysosome dynamics on their own, possibly because of the need to recruit and dissociate; conceivably, $\mathrm{H} 2 \mathrm{O} 2$ may freeze clathrin and dynamin in place preventing dynamic assembly and disassembly needed for fission. Nevertheless, we did observe increased PtdIns(4)P levels upon $\mathrm{H}_{2} \mathrm{O}_{2}$ application (data not shown). Since PtdIns(4)P recruit clathrin and dynamin, $\mathrm{H}_{2} \mathrm{O}_{2}$ may increase lysosome associated PtdIns(4)P to recruit fission machinery components such as spastizin, spatacsin and AP-5 in addition to clathrin and dynamin to synergistically promote fission where clathrin and/or dynamin may be dispensable since membrane scission could be completed by other components.

Rotenone, CDNB and MCB, through superoxide affect lysosome dynamics distinctly from $\mathrm{H}_{2} \mathrm{O}_{2}$. Since superoxide production removed actin from lysosomes, it should be addressed whether actin turnover and/or polymerization is also affected at lysosome sites. If lysosome-associated actin turnover and/or polymerization is affected by superoxide, one could speculate whether this is a physiological function controlled by specific NOX enzymes since NOX2 is found on lysosomes ( $\mathrm{Li}$ et al., 2012). This could be tested by silencing NOX2 and looking at lysosome dynamics and actin-polymerization on lysosomes, including actin nucleation machinery such as WASH, cortactin and/or Arp-2/3 at lysosomes.

ROS generation may promote lysosome fission and membrane recycling as suggested from our work. It should be evaluated whether ROS agonists through superoxide generation can rescue lysosome enlargement from genetic models of $\operatorname{Ptd} \operatorname{Ins}(3,5) \mathrm{P}_{2}$ depletion and whether such rescue can redistribute proteins and lipids from enlarged lysosomes. 


\section{Section 6: Conclusion}




\subsection{Conclusion}

Lipid kinase PIKfyve through association and phosphorylation of D-5 hydroxyl of inositol head group of PtdIns(3)P, generate endolysosome and lysosome membrane lipid PtdIns(3,5) $\mathrm{P}_{2}$. PtdIns(3,5) $\mathrm{P}_{2}$ functions in autophagosome, phagosome and endosome maturation with lysosomes for formation of autolysosomes, phagolysosomes and endolysosomes respectively. Additionally, PtdIns(3,5) $\mathrm{P}_{2}$ regulate lysosome membrane and lumen recycling, lysosome associated ion channel activity, actin turnover, implicating role $\operatorname{Ptd} \operatorname{Ins}(3,5) \mathrm{P}_{2}$ as a critical signaling lipid from lysosome membrane. However, the most dramatic phenotype from $\operatorname{Ptd} \operatorname{Ins}(3,5) \mathrm{P}_{2}$ depletion is lysosome enlargement (Dayam et al., 2015; Hasegawa et al., 2016; Shisheva, 2008; Zou et al., 2015). Lysosomes undergo repeated fusion-fission cycles and/or transient kiss-and-run to maintain lysosome number and size (Saffi and Botelho, 2019). Our work demonstrate role of PtdIns $(3,5) \mathrm{P}_{2}$ in regulating lysosome fission, and depletion of $\operatorname{Ptd} \operatorname{Ins}(3,5) \mathrm{P}_{2}$ impair fission during continuous fusion to coalesce lysosomes for enlargement. Such coalescence is dependent on microtubules and associated motor protein complexes, dynein and kinesin-1. Additionally, nuclear translocation of TFEB transcription factor and lysosome gene expression during PIKfyve inhibition does not contribute to lysosome enlargement upon conditions of acute PIKfyve arrest. Thus, PtdIns(3,5) $\mathrm{P}_{2}$ regulate lysosome number and size, and depletion through acute PIKfyve inhibition enlarge lysosomes through coalescence without regulatory input from TFEB. Future work could characterize to address the specific target of $\operatorname{PtdIns}(3,5) \mathrm{P}_{2}$, such as WIPI proteins and/or actin nucleation machinery as discussed, that orchestrate lysosome fission.

Upon attempting to capture the dynamics of lysosome enlargement from apilimod through conventional fluorescence spinning disc confocal microscopy, we accidentally stumbled upon microscopy induced ROS stimulation to arrest apilimod induced lysosome enlargement. Further 
observations suggested role of ROS in regulating lysosome fusion and fission during acute PIKfyve inhibition. However, depending on the ROS species and/or site of ROS synthesis, lysosomes dynamics were affected distinctly. $\mathrm{H}_{2} \mathrm{O}_{2}$ impaired lysosome mobility, not through enhanced microtubule structure but maybe through impeded motor activity, to arrest lysosome coalescence from PIKfyve inhibition. However, superoxide generation from mitochondrial ETC complex I, thioredoxin reductase, or glutathione inhibition, through rotenone, $\mathrm{CDNB}$, or $\mathrm{MCB}$ respectively, depolymerised microtubules without affecting lysosome mobility. Instead, ROS generation from such manipulations promoted actin clearance from lysosomes, and actin depolymerisation inhibited lysosome coalescence during PIKfyve inhibition and enhanced lysosome fragmentation during PIKfyve re-activation, suggesting superoxide promote lysosome associated actin clearance to stimulate fission. Future work could address specific targets of ROS species that could regulate lysosome fusion and fission as discussed, such as microtubule motors and actin nucleation machinery. 


\section{Appendices:}
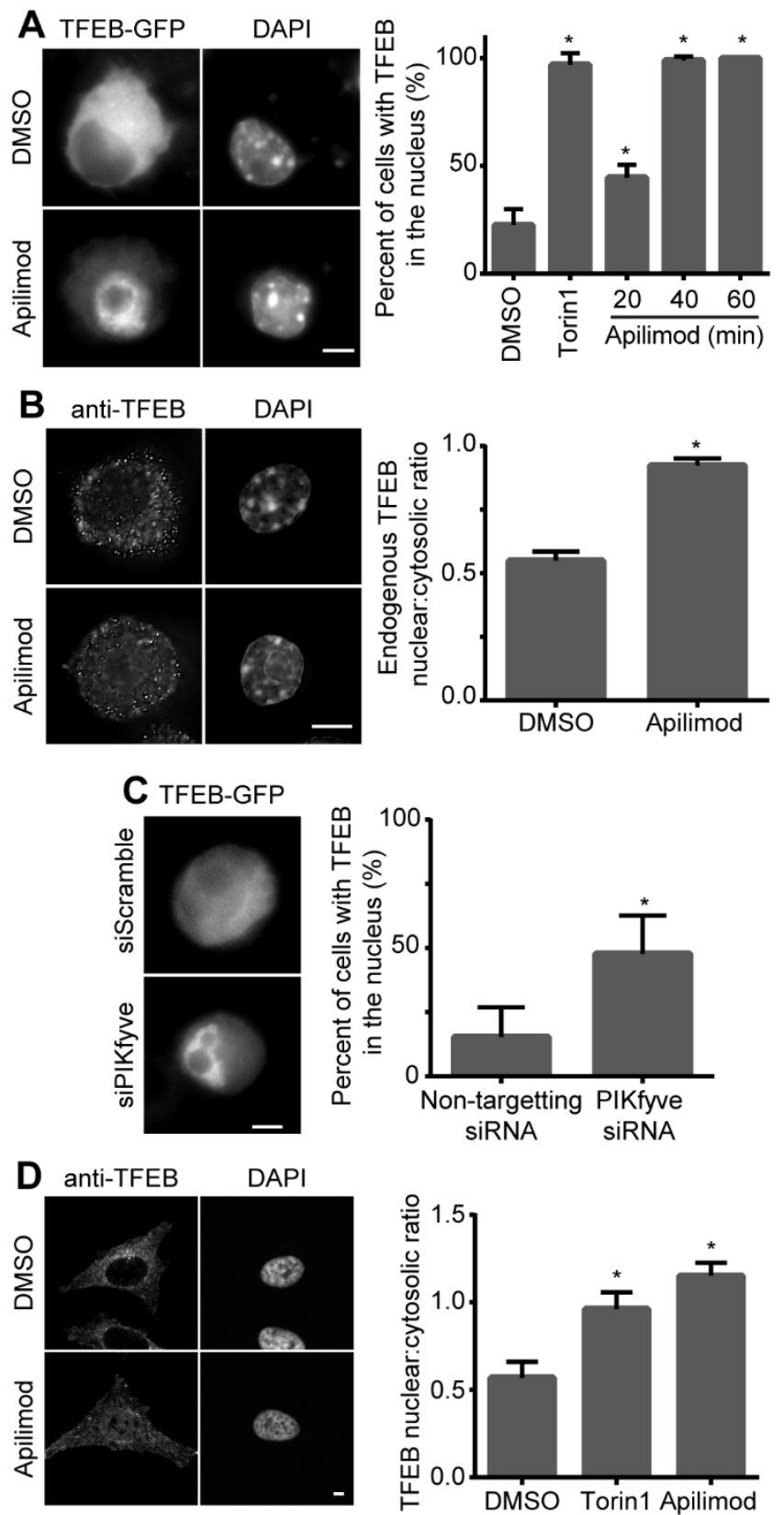

Supplemental Figure A1: PIKfyve inhibition cause nuclear translocation of TFEB. (A, B)

RAW cells expressing transfected TFEB-GFP (A), or immunostained for endogenous TFEB

(B), post vehicle or $20 \mathrm{nM}$ apilimod $1 \mathrm{~h}$ treatment. (C) RAW cells silenced for PIKfyve and 
transfected with TFEB-GFP. (D) HeLa cells treated with vehicle or $20 \mathrm{nM}$ apilimod $1 \mathrm{~h}$ followed by immunostaining against endogenous TFEB. (A-D) Nuclei stained with DAPI. Nuclear translocation of TFEB scored as percentage of cells with TFEB localized to DAPI positive nucleus $(\mathbf{A}, \mathbf{C})$, or quantifying nuclear to cytosol endogenous TFEB fluorescence intensity ratio (B, D). For all experiments, data represent \pm SEM from 3 independent experiments with 50-200 cells counted per condition per experiment. Scale bars: $5 \mu \mathrm{M}$.

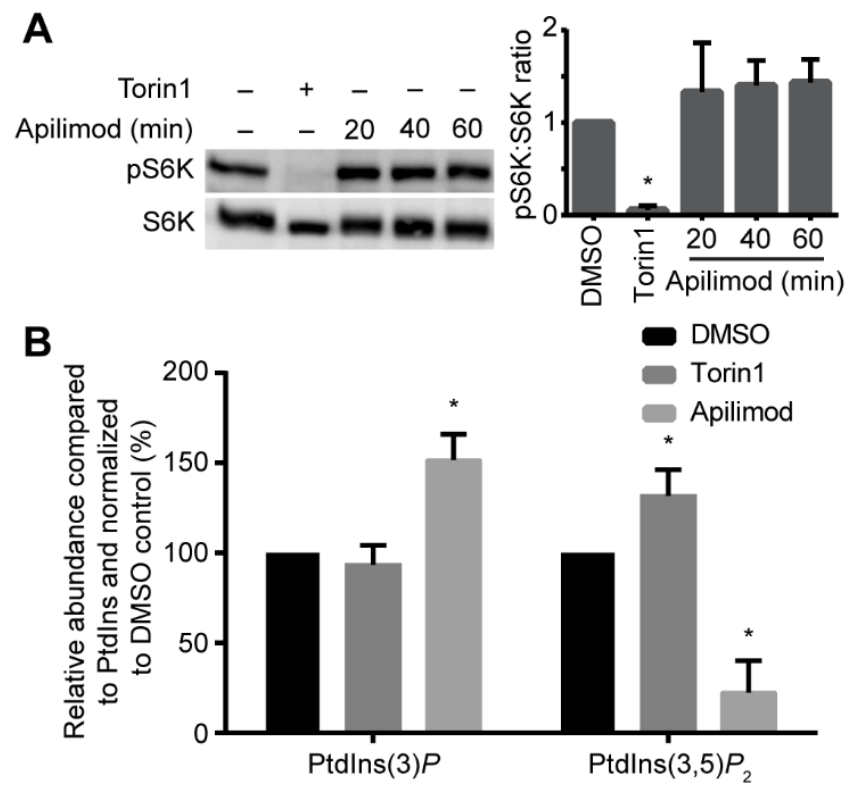


Supplemental Figure B1: PIKfyve and mTOR function independently. (A) RAW cells treated with Torin1 to inhibit mTOR or apilimod at indicated times followed by western blot against phosphorylated $\mathrm{Thr}^{389}$-p70S6K and total p70S6K. Ratio of $\mathrm{Thr}^{389}{ }^{\mathrm{p}} \mathrm{p} 70 \mathrm{~S} 6 \mathrm{~K}$ to total p70S6K quantified. Data represent \pm s.d. from at least 3 independent experiments. (B) RAW cells treated with Torin 1 to inhibit mTOR or apilimod $20 \mathrm{nM} 60 \mathrm{~min}$ followed by incorporation of ${ }^{3} \mathrm{H}-m y o$-inositol and HPLC coupled flow scintillation to detect PtdIns(3)P and PtdIns $(3,5) \mathrm{P}_{2}$ levels. Data represent $\underline{ \pm}$ s.d. from at least 3 independent experiments.
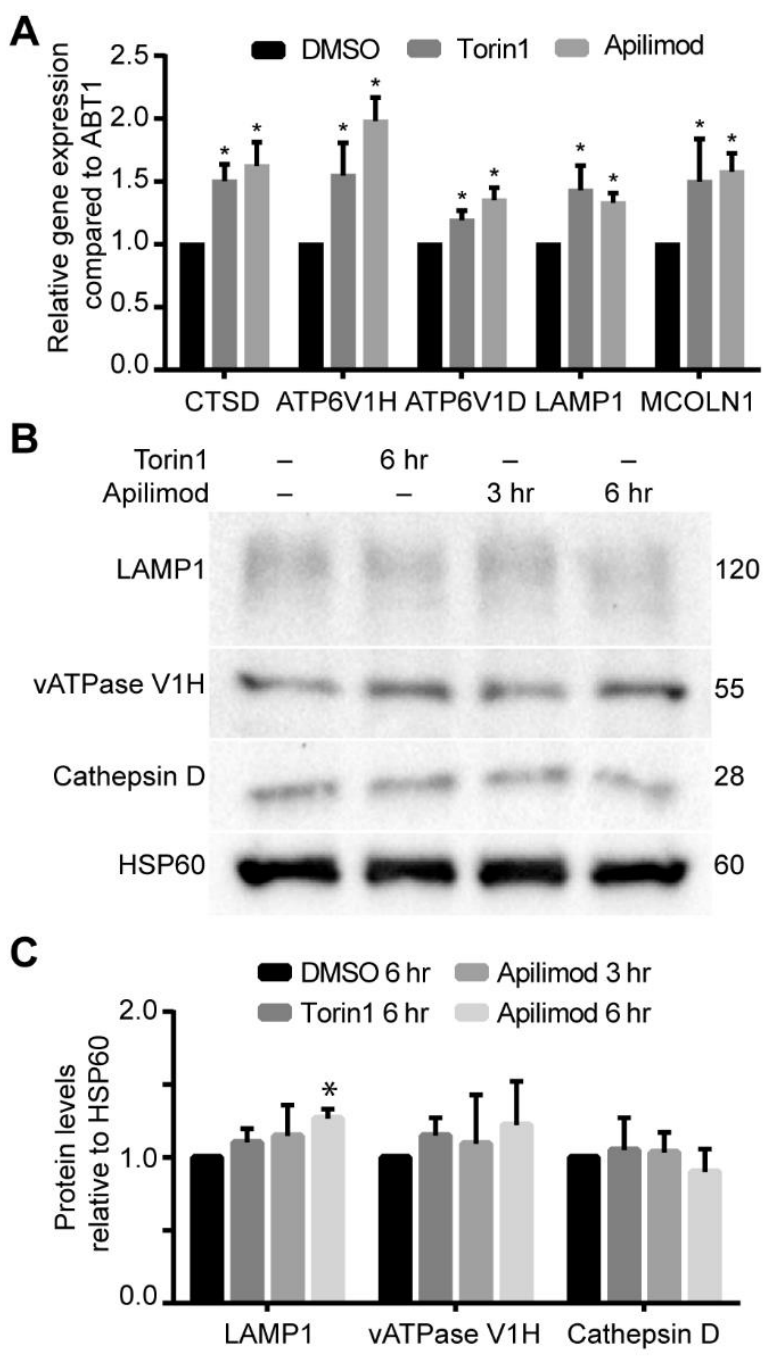
Supplemental Figure C1: Acute PIKfyve inhibition increase expression of lysosome genes without affecting protein expression. (A) RAW cells inhibited for mTOR with Torin1 and PIKfyve with apilimod, followed by qRT-PCR for select lysosome genes normalized to ABT1. Shown is \pm SEM from seven independent experiments. (B) RAW cells inhibited for mTOR with Torin1 and PIKfyve with apilimod, followed by western blot against select proteins. Numbers on right refer to molecular weight (kDA). (C) Quantification of proteins from (B) normalized to HSP60. Shown is \pm SEM from four independent experiments. 

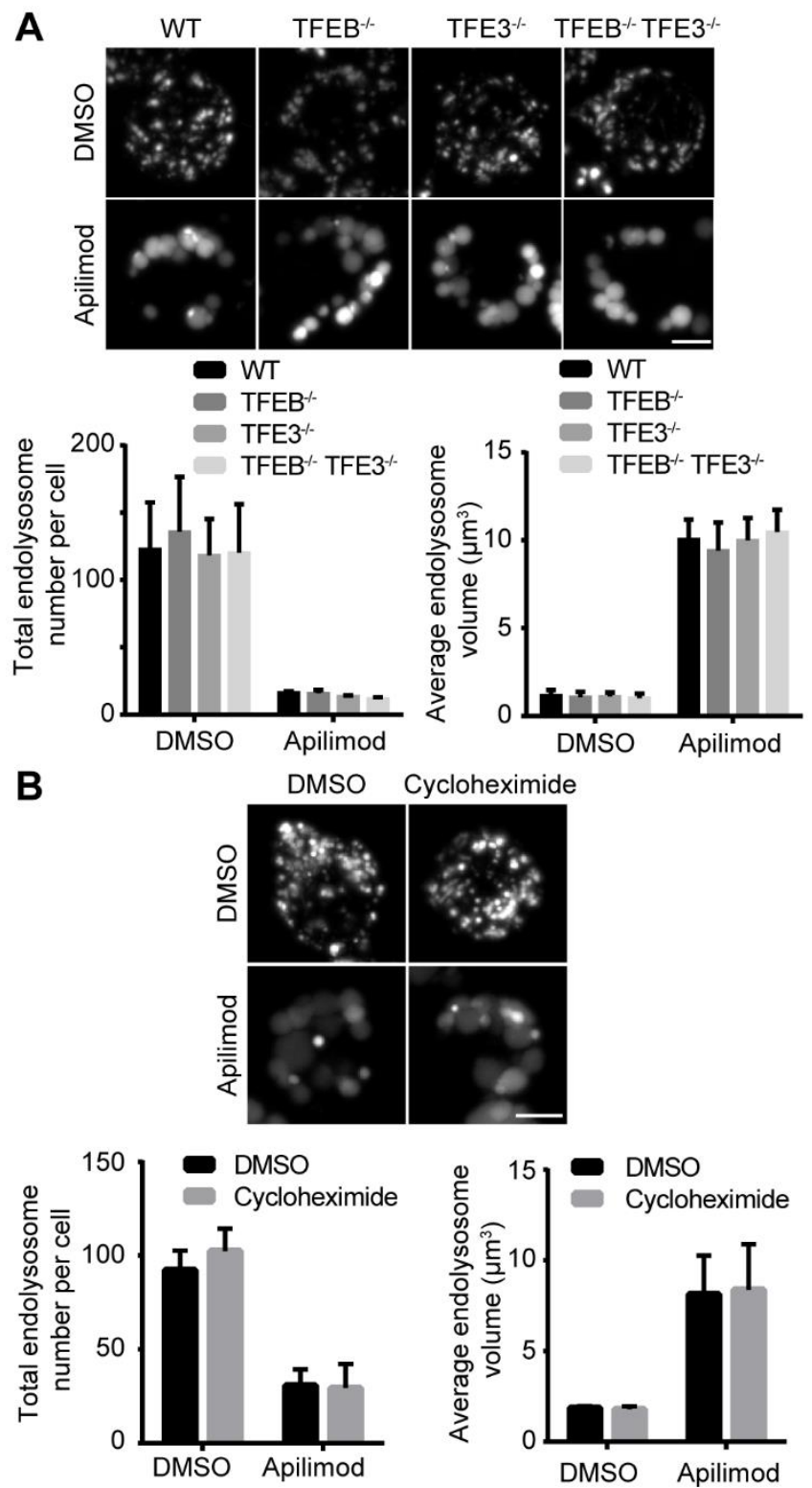


\section{Supplemental Figure D1: TFEB and protein synthesis do not contribute to lysosome}

enlargement. (A) RAW cells wild-type or depleted for TFEB and/or TFE3 pre-labelled for lysosomes with Lucifer yellow, followed by treatment with vehicle or apilimod $20 \mathrm{nM} 1 \mathrm{~h}$. Lysosome number per cell and average volume quantified. (B) RAW cells pre-labelled for lysosomes with Lucifer yellow, followed by vehicle or cycloheximide treatment to inhibit protein synthesis in absence or presence of apilimod. Lysosome number per cell and average volume quantified. In all cases, data represent \pm SEM from 3 independent experiments with 100 cells counted per condition. Scale bars: $5 \mu \mathrm{M}$. 


\section{References:}

Aguet, F., Antonescu, C. N., Mettlen, M., Schmid, S. L. and Danuser, G. (2013). Advances in analysis of low signal-to-noise images link dynamin and AP2 to the functions of an endocytic checkpoint. Developmental cell 26, 279-91.

Almeida-Souza, L., Frank, R. A. W., García-Nafría, J., Colussi, A., Gunawardana, N., Johnson, C. M., Yu, M., Howard, G., Andrews, B., Vallis, Y., et al. (2018). A Flat BAR Protein Promotes Actin Polymerization at the Base of Clathrin-Coated Pits. Cell 174, 325337.e14.

André-Lévigne, D., Modarressi, A., Pepper, M. and Pittet-Cuénod, B. (2017). Reactive Oxygen Species and NOX Enzymes Are Emerging as Key Players in Cutaneous Wound Repair. International Journal of Molecular Sciences 18, 2149.

Ba, Q., Raghavan, G., Kiselyov, K. and Yang, G. (2018). Whole-Cell Scale Dynamic Organization of Lysosomes Revealed by Spatial Statistical Analysis. Cell reports 23, 35913606.

Baker, R. W. and Hughson, F. M. (2016). Chaperoning SNARE assembly and disassembly. Nature reviews. Molecular cell biology 17, 465-79.

Ballabio, A. (2016). The awesome lysosome. EMBO molecular medicine 8, 73-6.

Bastiani, M. and Parton, R. G. (2010). Caveolae at a glance. Journal of cell science 123, 38316.

Bi, X. and Liao, G. (2010). Cholesterol in Niemann-Pick Type C disease. Sub-cellular biochemistry 51, 319-35.

Bibov, M. Y., Kuzmin, A. V., Alexandrova, A. A., Chistyakov, V. A., Dobaeva, N. M. and Kundupyan, O. L. (2018). Role of the reactive oxygen species induced DNA damage in human spermatozoa dysfunction. AME Medical Journal 3, 19-19.

Bissig, C. and Gruenberg, J. (2013). Lipid sorting and multivesicular endosome biogenesis. Cold Spring Harbor perspectives in biology 5, a016816.

Bissig, C., Hurbain, I., Raposo, G. and van Niel, G. (2017). PIKfyve activity regulates 
reformation of terminal storage lysosomes from endolysosomes. Traffic 18, 747-757.

Bissig, C., Croisé, P., Heiligenstein, X., Hurbain, I., Lenk, G. M., Kaufman, E., Sannerud, R., Annaert, W., Meisler, M. H., Weisman, L. S., et al. (2019). PIKfyve complex regulates early melanosome homeostasis required for physiological amyloid formation. Journal of Cell Science jcs.229500.

Blajeski, A. L., Phan, V. A., Kottke, T. J. and Kaufmann, S. H. (2002). G(1) and G(2) cellcycle arrest following microtubule depolymerization in human breast cancer cells. The Journal of clinical investigation 110, 91-9.

Blander, J. M. (2008). Phagocytosis and antigen presentation: a partnership initiated by Tolllike receptors. Annals of the Rheumatic Diseases 67, iii44-iii49.

Bonangelino, C. J., Nau, J. J., Duex, J. E., Brinkman, M., Wurmser, A. E., Gary, J. D., Emr, S. D. and Weisman, L. S. (2002). Osmotic stress-induced increase of phosphatidylinositol 3,5-bisphosphate requires Vac14p, an activator of the lipid kinase Fab1p. The Journal of cell biology 156, 1015-28.

Boutry, M., Branchu, J., Lustremant, C., Pujol, C., Pernelle, J., Matusiak, R., Seyer, A., Poirel, M., Chu-Van, E., Pierga, A., et al. (2018). Inhibition of Lysosome Membrane Recycling Causes Accumulation of Gangliosides that Contribute to Neurodegeneration. Cell Reports 23, 3813-3826.

Bright, N. A., Reaves, B. J., Mullock, B. M. and Luzio, J. P. (1997). Dense core lysosomes can fuse with late endosomes and are re-formed from the resultant hybrid organelles. Journal of cell science 110 ( Pt 17), 2027-40.

Bright, N. A., Gratian, M. J. and Luzio, J. P. (2005). Endocytic Delivery to Lysosomes Mediated by Concurrent Fusion and Kissing Events in Living Cells. Current Biology 15, $360-365$.

Bright, N. A., Davis, L. J. and Luzio, J. P. (2016). Endolysosomes Are the Principal Intracellular Sites of Acid Hydrolase Activity. Current Biology 26, 2233-2245.

Brown, D. I. and Griendling, K. K. (2009). Nox proteins in signal transduction. Free Radical Biology and Medicine 47, 1239-1253. 
Bucci, C., Thomsen, P., Nicoziani, P., McCarthy, J. and van Deurs, B. (2000). Rab7: A Key to Lysosome Biogenesis. Molecular Biology of the Cell 11, 467-480.

Burbank, K. S. and Mitchison, T. J. (2006). Microtubule dynamic instability. Current biology: CB 16, R516-7.

Burgess, S. A., Walker, M. L., Sakakibara, H., Knight, P. J. and Oiwa, K. (2003). Dynein structure and power stroke. Nature 421, 715-718.

Busse, R. A., Scacioc, A., Krick, R., Pérez-Lara, Á., Thumm, M. and Kühnel, K. (2015). Characterization of PROPPIN-Phosphoinositide Binding and Role of Loop 6CD in PROPPIN-Membrane Binding. Biophysical journal 108, 2223-34.

Cadet, J. and Wagner, J. R. (2013). DNA base damage by reactive oxygen species, oxidizing agents, and UV radiation. Cold Spring Harbor perspectives in biology 5,.

Cantalupo, G., Alifano, P., Roberti, V., Bruni, C. B. and Bucci, C. (2001). Rab-interacting lysosomal protein (RILP): the Rab7 effector required for transport to lysosomes. The EMBO journal 20, 683-93.

Cantu-Medellin, N. and Kelley, E. E. (2013). Xanthine oxidoreductase-catalyzed reactive species generation: A process in critical need of reevaluation. Redox Biology 1, 353-358.

Castellano-Muñoz, M., Peng, A. W., Salles, F. T. and Ricci, A. J. (2012). Swept field laser confocal microscopy for enhanced spatial and temporal resolution in live-cell imaging. Microscopy and microanalysis : the official journal of Microscopy Society of America, Microbeam Analysis Society, Microscopical Society of Canada 18, 753-60.

Chang-Ileto, B., Frere, S. G., Chan, R. B., Voronov, S. V., Roux, A. and Di Paolo, G. (2011). Synaptojanin 1-Mediated PI(4,5)P2 Hydrolysis Is Modulated by Membrane Curvature and Facilitates Membrane Fission. Developmental Cell 20, 206-218.

Chang, H. C., Newmyer, S. L., Hull, M. J., Ebersold, M., Schmid, S. L. and Mellman, I. (2002). Hsc70 is required for endocytosis and clathrin function in Drosophila. The Journal of cell biology 159, 477-87.

Chang, J., Lee, S. and Blackstone, C. (2014). Spastic paraplegia proteins spastizin and 
spatacsin mediate autophagic lysosome reformation. Journal of Clinical Investigation 124, $5249-5262$.

Chatterjee, S., Noack, H., Possel, H., Keilhoff, G. and Wolf, G. (1999). Glutathione levels in primary glial cultures: monochlorobimane provides evidence of cell type-specific distribution. Glia 27, 152-61.

Chen, Y. and Yu, L. (2017). Recent progress in autophagic lysosome reformation. Traffic 18, $358-361$.

Cheng, J. P. X. and Nichols, B. J. (2016). Caveolae: One Function or Many?

Chintaluri, K., Goulden, B. D., Clemenza, C., Saffi, G., Miraglia, E., Hammond, G. R. V. and Botelho, R. J. (2018). Correction: The PH domain from the Toxoplasma gondii PHcontaining protein-1 (TgPH1) serves as an ectopic reporter of phosphatidylinositol 3phosphate in mammalian cells. PLOS ONE 13, e0201800.

Choy, C. H., Han, B. K. and Botelho, R. J. (2017). Phosphoinositide Diversity, Distribution, and Effector Function: Stepping Out of the Box. BioEssays 39, 1700121.

Choy, C. H., Saffi, G., Gray, M. A., Wallace, C., Dayam, R. M., Ou, Z.-Y. A., Lenk, G., Puertollano, R., Watkins, S. C. and Botelho, R. J. (2018). Lysosome enlargement during inhibition of the lipid kinase PIKfyve proceeds through lysosome coalescence. Journal of Cell Science 131, jcs213587.

Clarke, M., Köhler, J., Heuser, J. and Gerisch, G. (2002). Endosome Fusion and MicrotubuleBased Dynamics in the Early Endocytic Pathway of Dictyostelium. Traffic 3, 791-800.

Cockburn, J. J. B., Hesketh, S. J., Mulhair, P., Thomsen, M., O'connell, M. J. and Correspondence, M. W. (2018). Insights into Kinesin-1 Activation from the Crystal Structure of KLC2 Bound to JIP3.

Currinn, H., Guscott, B., Balklava, Z., Rothnie, A. and Wassmer, T. (2016). APP controls the formation of $\mathrm{PI}(3,5) \mathrm{P} 2$ vesicles through its binding of the PIKfyve complex. Cellular and Molecular Life Sciences 73, 393-408.

Dalle-Donne, I., Giustarini, D., Rossi, R., Colombo, R. and Milzani, A. (2003). Reversible S- 
glutathionylation of Cys 374 regulates actin filament formation by inducing structural changes in the actin molecule. Free radical biology \& medicine 34, 23-32.

Dayam, R. M., Saric, A., Shilliday, R. E. and Botelho, R. J. (2015). The PhosphoinositideGated Lysosomal Ca2+ Channel, TRPML1, Is Required for Phagosome Maturation. Traffic 16, 1010-1026.

de Lartigue, J., Polson, H., Feldman, M., Shokat, K., Tooze, S. A., Urbé, S. and Clague, M. J. (2009). PIKfyve regulation of endosome-linked pathways. Traffic (Copenhagen, Denmark) 10, 883-93.

Debattisti, V., Gerencser, A. A., Saotome, M., Das, S. and Hajnóczky, G. (2017). ROS Control Mitochondrial Motility through p38 and the Motor Adaptor Miro/Trak. Cell reports 21, 1667-1680.

Di Paolo, G. and De Camilli, P. (2006). Phosphoinositides in cell regulation and membrane dynamics.

Diao, J., Liu, R., Rong, Y., Zhao, M., Zhang, J., Lai, Y., Zhou, Q., Wilz, L. M., Li, J., Vivona, S., et al. (2015). ATG14 promotes membrane tethering and fusion of autophagosomes to endolysosomes. Nature 520, 563-566.

Doherty, G. J. and McMahon, H. T. (2009). Mechanisms of Endocytosis. Annual Review of Biochemistry 78, 857-902.

Dong, X., Shen, D., Wang, X., Dawson, T., Li, X., Zhang, Q., Cheng, X., Zhang, Y., Weisman, L. S., Delling, M., et al. (2010). PI(3,5)P2 controls membrane trafficking by direct activation of mucolipin $\mathrm{Ca} 2+$ release channels in the endolysosome. Nature Communications 1, 1-11.

Dove, S. K., Piper, R. C., McEwen, R. K., Yu, J. W., King, M. C., Hughes, D. C., Thuring, J., Holmes, A. B., Cooke, F. T., Michell, R. H., et al. (2004). Svp1p defines a family of phosphatidylinositol 3,5-bisphosphate effectors. The EMBO Journal 23, 1922-1933.

Dröse, S., Brandt, U. and Wittig, I. (2014). Mitochondrial respiratory chain complexes as sources and targets of thiol-based redox-regulation $3 B$. Proteins and Proteomics 1844, $1344-1354$. 
Du, W., Su, Q. P., Chen, Y., Zhu, Y., Jiang, D., Rong, Y., Zhang, S., Zhang, Y., Ren, H., Zhang, C., et al. (2016). Kinesin 1 Drives Autolysosome Tubulation. Developmental Cell 37, 326-336.

Duex, J. E., Tang, F. and Weisman, L. S. (2006). The Vac14p-Fig4p complex acts independently of Vac7p and couples PI3,5P2 synthesis and turnover. The Journal of cell biology 172, 693-704.

Echeverri, C. J., Paschal, B. M., Vaughan, K. T. and Vallee, R. B. (1996). Molecular characterization of the $50-\mathrm{kD}$ subunit of dynactin reveals function for the complex in chromosome alignment and spindle organization during mitosis. The Journal of Cell Biology 132, 617-633.

Efe, J. A., Botelho, R. J. and Emr, S. D. (2007). Atg18 regulates organelle morphology and Fab1 kinase activity independent of its membrane recruitment by phosphatidylinositol 3,5bisphosphate. Molecular biology of the cell 18, 4232-44.

Elkin, S. R., Lakoduk, A. M. and Schmid, S. L. (2016a). Endocytic pathways and endosomal trafficking: a primer. Wiener medizinische Wochenschrift (1946) 166, 196-204.

Elkin, S. R., Oswald, N. W., Reed, D. K., Mettlen, M., MacMillan, J. B. and Schmid, S. L. (2016b). Ikarugamycin: A Natural Product Inhibitor of Clathrin-Mediated Endocytosis. Traffic 17, 1139-1149.

Elmquist, S., Libelius, R., Lawoko, G. and Tågerud, S. (1992). Dextrans as markers for endocytosis in innervated and denervated skeletal muscle. Muscle \& Nerve 15, 876-884.

Endow, S. A., Kull, F. J. and Liu, H. (2010). Kinesins at a glance. Journal of cell science 123, 3420-4.

Eskelinen, E.-L. and Saftig, P. (2009). Autophagy: A lysosomal degradation pathway with a central role in health and disease. Biochimica et Biophysica Acta (BBA) - Molecular Cell Research 1793, 664-673.

Flannagan, R. S., Jaumouillé, V. and Grinstein, S. (2012). The Cell Biology of Phagocytosis. Annual Review of Pathology: Mechanisms of Disease 7, 61-98. 
Forman, H. J., Zhang, H. and Rinna, A. (2009). Glutathione: overview of its protective roles, measurement, and biosynthesis. Molecular aspects of medicine 30, 1-12.

Forrester, S. J., Kikuchi, D. S., Hernandes, M. S., Xu, Q. and Griendling, K. K. (2018). Reactive Oxygen Species in Metabolic and Inflammatory Signaling. Circulation Research 122, 877-902.

Freeman, S. A. and Grinstein, S. (2018). Resolution of macropinosomes, phagosomes and autolysosomes: Osmotically driven shrinkage enables tubulation and vesiculation. Traffic 19, 965-974.

Gennerich, A. and Vale, R. D. (2009). Walking the walk: how kinesin and dynein coordinate their steps. Current opinion in cell biology 21, 59-67.

Gerber, A. J. and Peterson, B. S. (2008). What is an image? Journal of the American Academy of Child and Adolescent Psychiatry 47, 245-8.

Godi, A., Campli, A. Di, Konstantakopoulos, A., Tullio, G. Di, Alessi, D. R., Kular, G. S., Daniele, T., Marra, P., Lucocq, J. M. and Matteis, M. A. De (2004). FAPPs control Golgi-to-cell-surface membrane traffic by binding to ARF and PtdIns(4)P. Nature Cell Biology 6, 393-404.

Goldstein, J. L., Anderson, R. G. and Brown, M. S. (1982). Receptor-mediated endocytosis and the cellular uptake of low density lipoprotein. Ciba Foundation symposium 77-95.

Gomez, T. S., Gorman, J. A., de Narvajas, A. A.-M., Koenig, A. O. and Billadeau, D. D. (2012). Trafficking defects in WASH-knockout fibroblasts originate from collapsed endosomal and lysosomal networks. Molecular biology of the cell 23, 3215-28.

Gopaldass, N., Fauvet, B., Lashuel, H., Roux, A. and Mayer, A. (2017). Membrane scission driven by the PROPPIN Atg18. The EMBO Journal 36, 3274-3291.

Gordon, S. (2016). Phagocytosis: An Immunobiologic Process. Immunity 44, 463-475.

Groemping, Y. and Rittinger, K. (2005). Activation and assembly of the NADPH oxidase: a structural perspective. The Biochemical journal 386, 401-16.

Guardia, C. M., Farías, G. G., Jia, R., Pu, J. and Bonifacino, J. S. (2016). BORC Functions 
Upstream of Kinesins 1 and 3 to Coordinate Regional Movement of Lysosomes along Different Microtubule Tracks. Cell reports 17, 1950-1961.

Hamacher-Brady, A., Stein, H. A., Turschner, S., Toegel, I., Mora, R., Jennewein, N., Efferth, T., Eils, R. and Brady, N. R. (2011). Artesunate activates mitochondrial apoptosis in breast cancer cells via iron-catalyzed lysosomal reactive oxygen species production. The Journal of biological chemistry 286, 6587-601.

Han, J., Pluhackova, K. and Böckmann, R. A. (2017). The Multifaceted Role of SNARE Proteins in Membrane Fusion. Frontiers in physiology 8, 5.

Hanahan, D. and Weinberg, R. A. (2011). Hallmarks of cancer: the next generation. Cell 144, 646-74.

Harrison, R. E., Bucci, C., Vieira, O. V, Schroer, T. A. and Grinstein, S. (2003). Phagosomes fuse with late endosomes and/or lysosomes by extension of membrane protrusions along microtubules: role of Rab7 and RILP. Molecular and cellular biology 23, 6494-506.

Hasegawa, J., Iwamoto, R., Otomo, T., Nezu, A., Hamasaki, M. and Yoshimori, T. (2016). Autophagosome-lysosome fusion in neurons requires INPP5E, a protein associated with Joubert syndrome. The EMBO journal 35, 1853-67.

Heald, R. and Cohen-Fix, O. (2014). Morphology and function of membrane-bound organelles. Current opinion in cell biology 26, 79-86.

Heinz, S., Freyberger, A., Lawrenz, B., Schladt, L., Schmuck, G. and Ellinger-Ziegelbauer, H. (2017). Mechanistic Investigations of the Mitochondrial Complex I Inhibitor Rotenone in the Context of Pharmacological and Safety Evaluation. Scientific Reports 7, 45465.

Heuser, J. (1989). Changes in lysosome shape and distribution correlated with changes in cytoplasmic pH. Journal of Cell Biology 108, 855-864.

Hipolito, V. E. B., Ospina-Escobar, E. and Botelho, R. J. (2018). Lysosome remodelling and adaptation during phagocyte activation. Cellular Microbiology 20, e12824.

Hirst, J., Borner, G. H. H., Edgar, J., Hein, M. Y., Mann, M., Buchholz, F., Antrobus, R. and Robinson, M. S. (2013). Interaction between AP-5 and the hereditary spastic 
paraplegia proteins SPG11 and SPG15. Molecular biology of the cell 24, 2558-69.

Hirst, J., Itzhak, D. N., Antrobus, R., Borner, G. H. H. and Robinson, M. S. (2018). Role of the AP-5 adaptor protein complex in late endosome-to-Golgi retrieval. PLoS biology 16, e2004411.

Ho, C. Y., Alghamdi, T. A. and Botelho, R. J. (2012). Phosphatidylinositol-3,5-Bisphosphate: No Longer the Poor PIP2. Traffic 13, 1-8.

Ho, C. Y., Choy, C. H. and Botelho, R. J. (2016). Radiolabeling and Quantification of Cellular Levels of Phosphoinositides by High Performance Liquid Chromatography-coupled Flow Scintillation. Journal of Visualized Experiments e53529.

Hofmann, I. and Munro, S. (2006). An N-terminally acetylated Arf-like GTPase is localised to lysosomes and affects their motility. Journal of Cell Science 119, 1494-1503.

Hong, N. H., Qi, A. and Weaver, A. M. (2015). PI(3,5)P2 controls endosomal branched actin dynamics by regulating cortactin-actin interactions. The Journal of Cell Biology 210, 753769.

Huber, L. A. and Teis, D. (2016). Lysosomal signaling in control of degradation pathways. Current opinion in cell biology 39, 8-14.

Hung, C. H.-L., Cheng, S. S.-Y., Cheung, Y.-T., Wuwongse, S., Zhang, N. Q., Ho, Y.-S., Lee, S. M.-Y. and Chang, R. C.-C. (2018). A reciprocal relationship between reactive oxygen species and mitochondrial dynamics in neurodegeneration. Redox biology 14, 7-19.

Huotari, J. and Helenius, A. (2011). Endosome maturation. EMBO Journal 30, 3481-3500.

Hurley, J. H. (2010). The ESCRT complexes. Critical reviews in biochemistry and molecular biology 45, 463-87.

Icha, J., Weber, M., Waters, J. C. and Norden, C. (2017). Phototoxicity in live fluorescence microscopy, and how to avoid it. BioEssays 39, 1700003.

Ichikawa, M., Nishino, T., Nishino, T. and Ichikawa, A. (1992). Subcellular localization of xanthine oxidase in rat hepatocytes: high-resolution immunoelectron microscopic study combined with biochemical analysis. Journal of Histochemistry \& Cytochemistry 40, 1097- 
1103.

Ighodaro, O. M. and Akinloye, O. A. (2018). First line defence antioxidants-superoxide dismutase (SOD), catalase (CAT) and glutathione peroxidase (GPX): Their fundamental role in the entire antioxidant defence grid. Alexandria Journal of Medicine 54, 287-293.

Ikonomov, O. C., Sbrissa, D., Dondapati, R. and Shisheva, A. (2007). ArPIKfyve-PIKfyve interaction and role in insulin-regulated GLUT4 translocation and glucose transport in 3T3L1 adipocytes. Experimental Cell Research 313, 2404-2416.

Ikonomov, O. C., Sbrissa, D., Delvecchio, K., Xie, Y., Jin, J.-P., Rappolee, D. and Shisheva, A. (2011). The Phosphoinositide Kinase PIKfyve Is Vital in Early Embryonic Development. Journal of Biological Chemistry 286, 13404-13413.

Ikonomov, O. C., Sbrissa, D., Delvecchio, K., Rillema, J. A. and Shisheva, A. (2016). Unexpected severe consequences of Pikfyve deletion by aP2- or Aq-promoter-driven Cre expression for glucose homeostasis and mammary gland development. Physiological Reports 4,.

Inpanathan, S. and Botelho, R. J. (2019). The Lysosome Signaling Platform: Adapting With the Times. Frontiers in Cell and Developmental Biology 7, 113.

Jackson, L. P., Kelly, B. T., McCoy, A. J., Gaffry, T., James, L. C., Collins, B. M., Höning, S., Evans, P. R. and Owen, D. J. (2010). A large-scale conformational change couples membrane recruitment to cargo binding in the AP2 clathrin adaptor complex. Cell 141, 1220-9.

Jahreiss, L., Menzies, F. M. and Rubinsztein, D. C. (2008). The itinerary of autophagosomes: from peripheral formation to kiss-and-run fusion with lysosomes. Traffic (Copenhagen, Denmark) 9, 574-87.

Ježek, J., Cooper, K. F. and Strich, R. (2018). Reactive Oxygen Species and Mitochondrial Dynamics: The Yin and Yang of Mitochondrial Dysfunction and Cancer Progression. Antioxidants (Basel, Switzerland) 7,.

Jiang, P., Nishimura, T., Sakamaki, Y., Itakura, E., Hatta, T., Natsume, T. and Mizushima, N. (2014). The HOPS complex mediates autophagosome-lysosome fusion through 
interaction with syntaxin 17. Molecular Biology of the Cell 25, 1327-1337.

Jin, N., Chow, C. Y., Liu, L., Zolov, S. N., Bronson, R., Davisson, M., Petersen, J. L., Zhang, Y., Park, S., Duex, J. E., et al. (2008). EMBO open VAC14 nucleates a protein complex essential for the acute interconversion of PI3P and $\mathrm{PI}(3,5) \mathrm{P} 2$ in yeast and mouse. The EMBO Journal 27, 3221-3234.

Jin, N., Jin, Y. and Weisman, L. S. (2017). Early protection to stress mediated by CDKdependent PI3,5P2 signaling from the vacuole/lysosome. The Journal of cell biology $\mathbf{2 1 6}$, 2075-2090.

Johansen, T. and Lamark, T. (2011). Selective autophagy mediated by autophagic adapter proteins. Autophagy 7, 279-296.

Jun, Y. W., Wang, T., Hwang, S., Kim, D., Ma, D., Kim, K. H., Kim, S., Jung, J. and Ahn, K. H. (2018). A Ratiometric Two-Photon Fluorescent Probe for Tracking Lysosomal ATP: Direct In Cellulo Observation of Lysosomal Membrane Fusion Processes. Angewandte Chemie International Edition 57, 10142-10147.

Kamencic, H., Lyon, A., Paterson, P. G. and Juurlink, B. H. J. (2000). Monochlorobimane Fluorometric Method to Measure Tissue Glutathione. Analytical Biochemistry 286, 35-37.

Kanagaraj, P., Gautier-Stein, A., Riedel, D., Schomburg, C., Cerdà, J., Vollack, N. and Dosch, R. (2014). Souffle/Spastizin Controls Secretory Vesicle Maturation during Zebrafish Oogenesis. PLoS Genetics 10, e1004449.

Kato, Y., Miyakawa, T. and Tanokura, M. (2018). Overview of the mechanism of cytoskeletal motors based on structure. Biophysical reviews 10, 571-581.

Khaminets, A., Behl, C. and Dikic, I. (2016). Ubiquitin-Dependent And Independent Signals In Selective Autophagy. Trends in cell biology 26, 6-16.

Khundadze, M., Kollmann, K., Koch, N., Biskup, C., Nietzsche, S., Zimmer, G., Hennings, J. C., Huebner, A. K., Symmank, J., Jahic, A., et al. (2013). A Hereditary Spastic Paraplegia Mouse Model Supports a Role of ZFYVE26/SPASTIZIN for the Endolysosomal System. PLoS Genetics 9, e1003988. 
Kikkawa, M. (2013). Big steps toward understanding dynein. The Journal of cell biology 202, $15-23$.

Kim, G., Weiss, S. J. and Levine, R. L. (2014a). Methionine oxidation and reduction in proteins. Biochimica et biophysica acta 1840, 901-5.

Kim, G. H. E., Dayam, R. M., Prashar, A., Terebiznik, M. and Botelho, R. J. (2014b). PIKfyve Inhibition Interferes with Phagosome and Endosome Maturation in Macrophages. Traffic 15, 1143-1163.

King, S. M. (2016). Axonemal Dynein Arms. Cold Spring Harbor perspectives in biology 8,.

Klionsky, D. J., Eskelinen, E. L. and Deretic, V. (2014). Autophagosomes, phagosomes, autolysosomes, phagolysosomes, autophagolysosomes... Wait, I'm confused. Autophagy 10, $549-551$.

Klumperman, J. and Raposo, G. (2014). The complex ultrastructure of the endolysosomal system. Cold Spring Harbor Perspectives in Biology 6, a016857.

Krajcovic, M., Krishna, S., Akkari, L., Joyce, J. A. and Overholtzer, M. (2013). mTOR regulates phagosome and entotic vacuole fission. Molecular Biology of the Cell 24, 37363745.

Krishna, S., Palm, W., Lee, Y., Yang, W., Bandyopadhyay, U., Xu, H., Florey, O., Thompson, C. B. and Overholtzer, M. (2016). PIKfyve Regulates Vacuole Maturation and Nutrient Recovery following Engulfment. Developmental Cell 38, 536-547.

Landino, L. M., Moynihan, K. L., Todd, J. V and Kennett, K. L. (2004). Modulation of the redox state of tubulin by the glutathione/glutaredoxin reductase system q. Biochemical and Biophysical Research Communications 314, 555-560.

Lawe, D. C., Chawla, A., Merithew, E., Dumas, J., Carrington, W., Fogarty, K., Lifshitz, L., Tuft, R., Lambright, D. and Corvera, S. (2002). Sequential Roles for Phosphatidylinositol 3-Phosphate and Rab5 in Tethering and Fusion of Early Endosomes via Their Interaction with EEA1. Journal of Biological Chemistry 277, 8611-8617.

Lee, S. R., Kwon, K. S., Kim, S. R. and Rhee, S. G. (1998). Reversible inactivation of protein- 
tyrosine phosphatase 1B in A431 cells stimulated with epidermal growth factor. The Journal of biological chemistry 273, 15366-72.

LEE, C.-F., LIU, C.-Y., HSIEH, R.-H. and WEI, Y.-H. (2005). Oxidative Stress-Induced Depolymerization of Microtubules and Alteration of Mitochondrial Mass in Human Cells. Annals of the New York Academy of Sciences 1042, 246-254.

Lemarie, A. and Grimm, S. (2011). Mitochondrial respiratory chain complexes: apoptosis sensors mutated in cancer? Oncogene 30, 3985-4003.

Lesnefsky, E. J., Chen, Q., Tandler, B. and Hoppel, C. L. (2017). Mitochondrial Dysfunction and Myocardial Ischemia-Reperfusion: Implications for Novel Therapies. Annual Review of Pharmacology and Toxicology 57, 535-565.

Li, N., Li, B., Brun, T., Deffert-Delbouille, C., Mahiout, Z., Daali, Y., Ma, X.-J., Krause, K.H. and Maechler, P. (2012). NADPH Oxidase NOX2 Defines a New Antagonistic Role for Reactive Oxygen Species and cAMP/PKA in the Regulation of Insulin Secretion. Diabetes 61, 2842-2850.

Lubos, E., Loscalzo, J. and Handy, D. E. (2011). Glutathione peroxidase-1 in health and disease: from molecular mechanisms to therapeutic opportunities. Antioxidants \& redox signaling 15, 1957-97.

Luzio, J. P., Pryor, P. R. and Bright, N. A. (2007). Lysosomes: fusion and function. Nature Reviews Molecular Cell Biology 8, 622-632.

Luzio, J. P., Hackmann, Y., Dieckmann, N. M. G. and Griffiths, G. M. (2014). The biogenesis of lysosomes and lysosome-related organelles. Cold Spring Harbor perspectives in biology 6, a016840.

Mackeh, R., Lorin, S., Ratier, A., Mejdoubi-Charef, N., Baillet, A., Bruneel, A., Hamaï, A., Codogno, P., Poüs, C. and Perdiz, D. (2014). Reactive oxygen species, AMP-activated protein kinase, and the transcription cofactor p300 regulate $\alpha$-tubulin acetyltransferase- 1 ( $\alpha$ TAT-1/MEC-17)-dependent microtubule hyperacetylation during cell stress. The Journal of biological chemistry 289, 11816-28.

Magder, S. (2006). Reactive oxygen species: toxic molecules or spark of life? Critical Care 10, 
208.

Marengo, B., Nitti, M., Furfaro, A. L., Colla, R., Ciucis, C. De, Marinari, U. M., Pronzato, M. A., Traverso, N. and Domenicotti, C. (2016). Redox Homeostasis and Cellular Antioxidant Systems: Crucial Players in Cancer Growth and Therapy. Oxidative medicine and cellular longevity 2016, 6235641.

Marks, M. S., Heijnen, H. F. G. and Raposo, G. (2013). Lysosome-related organelles: Unusual compartments become mainstream. Current Opinion in Cell Biology 25, 495-505.

Mauvezin, C., Neisch, A. L., Ayala, C. I., Kim, J., Beltrame, A., Braden, C. R., Gardner, M. K., Hays, T. S. and Neufeld, T. P. (2016). Coordination of autophagosome-lysosome fusion and transport by a Klp98A-Rab14 complex in Drosophila. Journal of cell science 129, 971-82.

McCluskey, A., Daniel, J. A., Hadzic, G., Chau, N., Clayton, E. L., Mariana, A., Whiting, A., Gorgani, N. N., Lloyd, J., Quan, A., et al. (2013). Building a Better Dynasore: The Dyngo Compounds Potently Inhibit Dynamin and Endocytosis. Traffic 14, 1272-1289.

Mccords, J. M. and Fridovich, I. (1969). Superoxide Dismutase AN ENZYMIC FUNCTION FOR ERYTHROCUPREIN (HEMOCUPREIN)*.

Metzler, K. D., Fuchs, T. A., Nauseef, W. M., Reumaux, D., Roesler, J., Schulze, I., Wahn, V., Papayannopoulos, V. and Zychlinsky, A. (2011). Myeloperoxidase is required for neutrophil extracellular trap formation: implications for innate immunity. Blood 117, 953-9.

Mijaljica, D., Nazarko, T. Y., Brumell, J. H., Huang, W.-P., Komatsu, M., Prescott, M., Simonsen, A., Yamamoto, A., Zhang, H., Klionsky, D. J., et al. (2012). Receptor protein complexes are in control of autophagy. Autophagy 8, 1701-1705.

Min, S. H., Suzuki, A., Stalker, T. J., Zhao, L., Wang, Y., McKennan, C., Riese, M. J., Guzman, J. F., Zhang, S., Lian, L., et al. (2014). Loss of PIKfyve in platelets causes a lysosomal disease leading to inflammation and thrombosis in mice. Nature communications 5, 4691.

Mindell, J. A. (2012). Lysosomal Acidification Mechanisms. Annual Review of Physiology 74, $69-86$. 
Mironova, Y. A., Lenk, G. M., Lin, J.-P., Lee, S. J., Twiss, J. L., Vaccari, I., Bolino, A., Havton, L. A., Min, S. H., Abrams, C. S., et al. (2016). PI(3,5)P2 biosynthesis regulates oligodendrocyte differentiation by intrinsic and extrinsic mechanisms. eLife $\mathbf{5}$,

Mittal, M., Siddiqui, M. R., Tran, K., Reddy, S. P. and Malik, A. B. (2014). Reactive oxygen species in inflammation and tissue injury. Antioxidants \& redox signaling 20, 1126-67.

Mizuno-Yamasaki, E., Medkova, M., Coleman, J. and Novick, P. (2010).

Phosphatidylinositol 4-Phosphate Controls Both Membrane Recruitment and a Regulatory Switch of the Rab GEF Sec2p. Developmental Cell 18, 828-840.

Möller, K., Sigurbjornsdottir, S., Arnthorsson, A. O., Pogenberg, V., Dilshat, R., Fock, V., Brynjolfsdottir, S. H., Bindesboll, C., Bessadottir, M., Ogmundsdottir, H. M., et al. (2019). MITF has a central role in regulating starvation-induced autophagy in melanoma. Scientific reports $\mathbf{9}, 1055$.

Morfini, G. A., Bosco, D. A., Brown, H., Gatto, R., Kaminska, A., Song, Y., Molla, L., Baker, L., Marangoni, M. N., Berth, S., et al. (2013). Inhibition of Fast Axonal Transport by Pathogenic SOD1 Involves Activation of p38 MAP Kinase. PLoS ONE 8, e65235.

Mostafavi, H., Thiyagarajan, S., Stratton, B. S., Karatekin, E., Warner, J. M., Rothman, J. E. and O'shaughnessy, B. (2017). Entropic forces drive self-organization and membrane fusion by SNARE proteins. PNAS 114,.

Motley, A., Bright, N. A., Seaman, M. N. J. and Robinson, M. S. (2003). Clathrin-mediated endocytosis in AP-2-depleted cells. The Journal of cell biology 162, 909-18.

Mrakovic, A., Kay, J. G., Furuya, W., Brumell, J. H. and Botelho, R. J. (2012). Rab7 and Arl8 GTPases are Necessary for Lysosome Tubulation in Macrophages. Traffic 13, 16671679.

Nabi, I. R. and Le, P. U. (2003). Caveolae/raft-dependent endocytosis. The Journal of cell biology 161, 673-7.

Nakanishi, S., Catt, K. J. and Balla, T. (1995). A wortmannin-sensitive phosphatidylinositol 4kinase that regulates hormone-sensitive pools of inositolphospholipids. Proceedings of the National Academy of Sciences of the United States of America 92, 5317-21. 
Napolitano, G. and Ballabio, A. (2016). TFEB at a glance. Journal of cell science 129, 247581.

Noack, A., Gericke, B., von Köckritz-Blickwede, M., Menze, A., Noack, S., Gerhauser, I., Osten, F., Naim, H. Y. and Löscher, W. (2018). Mechanism of drug extrusion by brain endothelial cells via lysosomal drug trapping and disposal by neutrophils. Proceedings of the National Academy of Sciences of the United States of America 115, E9590-E9599.

Nordberg, J., Zhong, L., Holmgren, A. and Arnér, E. S. (1998). Mammalian thioredoxin reductase is irreversibly inhibited by dinitrohalobenzenes by alkylation of both the redox active selenocysteine and its neighboring cysteine residue. The Journal of biological chemistry 273, 10835-42.

Odobasic, D., Kitching, A. R. and Holdsworth, S. R. (2016). Neutrophil-Mediated Regulation of Innate and Adaptive Immunity: The Role of Myeloperoxidase. Journal of immunology research 2016, 2349817.

Okamoto, K. (2014). Organellophagy: eliminating cellular building blocks via selective autophagy. The Journal of cell biology 205, 435-45.

Padgaonkar, V. A., Leverenz, V. R., Bhat, A. V, Pelliccia, S. E. and Giblin, F. J. (2015). Thioredoxin reductase activity may be more important than GSH level in protecting human lens epithelial cells against UVA light. Photochemistry and photobiology 91, 387-96.

Page, E., Goings, G. E., Upshaw-Earley, J. and Hanck, D. A. (1994). Endocytosis and uptake of lucifer yellow by cultured atrial myocytes and isolated intact atria from adult rats. Regulation and subcellular localization. Circulation research 75, 335-46.

Park, H.-S., Han, J.-H., Jung, S.-H., Lee, D.-H., Heo, K.-S. and Myung, C.-S. (2018). Antiapoptotic effects of autophagy via ROS regulation in microtubule-targeted and PDGFstimulated vascular smooth muscle cells. The Korean journal of physiology \& pharmacology: official journal of the Korean Physiological Society and the Korean Society of Pharmacology 22, 349-360.

Pfister, K. K., Shah, P. R., Hummerich, H., Russ, A., Cotton, J., Annuar, A. A., King, S. M. and Fisher, E. M. C. (2006). Genetic Analysis of the Cytoplasmic Dynein Subunit 
Families. PLoS Genetics 2, e1.

Piper, R. C., Dikic, I. and Lukacs, G. L. (2014). Ubiquitin-Dependent Sorting in Endocytosis. Cold Spring Harbor Perspectives in Biology 6, a016808-a016808.

Pocasap, P., Weerapreeyakul, N. and Thumanu, K. (2018). Structures of isothiocyanates attributed to reactive oxygen species generation and microtubule depolymerization in HepG2 cells. Biomedicine \& Pharmacotherapy 101, 698-709.

Polson, H. E. J., de Lartigue, J., Rigden, D. J., Reedijk, M., Urbé, S., Clague, M. J. and Tooze, S. A. (2010). Mammalian Atg18 (WIPI2) localizes to omegasome-anchored phagophores and positively regulates LC3 lipidation. Autophagy 6, 506-522.

Pryor, P. R., Mullock, B. M., Bright, N. A., Lindsay, M. R., Gray, S. R., Richardson, S. C. W., Stewart, A., James, D. E., Piper, R. C. and Luzio, J. P. (2004). Combinatorial SNARE complexes with VAMP7 or VAMP8 define different late endocytic fusion events. EMBO reports $\mathbf{5}, 590-5$.

Pu, J., Guardia, C. M., Keren-Kaplan, T. and Bonifacino, J. S. (2016). Mechanisms and functions of lysosome positioning. Journal of cell science 129, 4329-4339.

Randall, T. S., Yip, Y. Y., Wallock-Richards, D. J., Pfisterer, K., Sanger, A., Ficek, W., Steiner, R. A., Beavil, A. J., Parsons, M. and Dodding, M. P. (2017). A small-molecule activator of kinesin-1 drives remodeling of the microtubule network. Proceedings of the National Academy of Sciences of the United States of America 114, 13738-13743.

Rasineni, K., Donohue, T. M., Thomes, P. G., Yang, L., Tuma, D. J., McNiven, M. A. and Casey, C. A. (2017). Ethanol-induced steatosis involves impairment of lipophagy, associated with reduced Dynamin2 activity. Hepatology Communications 1, 501-512.

Rice, S., Lin², A. W., Safer³, D., Hart, C. L., Naberk, N., Carragher, B. O., Cain², S. M., Pechatnikova\#, E., Wilson-Kubalek ${ }^{2}$, E. M., Whittaker², M., et al. (1999). A structural change in the kinesin motor protein that drives motility.

Ripoll, L., Heiligenstein, X., Hurbain, I., Domingues, L., Figon, F., Petersen, K. J., Dennis, M. K., Houdusse, A., Marks, M. S., Raposo, G., et al. (2018). Myosin VI and branched actin filaments mediate membrane constriction and fission of melanosomal tubule carriers. 
Journal of Cell Biology 217, 2709-2726.

Rohde, G., Wenzel, D. and Haucke, V. (2002). A phosphatidylinositol (4,5)-bisphosphate binding site within mu2-adaptin regulates clathrin-mediated endocytosis. The Journal of cell biology 158, 209-14.

Rong, Y., Liu, M., Ma, L., Du, W., Zhang, H., Tian, Y., Cao, Z., Li, Y., Ren, H., Zhang, C., et al. (2012). Clathrin and phosphatidylinositol-4,5-bisphosphate regulate autophagic lysosome reformation. Nature Cell Biology 14, 924-934.

Roostalu, J. and Surrey, T. (2017). Microtubule nucleation: beyond the template. Nature Reviews Molecular Cell Biology 18, 702-710.

Rosales, C. and Uribe-Querol, E. (2017). Phagocytosis: A Fundamental Process in Immunity. BioMed research international 2017, 9042851.

Saffi, G. T. and Botelho, R. J. (2019). Lysosome Fission: Planning for an Exit. Trends in Cell Biology.

Sainath, R. and Gallo, G. (2015). The dynein inhibitor Ciliobrevin D inhibits the bidirectional transport of organelles along sensory axons and impairs NGF-mediated regulation of growth cones and axon branches. Developmental neurobiology 75, 757-77.

Sakai, J., Li, J., Subramanian, K. K., Mondal, S., Bajrami, B., Hattori, H., Jia, Y., Dickinson, B. C., Zhong, J., Ye, K., et al. (2012). Article Reactive Oxygen SpeciesInduced Actin Glutathionylation Controls Actin Dynamics in Neutrophils. Immunity 37, $1037-1049$.

Sakuma, S., Abe, M., Kohda, T. and Fujimoto, Y. (2015). Hydrogen peroxide generated by xanthine/xanthine oxidase system represses the proliferation of colorectal cancer cell line Caco-2. Journal of clinical biochemistry and nutrition 56, 15-9.

Sakurai, C., Hashimoto, H., Nakanishi, H., Arai, S., Wada, Y., Sun-Wada, G.-H., Wada, I. and Hatsuzawa, K. (2012). SNAP-23 regulates phagosome formation and maturation in macrophages. Molecular biology of the cell 23, 4849-63.

Salvi, M., Battaglia, V., Maria Brunati, A., La Rocca, N., Tibaldi, E., Pietrangeli, P., 
Marcocci, L., Mondovı, B., Rossi, C. A., Toninello, A., et al. (2007). Catalase Takes Part in Rat Liver Mitochondria Oxidative Stress Defense * and the.

Sandvig, K., Kavaliauskiene, S. and Skotland, T. (2018). Clathrin-independent endocytosis: an increasing degree of complexity. Histochemistry and cell biology 150, 107-118.

Saric, A., Hipolito, V. E. B., Kay, J. G., Canton, J., Antonescu, C. N. and Botelho, R. J. (2016). mTOR controls lysosome tubulation and antigen presentation in macrophages and dendritic cells. Molecular Biology of the Cell 27, 321-33.

Sasaki, T., Takasuga, S., Sasaki, J., Kofuji, S., Eguchi, S., Yamazaki, M. and Suzuki, A. (2009). Mammalian phosphoinositide kinases and phosphatases. Progress in Lipid Research 48, 307-343.

Satori, C. P., Henderson, M. M., Krautkramer, E. A., Kostal, V., Distefano, M. D., Distefano, M. M. and Arriaga, E. A. (2013). Bioanalysis of eukaryotic organelles. Chemical reviews 113, 2733-811.

Sbrissa, D., Ikonomov, O. C., Fu, Z., Ijuin, T., Gruenberg, J., Takenawa, T. and Shisheva, A. (2007). Core Protein Machinery for Mammalian Phosphatidylinositol 3,5-Bisphosphate Synthesis and Turnover That Regulates the Progression of Endosomal Transport. Journal of Biological Chemistry 282, 23878-23891.

Sbrissa, D., Naisan, G., Ikonomov, O. C. and Shisheva, A. (2018). Apilimod, a candidate anticancer therapeutic, arrests not only PtdIns(3,5)P2 but also PtdIns5P synthesis by PIKfyve and induces bafilomycin A1-reversible aberrant endomembrane dilation. PLOS ONE 13, e0204532.

Schieber, M. and Chandel, N. S. (2014). ROS function in redox signaling and oxidative stress. Current biology : CB 24, R453-62.

Schmid, S. L. (2017). Reciprocal regulation of signaling and endocytosis: Implications for the evolving cancer cell. The Journal of cell biology 216, 2623-2632.

Schrader, M. and Fahimi, H. D. (2008). The peroxisome: still a mysterious organelle. Histochemistry and cell biology 129, 421-40. 
Scott, A., Gaspar, J., Stuchell-Brereton, M. D., Alam, S. L., Skalicky, J. J. and Sundquist, W. I. (2005). Structure and ESCRT-III protein interactions of the MIT domain of human VPS4A. Proceedings of the National Academy of Sciences 102, 13813-13818.

Settembre, C., Di Malta, C., Polito, V. A., Arencibia, M. G., Vetrini, F., Erdin, S., Erdin, S. U., Huynh, T., Medina, D., Colella, P., et al. (2011). TFEB Links Autophagy to Lysosomal Biogenesis. Science 332, 1429-1433.

Sharma, L. K., Lu, J. and Bai, Y. (2009). Mitochondrial respiratory complex I: structure, function and implication in human diseases. Current medicinal chemistry 16, 1266-77.

Sharma, P., Jha, A. B., Dubey, R. S. and Pessarakli, M. (2012). Reactive Oxygen Species, Oxidative Damage, and Antioxidative Defense Mechanism in Plants under Stressful Conditions. Journal of Botany 2012, 1-26.

Sharma, G., Guardia, C. M., Roy, A., Vassilev, A., Saric, A., Griner, L. N., Marugan, J., Ferrer, M., Bonifacino, J. S. and DePamphilis, M. L. (2019). A family of PIKFYVE inhibitors with therapeutic potential against autophagy-dependent cancer cells disrupt multiple events in lysosome homeostasis. Autophagy 15, 1694-1718.

Shisheva, A. (2008). PIKfyve: Partners, significance, debates and paradoxes. Cell biology international 32, 591-604.

Silver, K. E. and Harrison, R. E. (2011). Kinesin 5B Is Necessary for Delivery of Membrane and Receptors during Fc R-Mediated Phagocytosis. The Journal of Immunology 186, 816825 .

Sim Choi, H., Woo Kim, J., Cha, Y. and Kim, C. (2006). A Quantitative Nitroblue Tetrazolium Assay for Determining Intracellular Superoxide Anion Production in Phagocytic Cells. Journal of Immunoassay and Immunochemistry 27, 31-44.

Song, S., Cong, W., Zhou, S., Shi, Y., Dai, W., Zhang, H., Wang, X., He, B. and Zhang, Q. (2019). Small GTPases: Structure, biological function and its interaction with nanoparticles. Asian Journal of Pharmaceutical Sciences 14, 30-39.

Sridhar, S., Patel, B., Aphkhazava, D., Macian, F., Santambrogio, L., Shields, D. and Cuervo, A. M. (2013). The lipid kinase PI4KIII $\beta$ preserves lysosomal identity. EMBO 
Journal 32, 324-339.

Stenmark, H., Aasland, R. and Driscoll, P. C. (2002). The phosphatidylinositol 3-phosphatebinding FYVE finger. FEBS Letters 513, 77-84.

Storrie, B. and Desjardins, M. (1996). The biogenesis of lysosomes: Is it a kiss and run, continuous fusion and fission process? BioEssays 18, 895-903.

Sun, S.-Y. (2010). N-acetylcysteine, reactive oxygen species and beyond. Cancer biology \& therapy $\mathbf{9}, 109-10$.

Tamariz, E. and Varela-Echavarrĩ-a, A. (2015). The discovery of the growth cone and its influence on the study of axon guidance. Frontiers in Neuroanatomy $\mathbf{9}, 51$.

Taulet, N., Delorme-Walker, V. D. and Line Dermardirossian, C. (2012). Reactive Oxygen Species Regulate Protrusion Efficiency by Controlling Actin Dynamics.

Thormä, M., Marx, A., Sack, S. and Mandelkow, E. (1998). The Coiled-Coil Helix in the Neck of Kinesin.

Ti, S.-C., Alushin, G. M. and Kapoor, T. M. (2018). Human $\beta$-Tubulin Isotypes Can Regulate Microtubule Protofilament Number and Stability. Developmental Cell 47, 175-190.e5.

Tovey, C. A. and Conduit, P. T. (2018). Microtubule nucleation by $\gamma$-tubulin complexes and beyond. Essays in biochemistry 62, 765-780.

Traynor-Kaplan, A., Kruse, M., Dickson, E. J., Dai, G., Vivas, O., Yu, H., Whittington, D. and Hille, B. (2017). Fatty-acyl chain profiles of cellular phosphoinositides. Biochimica et biophysica acta. Molecular and cell biology of lipids 1862, 513-522.

Tsuruta, F., Green, E. M., Rousset, M. and Dolmetsch, R. E. (2009). PIKfyve regulates CaV1.2 degradation and prevents excitotoxic cell death. The Journal of cell biology 187, $279-94$.

Turrens, J. F. (2003). Mitochondrial formation of reactive oxygen species. The Journal of physiology 552, 335-44.

Van Der Kant, R., Jonker, C. T. H., Wijdeven, R. H., Bakker, J., Janssen, L., Klumperman, J. and Neefjes, J. (2015). Characterization of the Mammalian CORVET 
and HOPS Complexes and Their Modular Restructuring for Endosome Specificity*.

Vega-Rubin-de-Celis, S., Peña-Llopis, S., Konda, M. and Brugarolas, J. (2017). Multistep regulation of TFEB by MTORC1. Autophagy 13, 464.

Vonderheit, A. and Helenius, A. (2005). Rab7 Associates with Early Endosomes to Mediate Sorting and Transport of Semliki Forest Virus to Late Endosomes. PLoS Biology 3, e233.

Wada, Y., Cardinale, I., Khatcherian, A., Chu, J., Kantor, A. B., Gottlieb, A. B., Tatsuta, N., Jacobson, E., Barsoum, J. and Krueger, J. G. (2012). Apilimod inhibits the production of IL-12 and IL-23 and reduces dendritic cell infiltration in psoriasis. PloS one 7, e35069.

Wang, T. and Hong, W. (2006). RILP interacts with VPS22 and VPS36 of ESCRT-II and regulates their membrane recruitment. Biochemical and biophysical research communications 350, 413-23.

Wang, W., Gao, Q., Yang, M., Zhang, X., Yu, L., Lawas, M., Li, X., Bryant-Genevier, M., Southall, N. T., Marugan, J., et al. (2015a). Up-regulation of lysosomal TRPML1 channels is essential for lysosomal adaptation to nutrient starvation. Proceedings of the National Academy of Sciences of the United States of America 112, E1373-81.

Wang, W., Cao, L., Wang, C., Gigant, B. and Knossow, M. (2015b). Kinesin, 30 years later: Recent insights from structural studies. Protein science : a publication of the Protein Society 24, 1047-56.

Weaver, B. A. (2014). How Taxol/paclitaxel kills cancer cells. Molecular biology of the cell 25, 2677-81.

Wennerberg, K., Rossman, K. L. and Der, C. J. (2005). The Ras superfamily at a glance. Journal of cell science 118, 843-6.

Wijdeven, R. H., Janssen, H., Nahidiazar, L., Janssen, L., Jalink, K., Berlin, I. and Neefjes, J. (2016). Cholesterol and ORP1L-mediated ER contact sites control autophagosome transport and fusion with the endocytic pathway. Nature Communications 7, 11808.

Wilson, C. and González-Billault, C. (2015). Regulation of cytoskeletal dynamics by redox 
signaling and oxidative stress: implications for neuronal development and trafficking. Frontiers in cellular neuroscience $\mathbf{9}, 381$.

Xiao, Q., Hu, X., Wei, Z. and Tam, K. Y. (2016). Cytoskeleton Molecular Motors: Structures and Their Functions in Neuron. International Journal of Biological Sciences 12, 10831092.

Yamazaki, T., Kawai, C., Yamauchi, A. and Kuribayashi, F. (2011). A highly sensitive chemiluminescence assay for superoxide detection and chronic granulomatous disease diagnosis. Tropical medicine and health 39, 41-5.

Yogev, S., Cooper, R., Fetter, R., Horowitz, M. and Shen, K. (2016). Microtubule Organization Determines Axonal Transport Dynamics. Neuron 92, 449-460.

Yu, L., McPhee, C. K., Zheng, L., Mardones, G. A., Rong, Y., Peng, J., Mi, N., Zhao, Y., Liu, Z., Wan, F., et al. (2010). Termination of autophagy and reformation of lysosomes regulated by mTOR. Nature 465, 942-6.

Yu, L., Chen, Y. and Tooze, S. A. (2018). Autophagy pathway: Cellular and molecular mechanisms. Autophagy 14, 207-215.

Zeigerer, A., Gilleron, J., Bogorad, R. L., Marsico, G., Nonaka, H., Seifert, S., EpsteinBarash, H., Kuchimanchi, S., Peng, C. G., Ruda, V. M., et al. (2012). Rab5 is necessary for the biogenesis of the endolysosomal system in vivo. Nature 485, 465-470.

Zhai, Y., Kronebusch, P. J., Simon, P. M. and Borisy, G. G. (1996). Microtubule Dynamics at the G2/M Transition: Abrupt Breakdown of Cytoplasmic Microtubules at Nuclear Envelope Breakdown and Implications for Spindle Morphogenesis. The Journal of Cell Biology 135,.

Zhang, X., Cheng, X., Yu, L., Yang, J., Calvo, R., Patnaik, S., Hu, X., Gao, Q., Yang, M., Lawas, M., et al. (2016). MCOLN1 is a ROS sensor in lysosomes that regulates autophagy. Nature communications 7, 12109.

Zhang, H., Huang, T., Hong, Y., Yang, W., Zhang, X., Luo, H., Xu, H. and Wang, X. (2018). The Retromer Complex and Sorting Nexins in Neurodegenerative Diseases. Frontiers in aging neuroscience 10, 79. 
Zhao, G., Cao, K., Xu, C., Sun, A., Lu, W., Zheng, Y., Li, H., Hong, G., Wu, B., Qiu, Q., et al. (2017). Crosstalk between Mitochondrial Fission and Oxidative Stress in ParaquatInduced Apoptosis in Mouse Alveolar Type II Cells. International journal of biological sciences 13, 888-900.

Zheng, K., Li, Y., Wang, S., Wang, X., Liao, C., Hu, X., Fan, L., Kang, Q., Zeng, Y., Wu, X., et al. (2016). Inhibition of autophagosome-lysosome fusion by ginsenoside Ro via the ESR2-NCF1-ROS pathway sensitizes esophageal cancer cells to 5-fluorouracil-induced cell death via the CHEK1-mediated DNA damage checkpoint. Autophagy 12, 1593-1613.

Zigmond, S. H. (2000). How WASP regulates actin polymerization. The Journal of cell biology 150, F117-20.

Zolov, S. N., Bridges, D., Zhang, Y., Lee, W.-W., Riehle, E., Verma, R., Lenk, G. M., Converso-Baran, K., Weide, T., Albin, R. L., et al. (2012). In vivo, Pikfyve generates $\mathrm{PI}(3,5) \mathrm{P} 2$, which serves as both a signaling lipid and the major precursor for PI5P. Proceedings of the National Academy of Sciences of the United States of America 109, 17472-7.

Zoncu, R., Perera, R. M., Balkin, D. M., Pirruccello, M., Toomre, D. and De Camilli, P. (2009). A phosphoinositide switch controls the maturation and signaling properties of APPL endosomes. Cell 136, 1110-21.

Zou, J., Hu, B., Arpag, S., Yan, Q., Hamilton, A., Zeng, Y.-S., Vanoye, C. G. and Li, J. (2015). Reactivation of Lysosomal Ca2+ Efflux Rescues Abnormal Lysosomal Storage in FIG4-Deficient Cells. Journal of Neuroscience 35, 6801-6812. 